\title{
On-Line Monitoring and Diagnostics of the Integrity of Nuclear Plant Steam Generators and Heat Exchangers
}

\author{
Final Report
}

Report No. DE-FG07-01ID14114/UTNE-08

NEER Grant Number: DE-FG07-01ID14114

\section{Volume 2}

Structural Defect Monitoring and Diagnosis Using Analysis of Transient Guided Acoustic Signals

Belle R. Upadhyaya

J. Wesley Hines

(Principal Investigators)

Baofu Lu

The University of Tennessee Nuclear Engineering Department 209 Pasqua Engineering Building

Knoxville, TN 37996-2300

E-mail: bupadhya@utk.edu

DOE Program Manager for NEER Idaho Operations Office

Nancy A. Elizondo

September 2004

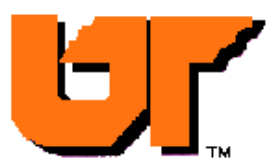




\section{ACKNOWLEDGMENTS}

The research was supported by a U.S. Department of Energy Nuclear Engineering Education Research (NEER) grant with the University of Tennessee, Knoxville (grant number DE-FG07-01ID14114). We want to acknowledge the assistance provided by the NEER grant office, DOE Idaho Operations. The authors wish to thank Professor Emeritus Rafael Perez, University of Tennessee, for the discussion of Hilbert-Huang transformation and its implementation. The authors greatly appreciate the assistance provided by Richard Bailey and Gary Graves of the Nuclear Engineering Department in developing the experimental facilities for this project.

\section{DISCLAIMER}

Any opinions, findings, and conclusions or recommendations expressed in this report are those of the authors and do not necessarily reflect the views of the Department of Energy. 


\section{SUMMARY}

Integrity monitoring and flaw diagnostics of flat beams and tubular structures was investigated in this research task using guided acoustic signals. A piezo-sensor suite was deployed to activate and collect Lamb wave signals that propagate along metallic specimens. The dispersion curves of Lamb waves along plate and tubular structures are generated through numerical analysis. Several advanced techniques were explored to extract representative features from acoustic time series. Among them, the HilbertHuang transform (HHT) is a recently developed technique for the analysis of non-linear and transient signals. A moving window method was introduced to generate the local peak characters from acoustic time series, and a zooming window technique was developed to localize the structural flaws.

The time-frequency analysis and pattern recognition techniques were combined for classifying structural defects in brass tubes. Several types of flaws in brass tubes were tested, both in the air and in water. The techniques also proved to be effective under background/process noise. A detailed theoretical analysis of Lamb wave propagation was performed and simulations were carried out using the finite element software system ABAQUS. This analytical study confirmed the behavior of the acoustic signals acquired from the experimental studies.

The report presents the background the analysis of acoustic signals acquired from piezo-electric transducers for structural defect monitoring. A comparison of the use of time-frequency techniques, including the Hilbert-Huang transform, is presented. The report presents the theoretical study of Lamb wave propagation in flat beams and tubular structures, and the need for mode separation in order to effectively perform defect diagnosis. The results of an extensive experimental study of detection, location, and isolation of structural defects in flat aluminum beams and brass tubes are presented.

The results of this research show the feasibility of on-line monitoring of small structural flaws by the use of transient and nonlinear acoustic signal analysis, and its implementation by the proper design of a piezo-electric transducer suite. 


\section{CONTENTS}

SUMMARY

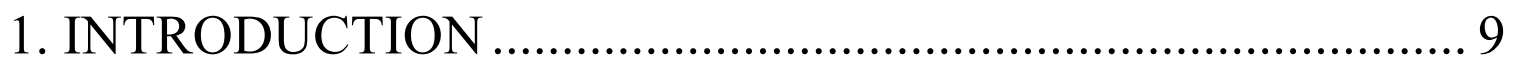

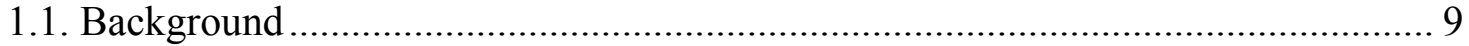

1.2. Review of the Applications of Guided Acoustics............................................... 10

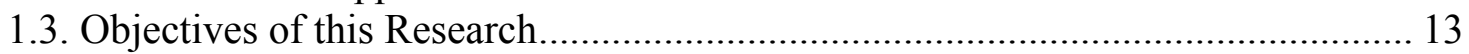

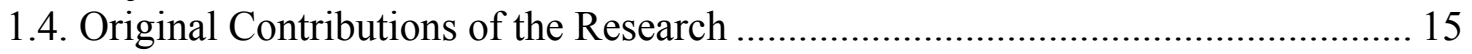

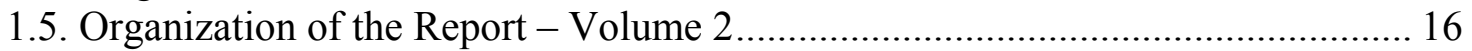

2. EXPERIMENTAL RESEARCH ....................................... 19

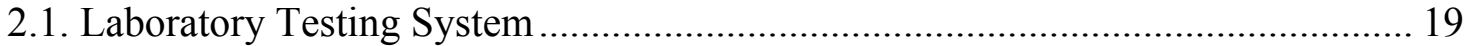

2.2. Piezo-electric Materials and Piezo-sensors............................................................ 20

2.3. Activation of Guided Acoustics Using Piezo-sensors ............................................ 24

3. FUNDAMENTALS OF LAMB WAVE THEORY ............... 27

3.1. Elastic Wave Propagation Along Thin Plates................................................. 27

3.2. Elastic Waves in Metal Tubes....................................................................... 34

3.3. Elastic Waves in Metal Structures Submerged in Water ................................... 50

3.3.1. Plate specimen immersed in water............................................................... 50

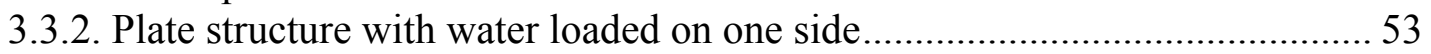

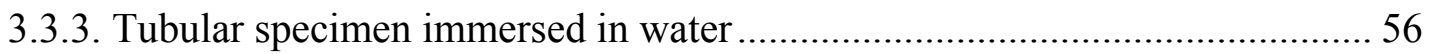

3.3.4. Tubular structure with water in contact on the outside.................................. 58

4. DIGITAL SIGNAL PROCESSING (DSP) TECHNIQUES FOR NON-STATIONARY ACOUSTIC DATA ................................ 60

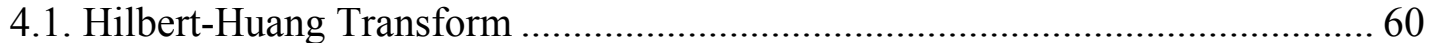

4.2. Moving Window Method for the Analysis of Time Series of Lamb .................. 63

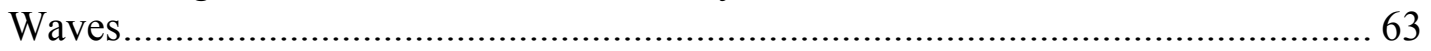

4.3. Window Zooming Method for the Analysis of Lamb Wave Data...................... 66

4.4. Wavelet Transformation and Eigen-face Analysis ........................................... 67

4.5. Comparison of Wavelet Transform with HHT ...................................................69 69

5. MODE SEPARATION OF LAMB WAVES …….................... 78

6. STRUCTURAL DIAGNOSTICS OF ALUMINUM PLATES 82

6.1. Flaw Detection and Localization Using HHT ………..................................... 83

6.2. Flaw Detection and Localization Using Extrema Extraction ............................. 92

6.3. Selection of the Resonant Frequency for Aluminum Plate................................. 95

7. INTEGRITY MONITORING OF METAL TUBING ............ 97

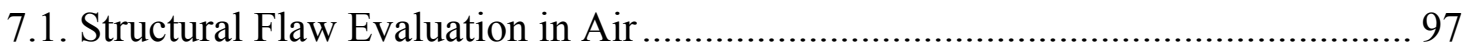

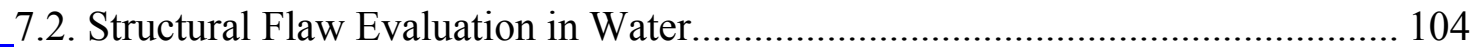

7.3. Comparison of Structural Flaw Evaluation in Air and in Water ......................... 108

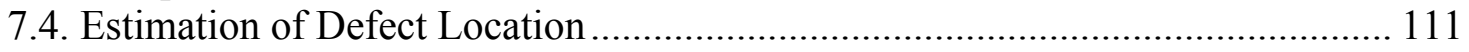

7.4.1. Flaw localization for brass tube in air........................................................ 111

7.4.2 Flaw Localization for Brass Tubes in Water Through Zooming Windows.. 114

7.5. Noise Reduction of Acoustic Signals in Brass Tubes........................................ 117

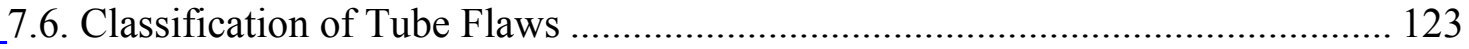


7.7. Summary of Tubular Structural Evaluation

8. SIMULATION OF LAMB WAVE PROPAGATION USING

THE FINITE ELEMENT CODE ABAQUS ........................... 130

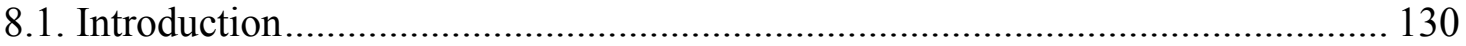

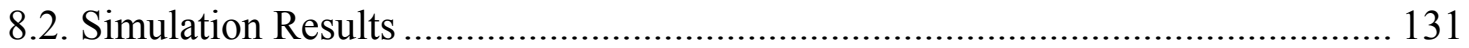

8.3. Concluding Remarks on Simulation Using ABAQUS ......................................... 139

9. CONCLUSIONS AND SUGGESTIONS FOR FUTURE

WORK

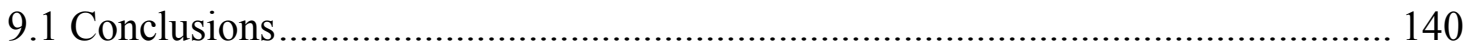

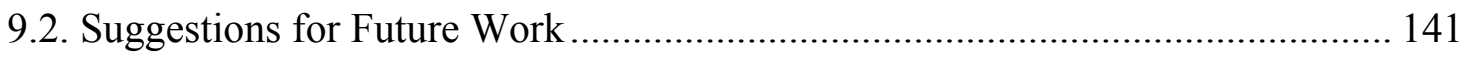

BIBLIOGRAPHY ...................................................... 143

APPENDICES ............................................................... 150

Appendix A: Cylindrical Coordinate Used in Tube Analysis .................................. 150

Appendix B: More moving window results for brass tubes in air ............................. 152

Appendix C: MATLAB Code Lamb wave Numerical Solution ............................... 156

Appendix D: MATLAB Code for HHT........................................................... $165 \underline{5}$

Appendix E: MATLAB Code for Moving Window Algorithm.............................. 1699

Appendix F: LabVIEW Interface for Lamb Wave Experiments............................. $174 \underline{4}$ 


\section{List of tables}

Table 7.1. Five structural conditions tested for a brass tube ( 3 feet long) in the air........98

Table 7.2. Five conditions tested for a brass tube (2 feet long) in water (\#10) ............. 105

Table 7.3. Six conditions tested for a brass tube in both air and water (\#4) .................. 108

\section{List of Figures}

Figure 2.1. Experimental modules for interrogation of typical specimens..................... 20

Figure 2.2. Experimental setup for testing brass tubing in water. ................................ 20

Figure 2.5. Experimental specimens with sensor and structural flaw. (a) Brass tube; (b)

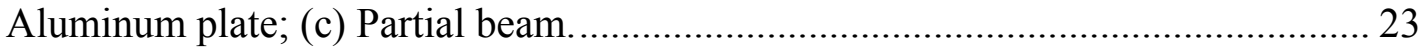

Figure 2.6. Methods for Lamb wave generation........................................................ 24

Figure 3.1. Guided acoustic waves in a plate-like structure. ...................................... 27

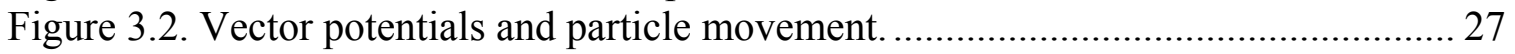

Figure 3.3. Dispersion curves for a traction free aluminum beam............................... 32

Figure 3.4. Group speed of Lamb wave in a traction free aluminum beam. .................. 33

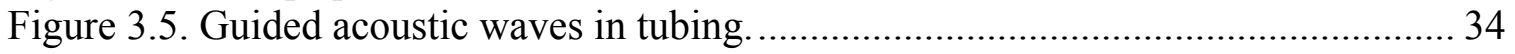

Figure 3.6. Lamb wave modes in tubular structures. ................................................ 36

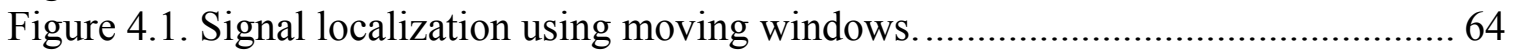

Figure 4.2. Signal localized properties using zooming windows. ................................ 66

Figure 4.3. Energy distribution of wavelet transformation in the time-frequency domain.

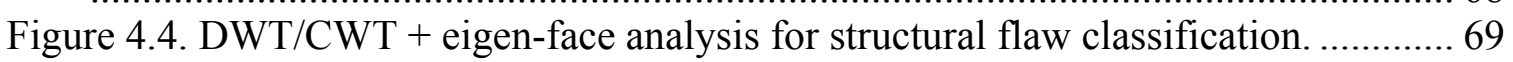

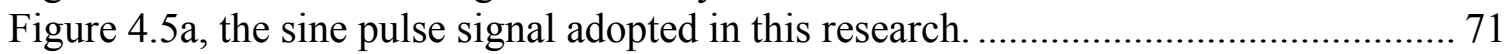

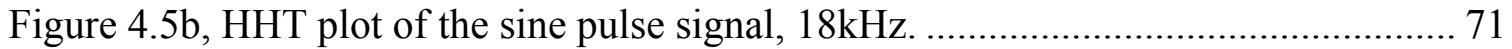

Figure 4.5c, WT plot of the sine pulse signal, Morlet wavelet is used. The leakage of the

energy is obvious compared with $7.5 \mathrm{~b}$............................................................ 72

Figure 4.5d, WT + Hilbert transform can improve the concentration of instant frequency.

Figure $4.6 \mathrm{~b}$, HHT of the amplitude modulated signals illustrates frequency modulation

introduced by amplitude modulation is small................................................... 74

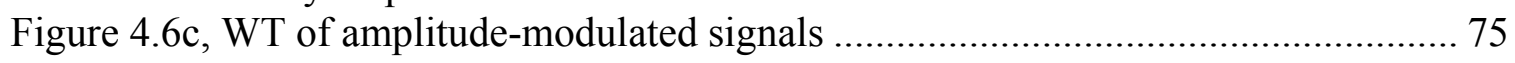

Figure 4.6d, WT + Hilbert transform for amplitude modulated signals. ....................... 75

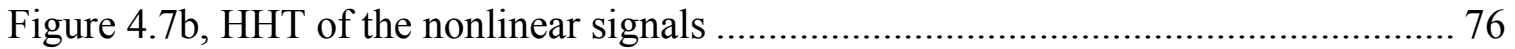

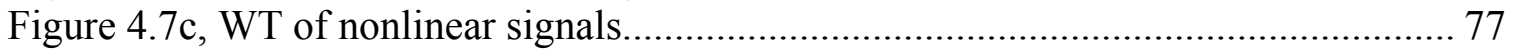


Figure 4.7d, WT + Hilbert transform for nonlinear signals.................................... 77

Figure 5.1. Raw signals from brass tube................................................................... 79

Figure 5.2. Separated symmetric and anti-symmetric mode signals. ........................... 80

Figure 6.5. Time-frequency representation of HHT of Lamb wave signal in a normal

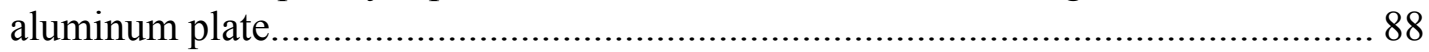

Figure 6.6. Time-frequency representation of HHT of Lamb wave signal in an aluminum

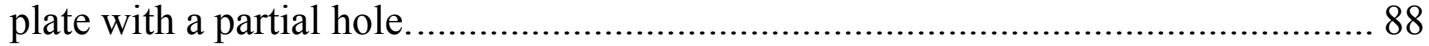

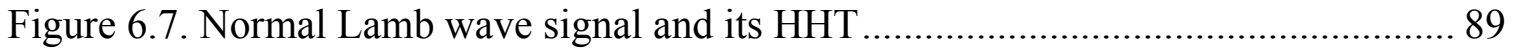

Figure 6.8. Lamb wave signal from an aluminum beam with two clips located near the

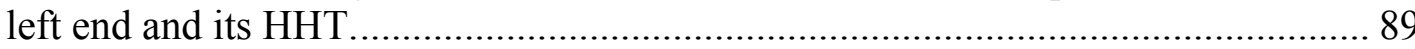

Figure 6.9. Lamb wave signal from an aluminum beam with two clips located near the

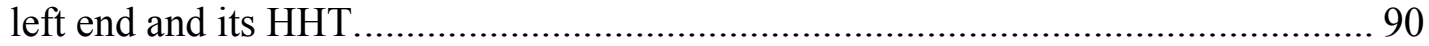

Figure 6.10. Lamb wave signal from an aluminum beam with two clips located in the

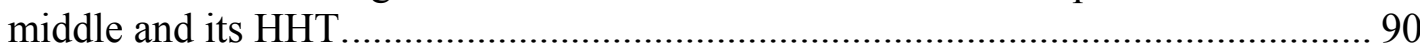

Figure 6.11. Lamb wave signal from an aluminum beam with two clips located near the

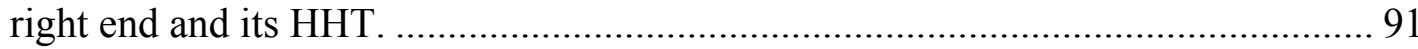

Figure 6.12. Lamb wave signal from an aluminum beam with two clips located near the

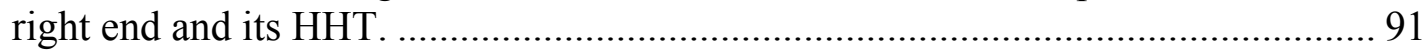

Figure 6.13. Lamb waves and envelope extraction............................................... 93

Figure 6.14. Passive Lamb wave signals for aluminum beam under different conditions.

Figure 6.15. The signals between the first and the second peaks for different flaw types.

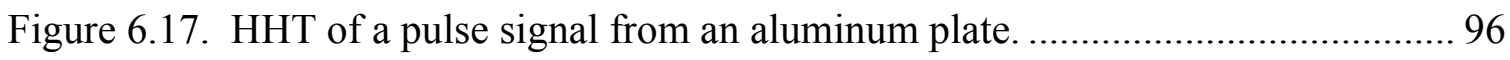

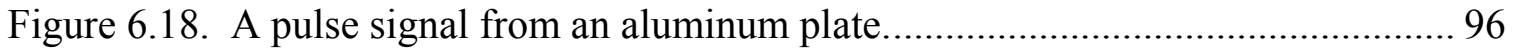

Figure 7.1. Amplitude change of local peaks of anti-symmetric mode signals, propagating from the right to the left, with $14 \mathrm{kHz}$ input frequency....................... 98

Figure 7.2. Change of variance of local peaks from anti-symmetric mode signals propagating from the right to the left end, with input frequency $14 \mathrm{kHz}$................ 99

Figure 7.3. Change of left part weight center of local peaks from anti-symmetric mode signals propagating from the right to the left end, input frequency $14 \mathrm{kHz}$............ 99

Figure 7.4. Change of right part weight center of local peaks from anti-symmetric mode signals propagating from the right to the left end, with input frequency $14 \mathrm{kHz} . . .100$

Figure 7.5. Amplitude change of local peaks of symmetric mode signals propagating

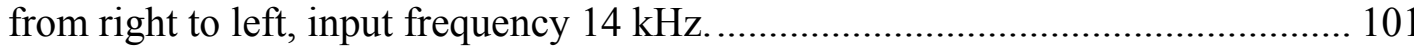

Figure 7.6. Change of variance of local peaks of symmetric mode signals, propagating from right to left, input frequency $14 \mathrm{kHz}$.

Figure 7.7. Change of left part weight center of local peaks of symmetric mode signals

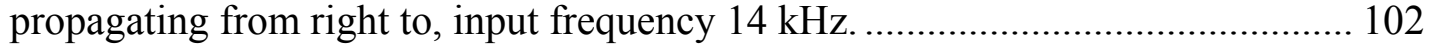

Figure 7.8. Change of right part weight center of local peaks of symmetric mode signals, propagating from right to left, input frequency $14 \mathrm{kHz}$.....

Figure 7.9. Amplitude change of local peaks of anti-symmetric mode signals in the water, propagating from left to right, with $13 \mathrm{kHz}$ input frequency...................... 106

Figure 7.10. Variance change of local peaks of anti-symmetric mode signals in the water, propagating from left to right, with $13 \mathrm{kHz}$ input frequency............................. 106 
Figure 7.11. Left weight center change of local peaks of anti-symmetric mode signals in the water, propagating from left to right, with $13 \mathrm{kHz}$ input frequency.

Figure 7.12. Right weight center change of local peaks of anti-symmetric mode signals in the water, propagating from left to right, with $13 \mathrm{kHz}$ input frequency...

Figure 7.13. Amplitude change of local peaks from anti-symmetric mode signals, propagating from the left to the right, with input frequency $16 \mathrm{kHz}$.

Figure 7.14. Change of variance of local peaks from anti-symmetric mode signals, propagating from the left to the right, with input frequency $16 \mathrm{kHz}$.

Figure 7.15. The change of left part weight center of local peaks from anti-symmetric mode signals, propagating from the left to the right, with input frequency $16 \mathrm{kHz}$.

Figure 7.16. The change of right part weight center of local peaks from anti-symmetric mode signals, propagating from the left to the right, with input frequency $16 \mathrm{kHz}$.

Figure 7.17. The zooming windows and the diverging points.

Figure 7.18. The change of right part weight center of the first local peak as the zooming window changing size from 100 to 1200 , using anti-symmetric mode signals, propagating from the right to the left, input frequency $13 \mathrm{kHz}$.

Figure 7.19. The change of left part weight center of the second local peak as the zooming window changing size from 100 to 1200, using anti-symmetric mode signals, propagating from the right to the left, input frequency $13 \mathrm{kHz}$

Figure 7.20. The change of right part weight center of the first local peak as the zooming window changing size from 100 to 1200 , using anti-symmetric mode signals, propagating from the left to the right, input frequency $13 \mathrm{kHz}$.

Figure 7.21. The change of left part weight center of the second local peak as the zooming window changing size from 100 to 1200 , using anti-symmetric mode signals, propagating from the left to the right, input frequency $13 \mathrm{kHz}$.

Figure 7.22. The change of right part weight center of the first local peak as the zooming window changing size from 100 to 1200 , anti-symmetric mode signals, propagating from the right to the left, input frequency $13 \mathrm{kHz}$.

Figure 7.23. The change of right part weight center of the second local peak as the zooming window changing size from 100 to 1200, anti-symmetric mode signals, propagating from the right to the left, input frequency $13 \mathrm{kHz}$.

Figure 7.24. The change of right part weight center of the first local peak as the zooming window changing size from 100 to 1200 , anti-symmetric mode signals, propagating from the left to the right, input frequency $13 \mathrm{kHz}$.

Figure 7.25. The change of right part weight center of the second local peak as the zooming window changing size from 100 to 1200, anti-symmetric mode signals, propagating from the left to the right, input frequency $13 \mathrm{kHz} \ldots \ldots \ldots \ldots \ldots \ldots \ldots \ldots \ldots . . . .116$

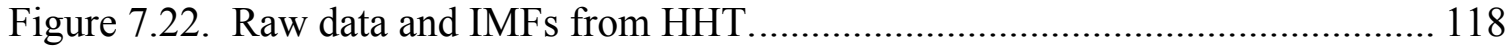

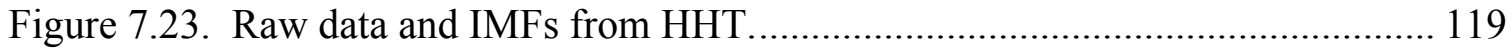

Figure 7.24. Raw data and the reconstructed data. ................................................. 119

Figure 7.25. Amplitude change of local peaks from anti-symmetric mode signal after denoising, with input frequency $13 \mathrm{kHz}$.

Figure 7.26. Spread change of local peaks from anti-symmetric mode signal after denoising, with input frequency $13 \mathrm{kHz}$. 
Figure 7.27. Left gravity centers of local peaks from anti-symmetric mode signal after

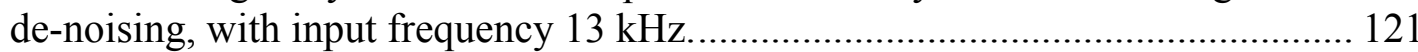

Figure 7.28. Right gravity centers of local peaks from anti-symmetric mode signal after de-noising, with input frequency $13 \mathrm{kHz}$.

Figure 7.29. Spread change of local peaks from anti-symmetric mode signal before denoising, with input frequency $13 \mathrm{kHz}$

Figure 7.30. Left gravity centers of local peaks from anti-symmetric mode signal with noise, with input frequency $13 \mathrm{kHz}$.

Figure 7.31. Right gravity centers of local peaks from anti-symmetric mode signal with

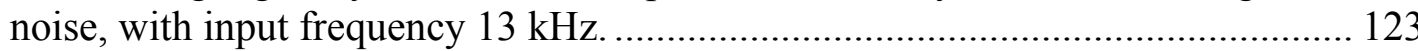

Figure 7.32. The classification of tube defects. ........................................................ 124

Figure 7.33. DWT decomposition of acoustic waves in a brass tube............................ 126

Figure 7.34. The tube defect classification using DWT + Eigen-face............................ 127

Figure 7.36. Lamb wave signals with noise from a brass tube..................................... 129

Figure 8.11. Particle displacement at one point on the plate..................................... 138

Figure 8.12.6. Frequency response for brass tube (from Simulation). ........................ 138

Figure A.1. Amplitude change of local peaks of anti-symmetric mode signals, propagating from left to right, input frequency $14 \mathrm{kHz}$.

Figure A.2. The change in variance of local peaks of anti-symmetric mode signals, propagating from left to right, with $14 \mathrm{kHz}$ input frequency.

Figure A.3. The change in left part weight center of local peaks of anti-symmetric mode signals, propagating from left to right, input frequency $14 \mathrm{kHz}$.

Figure A.4. The change in right part weight center of local peaks of anti-symmetric mode signals, propagating from left to right, input frequency $14 \mathrm{kHz}$.

Figure A.5. Amplitude change in local peaks of anti-symmetric mode signals, propagating from left to right, input frequency $13 \mathrm{kHz}$

Figure A.6. The change in variance of local peaks of anti-symmetric mode signals, propagating from left to right, input frequency $13 \mathrm{kHz}$.

Figure A.7. The change in left part weight center of local peaks of anti-symmetric mode signals, propagating from left to right, input frequency $13 \mathrm{kHz}$.

Figure A.8. The change in right part weight center of local peaks of anti-symmetric mode signals, propagating from left to right, input frequency $13 \mathrm{kHz}$. 


\section{INTRODUCTION}

\subsection{Background}

Nuclear power plant components, such as steam generators (SGs), heat exchangers, pressure vessels, and piping are exposed to high temperature, high pressure,

and high radiation environment. Key components such as SGs and pressure vessels have stringent design requirements regarding their structural integrity. To increase the safety and reliability of a nuclear power plant, the monitoring of the integrity of key equipment is very important. Information about conditions such as tube cracks, corrosion, pitting, and fouling must be available in order to keep severe damages from occurring.

Some nondestructive testing techniques have been developed and implemented for structural defect inspection during manufacture and for routine maintenance process. The well known methods for in-service inspection include eddy current testing, ultrasonic testing, visual inspection, and others. These methods are fairly effective and accurate in detecting structural flaws in SG tubes, pressure vessels, and steam pipes, but these inspections are usually off-line and slow. Hence the traditional nondestructive examination (NDE) methods are not suitable for in-situ and on-line monitoring. On the other hand, nuclear reactor surveillance systems are not capable of providing complete intrinsic information about structures in a reactor systems. Currently there are no techniques that can be used during plant operation to collect internal information and evaluate structural integrity of key components.

An innovative idea was proposed in this research to develop an intelligent system such that guided active acoustic signals could be generated in SG tubing any time during plant operation, and passive signals could be collected with sufficient information to evaluate its integrity. The tested structure would be diagnosed using passive acoustic signals through advanced non-stationary signal processing techniques. Although there is a large body of research performed in Lamb waves and NDE, many problems still exist. These include the selection of input frequency band, sensor deployment, and feature extraction. None have ever studied the change of Lamb wave properties in specimens 
immersed in water and their application in structural health monitoring, which is important in many circumstances and is one of the key problems studied in this research.

In order to perform the detection, localization, and classification of flaws in tubing or plate-like structures, the time frequency analysis methods were explored to extract representative features. Pattern classification techniques were used to categorize structural defects in ether air or in water. In addition, a multi-sensor suite was developed to monitor the wave propagation from multiple perspectives. As a viable technique, wavelet transform (WT) (continuous and discrete WT) which is an effective nonstationary and linear data decomposition technique, was used in this study for bandlimited feature extraction. Another recently developed method, the Hilbert-Huang transform (HHT), provides a more efficient time-frequency analysis of signals from nonlinear systems. The application of HHT for elastic wave analysis was an important step towards accurate structural heath diagnostics in this research. Other transient signal processing techniques, such as the moving window and zooming window, were implemented to deal with localized acoustic signal properties in the time domain.

In addition, to verify the experimental work, simulation of Lamb waves was performed in tubes and plate-like structures. A finite element code, called ABAQUS, was used to simulate Lamb wave propagation.

\subsection{Review of the Applications of Guided Acoustics}

Named after the English scientist Horace Lamb, in honor of his fundamental contributions to wave propagation, Lamb wave has attracted a broad range of studies of its properties and applications. During the 1960s Viktorov [22] elaborated the properties of elastic guided waves (Lamb and Rayleigh waves), the applications in the NDE field, and the methods of activating specified guided waves. His work has become a cornerstone for the many subsequent studies. Auld [3] systematically summarized the wave propagation in elastic media, including non-homogeneous materials such as the piezo- electric materials. The use of Lamb waves for the detection of inclusions was analyzed theoretically in his work. Recently, Rose [31] studied the practical applications of guided waves from experimental and theoretical perspectives. The wave propagation in multi-layer materials was discussed and the utilization of mode change due to the 
phenomenon of scattering was studied to determine the most sensitive input frequency and the appropriate mode. The idea of utilizing some specific modes of guided waves was introduced in his work.

Many other researchers are also involved in the study of Lamb waves, including practical applications. Among them Ditri [29] used S-parameter formalism to study the phenomenon of scattering of Lamb waves from a circumferential crack in an isotropic hollow cylinder. Similarly McKeon [23] explored higher order plate theory to derive analytical solutions for the scattering of the lowest order symmetric Lamb waves from a circular inclusion in plate like structures. The results were used to explain the scattering effects found in Lamb wave tomography. Alleyne [11] (1998) studied the reflection of $\mathrm{L}(0,2)$ mode Lamb wave from notches in pipe-like structures, and the relationship between reflection ratio and the depth of notch. The pulse-echo method was adopted in his research. However, the experimental setup is not suitable for in situ and on-line structural inspection because of its complexity.

In 2000, Malyarenko et al. [16] described the application of Lamb wave tomography for mapping the flaws in multi-layer aircraft materials. A circular array of space transducers was set up for the reconstruction of tomography, which was used to judge the health of aircraft structures. The study was aimed at scanning a large area quickly and automatically. Although that technique cannot be applied to tube like structures, it is still an important step towards the application of Lamb wave techniques in the aerospace industry. Another important work was reported by Motegi [32] in 1999 about Lamb wave propagation in water-immersed inhomogeneous plates. The radiation of Lamb waves into water from the specimen was analyzed. This work is mentioned here because of the importance of the interaction between water and immersed specimens in this study.

In 2001, Halabe and Franklin [58] tried to detect fatigue cracks in metallic members using the statistical properties of guided waves in the frequency domain. The Rayleigh waves were produced and several types of crack-like defects (for example, micro fatigue, macro fatigue) were tested using five-cycle sine pulse excitation with 2.25 $\mathrm{MHz}$ of central frequency. The study illustrates the sensitivity of Rayleigh waves to surface flaws, but location and classification were not studied in their research. In 2001 
Jung [62] detected discontinuities in concrete structures using Lamb waves and frequency domain analysis.

Time-frequency analysis methods are of importance for characterizing acoustic waves. Niethammer and Jacobs [40] compared four methods of time-frequency representations of Lamb waves. The reassigned spectrogram (from short-time Fourier transform (STFT)), the reassigned scalogram (from wavelet transform (WT)), WignerVille distribution (WVD) and Hilbert transform were used to represent multi-mode Lamb waves. The advantages and shortcomings were discussed. The results showed that spectrogram and smoothed WVD gave the best time-frequency distribution for wide-band Lamb waves.

In 2002, Valle et al. [7] performed the study of flaw localization with reassigned spectrogram of detected Lamb modes using a modified signal processing technique. The spectrogram was generated by STFT, and the image change due to the flaw reflection was used to locate notch-type flaws. Only one type of flaw was studied and the accuracy of the detection depended heavily on the signal quality; a high level of noise would provide a big challenge in the performance of the algorithm. Although the scope of this research is limited, it embodies some good ideas such as using non-contact methods to generate guided waves, and utilizing advanced signal processing techniques to explore the hidden information. Similarly, in the work of Clezio [15], the interaction between cracks and the first symmetric Lamb mode $S_{0}$ in an aluminum plate placed in a vacuum were demonstrated using both experiments and finite element simulations. The work illustrates a nonlinear relationship between crack thickness, and reflection and transmission coefficients. Another type of flaw, a hole in an aluminum plate, was studied by Fromme and Sayir [47] in 2002. The active Lamb wave was selectively excited to have an anti-symmetrical mode using piezoelectric transducers, and is currently a very popular method for Lamb wave activation. The scattering coefficient is calculated using Mindlin's theory and a classical plate theory.

The application of Lamb waves for flaw detection in composite structures has also attracted many researchers. In 2002, Kessler [54] studied health monitoring of composite materials, either in plate or tubular structure. The properties of Lamb wave propagation in composite structures were studied using ABAQUS, a finite element simulation code, 
and experiments using piezo-transducers. The experimental data were processed using continuous wavelet transform to increase the sensitivity of flaw detection. Similarly Yuan [68] intended to establish an on-line damage detection algorithm for composite structures where time and frequency information were used for integrity evaluation and wavelet analysis was used to reduce raw data noise. Another researcher, Paget [4], performed the damage assessment in composites through Lamb waves using adaptive wavelet decomposition technique that was sensitive to small damages.

There are also many other studies about the application of guided waves. For example, Kawiecki and Seagle [79] detected the damages in aluminum plates and concrete blocks through shifts in frequency resonance peaks of Lamb waves.

However, most of these works focused on the detection of structural flaws in either single or composite materials, none was able to classify flaw type and locate the flaw simultaneously. In contrast, the research reported here focused on developing an on-line and in-situ structural flaw classification approach through smart signal collection and analysis. In order to locate and classify structural flaws, which are difficult using raw Lamb wave signals, an advanced post signal processing technique was absolutely necessary. As mentioned above, several time-frequency analysis methods have been used to process Lamb wave signals. However, all of these methods, from STFT to wavelet analysis, use linear transformations. A new nonlinear and non-stationary signal processing technique, called the Hilbert-Huang transform (HHT), was introduced in this research project, and other advanced DSP techniques were developed for the structural diagnostics of power plant components.

\subsection{Objectives of this Research}

Inspired by the idea of guided elastic waves for structural flaw detection, the research reported here was aimed at developing on-line, in-situ structural monitoring techniques for steam generator and heat exchanger tubing to minimize the limitations of data accessibility during plant operation. The new monitoring system would combine the functions of acoustic signal generation, data collection, flaw detection, evaluation, 
isolation and classification. The new techniques could be extended to pressure vessels, steam pipes, and other important equipment to improve the safety of nuclear power plants.

For the most commonly used ultrasonic NDE technique, the equipment adopted is not suitable for on-line testing of steam generator tubes. The post signal processing technique is not mature enough to extract hidden features from complex signals. This research was aimed at studying the feasibility of implementing embedded sensor suites in structural members. The system must be sensitive to small structural changes, and robust under noisy environment. Guided waves, either Lamb waves or Rayleigh waves, would be analyzed since both of them are highly sensitive to structural anomalies. Because of the small tube wall thickness of steam generator tubing, we are interested in the Lamb wave propagation in this study. The application of lamb waves for structural flaw detection is not a new idea. However, there are several practical problems, especially in the extraction of representative features.

In distinction from the previous work (details in Literature review) on Lamb waves for structural defect analysis, this research introduced and implemented some advanced non-stationary signal processing techniques, so that the multi-mode Lamb wave time series could be processed to reveal representative features. Both time and frequency information would be extracted to detect and isolate potential structural flaws, which may cause severe damages with time. Following is a list of significant results of this research.

- A successful design of a smart signal activation and collection system. A multisensor piezo-sensor system is proposed to interrogate test specimens from different directions, and the passive sensors collecting optimal system information from multiple perspectives. Several types of input signals were used in order to determine the optimal frequency band, input signal length, and shapes.

- Comprehensive study of Lamb wave propagation in different media and structures, especially in tubing specimens immersed in water. The aluminum plate was also studied as a benchmark specimen in this research.

- Signal characterization for various flaws. Typical flaws include half depth holes, through hole, notches, and other simulated flaws using clip-on weights. The 
severity of flaw is adjusted through the change of the flaw size such as notch depth and diameter of the hole. Flaw position information was collected to study the localization of unknown structural flaws.

- Structural health evaluation through the change of signal energy, local peak position, extent of spread, etc. The analysis was performed in both time and frequency domains using non-stationary signal decomposition. The sensitivity and robustness of the techniques were evaluated.

- Estimation of the location of structural flaws. It was found that the reflected and diffracted waves could be used for the localization of structural defects using advanced signal processing techniques.

- Defect classification in laboratory specimens, especially brass tubes. Cross correlation of wavelet analysis and Hilbert-Huang transform are being used to extract the signatures for each type of structural defect. Advanced pattern recognition techniques, such as PCA of residual space, was applied to identify flaw type.

- Theoretical study of acoustic wave propagation by simulation of Lamb wave propagation along aluminum plates and brass tubes using the software ABAQUS.

\subsection{Original Contributions of the Research}

In order to realize the on-line and in-situ structural health monitoring for a complex system such as a steam generator, many technical problems need to be tackled including experimental design, data acquisition, data transmission, signal feature extraction, and pattern classification. The original contributions of this research include the following:

i. Development of a smart structural activation and signal collection system that is suitable for Lamb wave separation. A special set-up with multiple piezo-sensors is proposed to generate the mixtures of symmetric and anti-symmetric Lamb waves for both plate and tubular structures. 
ii. Lamb wave mode separation. In this research, mode separation was proved to be very important for tubular structures in order to characterize the flaws, while it is not necessary for aluminum beams because the anti-symmetric wave has much higher energy than the symmetric wave.

iii. New implementation of Hilbert-Huang transform (HHT) for evaluation of various flaws in an aluminum plate. The HHT is more sensitive in detecting to structural flaws than other signal processing methods.

iv. Development of a new moving window method for the extraction of localized features from Lamb waves in tubing. The sensitivity and the robustness of this algorithm will be evaluated quantitatively.

v. Development of a window zooming technique for estimation of the location of the structural flaw in tubing. Diverging points were defined to reflect the distance between the receiving sensors and the defect.

vi. Defect classification using time-frequency analysis and pattern recognition methods.

vii. Noise reduction using HHT decomposition and reconstruction.

viii. Theoretical study of Lamb wave propagation along aluminum plates and brass tubes using the finite-element code ABAQUS. Defect conditions such as partial and through holes would be simulated and compared to experimental results.

\subsection{Organization of the Report - Volume 2}

Section 2 describes the experimental setup developed in this research. The sensor arrangement is demonstrated for the structural test. It then briefly describes the principles of piezoelectric phenomena and piezoelectric materials. The dimension and materials of the piezo-sensors used in this research are then detailed. Finally, this section discusses the exisiting methods for the Lamb wave generation, and their advantages and shortcomings.

The Lamb wave propagation equations are elaborated in Section 3. Lamb waves are described in Cartesian and cylindrical coordinates respectively for aluminum plate and brass tubes. Characteristic equations are derived with appropriate boundary 
conditions. The numerical solutions are then generated and dispersion curves are plotted to demonstrate the change of Lamb wave speed due to the change of Lamb wave mode and input frequency. In the final part of this section, characteristic equations for the structures submerged in water are derived. The continuous stress components and particle displacement on the boundaries are used in system equations.

Section 4 discusses non-stationary signal processing techniques. Hilbert-Huang and wavelet transforms are compared to reveal the differences between linear and nonlinear transformation. Moving window and zooming window techniques are introduced to perform feature extraction from Lamb wave signals propagating along the experimental specimens. Eigen-face analysis was used to classify the structural flaw based on the time-frequency plot of the Wavelet Transform.

In Section 5, the mode separation concept is introduced. The mode separation process takes advantage of the special arrangement of sensor placement. Separated symmetric and anti-symmetric signals were used to verify the wave speed along the brass tube used in the research.

Section 6 describes the monitoring of the aluminum plate structure based on the passive Lamb waves propagating in the test specimen. HHT is utilized in the analysis of the structural resonant frequency, flaw indication, and localization. Another method, called the extrema envelope, was also applied to flaw detection, especially the localization of structural flaws in the plate-like structures.

The focus of Section 7 is on tubular structures. The moving window technique was utilized to extract representative features from the separated anti-symmetric Lamb wave signals such that the health conditions of the tested structure can be evaluated. The features can be also used for flaw classification. Due to the wave reflection, the flaw localization needs the help of a new method called zooming windows. The position of flaw is then approximately decided. The flaw type classification is realized using wavelet transform and Eigen-face analysis. The brass tube was tested in both air and in water, and the results demonstrate the effectiveness of the signal processing methods developed in this research. 
The simulations of acoustic propagation along the plate and tubular structures are then illustrated in Section 8. A finite element code, ABAQUS, was used to simulate the wave behavior. The frequency response was compared with the experimental data.

A summary of this research task is given in Section 9. Some concluding remarks and suggestions for future research are also outlined.

A detailed bibliography of publications related to this research task is given in the report. Some useful information may be found in the appendices. The appendices include some important mathematical operations under cylindrical coordinate system, the codes used for Lamb wave simulation, HHT and other signal processing methods, and a DAQ LABVIEW interface. 


\section{EXPERIMENTAL RESEARCH}

\subsection{Laboratory Testing System}

A smart sensor array system was developed for acoustic signal generation and data acquisition using piezo-electric sensors. Figure 2.1 illustrates the experimental modules. One piezo-sensor is used as an active sensor to generate Lamb waves in laboratory specimens, and the remaining are the passive sensors to collect the transmitted acoustic signals. The active and the passive sensors were interfaced with a PC through a standard National Instruments DAQ card. The test setup illustrated here is very important for the mode separation presented in Section 3. Figure 2.2 shows the experimental setup of a brass tube submerged in water. Here, a two-phase flow environment was simulated using air bubbles.

The active piezo-transducer is interrogated using Hanning-window modulated sine-pulses, consisting of about five cycles. The data sampling frequencies of $300 \mathrm{kHz}$ and 1.6 MHz were used. The frequency of excitation was established based on the optimal bandwidth for the specimen of interest. Active frequencies are selected in the range $13 \mathrm{kHz}-20 \mathrm{kHz}$. This frequency band was selected for two reasons. The first is the Lamb wave transportation mode in this range is much simpler than the high frequency band, so it is easy to perform the mode separation of raw signals. There is only one possible torsional mode, one longitudinal mode, and one flexural mode in this range for brass tubes. This was verified by the experimental data and is discussed in Chapters 4 and 5. The second reason is that it is located in the resonant band of the brass tubes and aluminum beams used in the experiments of this research. Hence the energy decay ratio is low. 


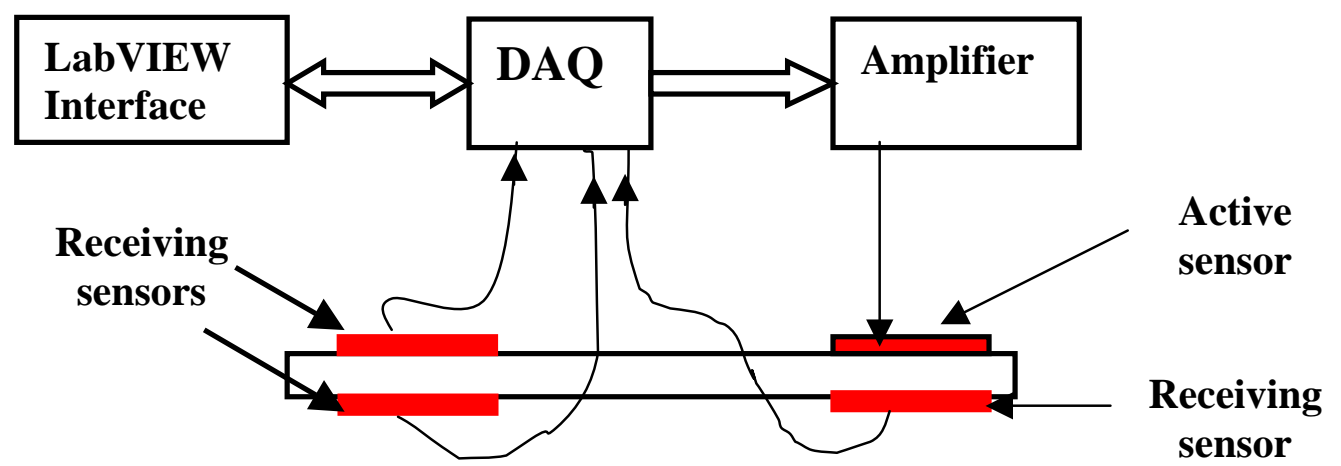

Figure 2.1. Experimental modules for interrogation of typical specimens.

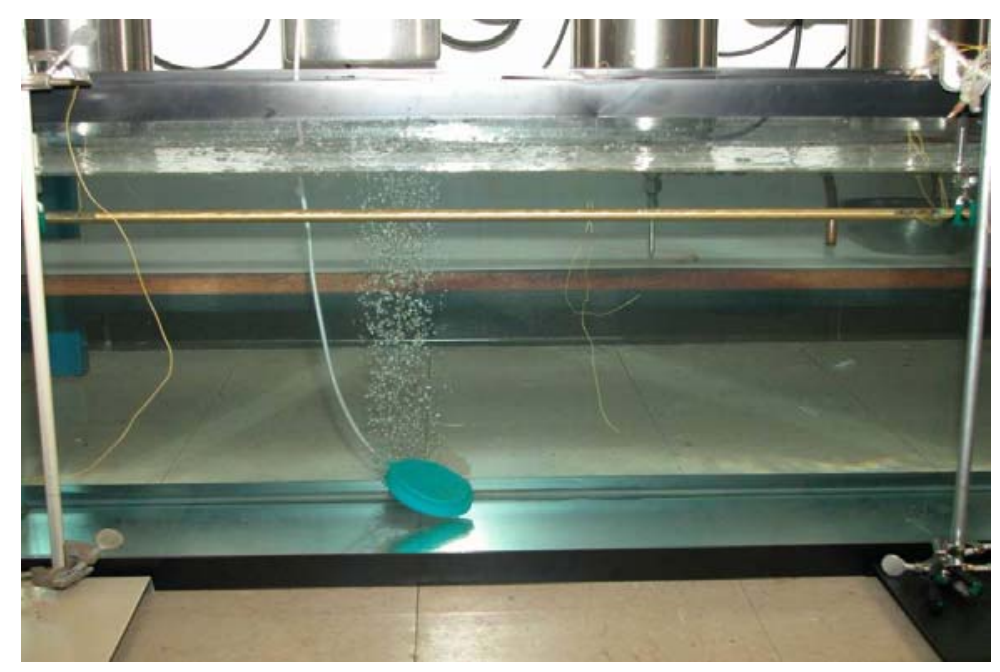

Figure 2.2. Experimental setup for testing brass tubing in water.

\subsection{Piezo-electric Materials and Piezo-sensors}

The piezoelectricity refers to the electrical polarization of crystals caused by deformation in certain directions in some materials such as quartz, tourmaline, Rochelle salt, etc. Pierre and Jacques Curie discovered this phenomenon of surface electric charges in 1880 on tourmaline crystals. It has been widely used in vibration sensors and surface acoustic wave devices in wireless signal transmission. The quick and accurate 
response of piezoelectric materials to the pressure makes it ideal for the measurement of any rapidly changing mechanical variables such as forces and accelerations. The special properties of piezoelectric materials are due to the spatial molecular crystal structures as discussed in [87]. As illustrated by Figure 2.3(a) and (b), the piezoelectric substance can be cut along different axes, the coordinate system is defined according to the crystal structure elaborated in [87]. For an X-cut piezoelectric plate, the applied pressure on the surface introduces an electrical charge on the surface. The change in the electrical field causes the plate to expand along the x-direction. The X-cut means cutting the crystal perpendicular to the $\mathrm{x}$-direction. Therefore, the periodical change of electrical field on the X-cut crystal plate generates periodical longitudinal effect inside. While for a Y-cut piezoelectric plate shown in Figure 2.3(b), the electrical filed change produces a shear force along the $\mathrm{x}$-direction, and thus creates a transverse vibration inside the plate.

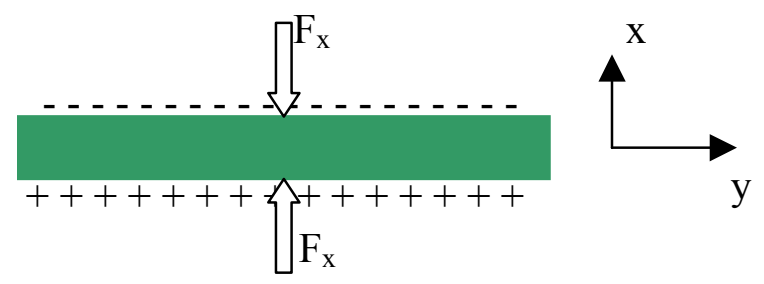

Figure 2.3(a). Longitudinal effect of piezoelectric materials with X-Cut.

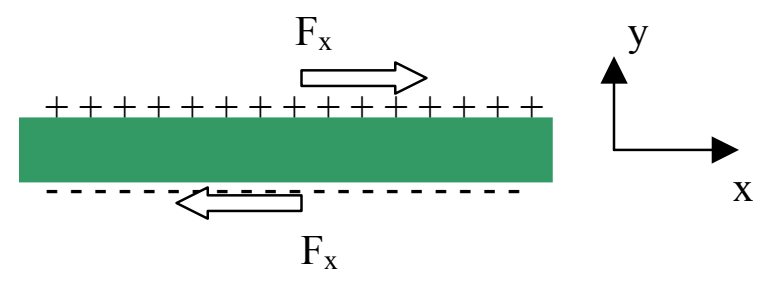

Figure 2.3(b). Shear effect of piezoelectric materials with Y-Cut.

However, the piezoelectric effect exhibited by natural materials such as quartz, and Rochelle salt, is very small, so polycrystalline ferroelectric ceramic materials such as 
barium titanate and lead (plumbum) zirconate titanate (PZT), with improved properties, have been developed. The PZT is the most widely used piezo-ceramic material nowadays. The pizeo-sensor used in this research is also made of lead, zirconate and titanate. The Curie temperature, under which the sensor should be operated, is $662{ }^{\circ} \mathrm{F}$ for this type of sensor from Piezo Systems, Inc. Notice that the primary water inlet temperature of steam generator is around $600{ }^{\circ} \mathrm{F}$, the sensor used in this research may not be suitable for on-site implementation considering the high pressure inside a SG. The high pressure may cause the decrease of stable temperature of piezoelectric materials. One solution is to use quartz slices whose stable temperature is up to $1063{ }^{\circ} \mathrm{F}$. However, this is not the issue addressed in this research.

The piezoelectric sensor sheet useed in this research is shown in Figure 2.4 (courtesy from PSI website). The thickness is 0.00105 inch and the capacity is $315 \mathrm{nf}$. The sensor sheet is then cut into small sensors useed in the experiments. Two typical sensor dimensions are: 1 inch x 0.375 inch for aluminum beam; $1.5 \times 0.1875$ inch for brass tubes. These sensors are shown in Figure 2.5. The figure also shows some structural flaws tested in this research.

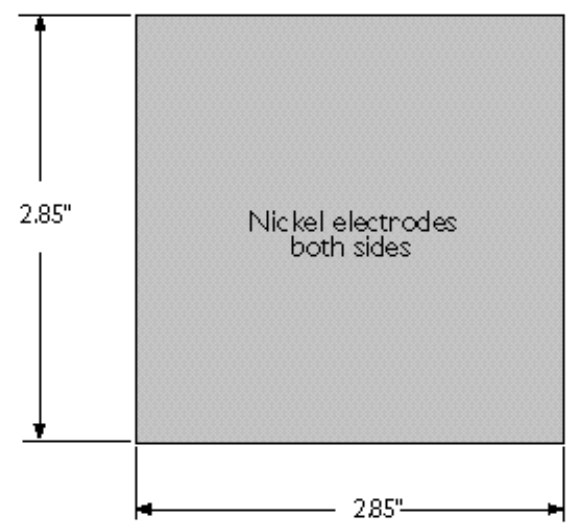

Figure 2.4. Piezoelectric sensor sheet. Thickness: 0.00105 inch (from PSI).

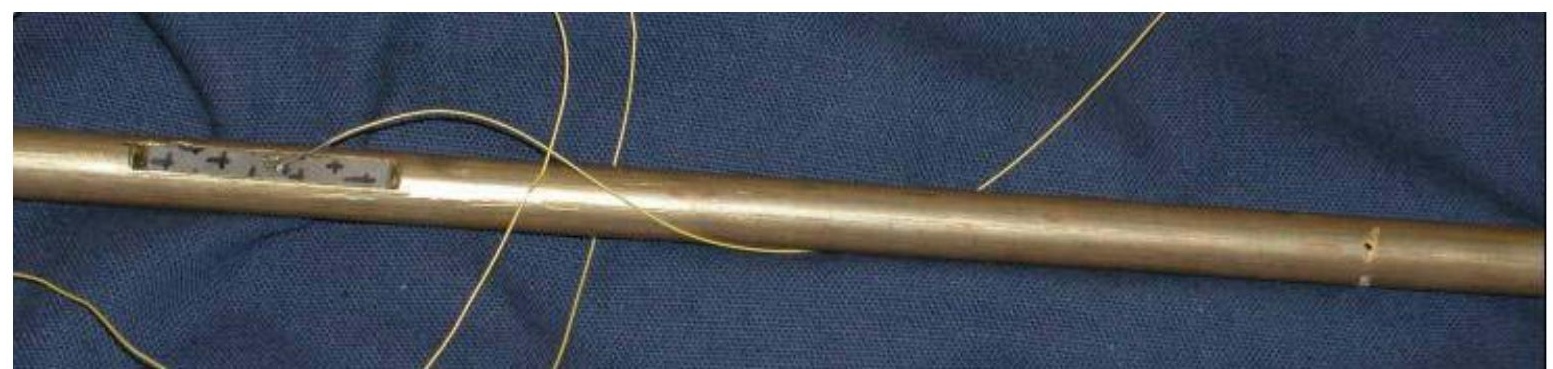


(a)

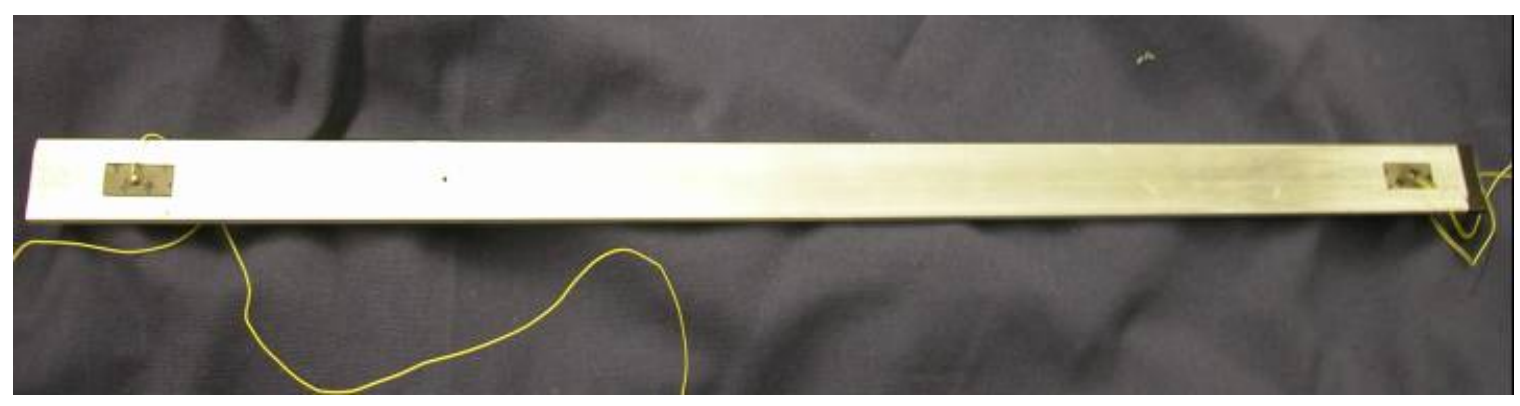

(b)

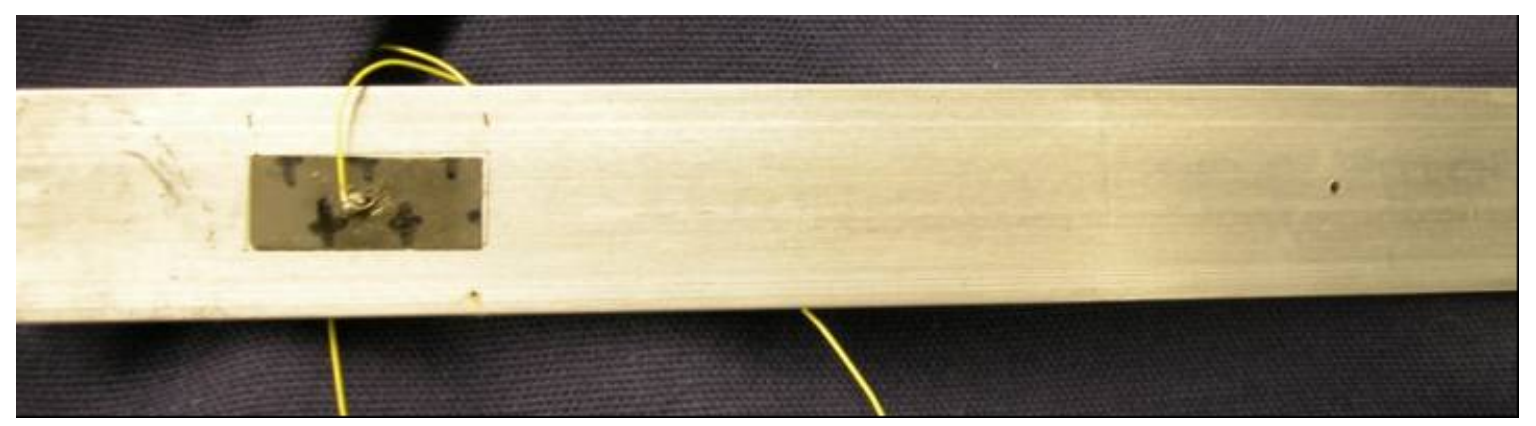

(c)

Figure 2.5. Experimental specimens with sensor, and structural flaw. (a) Brass tube; (b) Aluminum plate; (c) Partial beam. 


\subsection{Activation of Guided Acoustics Using Piezo-sensors}

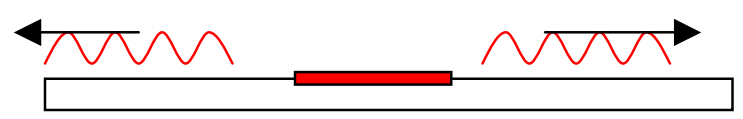

(a)

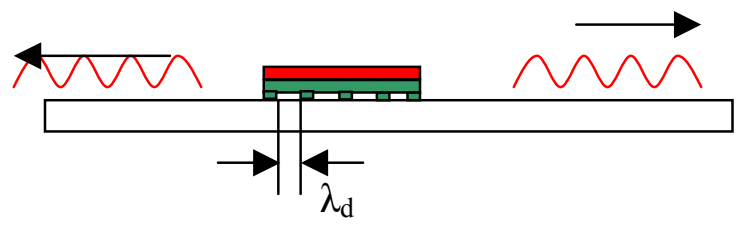

(b)

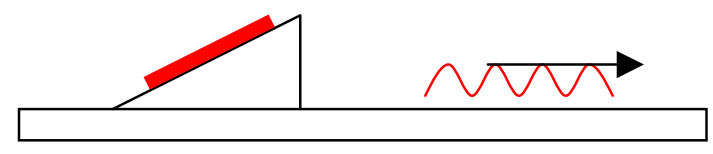

(c)

Figure 2.6. Methods of Lamb wave generation.

There are several commonly adopted methods for Lamb wave generation as discussed by Viktorov in [22]. These methods are illustrated in Figure 2.6. In Figure 2.6(a), the piece of piezoelectric transducer is directly bonded onto a plate. The Lamb waves are then generated as the electrical field applied on the sensor changes. The generated Lamb waves propagate along the plate toward two opposite directions. All possible transportation modes, based on the input frequency, will be excited through this type of sensor setup. The advantage of this method is the simplicity of sensor installation and manufacturing. The disadvantage is that Lamb waves generated are fairly complicated, especially in the high frequency band. Since the space available in steam generators is limited, this method is utilized in this project in order to simplify the instrumentation. The disadvantages will be overcome by the careful selection of the input signal band and the mode separation technique discussed in Section 3. 
Another Lamb wave excitation method is illustrated in Figure 2.6(b). A piece of piezo-transducer (X-cut) is placed on a piece of metal plate with corrugated, combshaped profile on one side. The slot width of the comb profile is $\lambda_{\mathrm{d}}$, which decides the wavelength of the guided acoustics generated by this structure. The Lamb wavelength would be $\lambda=2 \lambda_{d}$. An important advantage of this method is that the wavelength is selectively decided by the slot width and thus it is easy to determine the resonant input frequency from the dispersion curves. The dispersion curve in Figure 2.7 is the numerical solution of Lamb wave propagation along an aluminum plate that discussed in Section 3. The intersection points of a line with a gradient of $\lambda / d$ and the dispersion curves decide the resonant input frequencies as shown in the figure with circles. This method can effectively activate Lamb waves in almost any elastic material. But it is not used in the experiments of this research because the frequency band adopted here is not very high, thus the wavelength is too long for the comb structure considering the small dimension of the experimental specimen. Nevertheless this method has great potential for high frequency Lamb wave implementation in long tubes such as oil pipes.

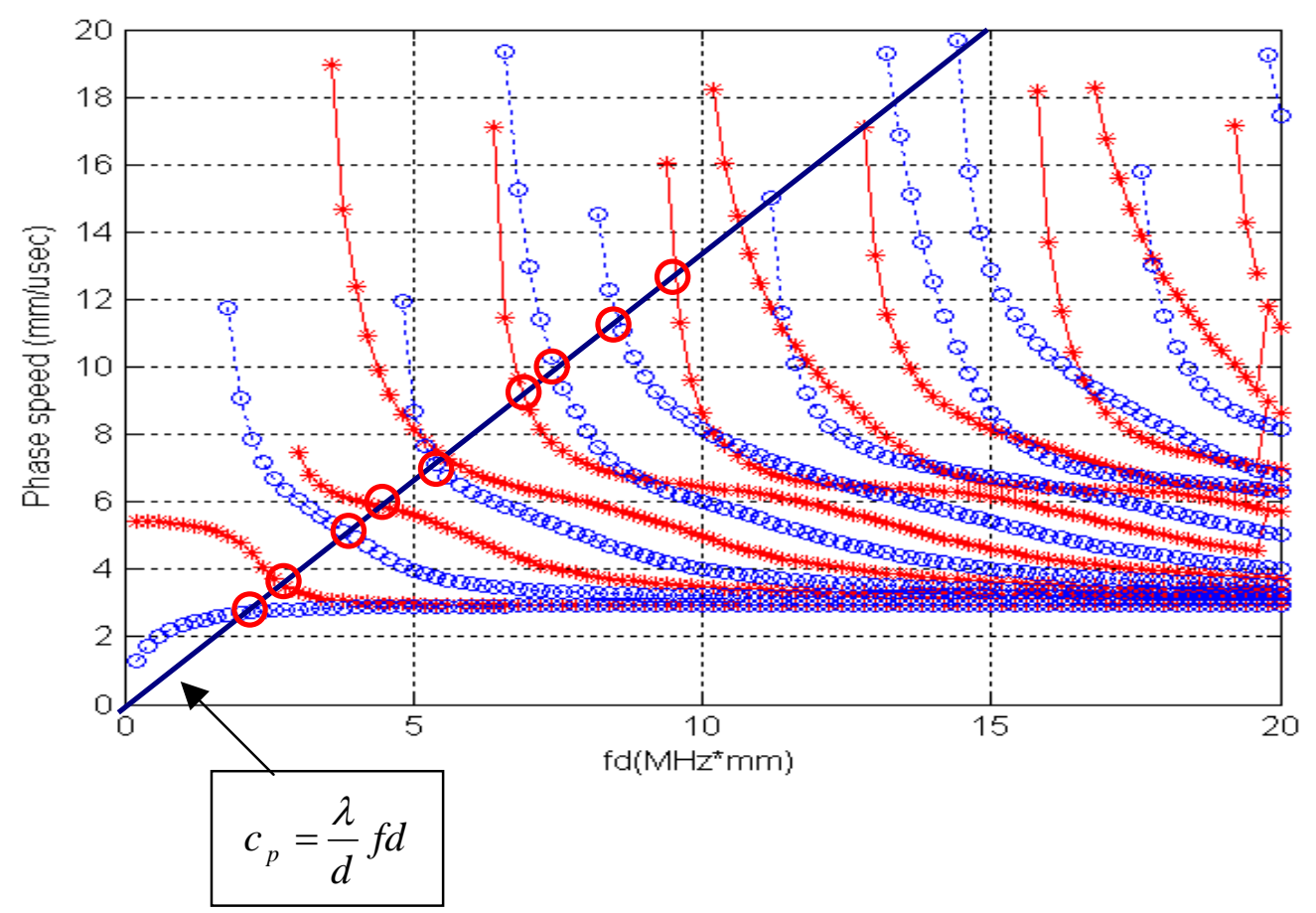

Figure 2.7. The resonant frequency is decided by slot width $d$ and the Lamb wave mode. 
The third method is called wedge technique as illustrated in Figure 2.6(c). A wedge block, usually made of plastic, is placed on the experimental specimen. A piezotransducer generates longitudinal waves in the wedge. The longitudinal waves then convert into Lamb waves in the experimental specimen. A modified method uses Y-cut piezoelectric plate to generate transverse waves in the wedge block. Different Lamb mode signals may be activated by the adjustment of the wedge angle. As the most widely used method, wedge block method has been extensively explored for the study of ultrasonic testing. The advantage of this method is the flexibility in the selective generation of Lamb waves at a given frequency. However, it is not as efficient as the comb structure discussed above, and its setup is not suitable for online monitoring of SG tubes due to the limited available space; hence it is not considered in this research. 


\section{FUNDAMENTALS OF LAMB WAVE THEORY}

\subsection{Elastic Wave Propagation Along Thin Plates}

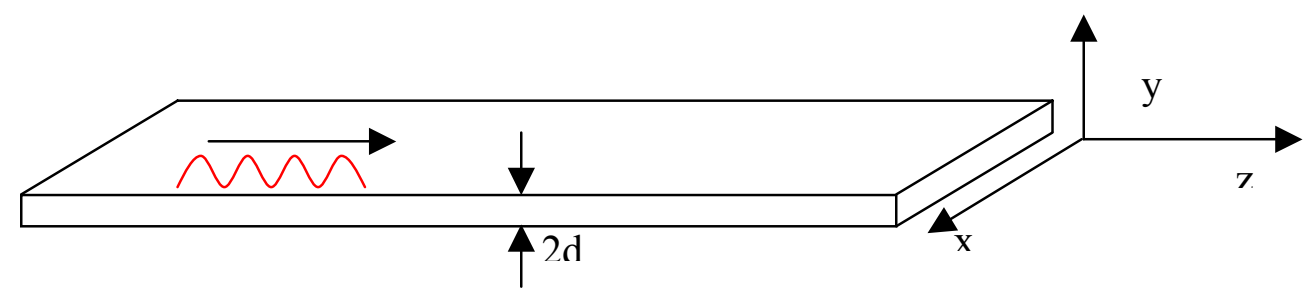

Figure 3.1. Guided acoustic waves in a plate-like structure.

Considering the propagation along a thin plate, as in Figure 3.1, Lamb wave is a special type of surface wave, for which the displacements occur in the direction of both its propagation and normal to the free boundaries. The stress components on the boundaries must be zero. The general particle displacement equation $[22,30]$ can be written in the potential form as:

$$
\begin{aligned}
& \vec{u}=\nabla \phi+\nabla \times(\vec{\psi}) \\
& \nabla \bullet(\vec{\psi})=0 \\
& \phi: \text { scalar potential of longitudal wave } \\
& \vec{\psi}: \text { vector potential of transverse wave } \\
& \vec{u}: \text { particle displacement. }
\end{aligned}
$$

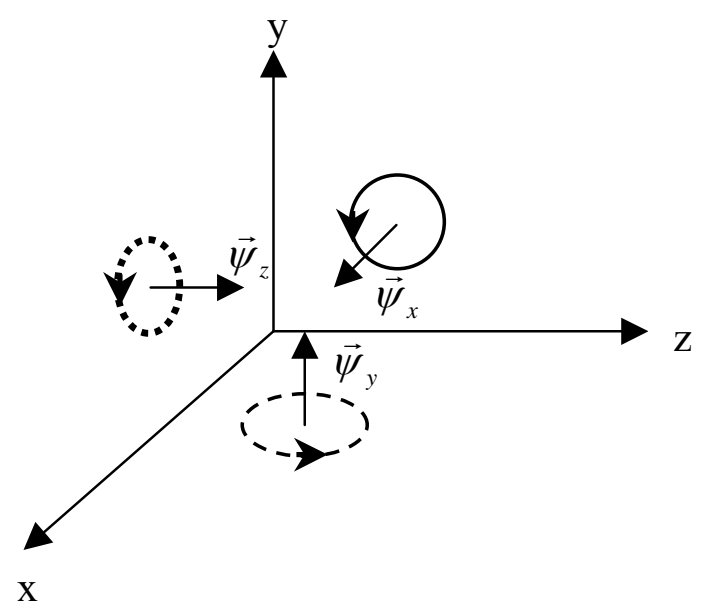

Figure 3.2. Vector potentials and particle movement. 
The condition of $\nabla \bullet(\vec{\psi})=0$ is necessary to make sure that the particles with same $\mathrm{x}$-coordinate are rotating around its balance position in a close route in the same direction and same speed. As demonstrated in figure 3.2, the particle displacements caused by the vector potential is:

$$
\vec{v}=\nabla \times \vec{\psi}=\left|\begin{array}{ccc}
\vec{x} & \vec{y} & \vec{z} \\
\frac{\partial}{\partial x} & \frac{\partial}{\partial y} & \frac{\partial}{\partial z} \\
\psi_{x} & \psi_{y} & \psi_{z}
\end{array}\right|=\left\{\begin{array}{c}
\left(\frac{\partial \psi_{z}}{\partial y}-\frac{\partial \psi_{y}}{\partial z}\right) \vec{x} \\
\left(-\frac{\partial \psi_{z}}{\partial x}+\frac{\partial \psi_{x}}{\partial z}\right) \vec{y} \\
\left.\frac{\partial \psi_{y}}{\partial x}-\frac{\partial \psi_{x}}{\partial y}\right) \vec{z}
\end{array}\right.
$$

$\vec{v}:$ particle displacement caused by $\vec{\psi}$.

By assuming that the displacement along the $\mathrm{x}$-axis is zero, we can derive that the vector potential has only the nonzero component in the direction of $\mathrm{x}$, i.e. $\psi_{y}=\psi_{z}=0$. Let $\psi_{x}=\varphi$, we can rewrite the wave equations as $[3,22]$

$$
\begin{aligned}
& \frac{\partial^{2} \phi}{\partial z^{2}}+\frac{\partial^{2} \phi}{\partial y^{2}}+k_{l}^{2} \phi=0 \\
& \frac{\partial^{2} \varphi}{\partial z^{2}}+\frac{\partial^{2} \varphi}{\partial y^{2}}+k_{t}^{2} \varphi=0
\end{aligned}
$$

The solutions of these equations have the following form:

$$
\begin{aligned}
& \phi=A * \operatorname{ch}\left(y \sqrt{k^{2}-k_{l}^{2}}\right) e^{i(k z-\omega t)}+B * \operatorname{sh}\left(y \sqrt{k^{2}-k_{l}^{2}}\right) e^{i(k z-\omega t)}, \\
& \varphi=C * \operatorname{sh}\left(y \sqrt{k^{2}-k_{t}^{2}}\right) e^{i(k z-\omega t)}+D * \operatorname{ch}\left(y \sqrt{k^{2}-k_{t}^{2}}\right) e^{i(k z-\omega t)} .
\end{aligned}
$$

$\mathrm{A}, \mathrm{B}, \mathrm{C}$, and $\mathrm{D}$ are constants. All terms in the equations have the same complex exponential term $e^{i(k z-\omega t)}$, which is time dependent and influences the wave speed in the $\mathrm{z}$-direction. The remaining terms in the equations are only related to $\mathrm{y}$ and are time independent. 


$$
\begin{aligned}
& \phi^{\prime}=A * \operatorname{sh}\left(y \sqrt{k^{2}-k_{l}^{2}}\right)+B * \operatorname{ch}\left(y \sqrt{k^{2}-k_{l}^{2}}\right), \\
& \varphi^{\prime}=C * \operatorname{sh}\left(y \sqrt{k^{2}-k_{t}^{2}}\right)+D * \operatorname{ch}\left(y \sqrt{k^{2}-k_{t}^{2}}\right) .
\end{aligned}
$$

Rewrite the above using sine and cosine functions:

$$
\begin{aligned}
& \phi^{\prime}=A^{\prime} * \sin \left(y \sqrt{k_{l}^{2}-k^{2}}\right)+B^{\prime} * \cos \left(y \sqrt{k_{l}^{2}-k^{2}}\right), \\
& \varphi^{\prime}=C^{\prime} * \sin \left(y \sqrt{k_{t}^{2}-k^{2}}\right)+D^{\prime} * \cos \left(y \sqrt{k_{t}^{2}-k^{2}}\right),
\end{aligned}
$$

where $\phi^{\prime}$ and $\varphi^{\prime}$ represent the standing waves in the y-direction (thickness). The displacement and the stress functions in the thin plate can then be written as follows:

$$
\begin{aligned}
& u_{x}=0, \\
& u_{y}=\frac{\partial \phi}{\partial y}-\frac{\partial \varphi}{\partial z}=\frac{d \phi^{\prime}}{d y}-i k \varphi^{\prime}, \\
& u_{z}=\frac{\partial \phi}{\partial z}+\frac{\partial \varphi}{\partial y}=i k \phi^{\prime}+\frac{d \varphi^{\prime}}{d y}, \\
& \sigma_{y z}=\mu\left(\frac{\partial u_{y}}{\partial z}+\frac{\partial u_{z}}{\partial y}\right)=\mu\left(2 i k \frac{d \phi^{\prime}}{d y}+k^{2} \varphi^{\prime}+\frac{d^{2} \varphi^{\prime}}{d y^{2}}\right), \\
& \sigma_{y y}=\lambda\left(\frac{\partial u_{z}}{\partial z}+\frac{\partial u_{y}}{\partial y}\right)+2 \mu \frac{\partial u_{y}}{\partial y}=\lambda\left(-k^{2} \phi^{\prime}+\frac{d^{2} \phi^{\prime}}{d y^{2}}\right) \\
& +2 \mu\left(\frac{d^{2} \phi^{\prime}}{d y^{2}}-i k \frac{d^{2} \varphi^{\prime}}{d y^{2}}\right),
\end{aligned}
$$

Where:

$u_{x, y, z}:$ particle displacement,

$\sigma_{y,[z, y]}:$ stress,

$\lambda, \mu:$ Lame constant.

We are able to separate them into symmetric and anti-symmetric terms according to the displacement in the direction of $y$ as follows: 
Symmetric modes:

$$
\begin{aligned}
& \phi^{\prime}=B^{\prime} \cos \left(y \sqrt{k_{l}^{2}-k^{2}}\right), \\
& \varphi^{\prime}=C^{\prime} \sin \left(y \sqrt{k_{t}^{2}-k^{2}}\right), \\
& u_{y}=-B^{\prime} \sqrt{k_{l}^{2}-k^{2}} \cos \left(y \sqrt{k_{l}^{2}-k^{2}}\right)-i k C^{\prime} \cos \left(y \sqrt{k_{t}^{2}-k^{2}}\right), \\
& u_{z}=i k B^{\prime} \sin \left(y \sqrt{k_{l}^{2}-k^{2}}\right)+\sqrt{k_{t}^{2}-k^{2}} C^{\prime} \sin \left(y \sqrt{k_{t}^{2}-k^{2}}\right), \\
& \sigma_{y z}=\mu\left(-2 i k B^{\prime} \sqrt{k_{l}^{2}-k^{2}} \sin \left(y \sqrt{k_{l}^{2}-k^{2}}\right)+C^{\prime}\left(2 k^{2}-k_{t}^{2}\right) \sin \left(y \sqrt{k_{t}^{2}-k^{2}}\right)\right), \\
& \sigma_{y y}=-\lambda k_{l}^{2} B^{\prime} \cos \left(y \sqrt{k_{l}^{2}-k^{2}}\right)-2 \mu\left(\left(k_{l}^{2}-k^{2}\right) B^{\prime} \cos \left(y \sqrt{k_{l}^{2}-k^{2}}\right),\right. \\
& +i k C^{\prime} \sqrt{k_{t}^{2}-k^{2}} \cos \left(y \sqrt{k_{t}^{2}-k^{2}}\right) .
\end{aligned}
$$

For the symmetric mode, the particle displacement in the z-direction is symmetric across the thickness of the plate, while the displacement in the y-direction is anti-symmetric.

Anti-symmetric modes:

$$
\begin{aligned}
& \phi^{\prime}=A^{\prime} \sin \left(y \sqrt{k_{l}^{2}-k^{2}}\right), \\
& \varphi^{\prime}=D^{\prime} \cos \left(y \sqrt{k_{t}^{2}-k^{2}}\right), \\
& u_{y}=-i k A^{\prime} \sin \left(y \sqrt{k_{l}^{2}-k^{2}}\right)-D^{\prime} \sqrt{k_{t}^{2}-k^{2}} \sin \left(y \sqrt{k_{t}^{2}-k^{2}}\right), \\
& u_{z}=A^{\prime} \sqrt{k_{l}^{2}-k^{2}} \cos \left(y \sqrt{k_{l}^{2}-k^{2}}\right)-i k D^{\prime} \cos \left(y \sqrt{k_{t}^{2}-k^{2}}\right), \\
& \sigma_{y z}=\mu\left(-2 i k A^{\prime} \sqrt{k_{l}^{2}-k^{2}} \cos \left(y \sqrt{k_{l}^{2}-k^{2}}\right)+D^{\prime}\left(2 k^{2}-k_{t}^{2}\right) \cos \left(y \sqrt{k_{t}^{2}-k^{2}}\right)\right), \\
& \sigma_{y y}=-\lambda k_{l}^{2} A^{\prime} \sin \left(y \sqrt{k_{l}^{2}-k^{2}}\right)-2 \mu\left(\left(k_{l}^{2}-k^{2}\right) A^{\prime} \sin \left(y \sqrt{k_{l}^{2}-k^{2}}\right)\right. \\
& -i k D^{\prime} \sqrt{k_{t}^{2}-k^{2}} \cos \left(y \sqrt{k_{t}^{2}-k^{2}}\right) .
\end{aligned}
$$

For the anti-symmetric mode, the particle displacement in the z-direction is antisymmetric across the thickness of the plate, while the displacement in the y-direction is symmetric.

Using boundary conditions that the stress at free boundaries must be zero, 


$$
\begin{aligned}
& \sigma_{y z}=0 \text { at } y= \pm \frac{d}{2}, \\
& \sigma_{y y}=0 \text { at } y= \pm \frac{d}{2}, \\
& d: \text { thickness, }
\end{aligned}
$$

we can write out two characteristic equations to determine the wave number k:

$$
\begin{aligned}
& \text { Sym }: \frac{\tan \sqrt{1-\zeta^{2}} \hat{d}}{\tan \sqrt{\gamma^{2}-\zeta^{2}} \hat{d}}=-\frac{4 \zeta^{2} \sqrt{1-\zeta^{2}} \sqrt{\gamma^{2}-\zeta^{2}}}{\left(2 \zeta^{2}-1\right)^{2}}, \\
& \text { Anti }: \frac{\tan \sqrt{1-\zeta^{2}} \hat{d}}{\tan \sqrt{\gamma^{2}-\zeta^{2}} \hat{d}}=-\frac{\left(2 \zeta^{2}-1\right)^{2}}{4 \zeta^{2} \sqrt{1-\zeta^{2}} \sqrt{\gamma^{2}-\zeta^{2}}},
\end{aligned}
$$

Where

$$
\begin{aligned}
& \hat{d}=k_{t} d ; \zeta^{2}=\frac{c_{t}^{2}}{c^{2}} ; \gamma=\frac{c_{t}^{2}}{c_{l}^{2}}, \\
& d: \text { thickness of plate, } \\
& c_{t}: \text { transverse wave speed, } \\
& c_{l}: \text { longitudinal wave speed, } \\
& c: \text { phase speed of Lamb wave, } \\
& k_{t}=\frac{\omega}{c_{t}} \text { : transverse wave number, } \\
& \omega: \text { frequency. }
\end{aligned}
$$

From these equations we are able to obtain solutions of wave numbers $k_{s}$ and $k_{a}$ (symmetric and anti-symmetric).

The numerical solutions of the above characteristic equations are shown in Figure 3.3. The relationship between the wave number $\mathrm{k}$ and the phase speed is defined as

$$
\begin{aligned}
& k=\frac{\omega}{c_{p}}, \\
& c_{p}: \text { phase speed } .
\end{aligned}
$$




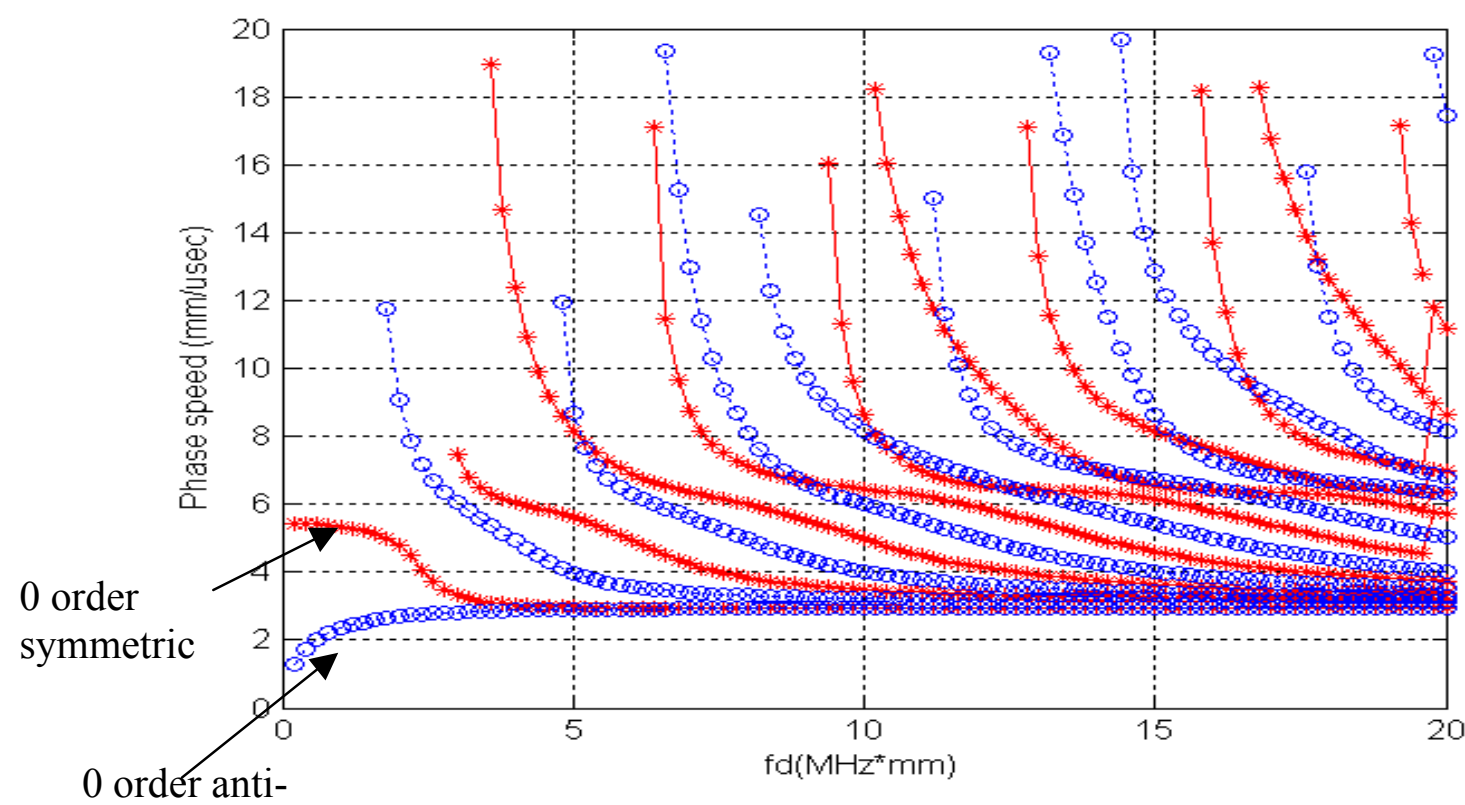

order anti-

Figure 3.3. Dispersion curves for a traction free aluminum beam.

The Lamb wave transportation becomes more complicated as the frequency increases for a plate with fixed thickness. But there is only one symmetric and one anti-symmetric mode for low frequency waves.

Unfortunately the Lamb waves usually propagate in a group, i.e. many modes are mixed together. Hence the phase speed cannot describe the behavior of Lamb wave propagation unless a pure mode is generated in a plate. Therefore the group speed is introduced and defined as

$$
c_{g}=\frac{d \omega}{d k}=\frac{d \omega}{d\left(\frac{\omega}{c_{p}}\right)}=\frac{d \omega}{\frac{d \omega}{c_{p}}-\omega \frac{d c_{p}}{c_{p}^{2}}}=\frac{c_{p}^{2}}{c_{p}-\omega \frac{d c_{p}}{d \omega}}=\frac{c_{p}^{2}}{c_{p}-f d \frac{d c_{p}}{d(f d)}},
$$

where : $\omega=2 \pi f d$. 
The numerical solution of the group speed as a function of the product of frequency and thickness is plotted in Figure 3.4.

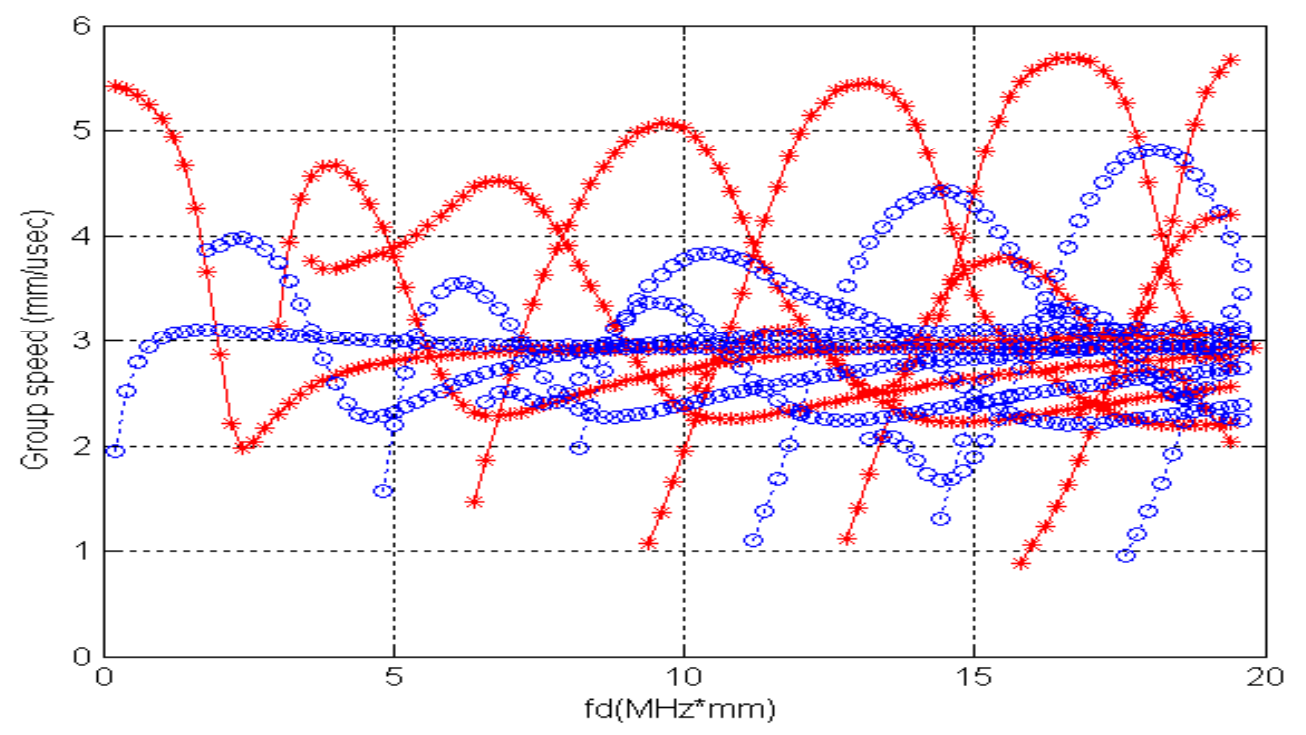

Figure 3.4. Group speed of Lamb waves in a traction free aluminum beam.

It is interesting to see that the phase speed of each mode is very close to its phase speed in the low frequency band. This makes the problem of Lamb wave transportation easier as long as the input signal has low frequency.

Similarly, the more complicated Lamb wave properties in metal tubes have been derived [22, 30, 89]. In summary, it is found that Lamb waves propagate in multiple modes. Hence the signals from the transducers are dependent on their positions, wave frequency, and plate thickness. An important property related to the defect inspection is that a single input mode signal will be scattered forward and backward as in a multimode propagation. The structural flaw may be viewed as a new source of waves being scattered around. Its presence would definitely alter the signal characteristics and its frequency spectrum, changes of which are useful for nondestructive examination. 


\subsection{Elastic Waves in Metal Tubes}

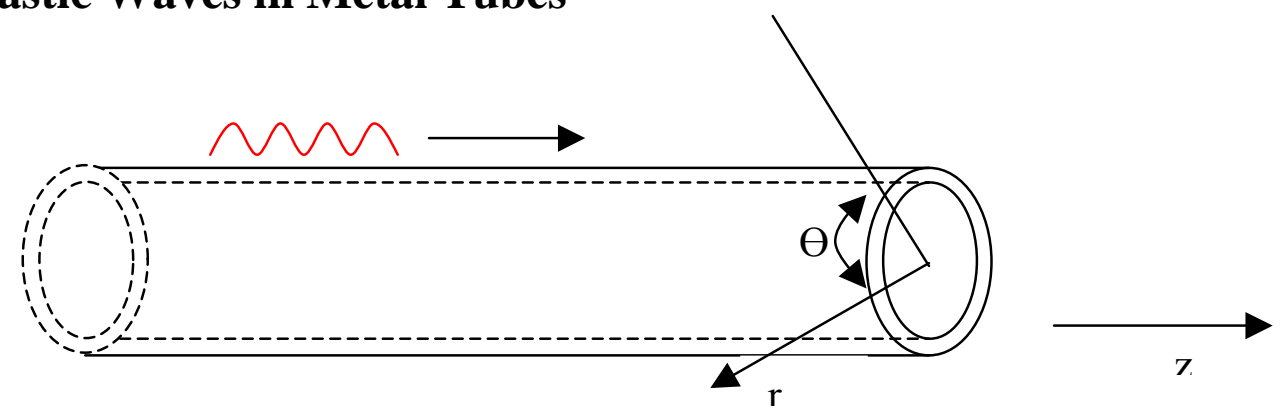

Figure 3.5. Guided acoustic waves in tubing.

Lamb wave signals in tubing structures are more complicated because they are mixtures of time series with different modes. In addition, the spread of frequency will cause the spread of wave speed even using a simple sine pulse as input, and further increases the difficulty in analyzing tubular Lamb waves. Many studies have been performed in the propagation of Lamb waves in tubular structures. In summary, there are four types of Lamb wave modes in tubular structures. Each of them is described.

- Circumferential - non-propagation mode:

A circumferential mode is a type of wave that transports around in the circumferential direction; thus, it is a non-propagating mode in the axial direction. This type of mode is not very useful for structural monitoring unless the defect is located at the same axial position and different circumferential locations as the active sensor. The wave propagation is illustrated in Figure 3.6a.

- Flexural modes - anti-symmetrical modes:

As shown in Figure 3.6b, the flexural modes are anti-symmetric modes whose axial particle displacements are anti-symmetric with respect to the central line of the tube. Therefore, if two sensors are deployed at the same axial position on the tube and have 180-degree apart in the circumferential direction, the signals collected should have 180degree phase difference. This is important for mode separation. As illustrated later, the flexural mode plays a key role in flaw detection and isolation for tube-like structures.

- Longitudinal-symmetrical modes: 
The wave propagation of longitudinal modes is illustrated in Figure 3.6c. The particle displacement in the axial direction is symmetric across the structure. The experimental data of this research demonstrated the significance of symmetrical modes.

- Torsional-symmetrical modes:

Another type of symmetrical modes is the torsional mode shown in Figure 3.6d. The properties such as dispersion curves will be illustrated later in this section and can also be found in references [22, 30,89]. This type of mode is also important in this research because of its presence in the experimental data.

Because of the complexity of Lamb waves in tubes, advanced non-stationary signal processing methods are necessary for understanding Lamb wave properties, including fault detection, structural flaw evaluation, and classification. 


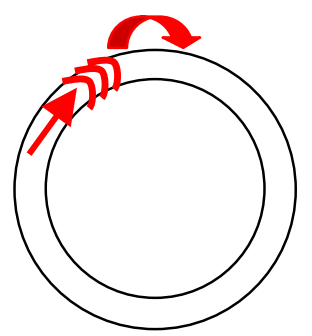

(a)

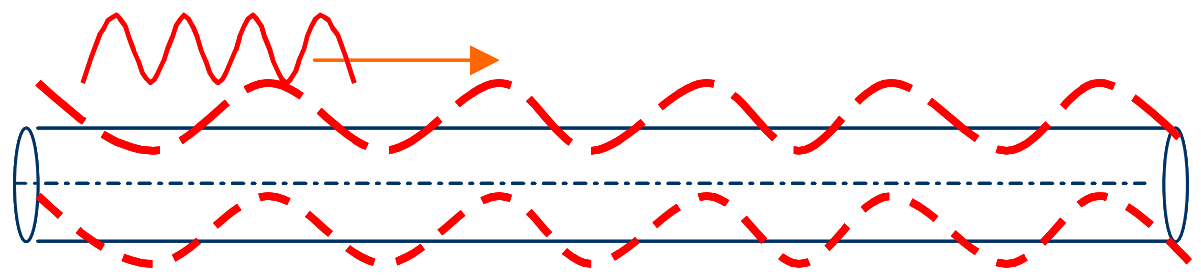

(b)

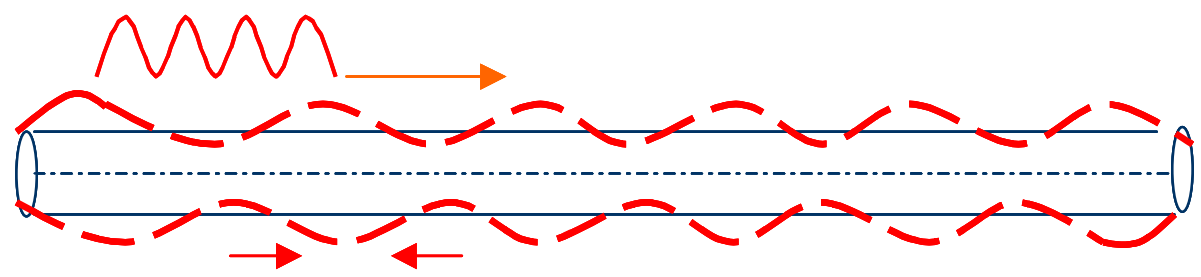

(c)

\section{Torsion}

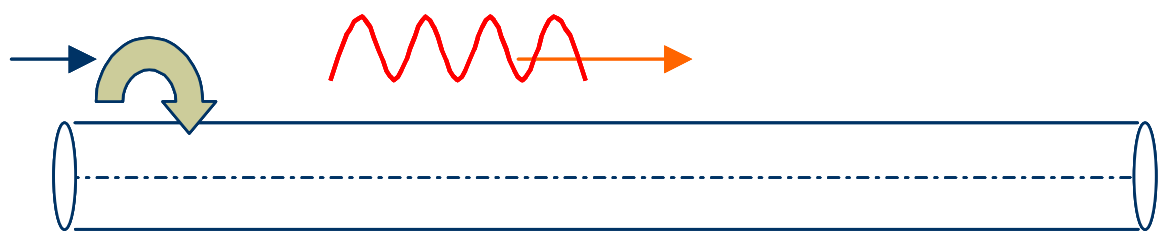

(d)

Figure 3.6. Lamb wave modes in tubular structures.

For a hollow cylinder, the circumferential wave equations in terms of the potentials $\phi$ and $\psi$ is written as:

$$
\begin{aligned}
& \left(\frac{\partial^{2}}{\partial r^{2}}+\frac{1}{r} \frac{\partial}{\partial r}+\frac{1}{r^{2}} \frac{\partial^{2}}{\partial \theta^{2}}\right) \phi+\frac{\omega^{2}}{c_{L}^{2}} \phi=0 \\
& \left(\frac{\partial^{2}}{\partial r^{2}}+\frac{1}{r} \frac{\partial}{\partial r}+\frac{1}{r^{2}} \frac{\partial^{2}}{\partial \theta^{2}}\right) \psi_{z}+\frac{\omega^{2}}{c_{T}^{2}} \psi_{z}=0
\end{aligned}
$$

Where $c_{\mathrm{L}}$ and $\mathrm{c}_{\mathrm{T}}$ are longitudinal and shear wave velocities, respectively. The general solution is given by 


$$
\begin{aligned}
& \phi(r)=A_{1} J_{k b}\left(\frac{\omega r}{C_{L}}\right)+A_{2} Y_{k b}\left(\frac{\omega r}{c_{L}}\right) \\
& \psi_{z}(r)=A_{3} J_{k b}\left(\frac{\omega r}{c_{T}}\right)+A_{4} Y_{k b}\left(\frac{\omega r}{c_{T}}\right)
\end{aligned}
$$

Where $\mathrm{J}_{\mathrm{kb}}(\mathrm{z})$ and $\mathrm{Y}_{\mathrm{kb}}(\mathrm{z})$ are the first and second type Bessel functions, respectively. A1, $\mathrm{A} 2, \mathrm{~A} 3$, and A4 are constants.

The particle displacements and stresses can be represented in the form of potentials as:

$$
\begin{gathered}
u_{r}=\frac{\partial \phi}{\partial r}+\frac{1}{r} \frac{\partial \psi}{\partial \theta}, \\
u_{\theta}=\frac{1}{r} \frac{\partial \phi}{\partial \theta}-\frac{\partial \psi}{\partial r}, \\
\sigma_{r r}=\lambda\left(\frac{\partial u_{r}}{\partial r}+\frac{u_{r}}{r}+\frac{1}{r} \frac{\partial u_{\theta}}{\partial \theta}\right)+2 \mu \frac{\partial u_{r}}{\partial r}, \\
\sigma_{r \theta}=\lambda\left(\frac{\partial u_{\theta}}{\partial r}-\frac{u_{\theta}}{r}+\frac{1}{r} \frac{\partial u_{r}}{\partial \theta}\right) . \\
\sigma_{r r}=(\lambda+2 \mu) \frac{\partial^{2} \phi}{\partial r^{2}}+\frac{\lambda}{r} \frac{\partial \phi}{\partial r}+\frac{\lambda}{r^{2}} \frac{\partial^{2} \phi}{\partial \theta^{2}}-\frac{2 \mu}{r^{2}} \frac{\partial \psi}{\partial \theta}+\frac{2 \mu}{r} \frac{\partial^{2} \psi}{\partial \theta \partial r} \\
\sigma_{r \theta}=\mu\left(-\frac{2}{r^{2}} \frac{\partial \phi}{\partial \theta}+\frac{2}{r} \frac{\partial^{2} \phi}{\partial r \partial \theta}-\frac{\partial^{2} \psi}{\partial r^{2}}+\frac{1}{r} \frac{\partial \psi}{\partial r}+\frac{1}{r^{2}} \frac{\partial^{2} \psi}{\partial^{2} \theta}\right) . \\
\phi(r, \theta)=\varphi(r) e^{i(k b \theta-\omega t)}, \\
\psi(r, \theta)=\vartheta(r) e^{i(k b \theta-\omega t)},
\end{gathered}
$$

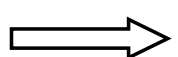

Equations (3.18) then yield the following: 


$$
\begin{aligned}
& \sigma_{r r}=e^{i(i b \theta+\omega t)}\left\{(\lambda+2 \mu) \frac{\partial^{2} \varphi}{\partial r^{2}}+\frac{\lambda}{r} \frac{\partial \varphi}{\partial r}-k^{2} b^{2} \frac{\lambda}{r^{2}} \varphi-\frac{2 \mu}{r^{2}} i k b \vartheta+\frac{2 \mu}{r} i k b \frac{\partial \psi}{\partial r}\right\}, \\
& \sigma_{r \theta}=\frac{\mu}{r^{2}} \mu e^{i(i b \theta+\omega t)}\left\{-2 i k b \varphi+2 r i k b \frac{\partial \varphi}{\partial r}-r^{2} \frac{\partial^{2} \vartheta}{\partial r^{2}}+r \frac{\partial \vartheta}{\partial r}-k^{2} b^{2} \vartheta\right\}, \\
& \text { define }: \chi=\frac{\lambda+2 \mu}{\mu}=\frac{c^{2} l}{c^{2}{ }_{t}}, \\
& \sigma_{r r}=\frac{\mu}{r^{2}} e^{i(i b \theta+\omega t)}\left\{\chi \frac{\partial^{2} \varphi}{\partial r^{2}}+r(\chi-2) \frac{\partial \varphi}{\partial r}-(\chi-2) k^{2} b^{2} \varphi-2 i k b \vartheta+2 i k b r \frac{\partial \psi}{\partial r}\right\}, \\
& \sigma_{r \theta}=\frac{\mu}{r^{2}} \mu e^{i(i b \theta+\omega t)}\left\{-2 i k b \varphi+2 r i k b \frac{\partial \varphi}{\partial r}-r^{2} \frac{\partial^{2} \vartheta}{\partial r^{2}}+r \frac{\partial \vartheta}{\partial r}-k^{2} b^{2} \vartheta\right\} .
\end{aligned}
$$

Using the boundary conditions similar to plate structure described above

$$
\begin{aligned}
& \sigma_{r r}=0, \\
& \sigma_{r \theta}=0 . \\
& \text { for } r=a, b, \\
& a: \text { inner tube radius } \\
& b: \text { outer tube radius. }
\end{aligned}
$$

The following equations show the recursive relations for Bessel functions.

$$
\begin{aligned}
& \frac{2 n}{z} J_{n}(x)=J_{n-1}(x)+J_{n+1}(x), \\
& 2 \frac{d J_{n}(x)}{d x}=J_{n-1}(x)-J_{n+1}(x), \\
& x J_{n-1}(x)=n J_{n}(x)+x \frac{d J_{n}(x)}{d x}, \\
& -x J_{n+1}(x)=-n J_{n}(x)+x \frac{d J_{n}(x)}{d x} .
\end{aligned}
$$

Note: Same relations exist for the second type Bessel functions $\mathrm{Y}_{\mathrm{n}}(\mathrm{x})$.

The four constants, A1, A2, A3 and A4 in Equation (3.16) are determined by setting the determinant of the system equations to zero. The characteristic equations are derived as: 
$\left\|D_{i j}\right\|=0, \quad i, j=1: 4$

where,

$$
\begin{aligned}
& D_{11}=\left[J_{k b-2}\left(\frac{\omega b}{c_{l}}\right)+J_{k b+2}\left(\frac{\omega b}{c_{l}}\right)-2(\chi-1) J_{k b}\left(\frac{\omega b}{c_{l}}\right)\right] \chi^{-1}, \\
& D_{12}=i\left[J_{k b-2}\left(\frac{\omega b}{c_{t}}\right)-J_{k b+2}\left(\frac{\omega b}{c_{t}}\right)\right], \\
& D_{13}=\left[Y_{k b-2}\left(\frac{\omega b}{c_{l}}\right)+Y_{k b+2}\left(\frac{\omega b}{c_{l}}\right)-2(\chi-1) Y_{k b}\left(\frac{\omega b}{c_{l}}\right)\right] \chi^{-1}, \\
& D_{14}=i\left[Y_{k b-2}\left(\frac{\omega b}{c_{t}}\right)-Y_{k b+2}\left(\frac{\omega b}{c_{t}}\right)\right], \\
& D_{21}=i\left[J_{k b-2}\left(\frac{\omega b}{c_{l}}\right)-J_{k b+2}\left(\frac{\omega b}{c_{l}}\right)\right] \chi^{-1}, \\
& D_{22}=-\left[J_{k b-2}\left(\frac{\omega b}{c_{t}}\right)-J_{k b+2}\left(\frac{\omega b}{c_{t}}\right)\right], \\
& D_{23}=i\left[Y_{k b-2}\left(\frac{\omega b}{c_{l}}\right)+Y_{k b+2}\left(\frac{\omega b}{c_{l}}\right)\right] \chi^{-1}, \\
& D_{24}=-\left[Y_{k b-2}\left(\frac{\omega b}{c_{t}}\right)-Y_{k b+2}\left(\frac{\omega b}{c_{t}}\right)\right], \\
& D_{31}=\left[J_{k b-2}\left(\frac{\omega a}{c_{l}}\right)+J_{k b+2}\left(\frac{\omega a}{c_{l}}\right)-2(\chi-1) J_{k b}\left(\frac{\omega a}{c_{l}}\right)\right] \frac{a^{2}}{b^{2} \chi}, \\
& D_{32}=i\left[J_{k b-2}\left(\frac{\omega a}{c_{t}}\right)-J_{k b+2}\left(\frac{\omega a}{c_{t}}\right)\right] \frac{a^{2}}{b^{2}}, \\
& D_{42}=-\left[J_{k b-2}\left(\frac{\omega a}{c_{t}}\right)-J_{k b+2}\left(\frac{\omega a}{c_{t}}\right)\right] \frac{a^{2}}{b^{2}}, \\
& D_{33}=\left[Y_{k b-2}\left(\frac{\omega a}{c_{l}}\right)+Y_{k b+2}\left(\frac{\omega a}{c_{l}}\right)-2(\chi-1) Y_{k b}\left(\frac{\omega b}{c_{l}}\right)\right] \frac{a^{2}}{b^{2} \chi}, \\
& D_{34}=i\left[Y_{k b-2}\left(\frac{\omega a}{c_{t}}\right)-Y_{k b+2}\left(\frac{\omega a}{c_{t}}\right)\right] \frac{a^{2}}{b^{2}}, \\
& \left.D_{k b-2}\left(\frac{\omega a}{c_{l}}\right)+Y_{k b+2}\left(\frac{\omega a}{c_{l}}\right)\right] \frac{a^{2}}{b^{2} \chi}, \\
& \left.D_{k b-2}\left(\frac{\omega a}{c_{l}}\right)-J_{k b+2}\left(\frac{\omega a}{c_{l}}\right)\right] \frac{a^{2}}{b^{2} \chi}, \\
& \left.D_{k b-2}\left(\frac{\omega a}{c_{t}}\right)-Y_{k b+2}\left(\frac{\omega a}{c_{t}}\right)\right] \frac{a^{2}}{b^{2}} . \\
& D_{4}
\end{aligned}
$$


The solution of these equations gives the dispersion curves in the circumferential direction.

For the longitudinal wave propagation, three types of wave modes are studied, namely, longitudinal mode, torsional mode, and flexural mode. The particle displacement would be assumed as:

$$
\begin{aligned}
& u_{r}=U(r) \cos (n \theta) e^{i(k z-\omega t)} \\
& u_{\theta}=V(r) \cos (n \theta) e^{i(k z-\omega t)} \\
& u_{z}=W(r) \cos (n \theta) e^{i(k z-\omega t)}
\end{aligned}
$$

$\mathrm{U}, \mathrm{V}, \mathrm{W}$ are the displacement amplitudes composed of Bessel functions. Among them the longitudinal mode particle displacement equations are analyzed below:

$$
\begin{aligned}
& \left(\frac{\partial^{2}}{\partial r^{2}}+\frac{1}{r} \frac{\partial}{\partial r}+\frac{\partial^{2}}{\partial z^{2}}\right) \phi-\frac{1}{c_{l}^{2}} \frac{\partial^{2} \phi}{\partial t^{2}}=0 \\
& \left(\frac{\partial^{2}}{\partial r^{2}}+\frac{1}{r} \frac{\partial}{\partial r}+\frac{\partial^{2}}{\partial z^{2}}\right) \psi-\frac{1}{c_{t}^{2}} \frac{\partial^{2} \psi}{\partial t^{2}}=0
\end{aligned}
$$

The analytical solution for longitudinal mode potential equations is:

$$
\begin{aligned}
& \phi=\Phi(r) e^{i(w t-k z)}=\left(A_{1} J_{0}\left(k_{l} r\right)+A_{2} Y_{0}\left(k_{l} r\right)\right) e^{i(w t-k z)} \\
& \psi=\Psi(r) e^{i(w t-k z)}=\left(A_{3} J_{1}\left(k_{t} r\right)+A_{4} Y_{1}\left(k_{t} r\right)\right) e^{i(w t-k z)} \\
& \phi: \text { scalar potential of longitudal wave } \\
& \psi: \text { vector potential of transverse wave } \\
& k_{t}^{2}=\left(\frac{\omega}{c_{t}^{2}}\right)-k^{2}, k_{l}^{2}=\left(\frac{\omega}{c_{l}^{2}}\right)-k^{2}
\end{aligned}
$$

$\mathrm{J}$ and $\mathrm{Y}$ refer to the first and the second type Bessel functions, respectively.

The general wave equations for three types of transportation modes is expressed as: 


$$
\begin{aligned}
& (\lambda+2 \mu) \frac{\partial \phi}{\partial r}-\frac{2 \mu}{r} \frac{\partial \omega_{z}}{\partial \theta}+2 \mu \frac{\partial \omega_{\theta}}{\partial z}=\rho \frac{\partial^{2} u_{r}}{\partial t^{2}}, \\
& (\lambda+2 \mu) \frac{1}{r} \frac{\partial \phi}{\partial \theta}-2 \mu \frac{\partial \omega_{r}}{\partial z}+2 \mu \frac{\partial \omega_{z}}{\partial r}=\rho \frac{\partial^{2} u_{\theta}}{\partial t^{2}} \\
& (\lambda+2 \mu) \frac{1}{r} \frac{\partial \phi}{\partial z}-\frac{2 \mu}{r} \frac{\partial}{\partial r}\left(r \omega_{\theta}\right)+\frac{2 \mu}{r} \frac{\partial \omega_{r}}{\partial \theta}=\rho \frac{\partial^{2} u_{z}}{\partial t^{2}}
\end{aligned}
$$

Where:

$$
\begin{aligned}
& \varphi=\frac{1}{r} \frac{\partial\left(r u_{r}\right)}{\partial r}+\frac{1}{r} \frac{\partial\left(r u_{\theta}\right)}{\partial \theta}+\frac{\partial u_{z}}{\partial z}, \\
& 2 \omega_{r}=\frac{1}{r} \frac{\partial u_{z}}{\partial \theta}-\frac{\partial u_{\theta}}{\partial z}, \\
& 2 \omega_{\theta}=\frac{\partial u_{r}}{\partial z}-\frac{\partial u_{z}}{\partial r}, \\
& 2 \omega_{z}=\frac{1}{r}\left[\frac{\partial\left(r u_{\theta}\right)}{\partial r}-\frac{\partial u_{r}}{\partial \theta}\right],
\end{aligned}
$$

$u_{i}$ : particle displacement in direction $i$,

$\lambda, \mu:$ Lamb constant, $\rho:$ density.

Introducing scalar potential and vector potential as described at the beginning of this chapter, the wave equation is written as:

$$
\begin{aligned}
& \vec{u}=\nabla \phi+\nabla \times(\vec{\psi}) \\
& \phi: \text { scalar potential of longitudal wave } \\
& \vec{\psi}: \text { vector potential of transverse wave }
\end{aligned}
$$

Expanding this,

$$
\begin{aligned}
& \left(\frac{\partial^{2}}{\partial r^{2}}+\frac{1}{r} \frac{\partial}{\partial r}+\frac{1}{r^{2}} \frac{\partial^{2}}{\partial \theta^{2}}+\frac{\partial^{2}}{\partial z^{2}}\right) \phi+\frac{\omega^{2}}{c_{L}^{2}} \phi=0 \\
& \left(\frac{\partial^{2}}{\partial r^{2}}+\frac{1}{r} \frac{\partial}{\partial r}+\frac{1}{r^{2}} \frac{\partial^{2}}{\partial \theta^{2}}+\frac{\partial^{2}}{\partial z^{2}}\right) \vec{\psi}+\frac{\omega^{2}}{c_{T}^{2}} \vec{\psi}=0
\end{aligned}
$$

By assuming the potential in the forms as below [89],

$$
\begin{aligned}
& \phi=f(r) \cos (n \theta) \cos (\omega t+k z), \\
& \psi_{r}=g_{r}(r) \sin (n \theta) \sin (\omega t+k z), \\
& \psi_{\theta}=g_{\theta}(r) \cos (n \theta) \sin (\omega t+k z), \\
& \psi_{z}=g_{3}(r) \sin (n \theta) \cos (\omega t+k z) .
\end{aligned}
$$


the general solution of Equation (3.29) is:

$$
\begin{aligned}
& f(r)=A_{1} Z_{n}\left(\alpha_{1} r\right)+B_{1} W_{n}\left(\alpha_{1} r\right), \\
& g_{r}(r)=-g_{\theta}(r)=A_{2} Z_{n+1}\left(\beta_{1} r\right)+B_{2} W_{n+1}\left(\beta_{1} r\right), \\
& g_{3}(r)=A_{3} Z_{n}\left(\beta_{1} r\right)+B_{3} W_{n}\left(\beta_{1} r\right) .
\end{aligned}
$$

Where,

$$
\begin{aligned}
& \alpha_{1}=|\alpha|, \alpha^{2}=\omega^{2} / c_{l}^{2}-k^{2}, \\
& \beta_{1}=|\beta|, \beta^{2}=\omega^{2} / c_{t}^{2}-k^{2} .
\end{aligned}
$$

$\mathrm{Z}$ denotes a $\mathrm{J}$ or I Bessel function, and $\mathrm{W}$ denotes a $\mathrm{Y}$ or $\mathrm{K}$ Bessel function. The proper selection of the Bessel function to be used is shown in following.

$$
\begin{aligned}
& \text { For } k<\frac{\omega}{c_{l}} ; \quad J(\alpha r), Y(\alpha r), J(\beta r), Y(\beta r) \\
& \text { For } \frac{\omega}{c_{l}}<k<\frac{\omega}{c_{t}} ; \quad I\left(\alpha_{1} r\right), K\left(\alpha_{1} r\right), J(\beta r), Y(\beta r) ; \\
& \text { For } k>\frac{\omega}{c_{t}} ; \quad I\left(\alpha_{1} r\right), K\left(\alpha_{1} r\right), I\left(\beta_{1} r\right), K\left(\beta_{1} r\right) .
\end{aligned}
$$

The particle displacement and the stress field are derived as [89]:

$$
\begin{aligned}
& u_{r}=\left[f^{\prime}+(n / r) g_{3}+k g_{r}\right] \cos (n \theta) \cos (\omega t+k z), \\
& u_{\theta}=\left[-(n / r) f+k g_{r}-g_{3}^{\prime}\right] \sin (n \theta) \cos (\omega t+k z), \\
& u_{z}=\left[-k f-g_{1}^{\prime}-(n+1) g_{1} / r\right] \cos (n \theta) \sin (\omega t+k z), \\
& \delta_{r r}=\left\{-\lambda\left(\alpha^{2}+k^{2}\right) f+2 \mu\left[f^{\prime \prime}+\frac{n}{r}\left(g_{3}^{\prime}-\frac{g_{3}}{r}\right)+k g_{1}^{\prime}\right]\right\} \cos (n \theta) \cos (\omega t+k z), \\
& \delta_{r \theta}=\mu\left\{-\frac{2 n}{r}\left(f^{\prime}-\frac{f}{r}\right)-2\left(g_{3}^{\prime \prime}-\beta^{2} g_{3}\right)-k\left(\frac{n+1}{r} g_{1}-g_{1}^{\prime}\right)\right\} \sin (n \theta) \cos (\omega t+k z), \\
& \delta_{r z}=\mu\left\{-2 k f^{\prime}-\frac{n}{r}\left[g_{1}^{\prime}+\left(\frac{n+1}{r}-\beta^{2}+k^{2}\right) g_{1}\right]-\frac{n k}{r} g_{3}\right\} \cos (n \theta) \sin (\omega t+k z) .
\end{aligned}
$$

Applying the boundary conditions of free motion on the inner and outer surfaces, $\delta_{r r}=\delta_{r z}=\delta_{r \theta}=0$, at $r=a$, and $r=b$.

The system equation for the coefficients $A_{1}, B_{1}, A_{2}, B_{2}, A_{3}, B_{3}$ is derived as: 


$$
\left[\begin{array}{ccc}
c_{11} & \cdots & c_{16} \\
\vdots & \ddots & \vdots \\
c_{61} & \cdots & c_{66}
\end{array}\right]\left[\begin{array}{c}
A_{1} \\
B_{1} \\
\vdots
\end{array}\right]=0
$$

Since the nontrivial solution of this equation exists only when the determinant of matrix $\mathrm{C}$ is zero, the characteristic equation of a tubular wave system is [89]:

$$
\left|c_{i j}\right|=0, \text { for }(i, j=1 \text { to } 6) \text {. }
$$

Where,

$$
\begin{aligned}
& c_{11}=\left[2 n(n-1)-\left(\beta^{2}-k^{2}\right) a^{2}\right] Z_{n}\left(\alpha_{1} a\right)+2 \lambda_{1} \alpha_{1} a Z_{n+1}\left(\alpha_{1} a\right), \\
& c_{12}=2 k \beta_{1} a^{2} Z_{n}\left(\beta_{1} a\right)-2 k a(n+1) Z_{n+1}\left(\beta_{1} a\right), \\
& c_{13}=-2 n(n-1) Z_{n}\left(\beta_{1} a\right)+2 \lambda_{2} n \beta_{1} a Z_{n+1}\left(\beta_{1} a\right), \\
& c_{14}=\left[2 n(n-1)-\left(\beta^{2}-k^{2}\right) a^{2}\right] W_{n}\left(\alpha_{1} a\right)+2 \alpha_{1} a W_{n+1}\left(\alpha_{1} a\right), \\
& c_{15}=2 \lambda_{2} k \beta_{1} a^{2} W_{n}\left(\beta_{1} a\right)-2 k a(n+1) W_{n+1}\left(\beta_{1} a\right), \\
& c_{16}=-2 n(n-1) W_{n}\left(\beta_{1} a\right)+2 n \beta_{1} a W_{n+1}\left(\beta_{1} a\right), \\
& c_{21}=2 n(n-1) Z_{n}\left(\alpha_{1} a\right)-2 \lambda_{1} \alpha_{1} a Z_{n+1}\left(\alpha_{1} a\right), \\
& c_{22}=-k \beta_{1} a^{2} Z_{n}\left(\beta_{1} a\right)+2 k a(n+1) Z_{n+1}\left(\beta_{1} a\right), \\
& c_{23}=-\left[2 n(n-1)-\beta^{2} a^{2}\right] Z_{n}\left(\beta_{1} a\right)-2 \lambda_{2} \beta_{1} a Z_{n+1}\left(\beta_{1} a\right), \\
& c_{24}=2 n(n-1) W_{n}\left(\alpha_{1} a\right)-2 n \alpha_{1} a W_{n+1}\left(\alpha_{1} a\right), \\
& c_{25}=-\lambda_{2} k \beta_{1} a^{2} W_{n}\left(\beta_{1} a\right)+2 k a(n+1) W_{n+1}\left(\beta_{1} a\right), \\
& c_{26}=-\left[2 n(n-1)-\beta^{2} a^{2}\right] W_{n}\left(\beta_{1} a\right)-2 \beta_{1} a W_{n+1}\left(\beta_{1} a\right), \\
& c_{31}=2 n k \alpha_{1} Z_{n}\left(\alpha_{1} a\right)-2 \lambda_{1} k \alpha_{1} a^{2} Z_{n+1}\left(\alpha_{1} a\right), \\
& c_{32}=-n \beta_{1} a Z_{n}\left(\beta_{1} a\right)-\left(\beta^{2}-k^{2}\right) a^{2} Z_{n+1}\left(\beta_{1} a\right), \\
& c_{33}=-n k a Z_{n}\left(\beta_{1} a\right), \\
& c_{34}=2 n k a W_{n}\left(\alpha_{1} a\right)-2 k \alpha_{1} a^{2} W_{n+1}\left(\alpha_{1} a\right), \\
& c_{35}=\lambda_{2} n \beta_{1} a W_{n}\left(\beta_{1} a\right)-\left(\beta^{2}-k^{2}\right) a^{2} W_{n+1}\left(\beta_{1} a\right), \\
& c_{26}=-n k a W_{n}\left(\beta_{1} a\right) .
\end{aligned}
$$

The other three rows, from $c_{41}$ to $c_{66}$ are obtained from the first three rows by substitution of $b$ with $a$. The parameters $\lambda_{1}$ and $\lambda_{2}$ are defined in Equation (3.37). 
For $k<\frac{\omega}{c_{l}} ; \quad \lambda_{1}=1, \lambda_{2}=1$

For $\frac{\omega}{c_{l}}<k<\frac{\omega}{c_{t}} ; \quad \lambda_{1}=-1, \lambda_{2}=1$

For $k>\frac{\omega}{c_{t}} ; \quad \lambda_{1}=-1, \lambda_{2}=-1$.

Numerical solutions of characteristic equation are then generated for each transportation mode.

For longitudinal and torsional modes, the particle motion is independent of the angular coordinate $\Theta$, i.e. $n=0$, the determinant in Equation (3.35) breaks into the product of two sub-determinants

$$
\left|c_{i j}\right|=D_{1} D_{2}=\left|\begin{array}{llll}
c_{11} & c_{12} & c_{14} & c_{15} \\
c_{31} & c_{32} & c_{34} & c_{35} \\
c_{41} & c_{42} & c_{44} & c_{45} \\
c_{61} & c_{62} & c_{64} & c_{65}
\end{array}\right| \times\left|\begin{array}{cc}
c_{23} & c_{26} \\
c_{53} & c_{56}
\end{array}\right| .
$$

The solution of $\mathrm{D}_{1}=0$ generates the longitudinal modes for a tubular structure, and for $\mathrm{D}_{2}$ $=0$ corresponds to the torsional modes. The numerical solutions for longitudinal modes are illustrated in Figures 3.7. We observe that the group speed of longitudinal wave in a brass tube with $a=5.10 \mathrm{~mm}$ and $b=6.35 \mathrm{~mm}$ is $3720 \mathrm{~m} / \mathrm{s}$ under a frequency of $13 \mathrm{kHz}$, which is one of the input frequencies used in the experiments of this research. This value will be verified later using the experimental data. The numerical solutions of torsional modes for a brass tube are given in Figures 3.8a and 3.8b. The group speed of torsional wave in a brass tube used in the experiments is $2320 \mathrm{~m} / \mathrm{s}$, which can also be verified by experimental results.

For flexural modes, we need to solve the complete set of Equations (3.34) to get solutions under each circumferential order $n$. The numerical solutions for $n=1$ to 3 were calculated and plotted in Figures 3.9 - 3.11 for the experimental brass tubes. Since we are more interested in the frequency band from $13 \mathrm{kHz}$ to $20 \mathrm{kHz}$, all flexural modes in this range are calculated and plotted together in Figure 3.12. Note that there is only one flexural mode for the frequency band $13 \mathrm{kHz}-20 \mathrm{kHz}$. The propagation speed is about $1500 \mathrm{~m} / \mathrm{s}$ for $13 \mathrm{kHz}$. This is verified in the next Section. 


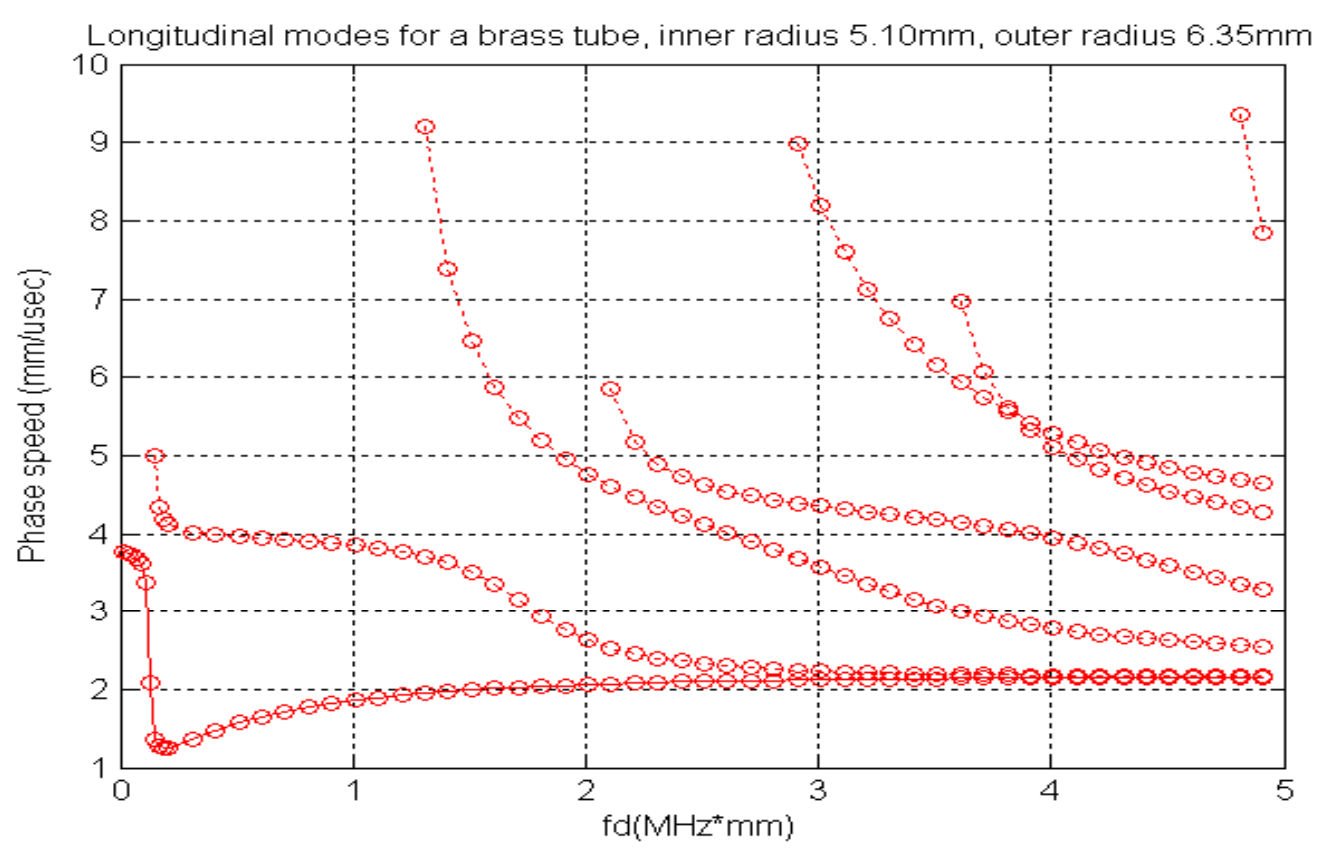

Figure 3.7a. Longitudinal modes in tubular structures, phase speed.

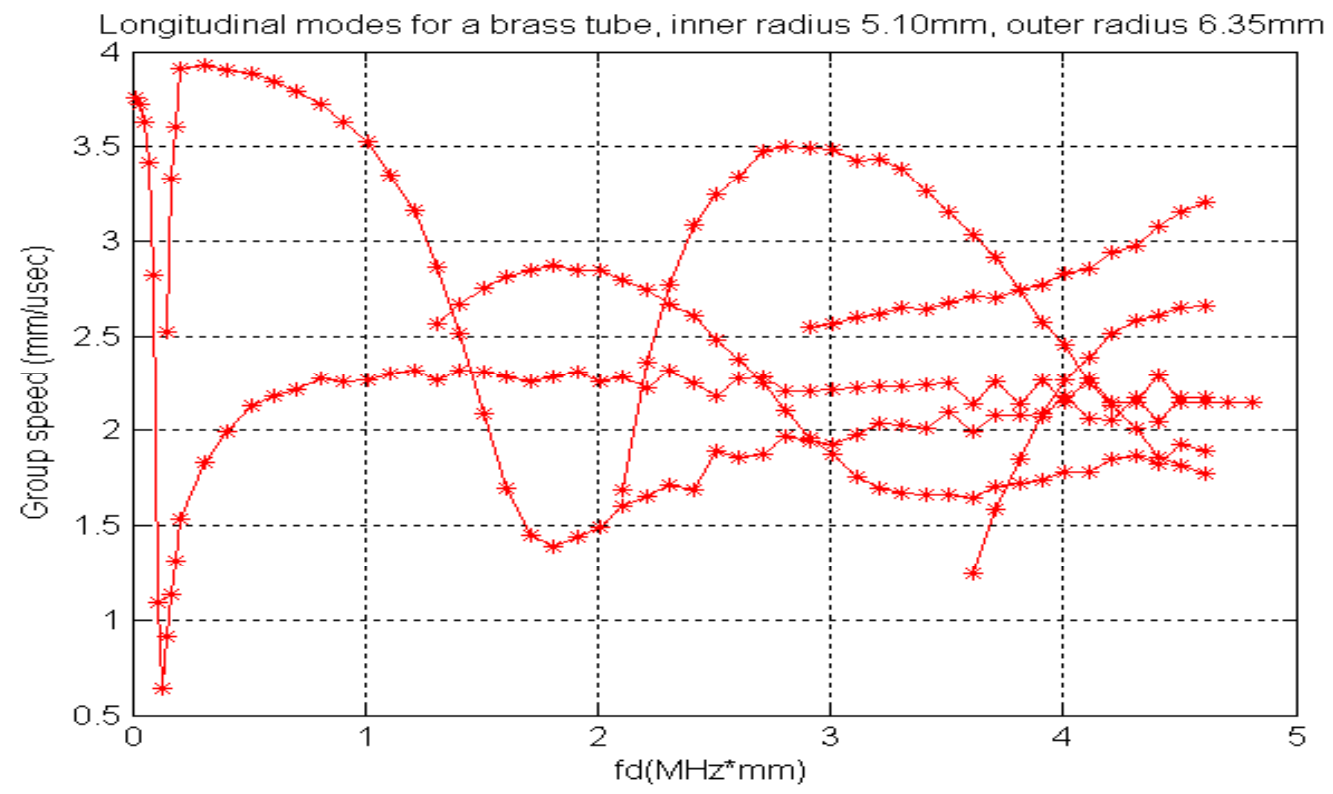

Figure 3.7b. Longitudinal modes in tubular structures, group speed. 


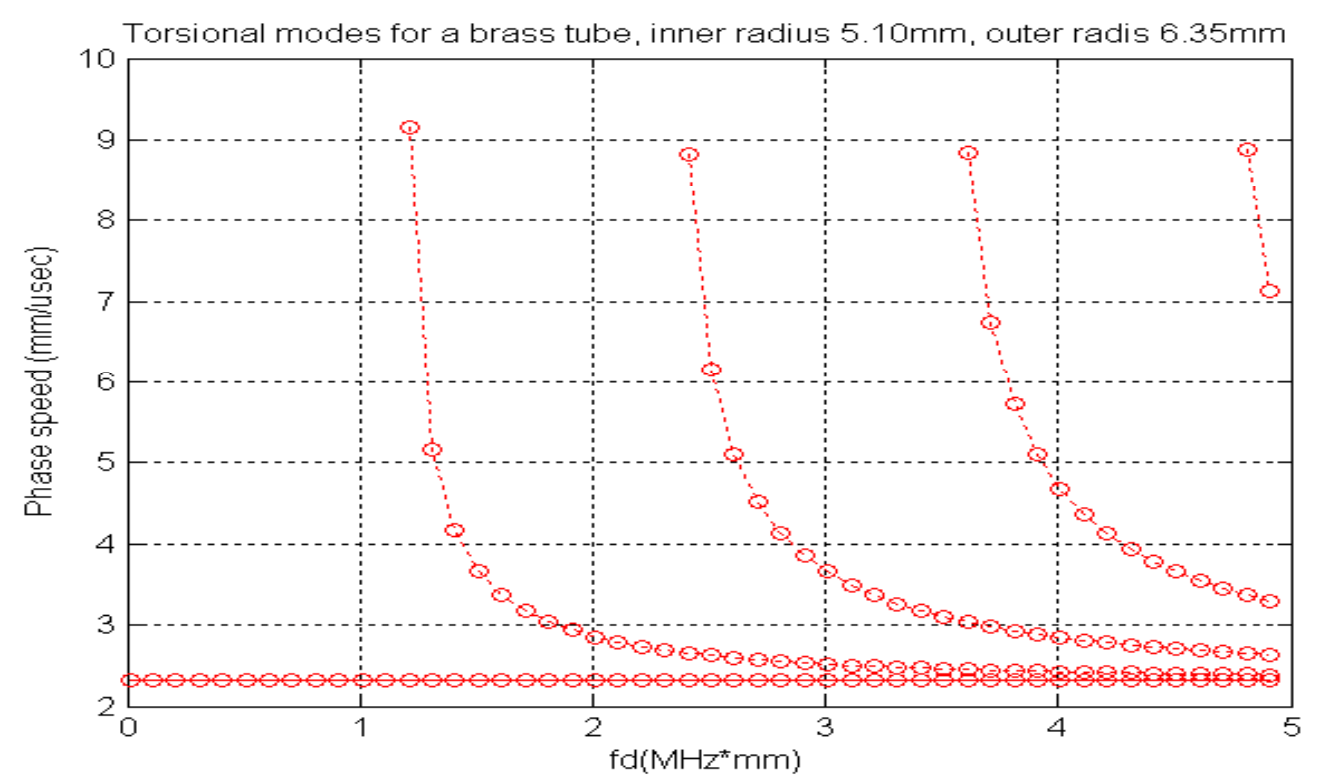

Figure 3.8a. Torsional modes in a brass tube, phase speed.

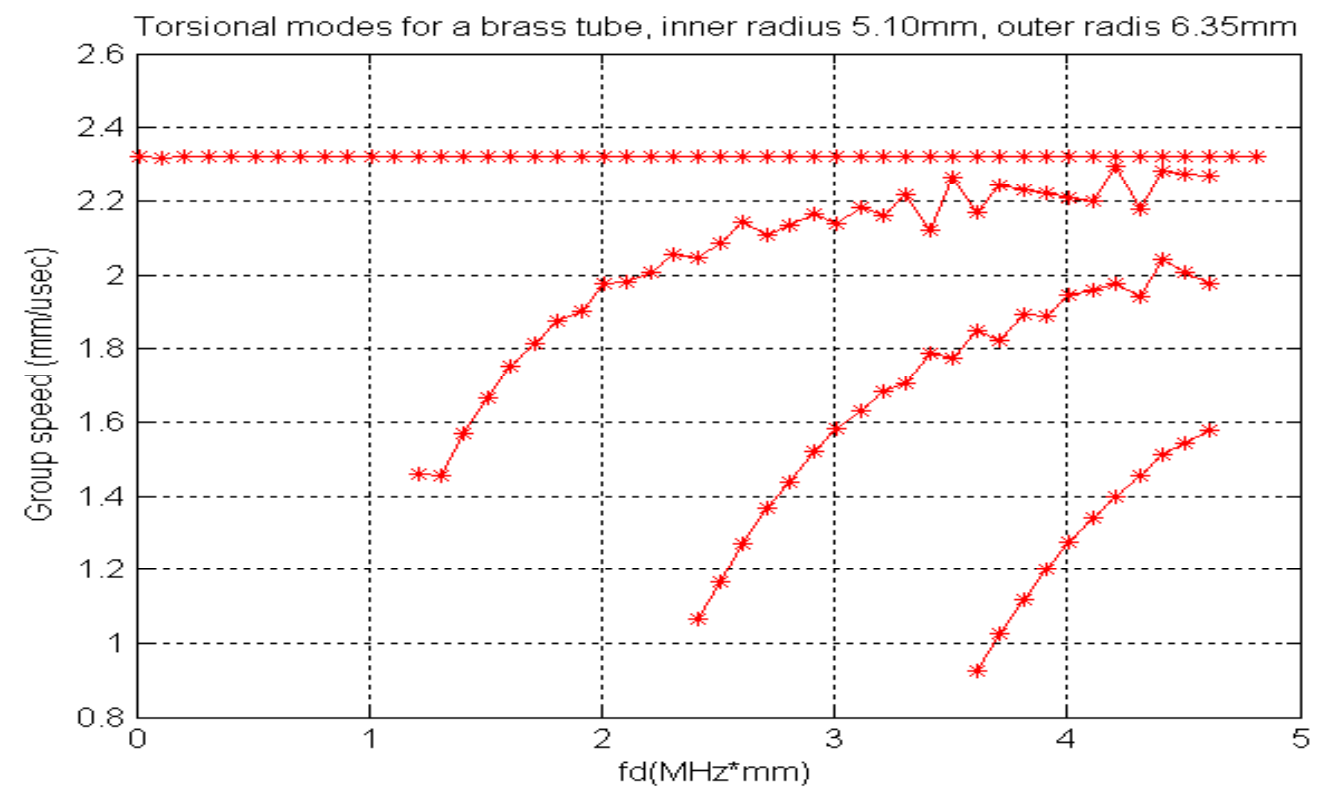

Figure 3.8b. Torsional modes in a brass tube, group speed. 


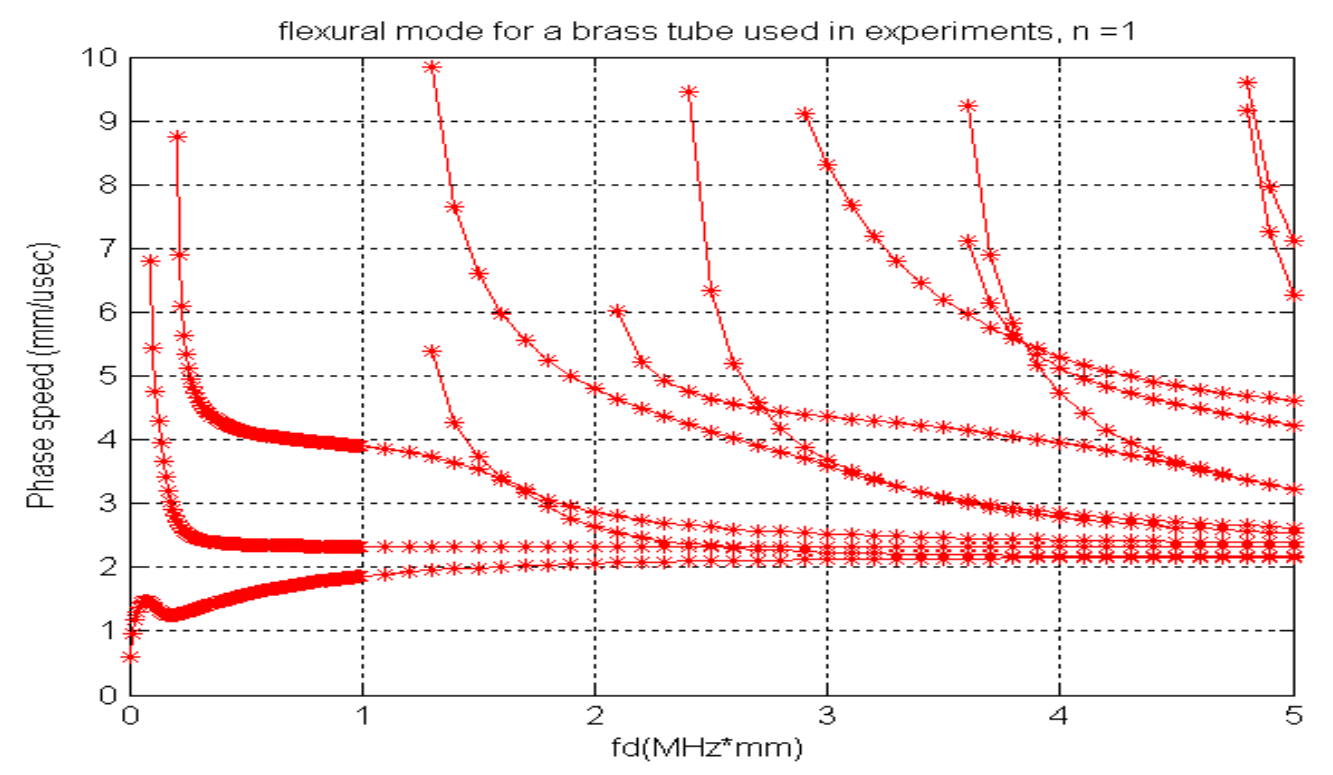

Figure 3.9a. Flexural modes in a brass tube with first circumferential order, phase speed.

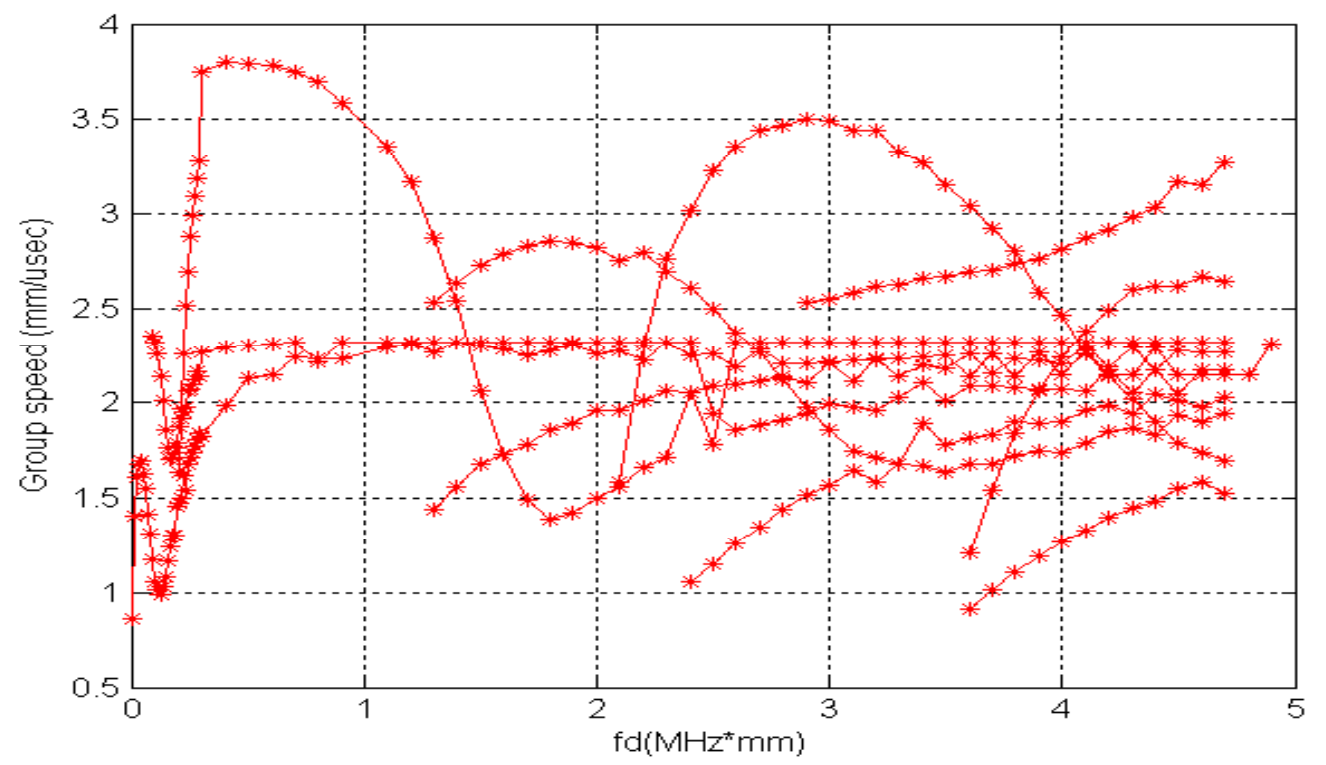

Figure 3.9b. Flexural modes in a brass tube with first circumferential order, group speed. 


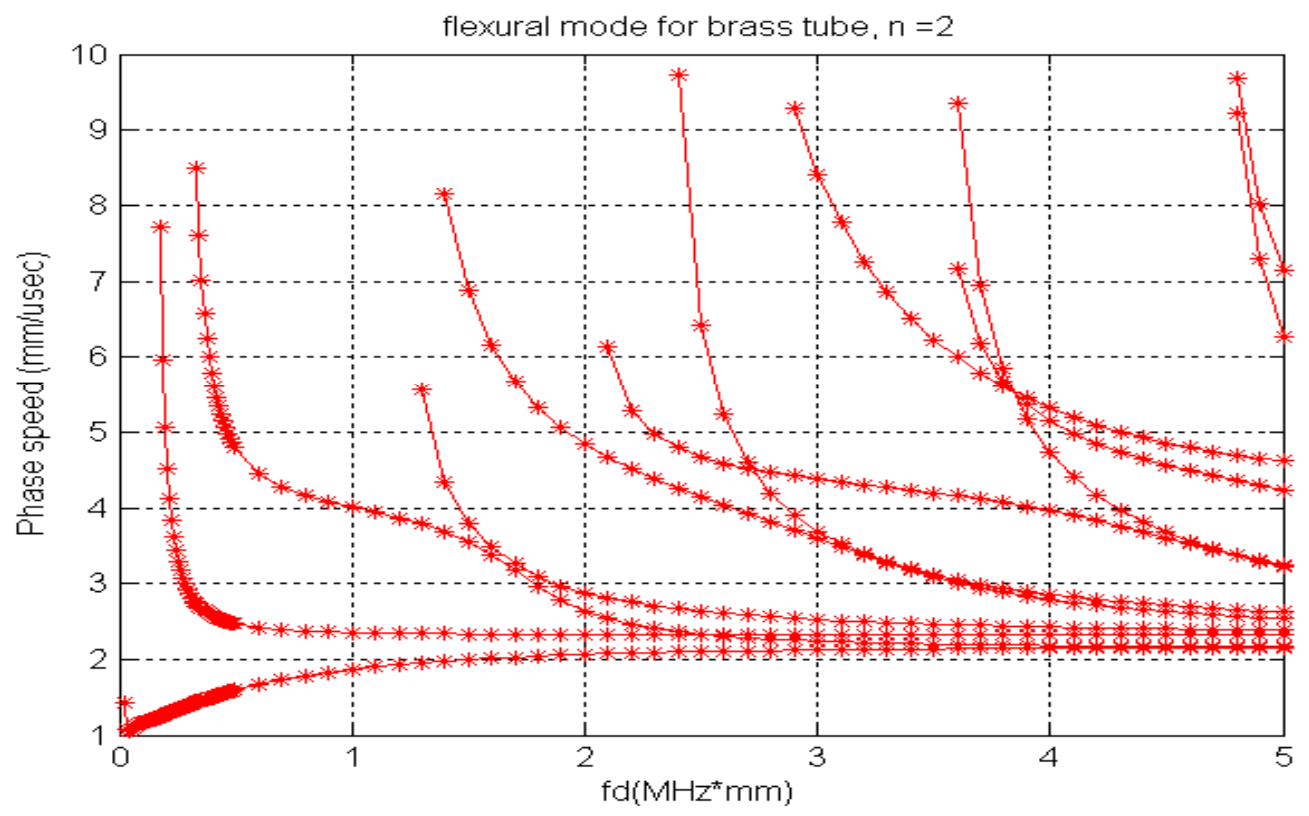

Figure 3.10b. The second circumferential order flexural modes in a brass tube, phase speed.

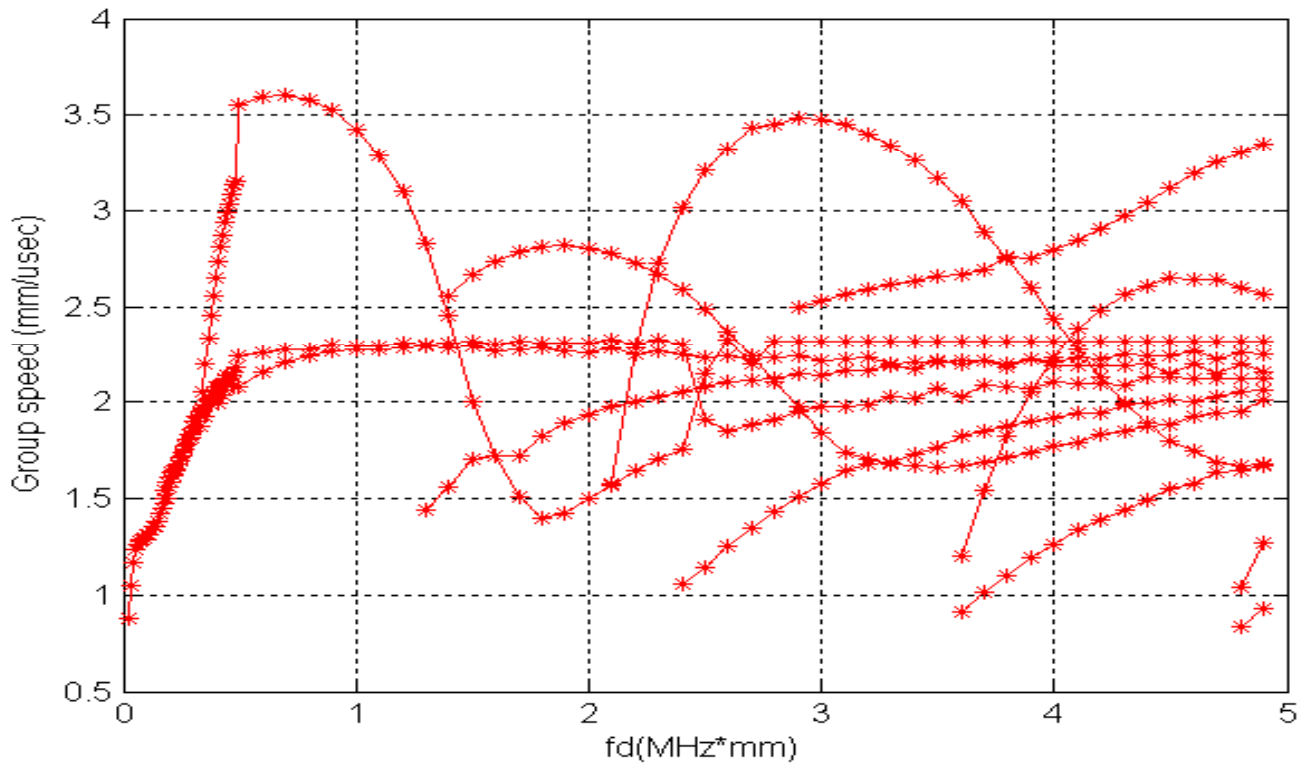

Figure 3.10b. The second circumferential order flexural modes in a brass tube, group speed. 


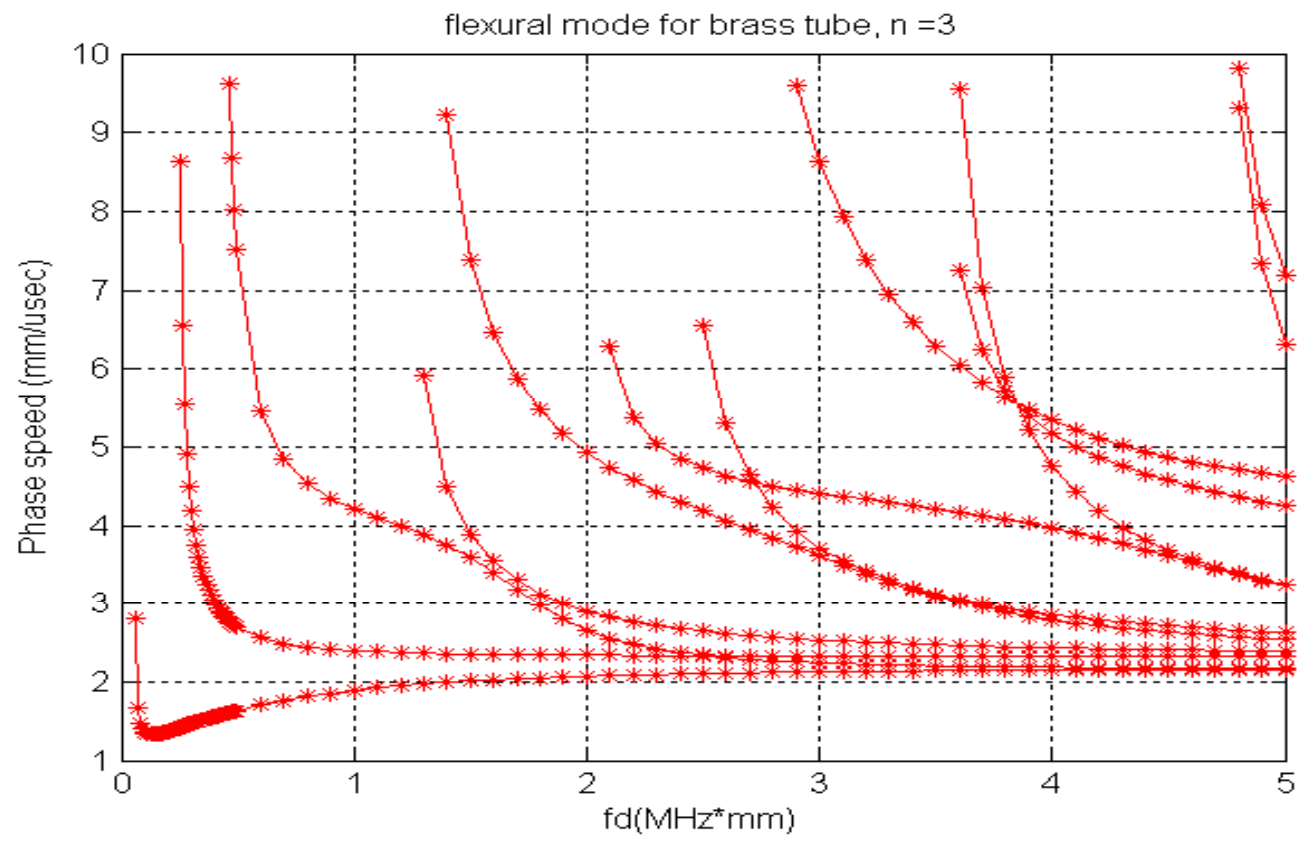

Figure 3.11a. The third circumferential order flexural modes in a brass tube, phase speed.

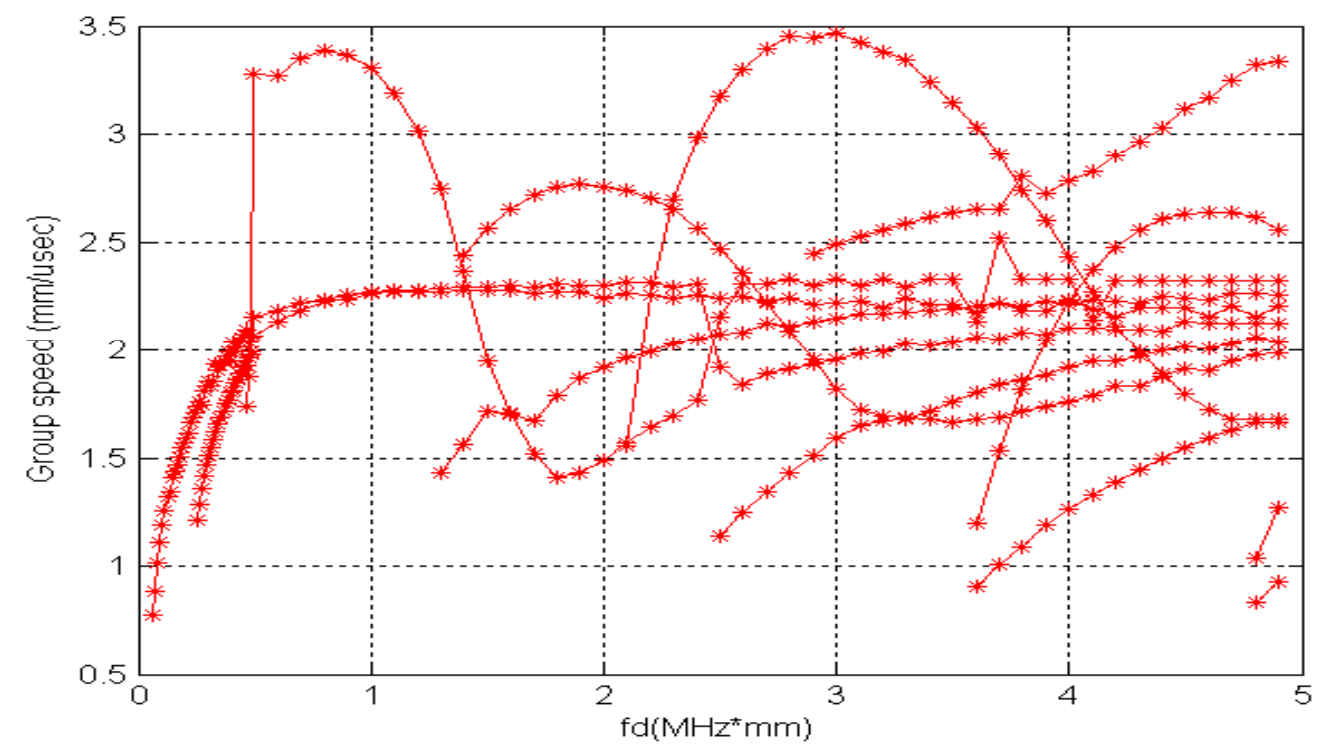

Figure 3.11b. The third circumferential order flexural modes in a brass tube, group speed. 


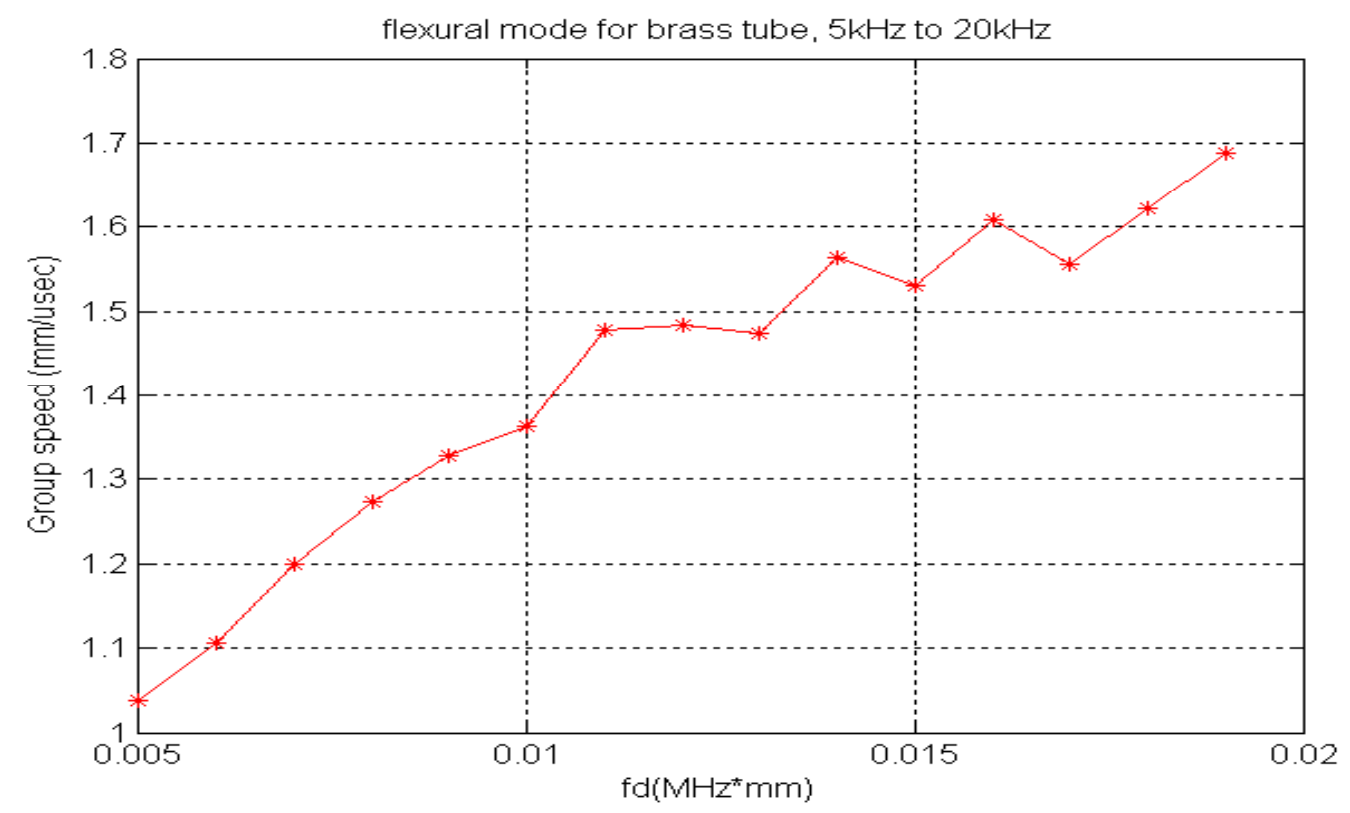

Figure 3.12. The flexural modes in the brass tube used in the experiments, group speed for $5 \mathrm{kHz}$ to $20 \mathrm{kHz}$.

\subsection{Elastic Waves in Metal Structures Submerged in Water}

\subsubsection{Plate specimen immersed in water}

For the plate immersed in the water, we must consider the interaction between plate surface and water. Since only dilatational waves exist in water, the particle displacement equation in water is written as

$$
\begin{aligned}
& \vec{u}_{w}=\nabla \phi_{w} \\
& \phi_{w}: \text { scalar potential of longitudal wave in water } \\
& \vec{u}_{w}: \text { water particle displacementin. }
\end{aligned}
$$

Using the same coordinate system as in Section 3.1 for the aluminum plate, the solution of this equation has the following form: 


$$
\begin{aligned}
& \phi_{w}=E * \sin \left(y \sqrt{k_{w}{ }^{2}-k^{2}}\right) e^{i(k z-\omega t)}+F * \cos \left(y \sqrt{k_{w}^{2}-k^{2}}\right) e^{i(k z-\omega t)}, \\
& k_{w}=\frac{\omega}{c_{w}}, c_{w}: \text { wave speed in water. }
\end{aligned}
$$

The water particle displacements in both directions are derived as:

$$
\begin{aligned}
& u_{x(\text { water })}=0, \\
& u_{y(\text { water })}=\frac{\partial \phi_{w}}{\partial y}=E k_{d} \cos \left(y \sqrt{k_{w}^{2}-k^{2}}\right) e^{i(k z-\omega t)}- \\
& F k_{d} \sin \left(y \sqrt{k_{w}^{2}-k^{2}}\right) e^{i(k z-\omega t)}, \\
& u_{z(\text { water })}=\frac{\partial \phi_{w}}{\partial z}=i k \phi_{w},
\end{aligned}
$$

Where:

$k_{d}=\sqrt{k_{w}^{2}-k^{2}}$

$u_{x, y, z(\text { water })}:$ particle displacement in water.

The normal stress on the waterside is:

$\sigma_{y y(\text { water })}=\lambda_{w}\left(\frac{\partial u_{z}}{\partial z}+\frac{\partial u_{y}}{\partial y}\right)=\lambda_{w}\left(-k^{2}-k_{d}^{2}\right) \phi_{w}$,

Where:

$\lambda_{w}:$ Lame constant of water.

The boundary conditions for this water-plane coupling problem are decided by Equation (3.43).

$$
\begin{aligned}
& \sigma_{y z}=0 \text { at } y= \pm \frac{d}{2}, \\
& \sigma_{y y}=\sigma_{y y(\text { water })} \text { at } y= \pm \frac{d}{2}, \\
& u_{y}=u_{y(\text { water })} \text { at } y= \pm \frac{d}{2}, \\
& d: \text { thickness. }
\end{aligned}
$$

Hence the system equation can be derived, based on Lamb wave equations for the plate, described in Equations (3.8) and (3.9). 
For $y= \pm \frac{d}{2}$

$u_{y}=u_{y(\text { water })}$,

$\Rightarrow$

$-B^{\prime} \sqrt{k_{l}^{2}-k^{2}} \cos \left(y \sqrt{k_{l}^{2}-k^{2}}\right)-i k C^{\prime} \cos \left(y \sqrt{k_{t}^{2}-k^{2}}\right)$

$-i k A^{\prime} \sin \left(y \sqrt{k_{l}^{2}-k^{2}}\right)-D^{\prime} \sqrt{k_{t}^{2}-k^{2}} \sin \left(y \sqrt{k_{t}^{2}-k^{2}}\right)=$

$E k_{d} \cos \left(y \sqrt{k_{w}{ }^{2}-k^{2}}\right) e^{i(k z-\omega t)}-F k_{d} \sin \left(y \sqrt{k_{w}^{2}-k^{2}}\right) e^{i(k z-\omega t)}$;

$\delta_{y z}=0$,

$\Rightarrow$

$\mu\left(-2 i k B^{\prime} \sqrt{k_{l}^{2}-k^{2}} \sin \left(y \sqrt{k_{l}^{2}-k^{2}}\right)+C^{\prime}\left(2 k^{2}-k_{t}^{2}\right) \sin \left(y \sqrt{k_{t}^{2}-k^{2}}\right)\right)$

$\mu\left(-2 i k A^{\prime} \sqrt{k_{l}^{2}-k^{2}} \cos \left(y \sqrt{k_{l}^{2}-k^{2}}\right)+D^{\prime}\left(2 k^{2}-k_{t}^{2}\right) \cos \left(y \sqrt{k_{t}^{2}-k^{2}}\right)\right)=0$;

$\delta_{y y}=\delta_{y y(\text { water })}$,

$\Rightarrow$

$-\lambda k_{l}^{2} B^{\prime} \cos \left(y \sqrt{k_{l}^{2}-k^{2}}\right)-2 \mu\left(\left(k_{l}^{2}-k^{2}\right) B^{\prime} \cos \left(y \sqrt{k_{l}^{2}-k^{2}}\right)\right.$,

$+i k C^{\prime} \sqrt{k_{t}^{2}-k^{2}} \cos \left(y \sqrt{k_{t}^{2}-k^{2}}\right)-\lambda k_{l}^{2} A^{\prime} \sin \left(y \sqrt{k_{l}^{2}-k^{2}}\right)-$

$2 \mu\left(\left(k_{l}^{2}-k^{2}\right) A^{\prime} \sin \left(y \sqrt{k_{l}^{2}-k^{2}}\right)-i k D^{\prime} \sqrt{k_{t}^{2}-k^{2}} \cos \left(y \sqrt{k_{t}^{2}-k^{2}}\right)=\right.$

$\lambda_{w}\left(-k^{2}-k_{d}^{2}\right) \phi_{w}$.

A system characteristic equation can be derived in the following form:

$\left|\begin{array}{llllll|l}c_{11} & c_{12} & c_{13} & c_{14} & c_{15} & c_{16} \\
c_{21} & c_{22} & c_{23} & c_{24} & c_{25} & c_{26} \\
c_{31} & c_{32} & c_{33} & c_{34} & 0 & 0 \\
c_{41} & c_{42} & c_{43} & c_{44} & 0 & 0 \\
c_{51} & c_{52} & c_{53} & c_{54} & c_{55} & c_{56} \\
c_{61} & c_{62} & c_{63} & c_{64} & c_{65} & c_{66}\end{array}\right|$\begin{tabular}{|l}
$B^{\prime}$ \\
$C^{\prime}$ \\
$D^{\prime}$ \\
$E$ \\
$F$
\end{tabular} \mid$=0$

Nontrivial solutions exist only if the determinant of matrix $\mathrm{C}$ equals zero.

In order to consider the energy leakage from plate to the water, an imaginary part is introduced into the wave number $\mathrm{k}=\mathrm{k}_{\mathrm{re}}+\mathrm{ik}_{\mathrm{imag}}$ for the plate immersed in water. The imaginary part corresponds to the energy leakage from plate to the water. The wave propagation term $e^{i(k z-\omega t)}=e^{-k_{\text {imag }} z} e^{i\left(k_{r e} z-\omega t\right)}$ includes the effect of wave attenuation due to 
the effect of water at the boundaries. Therefore, we need to generate complex solutions for this equation.

\subsubsection{Plate structure with water loaded on one side}

For the plate with one side in contact with water and the other side free of traction, the solution of this equation has the following form since one coefficient $\mathrm{E}$ is able to describe the continuous particle displacement in the water because only the particle movement on the interface will be used in deriving the system equations:

$$
\begin{aligned}
& \phi_{w}=E * e^{-i y \sqrt{k_{w}{ }^{2}-k^{2}}} e^{i(k z-\omega t)}, \\
& k_{w}=\frac{\omega}{C_{w}}, c_{w}: \text { wave speed in water. }
\end{aligned}
$$

The water particle displacements in both directions are derived as:

$$
\begin{aligned}
& u_{x(\text { water })}=0, \\
& u_{y(\text { water })}=\frac{\partial \phi_{w}}{\partial y}=-i E k_{d} e^{-i k_{d} y} e^{i(k z-\omega t)}, \\
& u_{z \text { (water) }}=\frac{\partial \phi_{w}}{\partial z}=i k \phi_{w},
\end{aligned}
$$

Where:

$k_{d}=\sqrt{k_{w}^{2}-k^{2}}$

$u_{x, y, z(\text { water })}$ : particle displacement in water.

The normal stress on the waterside is:

$$
\sigma_{y y \text { (water) }}=\lambda_{w}\left(\frac{\partial u_{z}}{\partial z}+\frac{\partial u_{y}}{\partial y}\right)=\lambda_{w}\left(-k^{2}-k_{d}^{2}\right) e^{-i k_{d} y} E e^{i(k z-\omega t)} \text {, }
$$

Where :

$\lambda_{w}$ : Lame constant of water.

The boundary conditions become. 


$$
\begin{aligned}
& \sigma_{y z}=0 \quad \text { at } y= \pm \frac{d}{2}, \\
& \sigma_{y y}=\sigma_{y y(\text { water })} \text { at } y=+\frac{d}{2}, \\
& \sigma_{y y}=0 \text { at } y=-\frac{d}{2}, \\
& u_{y}=u_{y \text { (water) }} \text { at } y=+\frac{d}{2}, \\
& d: \text { thickness. }
\end{aligned}
$$

Hence the system equation can be derived, based on Lamb wave equations for plate, as described in Equations (3.8) and (3.9). 
For $y= \pm \frac{d}{2}$

$$
\begin{aligned}
& \delta_{y z}=0, \\
& \Rightarrow \\
& 2 i k B^{\prime} \sqrt{k_{l}^{2}-k^{2}} \sin \left(y \sqrt{k_{l}^{2}-k^{2}}\right)-C^{\prime}\left(2 k^{2}-k_{t}^{2}\right) \sin \left(y \sqrt{k_{t}^{2}-k^{2}}\right) \\
& 2 i k A^{\prime} \sqrt{k_{l}^{2}-k^{2}} \cos \left(y \sqrt{k_{l}^{2}-k^{2}}\right)-D^{\prime}\left(2 k^{2}-k_{t}^{2}\right) \cos \left(y \sqrt{k_{t}^{2}-k^{2}}\right) \\
& =0
\end{aligned}
$$$$
\text { For } y=\frac{d}{2}
$$$$
\delta_{y y}=\delta_{y y(\text { water })} \text {, }
$$$$
\Rightarrow
$$$$
-\lambda k_{l}^{2} B^{\prime} \cos \left(y \sqrt{k_{l}^{2}-k^{2}}\right)-2 \mu\left(\left(k_{l}^{2}-k^{2}\right) B^{\prime} \cos \left(y \sqrt{k_{l}^{2}-k^{2}}\right),\right.
$$$$
+i k C^{\prime} \sqrt{k_{t}^{2}-k^{2}} \cos \left(y \sqrt{k_{t}^{2}-k^{2}}\right)-\lambda k_{l}^{2} A^{\prime} \sin \left(y \sqrt{k_{l}^{2}-k^{2}}\right)-
$$$$
2 \mu\left(\left(k_{l}^{2}-k^{2}\right) A^{\prime} \sin \left(y \sqrt{k_{l}^{2}-k^{2}}\right)-\right.
$$$$
i k D^{\prime} \sqrt{k_{t}^{2}-k^{2}} \cos \left(y \sqrt{k_{t}^{2}-k^{2}}\right)=\lambda_{w}\left(-k^{2}-k_{d}^{2}\right) e^{-i k_{d} y} E e^{i(k z-\omega t)} \text {; }
$$$$
u_{y}=u_{y(\text { water })} \text {, }
$$$$
\Rightarrow
$$$$
-B^{\prime} \sqrt{k_{l}^{2}-k^{2}} \cos \left(y \sqrt{k_{l}^{2}-k^{2}}\right)-i k C^{\prime} \cos \left(y \sqrt{k_{t}^{2}-k^{2}}\right)
$$$$
-i k A^{\prime} \sin \left(y \sqrt{k_{l}^{2}-k^{2}}\right)-D^{\prime} \sqrt{k_{t}^{2}-k^{2}} \sin \left(y \sqrt{k_{t}^{2}-k^{2}}\right)=
$$$$
-i E k_{d} e^{-i k_{d} y} e^{i(k z-\omega t)} \text {; }
$$

For $y=-\frac{d}{2}$

$$
\begin{aligned}
& \delta_{y y}=0, \\
& \Rightarrow \\
& -\lambda k_{l}^{2} B^{\prime} \cos \left(y \sqrt{k_{l}^{2}-k^{2}}\right)-2 \mu\left(\left(k_{l}^{2}-k^{2}\right) B^{\prime} \cos \left(y \sqrt{k_{l}^{2}-k^{2}}\right),\right. \\
& +i k C^{\prime} \sqrt{k_{t}^{2}-k^{2}} \cos \left(y \sqrt{k_{t}^{2}-k^{2}}\right)-\lambda k_{l}^{2} A^{\prime} \sin \left(y \sqrt{k_{l}^{2}-k^{2}}\right)- \\
& 2 \mu\left(\left(k_{l}^{2}-k^{2}\right) A^{\prime} \sin \left(y \sqrt{k_{l}^{2}-k^{2}}\right)\right. \\
& -i k D^{\prime} \sqrt{k_{t}^{2}-k^{2}} \cos \left(y \sqrt{k_{t}^{2}-k^{2}}\right)=0 .
\end{aligned}
$$

The system characteristic equation are derived in the following form: 


$$
\left|\begin{array}{lllll||l}
C_{11} & c_{12} & c_{13} & c_{14} & 0 & A^{\prime} \\
c_{21} & c_{22} & c_{23} & c_{24} & 0 & B^{\prime} \\
C_{31} & c_{32} & c_{33} & c_{34} & c_{35} & C^{\prime} \\
C_{41} & c_{42} & c_{43} & c_{44} & 0 & 0 \\
c_{51} & c_{52} & c_{53} & c_{54} & c_{55} & D^{\prime} \\
E
\end{array}\right|=0
$$

Nontrivial solutions exist only if the determinant of matrix $\mathrm{C}$ equals zero. Complex solutions of the determinant matrix are the wave numbers of the Lamb wave propagating along the plate.

\subsubsection{Tubular specimen immersed in water}

For the tubular specimen immersed in the water, we may consider the interaction between tubular surface and water in a way similar to that for the plate. The particle displacement equation of water is given by

$$
\begin{aligned}
& \vec{u}_{w}=\nabla \phi_{w} \\
& \phi_{w}: \text { scalar potential of longitudal wave in water } \\
& \vec{u}_{w}: \text { water particle displacement. }
\end{aligned}
$$

Expanding the above equation

$$
\left(\frac{\partial^{2}}{\partial r^{2}}+\frac{1}{r} \frac{\partial}{\partial r}+\frac{1}{r^{2}} \frac{\partial^{2}}{\partial \theta^{2}}+\frac{\partial^{2}}{\partial z^{2}}\right) \phi_{w}+\frac{\omega^{2}}{c_{w}^{2}} \phi_{w}=0
$$

Assume the functional form of the potential in the following form [89],

$$
\phi_{w}=f_{w}(r) \cos (n \theta) \cos (\omega t+k z) .
$$

Using the same coordinate system as in Section 3.2 for the tubular structure, the general solution of this equation has the following form.

$$
\begin{aligned}
& f_{w}(r)=A_{w} Z_{n}\left(\alpha_{w} r\right)+B_{w} W_{n}\left(\alpha_{w} r\right), \\
& \text { Where, } \\
& \alpha_{w}=\left|\alpha_{w r}\right|, \alpha_{w r}{ }^{2}=\omega^{2} / c_{w}^{2}-k^{2} .
\end{aligned}
$$

The water particle displacements in both directions are derived as: 


$$
\begin{aligned}
& u_{r(\text { water })}=\frac{\partial \phi_{w}}{\partial r}=f_{w}^{\prime}(r) \cos (n \theta) \cos (\omega t+k z), \\
& u_{z(\text { water })}=\frac{\partial \phi_{w}}{\partial z}=k f_{w}(r) \cos (n \theta) \cos (\omega t+k z),
\end{aligned}
$$

Where:

$u_{r(\text { water })}:$ particle displacement in water.

The normal stress on the waterside is:

$$
\sigma_{r r(\text { water })}=\lambda_{w}\left(-k^{2}-\alpha_{w}^{2}\right) \phi_{w},
$$

The boundary conditions are written as:

$$
\begin{aligned}
& \sigma_{r z}=0, \quad \sigma_{r \theta}=0, a t \quad r=a, r=b, \\
& \sigma_{r r}=\sigma_{r r(\text { water })} \text { at } \quad r=a, r=b, \\
& u_{r}=u_{r(\text { water })} \text { at } r=a, r=b, \\
& a: \text { inner radius, } \\
& b: \text { outer radius. }
\end{aligned}
$$

Hence the system equation can be derived.

$$
\begin{aligned}
& \text { For } r=a, b \\
& u_{y}=u_{y(\text { water })}, \\
& \Rightarrow \\
& {\left[f^{\prime}+(n / r) g_{3}+k g_{r}\right]=f_{w}^{\prime}(r)} \\
& \delta_{r z}=\delta_{r \theta}=0, \\
& \Rightarrow \\
& \left\{-\frac{2 n}{r}\left(f^{\prime}-\frac{f}{r}\right)-2\left(g_{3}^{\prime \prime}-\beta^{2} g_{3}\right)-k\left(\frac{n+1}{r} g_{1}-g_{1}^{\prime}\right)\right\}=0, \\
& \left\{-2 k f^{\prime}-\frac{n}{r}\left[g_{1}^{\prime}+\left(\frac{n+1}{r}-\beta^{2}+k^{2}\right) g_{1}\right]-\frac{n k}{r} g_{3}\right\}=0 ; \\
& \delta_{r r}=\delta_{r r(w a t e r)}, \\
& \Rightarrow \\
& \left\{-\lambda\left(\alpha^{2}+k^{2}\right) f+2 \mu\left[f^{\prime \prime}+\frac{n}{r}\left(g_{3}^{\prime}-\frac{g_{3}}{r}\right)+k g_{1}^{\prime}\right]\right\}= \\
& \lambda\left(-k^{2}-\alpha_{w}^{2}\right) f_{w} .
\end{aligned}
$$

A system characteristic equation is then derived in the following form: 


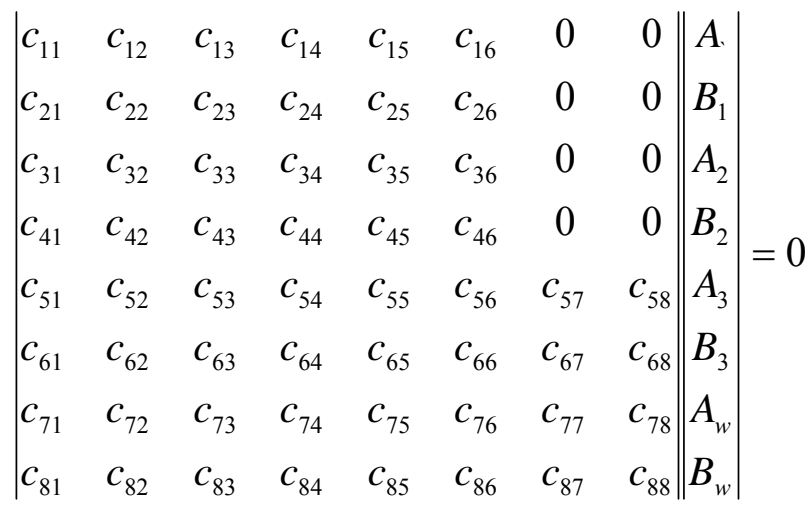

Nontrivial solutions exist only if the determinant of matrix $\mathrm{C}$ equals zero. As mentioned in the previous section, an imaginary part is introduced into the wave number in order to consider the energy leakage from the tube. The imaginary part corresponds to the energy leakage from the tube to the water. Therefore the complex solutions are necessary for the wave propagation along the tube immersed in water.

\subsubsection{Tubular structure with water in contact on the outside}

Since the waves leaving the tube surface are diverging, zero HANKEL function is introduced for the wave propagation in the water because its asymptotic value is zero for $\mathrm{r} \rightarrow \infty$. Using the same coordinate system as in Section 3.2 for the tubular specimen, the general solution of this equation has the following form [30].

$$
\begin{aligned}
& f_{w}(r)=A_{w}\left(J_{0}\left(\alpha_{w} r\right)+i Y_{0}\left(\alpha_{w} r\right)\right)=A_{w} H_{0}\left(\alpha_{w} r\right), \\
& \text { Where, } \\
& \alpha_{w}=\left|\alpha_{w r}\right|, \alpha_{w r}^{2}=\omega^{2} / c_{w}^{2}-k^{2} .
\end{aligned}
$$

The boundary conditions are given by

$$
\begin{aligned}
& \sigma_{r z}=0, \quad \sigma_{r \theta}=0, a t \quad r=a, r=b, \\
& \sigma_{r r}=0, \text { at } r=a, \\
& \sigma_{r r}=\sigma_{r r(\text { water })} \text { at } r=b, \\
& u_{r}=u_{r(\text { water })} \text { at } r=b, \\
& a: \text { inner radius, } \\
& b: \text { outer radius. }
\end{aligned}
$$

The system equation is derived as follows. 
For $r=b$

$$
\begin{aligned}
& u_{y}=u_{y(\text { water })} \\
& \Rightarrow \\
& {\left[f^{\prime}+(n / b) g_{3}+k g_{r}\right]=f_{w}^{\prime}(b) ;}
\end{aligned}
$$

For $r=a, b$

$\delta_{r z}=\delta_{r \theta}=0$,

$\Rightarrow$

$\left\{-\frac{2 n}{r}\left(f^{\prime}-\frac{f}{r}\right)-2\left(g_{3}^{\prime \prime}-\beta^{2} g_{3}\right)-k\left(\frac{n+1}{r} g_{1}-g_{1}^{\prime}\right)\right\}=0$,

$\left\{-2 k f^{\prime}-\frac{n}{r}\left[g_{1}^{\prime}+\left(\frac{n+1}{r}-\beta^{2}+k^{2}\right) g_{1}\right]-\frac{n k}{r} g_{3}\right\}=0$;

For $r=a, \delta_{r r}=0$,

$\Rightarrow$

$\lambda\left(\alpha^{2}+k^{2}\right) f(a)-2 \mu\left[f^{\prime \prime}(a)+\frac{n}{a}\left(g_{3}^{\prime}(a)-\frac{g_{3}(a)}{a}\right)+k g_{1}^{\prime}(a)\right]=0$

For $r=b, \delta_{r r}=\delta_{r r(\text { water })}$,

$$
\begin{aligned}
& \left.\Rightarrow-\lambda\left(\alpha^{2}+k^{2}\right) f(b)+2 \mu\left[f^{\prime \prime}(b)+\frac{n}{r}\left(g_{3}^{\prime}(b)-\frac{g_{3}(b)}{b}\right)+k g_{1}^{\prime}(b)\right]\right\}= \\
& \lambda\left(-k^{2}-\alpha_{w}^{2}\right) f_{w}(b) .
\end{aligned}
$$

A system characteristic equation can be derived in the following form:

$$
\left|\begin{array}{lllllll||}
c_{11} & c_{12} & c_{13} & c_{14} & c_{15} & c_{16} & c_{17} \\
c_{21} & c_{22} & c_{23} & c_{24} & c_{25} & c_{26} & 0 \\
c_{31} & c_{32} & c_{33} & c_{34} & c_{35} & c_{36} & 0 \\
c_{41} & c_{42} & c_{43} & c_{44} & c_{45} & c_{46} & 0 \\
c_{51} & c_{52} & c_{53} & c_{54} & c_{55} & c_{56} & 0 \\
c_{61} & c_{62} & c_{63} & c_{64} & c_{65} & c_{66} & 0 \\
c_{71} & c_{72} & c_{73} & c_{74} & c_{75} & c_{76} & c_{77} \\
B_{1} \\
B_{1} \\
A_{2} \\
B_{2} \\
A_{3} \\
B_{3} \\
A_{w}
\end{array}\right|=0
$$

Nontrivial solutions exist only if the determinant of matrix $\mathrm{C}$ equals zero. The complex solutions are necessary for the wave propagation along the tube with water loaded outside. 


\section{DIGITAL SIGNAL PROCESSING (DSP) TECHNIQUES FOR NON-STATIONARY ACOUSTIC DATA}

Depending on the properties of experimental data and the objectives of this research, several non-stationary signal analysis techniques are proposed for the post signal processing in this study such that more structural properties will be revealed from the experimental data. Some of them are fairly new and have never been used before. As shown in the section above, the Lamb wave speed changes with the transportation mode and frequency, hence neither time nor is frequency analysis alone sufficient to reveal the phenomenon of Lamb wave dispersion. Therefore, time-frequency analysis was very important in this study. An advanced non-linear non-stationary DSP method, HilbertHuang transform (HHT), was introduced and briefly elaborated below as compared with the Wavelet transform. In addition, instantaneous time information plays an important role in structural flaw detection, evaluation, and localization. Two new techniques, called moving windows and zooming windows, are proposed in this section so that more material properties can be revealed from the acquired data. The structural flaw classification using the discrete wavelet transform (DWT) and eigen-face analysis is presented.

\subsection{Hilbert-Huang Transform}

Hilbert transform (HT) is suitable to process non-stationary and narrow band signals. The Hilbert transform is defined as

$$
Y(t)=H(x(t))=\frac{1}{\pi} P \int_{-\infty}^{+\infty} \frac{x(\tau)}{t-\tau} d \tau,
$$

where $\mathrm{P}$ is the Cauchy principal value. From this we can construct an analytical signal

$$
Z(t)=X(t)+i Y(t)=A(t) \exp (i \theta(t))
$$

The instantaneous frequency used in Hilbert transform is written as

$$
\omega(t)=2 \pi f(t)=\frac{d}{d t} \theta(t)
$$


However, the integration of finding $\mathrm{H}[\mathrm{x}(\mathrm{t})]$ is usually complicated, even by using numerical calculation methods. Therefore, Fourier and inverse transformations are often used to calculate $\mathrm{Y}(\mathrm{t})$ as described below.

$$
\begin{aligned}
& x(f)=F F T(x(t)), f=1 \sim 2 N, \\
& z(f)=x(f: 1 \sim N), \text { padding zeros for } N+1 \sim 2 N, \\
& Y(t)=\operatorname{imag}(\operatorname{IFFT}(z(f))) .
\end{aligned}
$$

A time-frequency distribution may be developed using the Hilbert transform. Unfortunately, the application of HT is strictly limited by the properties of $\mathrm{x}(\mathrm{t})$, that is, the signal should be narrow banded around time t. This condition is usually not satisfied by time series collected from practical applications. Suppose that we have a signal $\mathrm{x}(\mathrm{t})=$ $\cos \left(\omega_{1} t\right)+\cos \left(\omega_{2} t\right)$, Hilbert transform will generate an average instantaneous frequency instead of $\omega_{1}$ and $\omega_{2}$ separately. To overcome this problem, Huang et al. [43] proposed an empirical decomposition method to extract intrinsic mode functions from time series such that each intrinsic mode function contains only one simple oscillatory mode (a narrow band at a given time).

An empirical mode decomposition (EMD) algorithm was proposed to generate intrinsic modes in an elegant and simple way, called the sifting process. Three assumptions are made for the EMD of a time series: first, the signal must have at least two extrema - one minimum and one maximum; second, the time interval between the extrema defines the characteristic of the time series; third, if the data were totally devoid of extrema but contained only inflection points, it can be differentiated to reveal the extrema.

Once the extrema are identified, the maxima are connected using a cubic spline and used as the upper envelope. The minima are interpolated as well to form the lower envelope. The upper and lower envelopes should cover all the data points in the time series. The mean of the upper and lower envelopes, $\mathrm{m}_{1}(\mathrm{t})$, is subtracted from the original signal to get the first component $h_{1}(t)$ of this sifting process.

$$
h_{1}(t)=x(t)-m_{1}(t) .
$$

If $h_{1}(t)$ is an intrinsic mode function (IMF), the sifting process stops. Two conditions are used to check $h_{1}(t)$ as an IMF: 1) the number of zero crossings should be equal to the 
number of extrema or differ by at most 1 . In other words, $h_{1}(t)$ should be free of riding waves; 2$) h_{1}(t)$ has the symmetry of upper and lower envelopes with respect to zero.

Otherwise, the sifting process should be repeated to purify the signal $h_{1}(t)$ to an IMF. As a result, $h_{1}(t)$ is sifted to get another first sifted component $h_{11}(t)$.

$$
h_{11}(t)=h_{1}(t)-m_{11}(t),
$$

where $m_{11}(t)$ is the mean of upper and lower envelopes of $h_{1}(t)$. The process continues until $h_{1 k}(t)$ is an IMF. The $h_{1 k}(t)$ is then designated as the first component $c_{1}(t)=h_{1 k}(t)$. In order to stop the sifting process a criterion is defined using a standard deviation.

$$
S D=\sum_{t=1}^{n} \frac{\left|h_{1, k-1}(t)-h_{1, k}(t)\right|^{2}}{h_{1, k-1}^{2}(t)}<\text { threshold. }
$$

The threshold value is usually set between 0.2 and 0.3 [43]. A revised criterion is proposed to accelerate the sifting process.

$$
S D=\frac{\sum_{t=1}^{n}\left|h_{1, k-1}(t)-h_{1, k}(t)\right|^{2}}{\sum_{t=1}^{n} h_{1, k-1}^{2}(t)}<\text { threshold } .
$$

The stopping criterion is designed to keep the resulting IMFs to be physically meaningful. The first component $c_{1}(t)$ contains the finest scale of the signal, or the highest frequency information at each time point. The residual after the first sifting process is

$$
r_{1}(t)=x(t)-c_{1}(t)
$$

Then $r_{1}$ is used to replace the raw signal $x(t)$, and the sifting process continues to generate other IMFs. The sifting process should stop according to the requirement of the physical process. However, there are some general standards, for example, the sum-squared value of the residuals is less than a predefined threshold value or the residual becomes a monotonic function. The residual after sifting out $\mathrm{n}$ components is given by

$$
r_{n}(t)=x(t)-\sum_{i=1}^{n} c_{i}(t)
$$


The resulting IMFs from sifting processes are then ready to be transformed using the Hilbert transform. It is obvious that the resulting empirical components are free from siding (frequencies on either side) waves thus local narrow frequency band is realized. The HHT is adaptive by using the sifting process with the help of cubic interpolations, thus it is a nonlinear transform technique that has great potential applications for complicated non-stationary nonlinear data analysis.

\subsection{Moving Window Method for the Analysis of Time Series of Lamb Waves}

For the finite length tubing, Lamb wave signals collected by piezo-sensors are the combination of several transportation modes that bounce back and forth between the ends or inside discontinuities. The reflection from the boundaries forms local peaks in collected signals in piezo-transducers. However, the wave speed changes along with the frequency for a single mode. Therefore, the synthetic time series usually have narrow peaks at the beginning and wider peaks later. In addition the local peaks are not easy to be found provided enough time is given. The local peaks finally become meaningless as they spread out because too much information is mixed together. In comparison, the first several peaks have clear physical meaning and thus deserve to be separated from the rest for analysis.

A specific type of structural flaw usually interferes with propagating waves in three ways: refraction of part of energy into air or water, reflection of part of the energy back in the form of several modes and transmission of the rest of the energy forward in all modes. The reflection and transmission coefficients are a function of modes as studied by Rose [30], the shape of defect, and the flaw dimension. The experimental studies of reflection/refraction coefficients did not generate constructive results that can be directly applied to NDE techniques. However, one definite correct conclusion is the reflection rate increases nonlinearly with the structural defect size.

In order to study the effect of structural flaws on time series of acoustics in a tube like structure, a moving window with flexible size is implemented to localize the properties of acoustic signals. The principle of this algorithm is demonstrated in Figure 4.1 . 


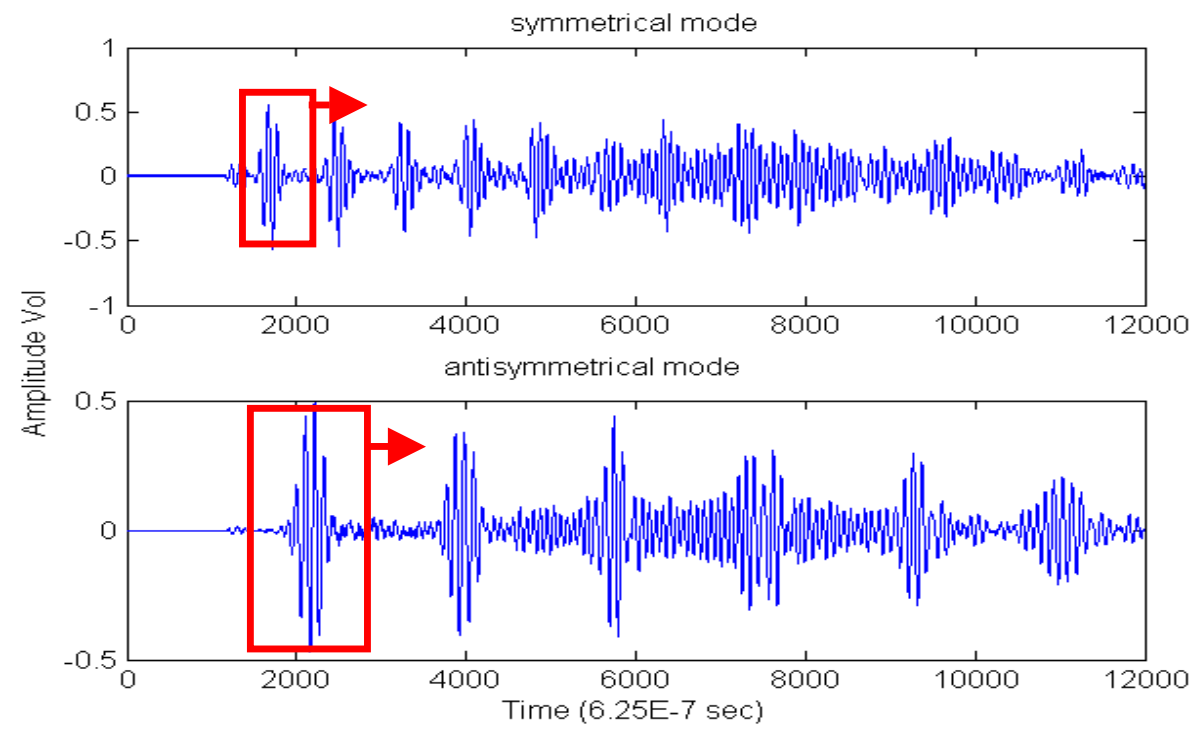

Figure 4.1. Signal localization using moving windows.

The window signals are extracted as long as the middle of the window coincides with a local maximum point, plus it must also be the maximum value among the points falling into the window. The width of the moving window is very critical for good feature extraction. For a large window, suppose its width is larger than two times the distance between two local peaks, the information extracted may not be useful. Too small a window may also create problems, that is, the method may not be sensitive to the existence of structural defects. The selection of an optimal window size depends on the characteristics of the signals, but usually one option is a little bit smaller than twice the distance between two neighboring peaks.

Several properties of windowed signals are introduced in this study and defined as follows:

a. Amplitude in voltage. The energy of local peaks reflects the energy loss in the Lamb wave propagation. The energy loss is due to the refraction of acoustic waves into the air or water from elastic media. A structural discontinuity will 
definitely increase the refraction of acoustic wave into the air and thus decrease the peaks. Part of the energy is reflected back, while the reflection from the defect has certain time of flight and thus also causes the decrease of local acoustic peaks.

b. Spread of the windowed signal. This is defined as

$$
S\left(x_{w}\right)=\frac{\sum_{i=-n}^{n}\left|i^{2} \times x_{w i}\right|}{\sum_{i=-n}^{n}\left|x_{w i}\right|},
$$

Where :

$n:$ half of the window width, $x_{w}$ : windowed signals around a local peak.

c. The variance of windowed signal will increase as a local peak spreads outside its boundaries.

d. Gravity center is defined as:

$$
\begin{gathered}
W_{l}\left(x_{w}\right)=\frac{\sum_{i=-n}^{0}\left|i \times x_{w i}\right|}{\sum_{i=-n}^{0}\left|x_{w i}\right|}, \\
W_{r}\left(x_{w}\right)=\frac{\sum_{i=0}^{n}\left|i \times x_{w i}\right|}{\sum_{i=0}^{n}\left|x_{w i}\right|},
\end{gathered}
$$

Where: $n$ half of the window width, $x_{w}$ : windowed signals around a local peak, $W_{l}$ : gravity center of left half part of the windowed signal, $W_{r}$ : gravity center of right half part of the windowed signal.

The left gravity center and the right gravity center are especially useful in estimating the location of flaw in tubing. Since the first reflection from a structural flaw is located between the first two local peaks selected by a moving window, the right gravity center of the first peak should shift towards the right, and the left gravity center of the second 
peak should shift toward the left, provided the window is large enough to cover the reflected signals. This will be elaborated in the next section about zooming window.

\subsection{Window Zooming Method for the Analysis of Lamb Wave Data}

A zooming window method is proposed to locate the defect in a tube like structure. As will be shown in the next section, the localization of flaw in a tube is much more difficult than in an aluminum plate. Actually the reflected signal in a tube does not appear as an obvious small peak between the local peaks and it is difficult to locate the position of reflection visually. The reason is that the reflection of Lamb waves in a tube spreads between local peaks with certain distribution without an apparent peak, while the distribution depends on the properties of input signals, size of defect, and the shape of defect. The zooming window provides a method for flaw location, and an estimation of the distribution of the reflection signal, as long as enough small step size is given for the window's expansion. The window expansion is illustrated using dashed red lines in Figure 4.2.

For the zooming window method, only the first few local peaks are of interest because of the dispersion of Lamb waves. In other words, the spread of the local peak distorts the reflection after several local peaks, so it is very difficult to distinguish the location information from these local peaks with very large variance.

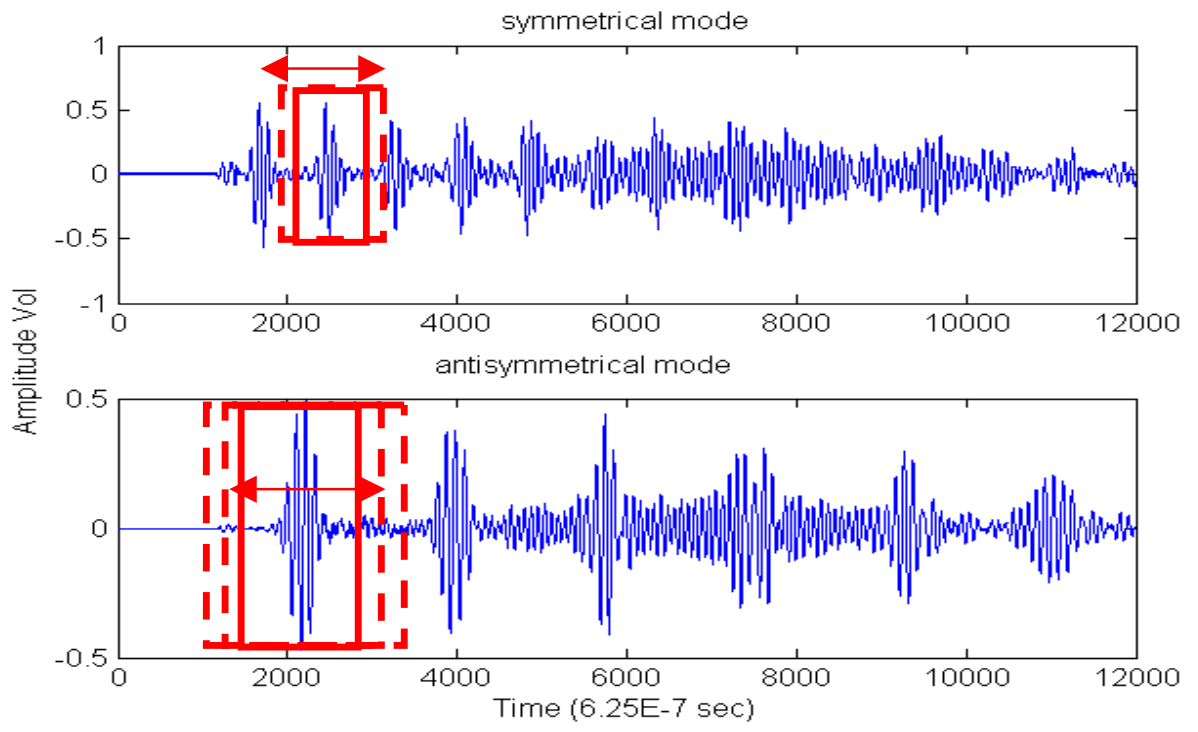

Figure 4.2. Signal localized properties using zooming windows. 


\subsection{Wavelet Transformation and Eigen-face Analysis}

The wavelet transform has been widely used in non-stationary signal analysis for its good properties in capturing both localized time and frequency information. By comparison, STFT (Short-Time Fourier Transform) is especially useful for chirp signals. The limitation mainly comes from the Heisenberg uncertainty principle. The statistical properties of spectrogram from STFT have been used to indicate the structure flaw as described in the 2003 Annual Report. However, the features are not complete for fault evaluation and classification.

A Hanning window modulated sine pulse signal is therefore introduced in order to generate more representative signals for flaw classification. Accordingly, wavelet decomposition is adopted for time-frequency analysis since STFT is not suitable in capturing the abrupt changes in the time series. The continuous wavelet transform is defined as

$$
C W T(\tau, a)=\frac{1}{\sqrt{a}} \int_{-\infty}^{+\infty} f(t) \psi\left(\frac{t-\tau}{a}\right) d t
$$

Where $a$ is a parameter called scale that corresponds to the inverse of the frequency. The wavelet transformation results in a two-dimensional time and frequency image. The coefficients can be viewed as the energy at a point with time $t$ and frequency $f$. However, the direct comparison of WT coefficients is not a good way to detect the structural changes systematically from the data. Based on the wavelet transformation, two dimensional cross correlation analysis provides a different perspective to view the signal change. The cross correlation is calculated as

$$
C_{x y}=\lim \frac{1}{T} \int_{-T / 2}^{T / 2} W C_{x}(t, a) W C_{y}(t+\tau, a) d t .
$$

$\mathrm{WC}_{\mathrm{x}}$ and $\mathrm{WC}_{\mathrm{y}}$ are the coefficients of wavelet transforms of signals $\mathrm{x}$ and $\mathrm{y}$, respectively. The cross correlation reflects the time difference change between signals $\mathrm{x}$ and $\mathrm{y}$. Since 
the structural flaw or thickness change of structure scatters the input waves, cross correlation is a good way to reveal the potential features.

Although the wavelet transform has good properties, it still is limited by the Heisenberg uncertainty principle. The difference is that wavelet analysis decreases the frequency resolution and increases the time domain resolution for high frequency signals, while increases the frequency resolution and sacrifices the time domain resolution for low frequency part of signals as shown in Figure 4.3. This is one of the reasons for introducing the Hilbert-Huang transformation in this research for time-frequency analysis.

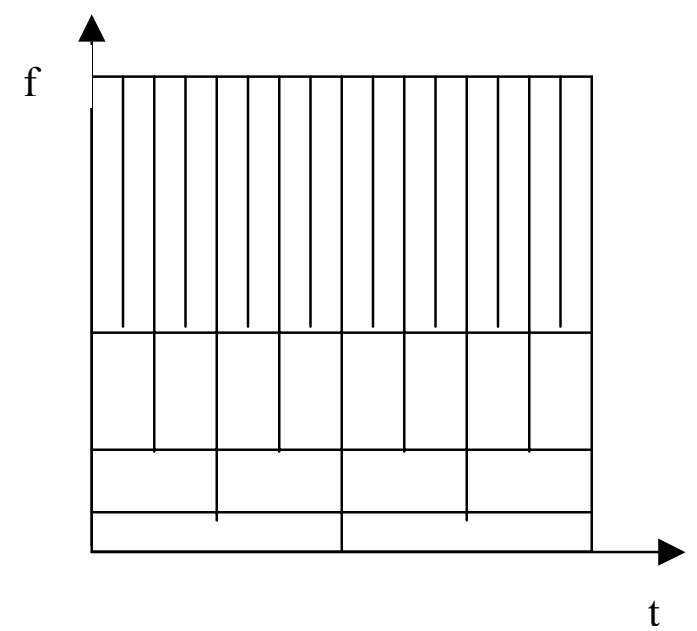

Figure 4.3. Energy distribution of wavelet transformation in the time-frequency domain.

However, WT analysis is still useful in blind classification of structural conditions. The blind classification means the raw wave signals are utilized directly for classification after wavelet decomposition. No other feature extraction techniques are required. The eigen-face method is applied to the time-frequency (T-F) images for classification. The classification process is shown in Figure 4.4. The eigen-face of a set of training images is extracted using the principal component analysis (PCA), where each training image is converted into a vector from a two-dimensional data set. A test T-F image is then projected onto each of the eigen-faces stored in the database. The test image is classified into the one that generates the smallest projection residual. 


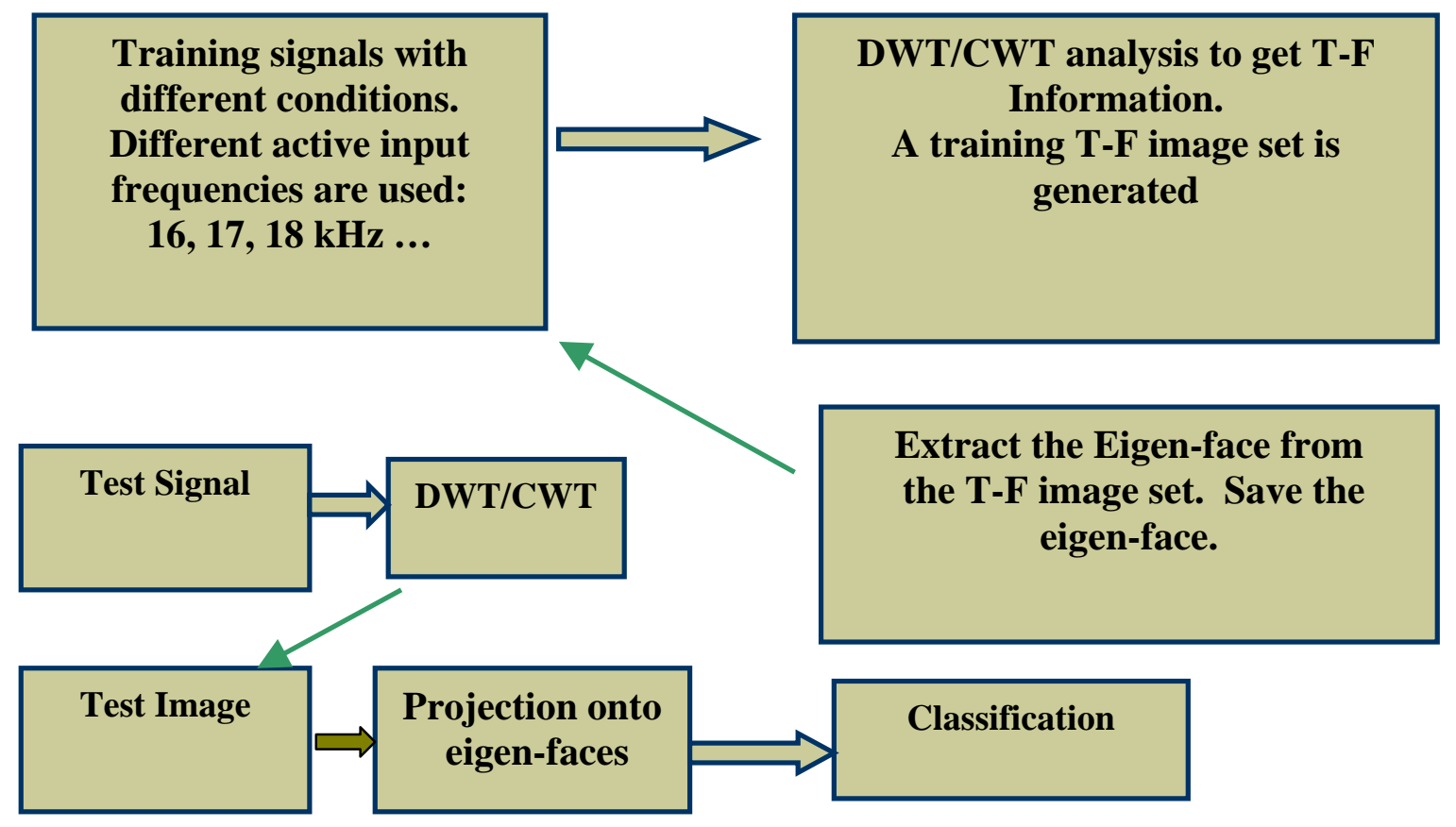

Figure 4.4. DWT/CWT + eigen-face analysis for structural flaw classification.

\subsection{Comparison of Wavelet Transform with HHT}

The wavelet transform method is developed based on the fact that transient signal analysis, such as short time Fourier transform (STFT), could not generate satisfactory resolution with fixed size windows. WT adopts the adjusted window size to improve the time-frequency plot by sacrificing the frequency resolution in the high-frequency band and the time resolution in low-frequency band. However, a dilemma associated with WT is how to explain the decomposed components, since the limited length of the wavelets causes the frequency band under a scale spread around a scaled central frequency as:

$$
F_{a}=\frac{F_{c}}{a \Delta} .
$$


$F_{c}$ is the wavelet central frequency decided by its shape. It is roughly the frequency of a sine wave that best fits the shape of the wavelet, and may be calculated by the peak value of FFT of the wavelet.

$\Delta$ is the sampling interval, and a is the scale.

Hence the result from WT is not real instant frequency component but a series of scaled central frequency approximations. The second reason is that the WT is still limited by uncertainty principle though it tries to decrease its effect. The direct effect is that we need to find the local change in high frequency area because high frequency part has higher time domain resolutions even for the local change happening in low frequency band. There is another difficulty in the implementation of the WT, that is, it is still subjective in selecting the wavelet and evaluating the results using different wavelets.

In comparison, HHT uses an empirical filtering process, which is adaptive instead of having a fixed window. In addition, the definition of Hilbert transform provides the best way to find the instantaneous frequency for a time series. Thus, the HHT is first a nonlinear filtering process while WT is usually linear, and secondly it generates the instantaneous frequency from the definition

$$
\begin{aligned}
& \omega=\frac{d \theta(t)}{d t}, \\
& \theta(t): \text { phase of a time series. }
\end{aligned}
$$

In addition, energy leakage is not avoidable due to the limited length of the wavelets, while the HHT process generates much less leakage. This is illustrated in Figure 4.5, where a sine pulse signal is processed using HHT and WT analyses. We can see that HHT generates the best time-frequency plot in Figure $4.5 \mathrm{~b}$, while the WT + Hilbert transform is better than the pure wavelet transformation. Therefore, the energy leakage in a time-frequency plot from wavelet analysis is caused by two factors: the short length of wavelet, and the methods of instant frequency calculation.

In order to see more advantages of Hilbert transform, another time series defined by $1000 \exp (-2 t) \cos (80 \pi t+1)$, is generated and analyzed using HHT, WT with MORLET wavelet, and WT $+\mathrm{HT}$. 


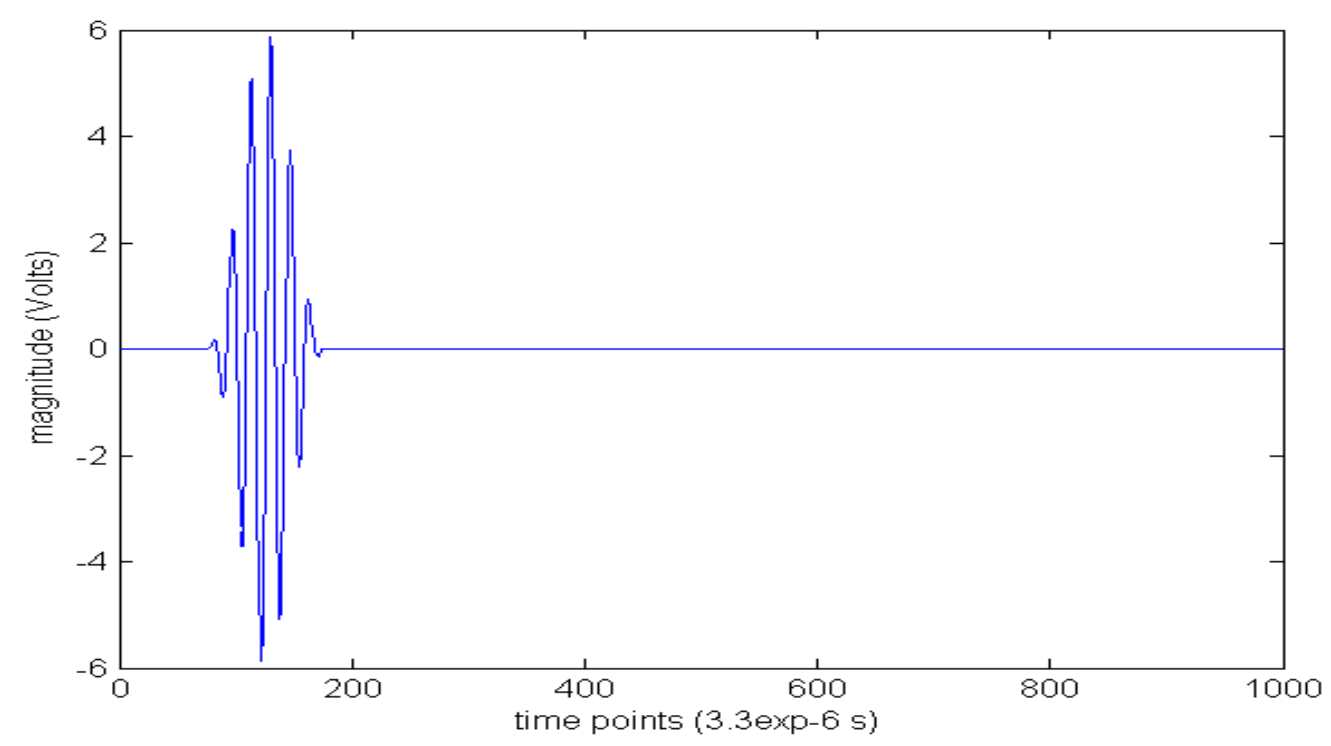

Figure 4.5a. The sine pulse signal adopted in this research.

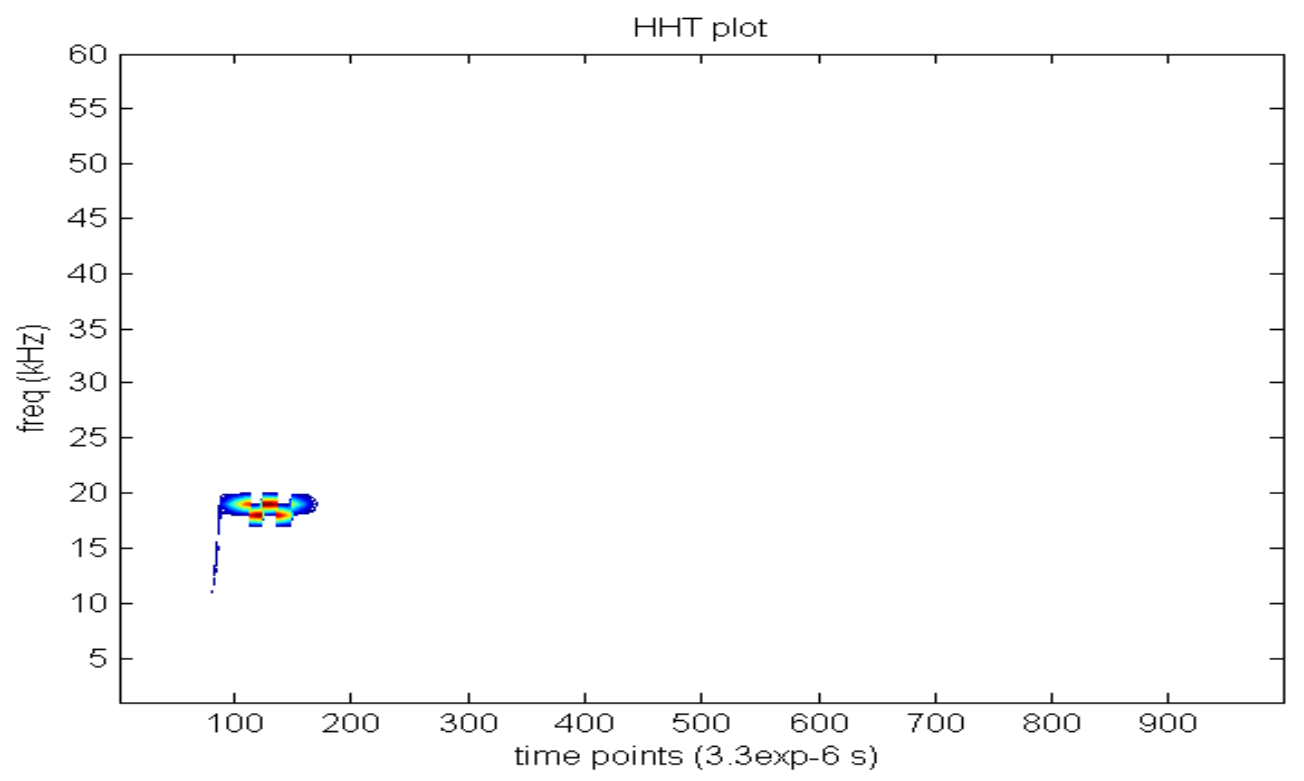

Figure 4.5b. HHT plot of the sine pulse signal, $18 \mathrm{kHz}$. 


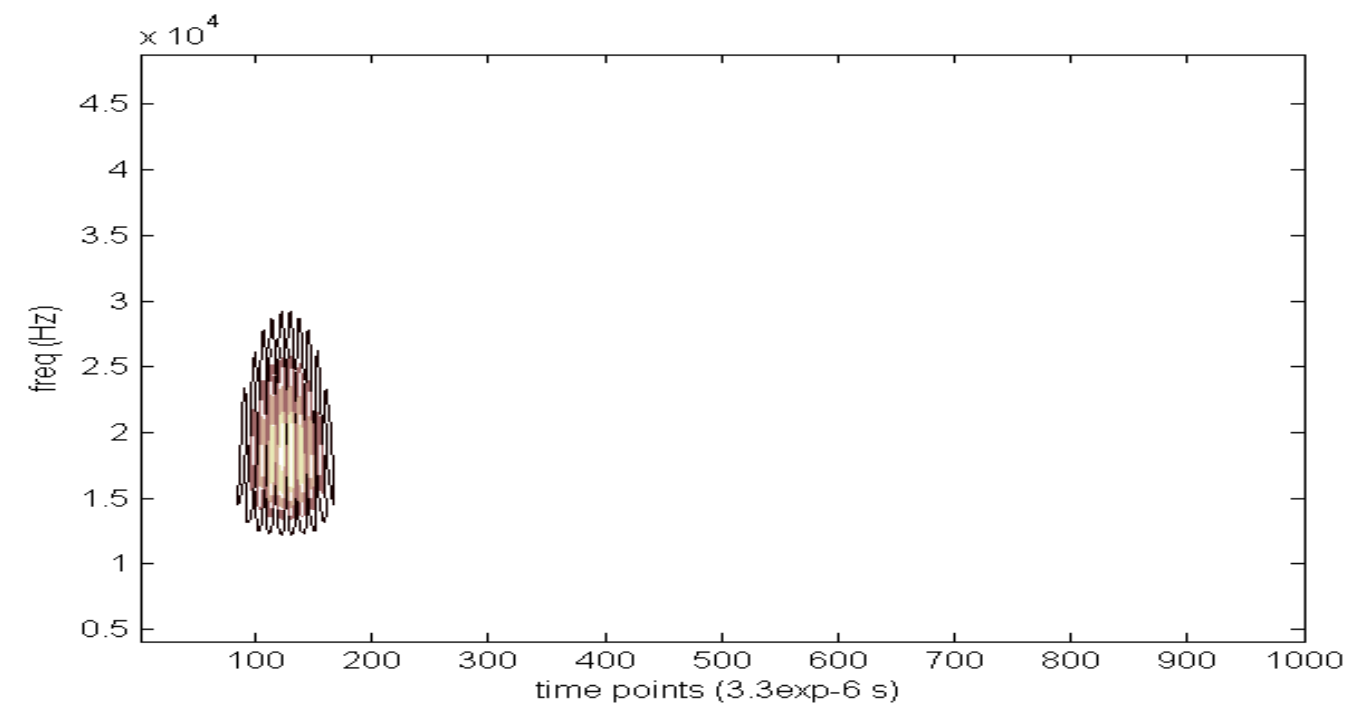

Figure 4.5c. WT plot of the sine pulse signal; a Morlet wavelet was used. The leakage of the energy is obvious compared with $7.5 \mathrm{~b}$.

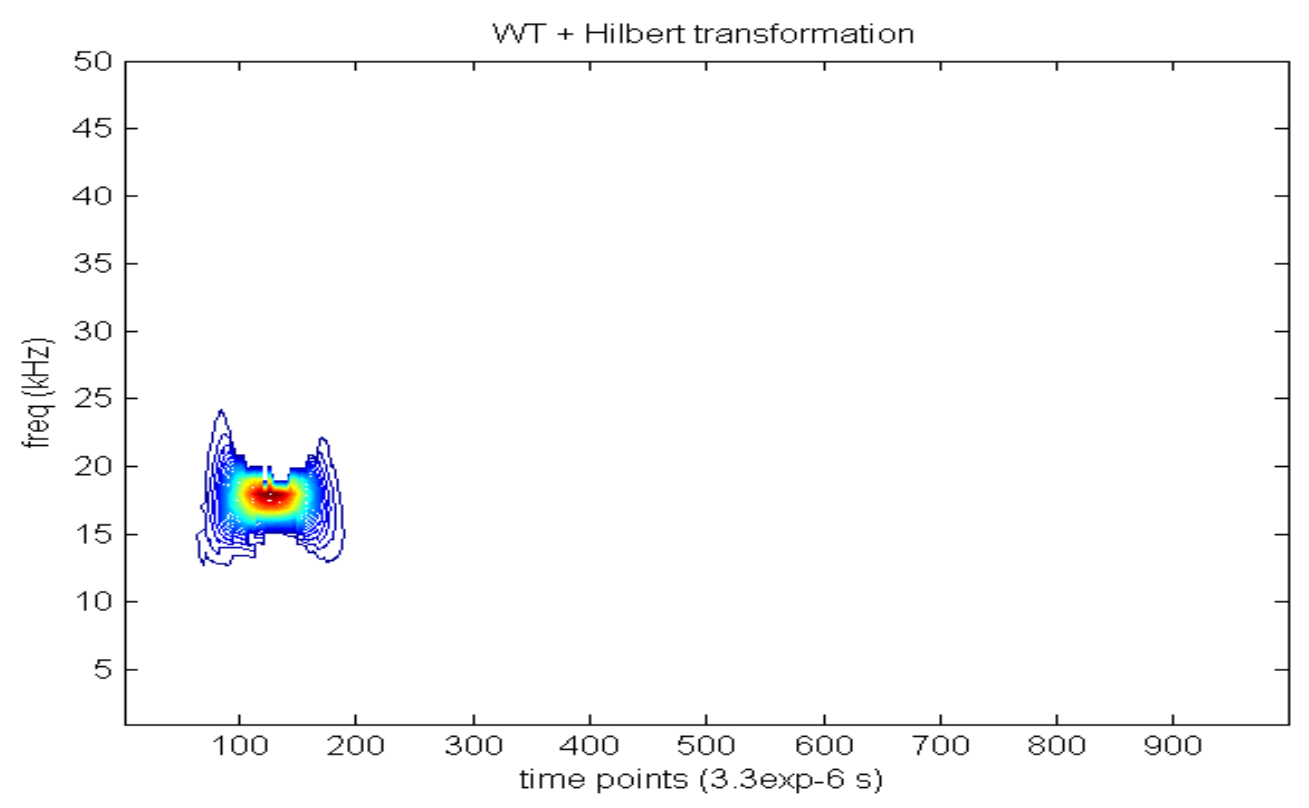

Figure 4.5d. WT + Hilbert transform can improve the concentration of instant frequency. 
The amplitude-modulated signal is plotted in Figure 4.6a. The HHT result is shown in Figure 4.6b, which demonstrates the frequency modulation introduced by amplitude modulation is small and the excellent performance of Hilbert transform in capturing the instantaneous frequency from non-stationary signals. However the wavelet analysis generates a large frequency variation around the central frequency $(20 \mathrm{~Hz})$ as shown in Figure 4.6c, which is caused by the wavelet leakage and the bad definition of instantaneous frequency through scale. The problem of energy leakage cannot be fixed by introducing Hilbert transform after WT decomposition as demonstrated in Figure 4.6d.

A nonlinear time series defined by $100 * \cos (20 * \mathrm{pi} * \mathrm{t}+0.5 * \cos (10 * \mathrm{pi} * \mathrm{t}))$ is generated to test the capability of HHT in processing the signals from nonlinear system. The original signal is plotted in Figure 4.7a. The instantaneous frequency should be $20 \mathrm{pi}-5 * \mathrm{pi} * \cos (10 * \mathrm{pi} * \mathrm{t})$ theoretically from its definition. The Hilbert transform contour plot of the first IMF from EMD gives exactly this instantaneous frequency as being expected as illustrated in Figure 4.7b. While the contour plot of WT results generates a spectrum around $10 \mathrm{~Hz}$ in Figure $4.7 \mathrm{c}$, it is widely distributed and it not able to establish a clear frequency feature from this plot. The process of WT + Hilbert transform illustrated, in Figure 4.7d, is not helpful because the nonlinear properties are mainly related to the signal decomposing process, so the difference comes from the WT process and the EMD nonlinear sifting process.

In summary, the difference between HHT and WT are mainly due to two factors. First, the empirical mode decomposition process is nonlinear and adaptive compared with the linear fitting process used in the WT. Second, the instantaneous frequency calculated by the Hilbert transform may be compared with the correspondence between the frequency and the wavelet scale. Hence, the HHT is highly suitable for the nonlinear and non-stationary signal processing, especially studying the local frequency properties without much energy leakage. 


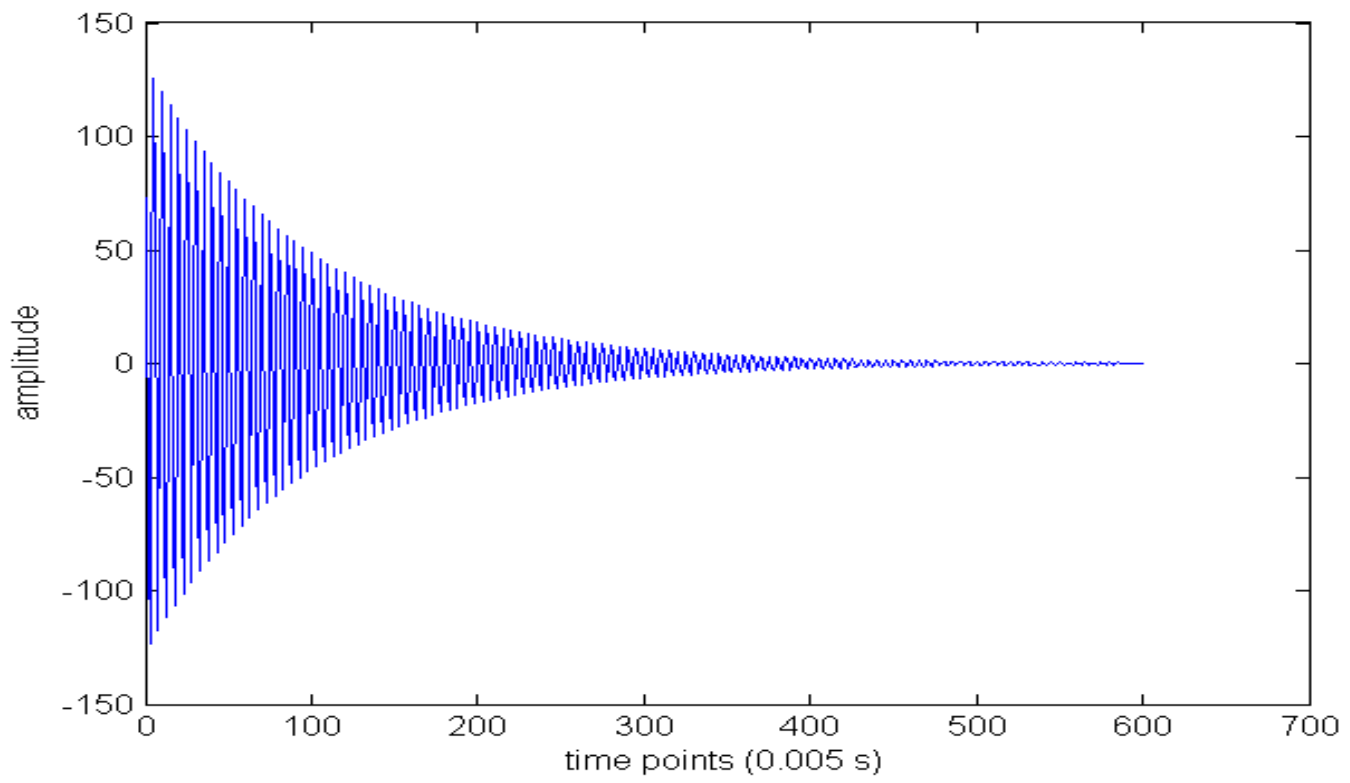

Figure 4.6a. amplitude modulated signals, $1000 * \exp (-2 * \mathrm{t}) * \cos \left(80 * \mathrm{pi}{ }^{*} \mathrm{t}+1\right)$.

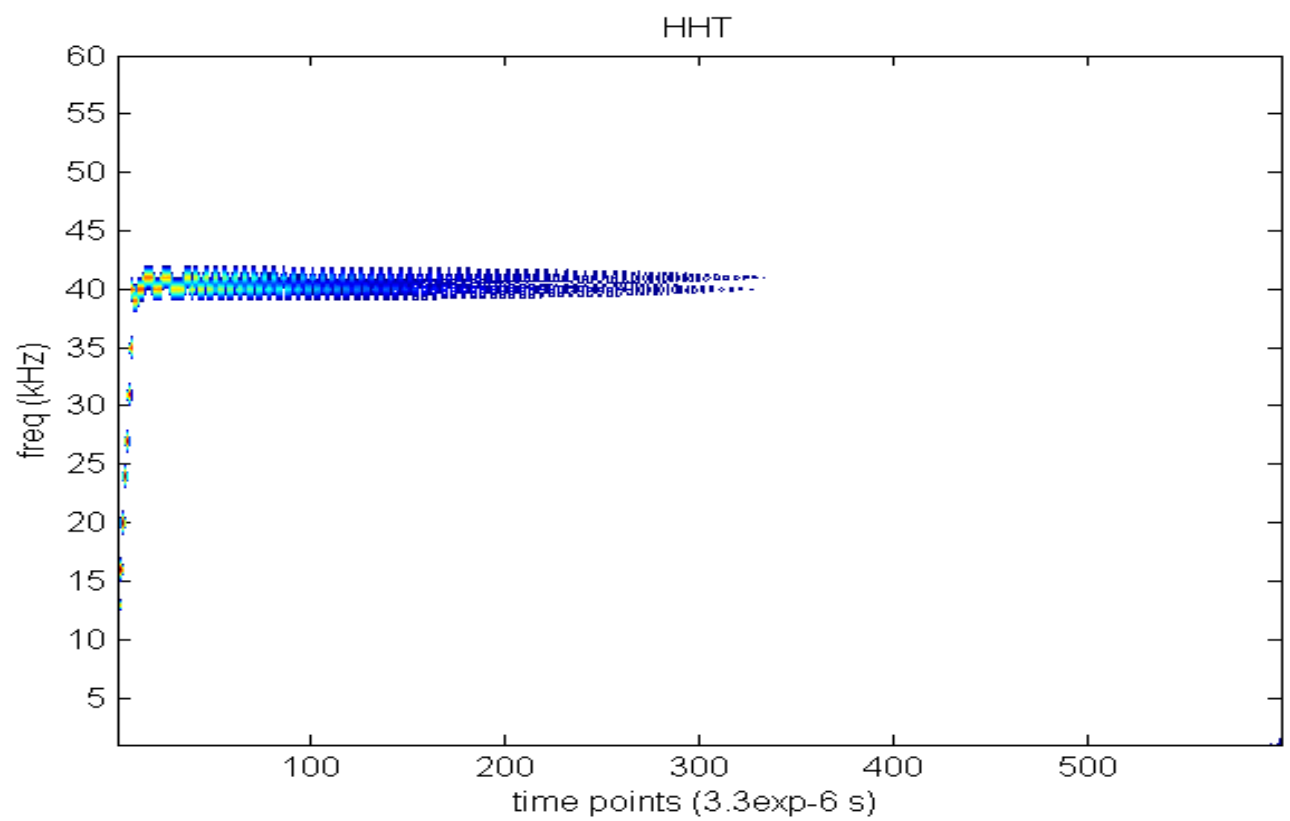

Figure 4.6b. HHT of the amplitude modulated signals illustrates frequency modulation introduced by amplitude modulation is small. 


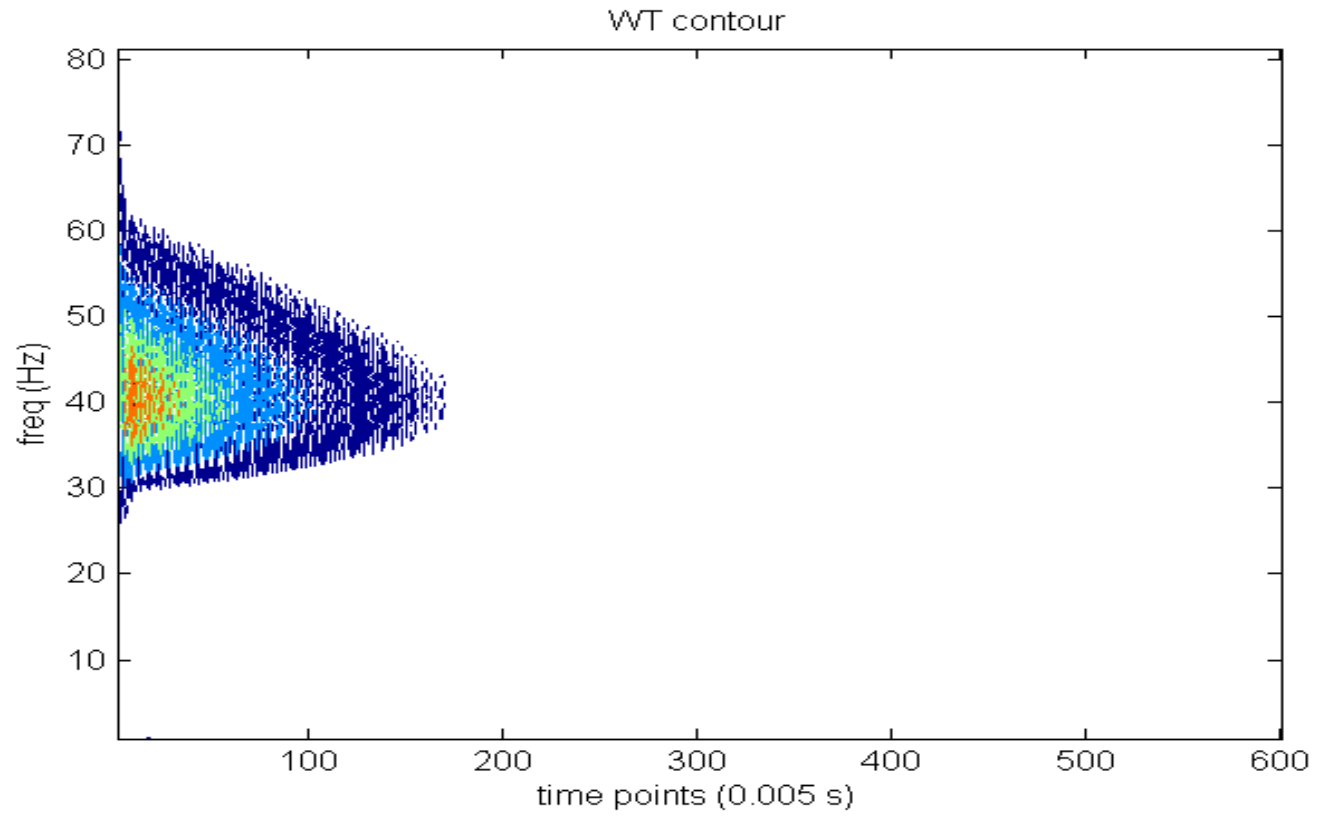

Figure 4.6c. WT of amplitude-modulated signals.

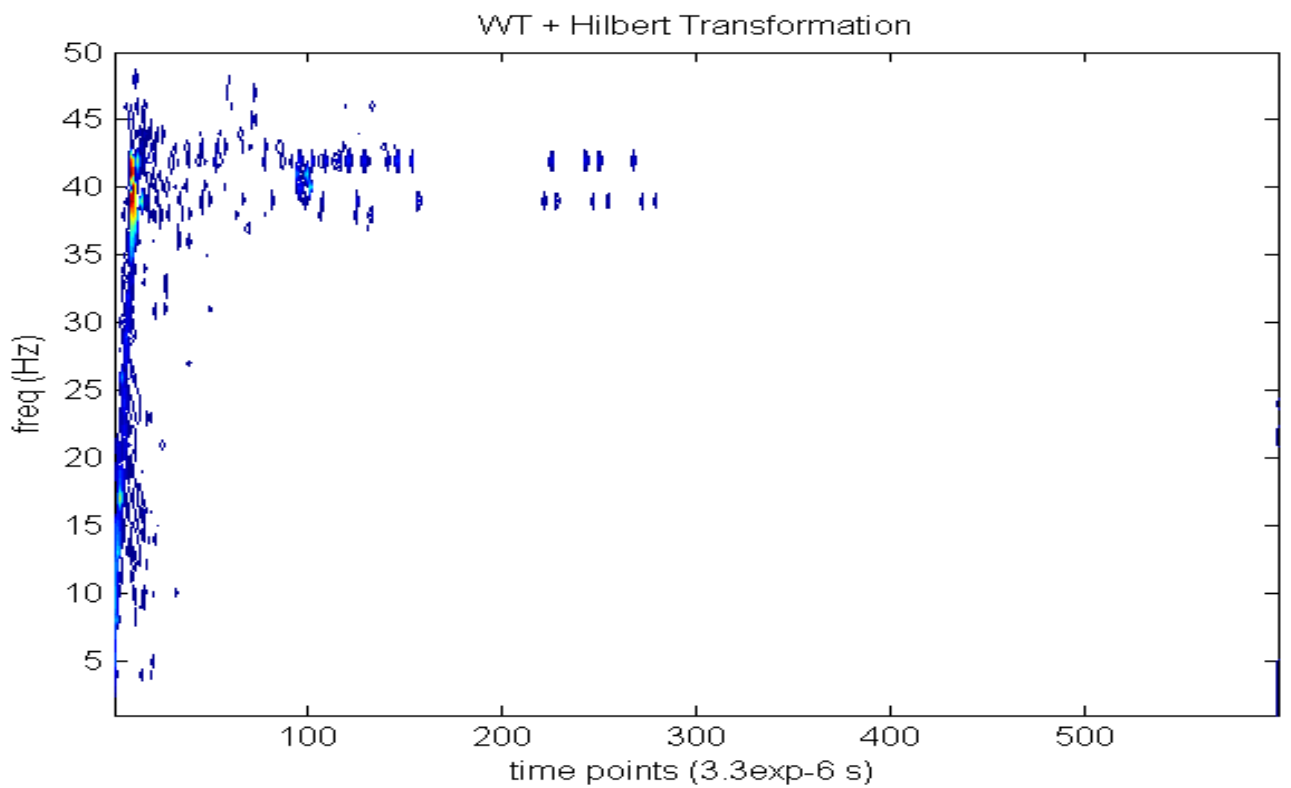

Figure 4.6d. WT + Hilbert transform for amplitude modulated signals. 


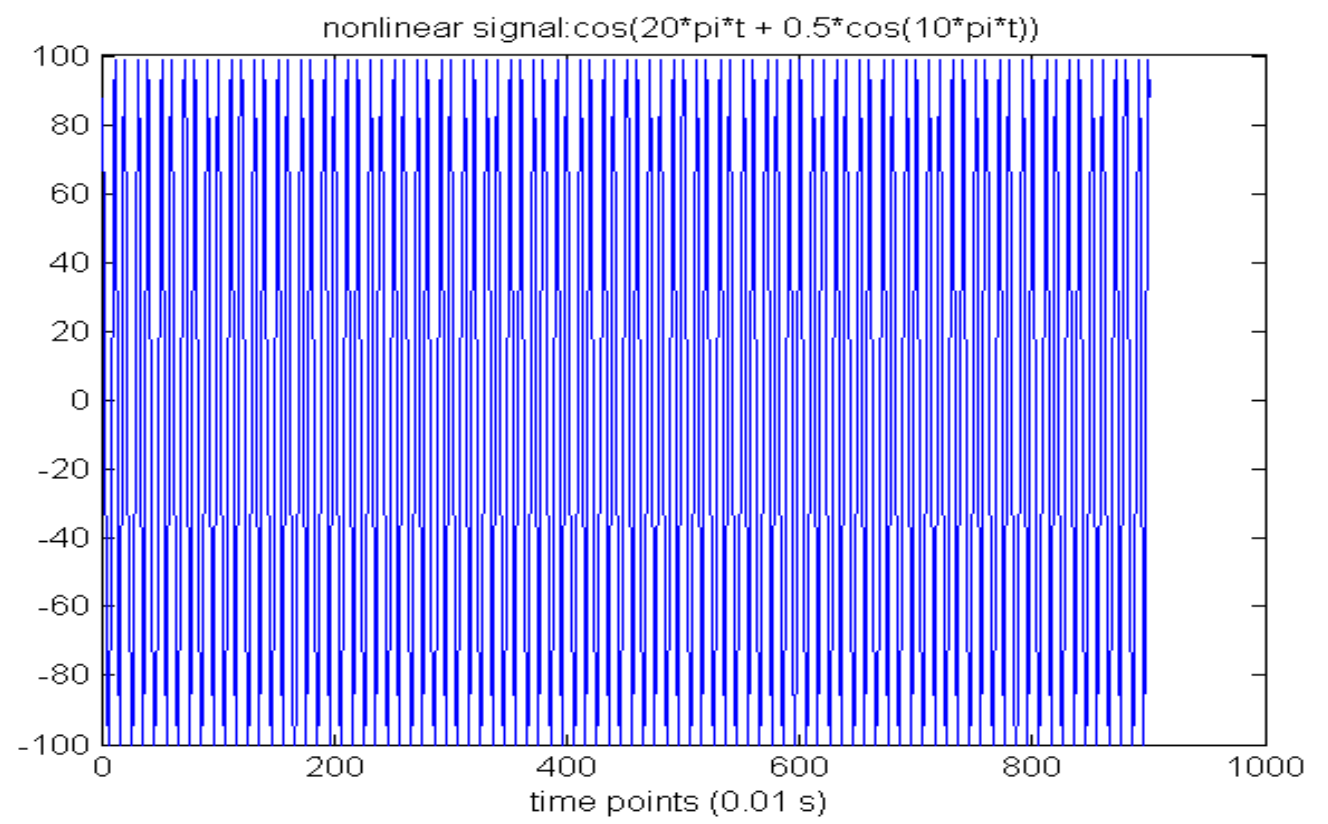

Figure 4.7a. Nonlinear signal defined by $100 * \cos (20 * \mathrm{pi} * \mathrm{t}+0.5 * \cos (10 * \mathrm{pi} * \mathrm{t}))$.

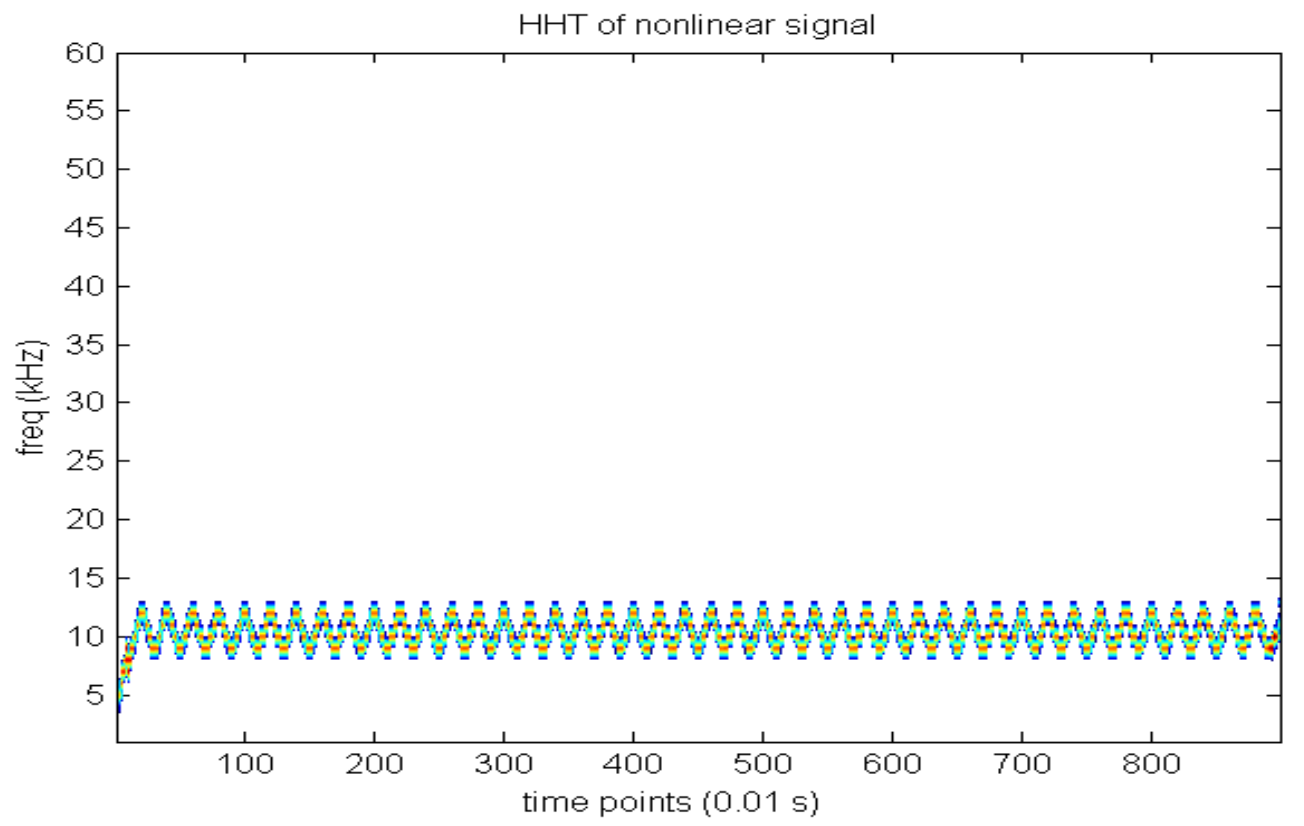

Figure 4.7b, HHT of the nonlinear signal. 


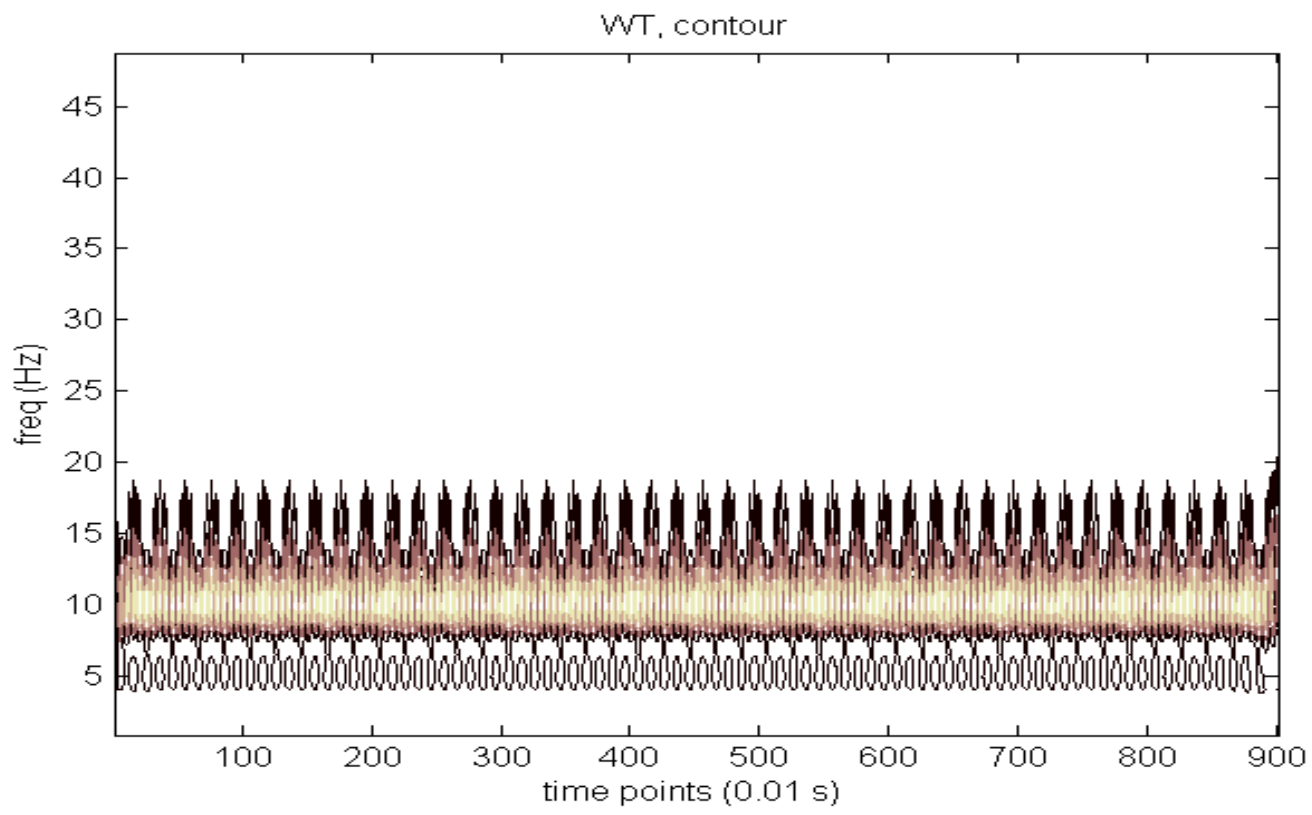

Figure $4.7 \mathrm{c}$. WT of the nonlinear signal.

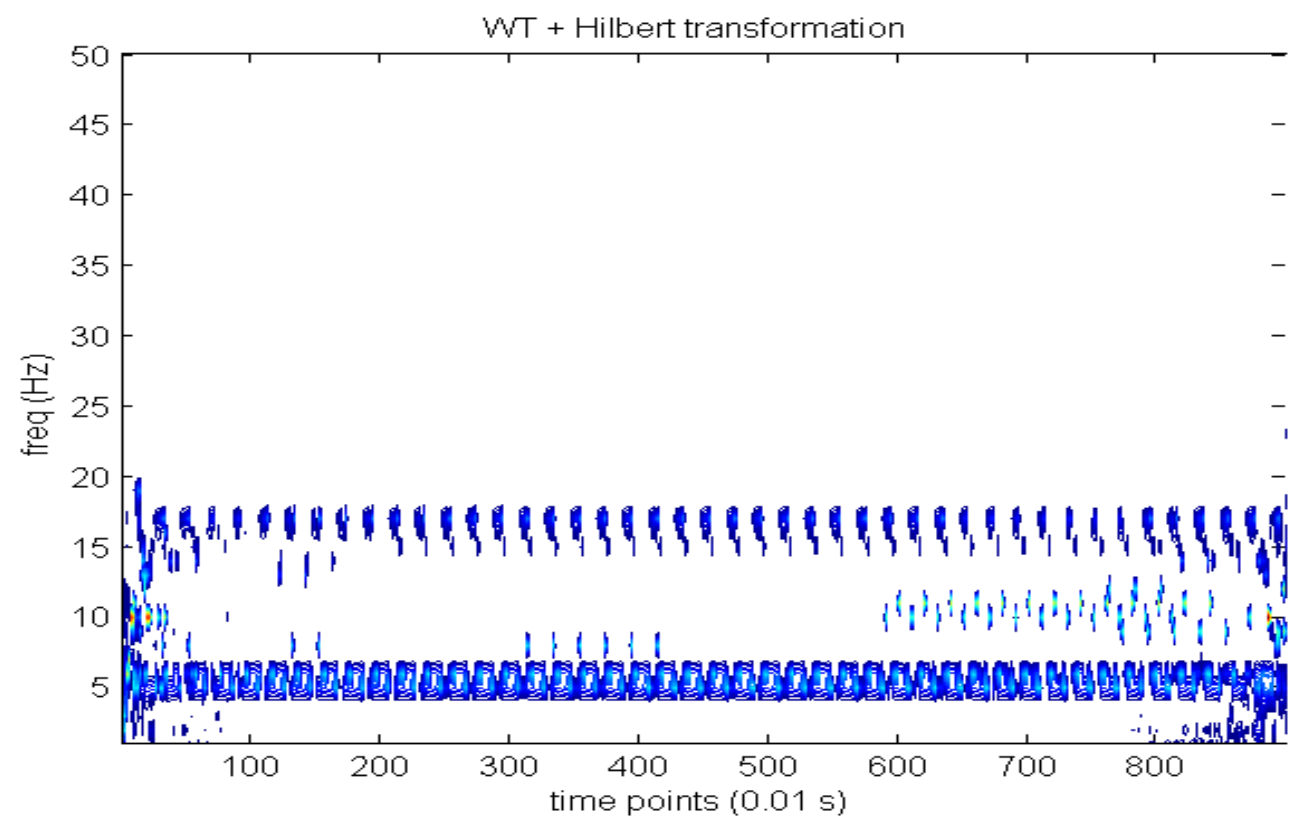

Figure 4.7d. WT + Hilbert transform for nonlinear signals. 


\section{MODE SEPARATION OF LAMB WAVES}

Mode separation for acoustics in tube-like structure is very important for feature extraction in structural monitoring. The raw signals and the separated signals are plotted in Figures 5.1 and 5.2 for comparison. In the raw signals, several modes of acoustic waves interweave together. The local peaks are irregularly distributed along the time axes. This makes the signals very difficult to be analyzed. However, the separated signals with single mode shown in Figure 5.2 have regular local peaks and clear patterns that are suitable for analysis using the moving window elaborated above.

In order to separate symmetric and anti-symmetric modes, a special sensor deployment is required, where two passive sensors are embedded into a tube, 180-deg apart at the same axial position. The independent pure mode waves are then calculated by:

$$
\begin{aligned}
& S_{a}=\left(s_{1}-s_{2}\right) / 2, \\
& S_{s}=\left(s_{1}+s_{2}\right) / 2, \\
& \text { where: } \\
& s_{i}: \text { raw singal from sensor } i, \\
& s_{a}: \text { antisymmetric singals, } \\
& s_{a}: \text { symmetric mode singals. }
\end{aligned}
$$




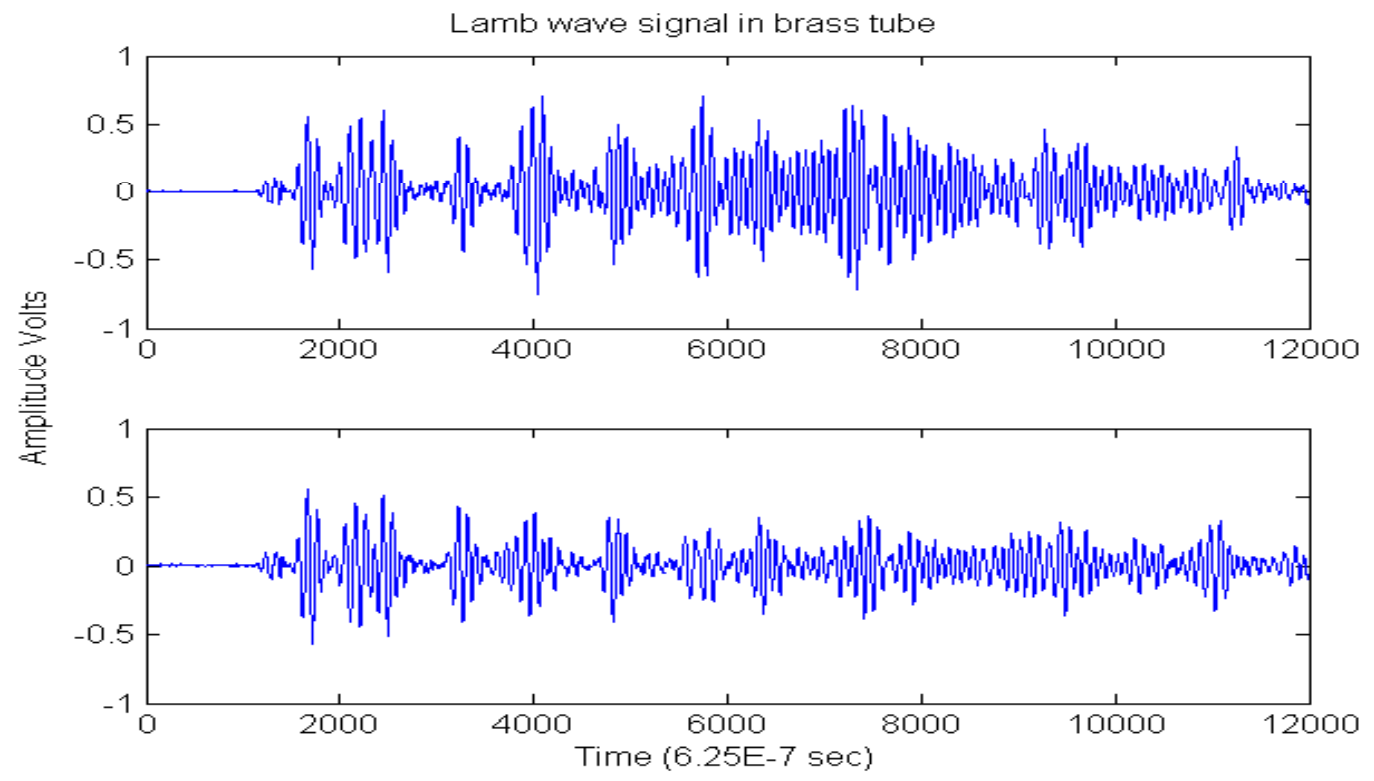

Figure 5.1. Raw signals from brass tube. 


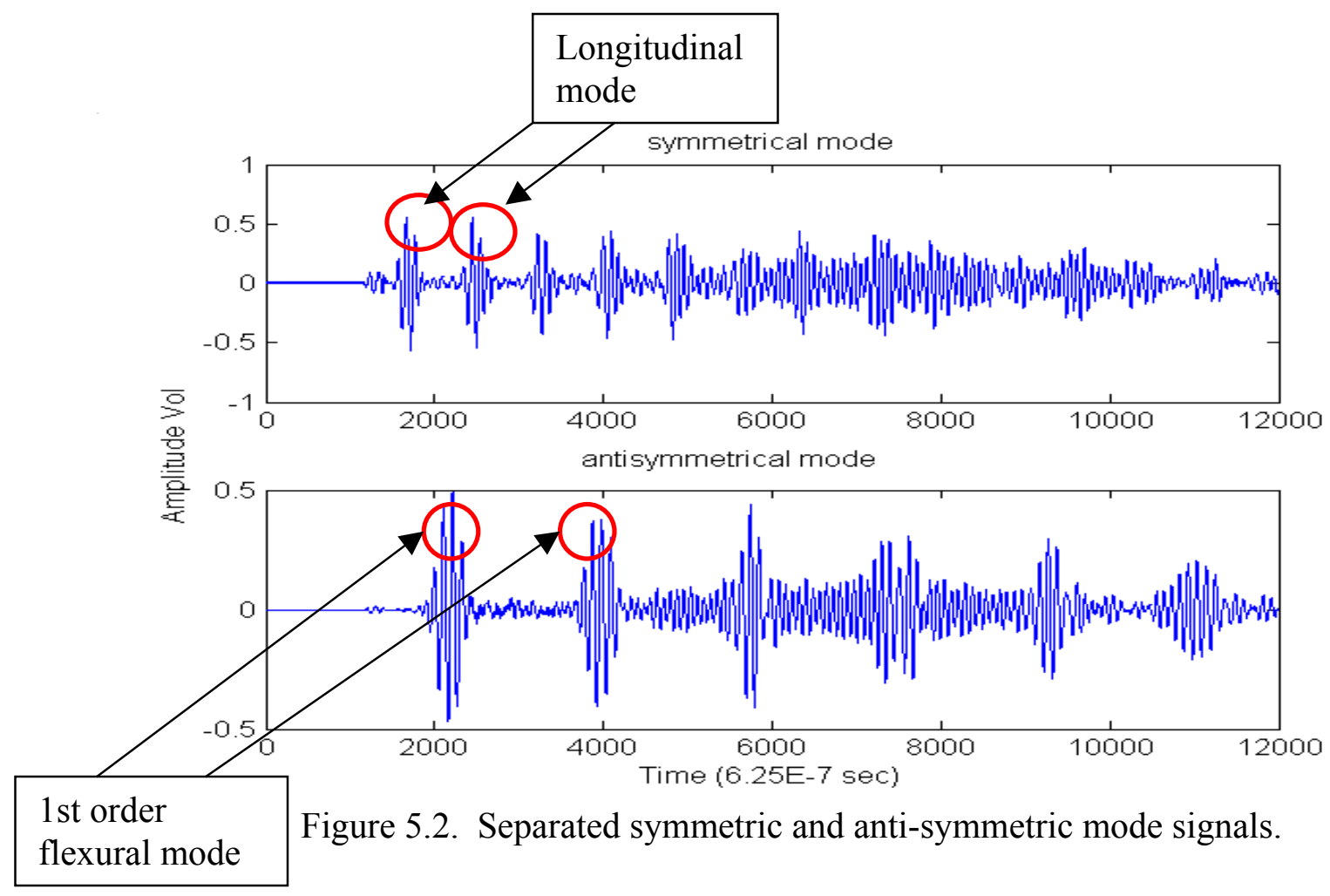

As we mentioned in Section 3, the Lamb wave propagation speed in a brass tube used in our research was verified using experimental data. With the separated mode illustrated in Figure 5.2, we can calculate the symmetrical mode speed by measuring the distance between two neighboring peaks and the length of the experimental tubes which is $0.9 \mathrm{~m}$. The average time interval between two neighboring peaks is $780 / 1600000=$ 4.8750e-4 second, so the symmetrical wave speed is $2 L / \Delta t=1.8 /\left(4.875 \times 10^{-4}\right)=3692.3 \mathrm{~m} / \mathrm{s}$. From Section 3, we know that the longitudinal mode speed (numerical solution) for the brass tube used in this research is $3,720 \mathrm{~m} / \mathrm{s}$, therefore we can decide that the symmetric mode appeared on the upper plot in Figure 5.2 is a longitudinal mode.

In a similar fashion, the anti-symmetric mode speed may be calculated. The average time interval in the lower plot in Figure 5.2 is $1874 / 1600000=0.0012$ second. Hence the anti-symmetrical wave speed is $2 L / \Delta t=1.8 / 0.0012=1536.8 \mathrm{~m} / \mathrm{s}$. The theoretical speed for the first-order flexural mode calculated in Section 3 is about $1500 \mathrm{~m} / \mathrm{s}$, and it is the only flexural mode that exists at $13 \mathrm{kHz}$. Therefore, the antisymmetrical wave in the lower plot of Figure 5.2 is the first-order flexural mode 
propagating along a brass tube with inner radius $5.1 \mathrm{~mm}$ and outer radius $6.35 \mathrm{~mm}$. The small difference between experimental and the theoretical results are due to several reasons. The first reason is that the length of tube is an approximate value. A small measurement error such as $9 \mathrm{~mm}$ will cause about $30 \mathrm{~m} / \mathrm{s}$ difference in longitudinal speed. The second reason comes from the measurement of the inner and outer radius of the experimental tubes. These two dimensions are two sensitive parameters in the Lamb wave numerical estimation. The third source of error is from the numerical calculation itself. This error may be reduced by the enhancement of eigen-value algorithm, but cannot be completely eliminated.

Therefore, the virtues of separation of the Lamb wave in tubular structures exist not only in simplifying the Lamb wave structures, but also verifying the experimental results through theoretical analysis. The advantages of this technique will be further illustrated in the tubular structure health evaluation described in Section 7. 


\section{STRUCTURAL DIAGNOSTICS OF ALUMINUM PLATES}

Experimental analysis of aluminum plates in air was performed with different input frequencies. The length of the aluminum beams used in this study is two feet. As the optimal selection, $27 \mathrm{kHz}, 28 \mathrm{kHz}, 29 \mathrm{kHz}$, and $30 \mathrm{kHz}$ sine pulses, modulated by Hanning windows are used to stimulate the piezo-transducers attached on to the surface of the metal beam to produce Lamb waves. A typical output from three passive channels is plotted in Figure 6.1. The $3^{\text {rd }}$ sensor and the $4^{\text {th }}$ sensor are placed back to back on two opposite faces of the aluminum plate at the same position. This allows us to separate the symmetric and anti-symmetric modes of propagation as shown in Figure 6.2.
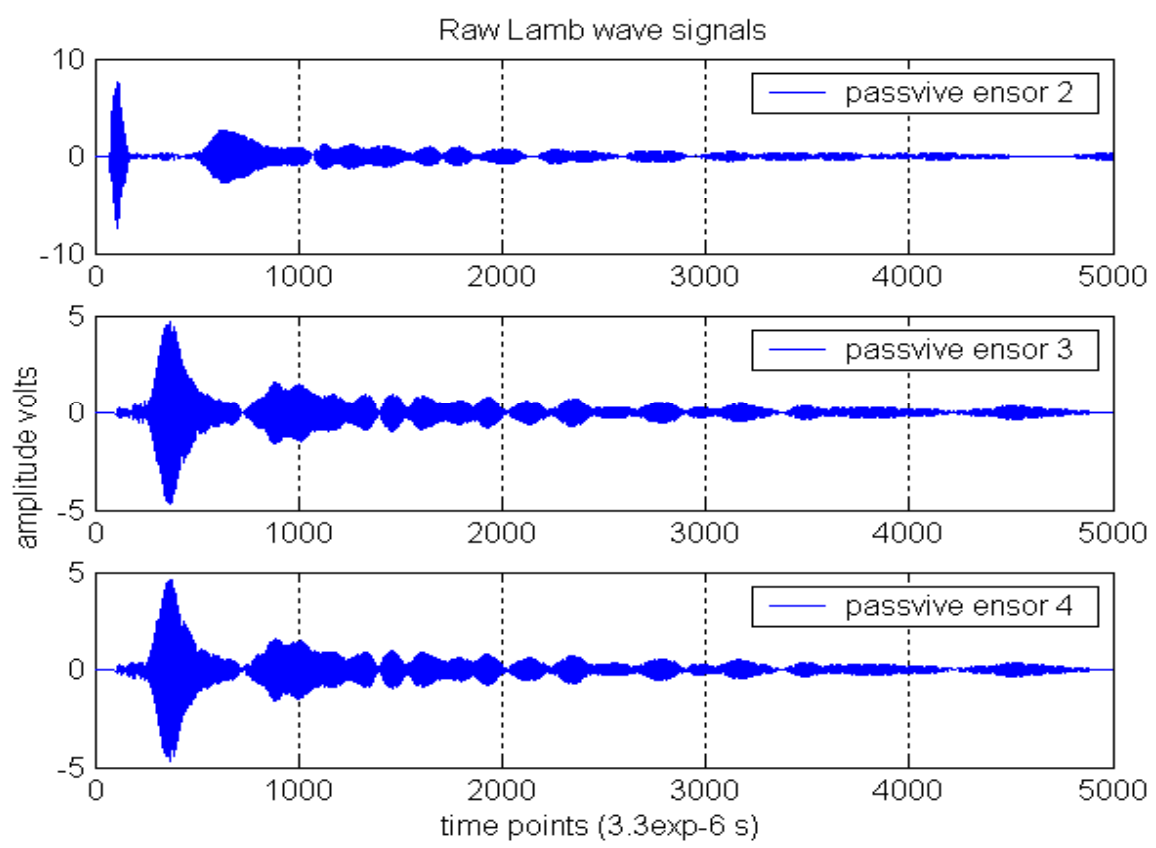

Figure 6.1. Lamb wave signals from an aluminum plate. 


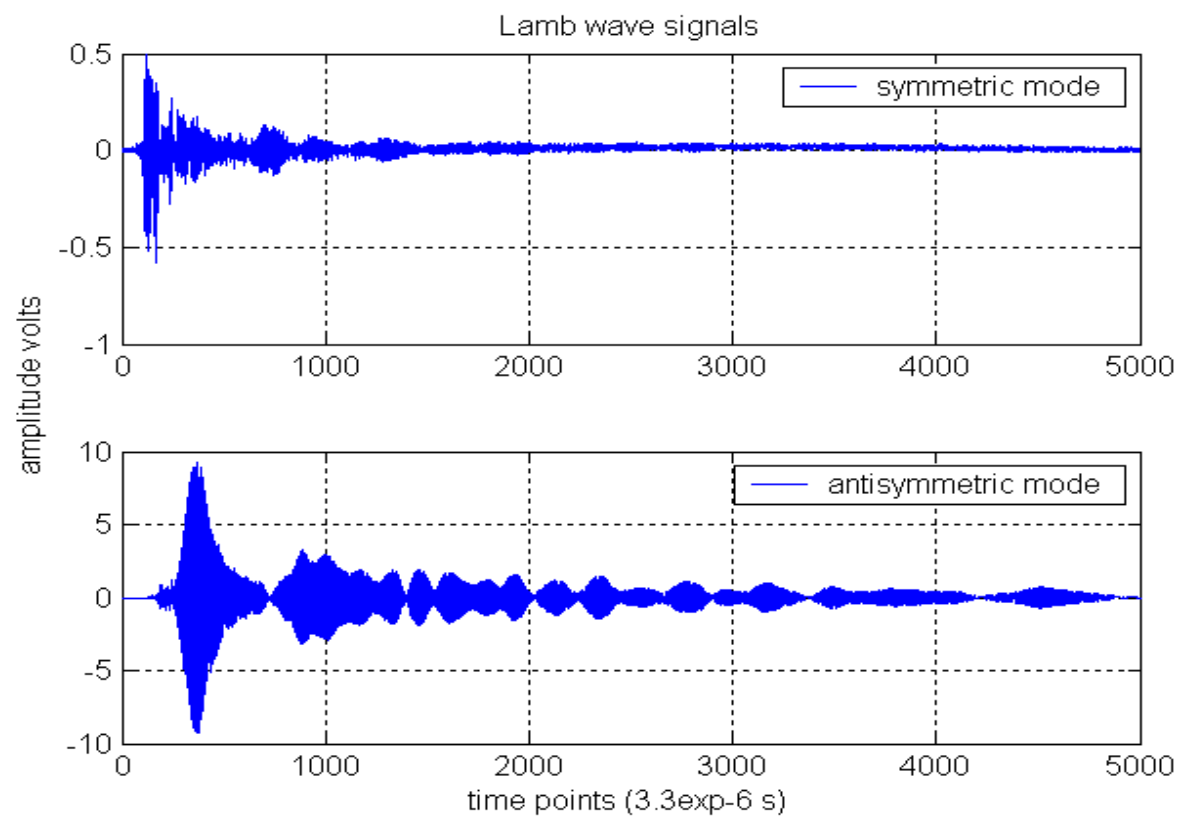

Figure 6.2. Symmetric and anti-symmetric modes of waves in a plate.

It is noticed by comparing the separated signals that the symmetric mode wave is very weak compared with the anti-symmetric mode, and the symmetric mode propagates faster than the anti-symmetric mode. Since in this study, the anti-symmetric mode dominates the wave signal in an aluminum plate, the following analysis focuses on antisymmetric mode or raw signals without reference to symmetric signals. However, it does not mean that the symmetric wave is not important. With appropriate excitation, we are able to generate pure symmetric mode Lamb waves in plate-like structures, and the analysis of mode conversion between symmetric and anti-symmetric modes are of importance in structural flaw detection.

\subsection{Flaw Detection and Localization Using HHT}

Structural flaw detection for an aluminum plate was discussed in the 2003 Annual Report [118], using the energy ratio method and DWT analysis. There are several other studies focused on flaw detection for plate-like structure. Although the focus of this research is to monitor flaws in tubing (in the air and in water), the study on aluminum 
plates has been performed because of its simple geometry and fewer number of wave modes.

As mentioned above, the Hilbert-Huang transform emphasizes instantaneous frequencies, and can generate clearer time-frequency plots in many circumstances. In addition, due to the intrinsic decomposition, HHT often extracts more meaningful features than other algorithms such as the WT. The sifting process used in the HHT decomposes the signal into important components. The sifting process uses local extrema and cubic spline interpolation to sift out local high frequency waves, therefore HHT is really adaptive to the signals itself, that is, it has good nonlinear properties. In summary HHT provides us a powerful non-stationary, nonlinear DSP method to deal with both intra-modulated and inter-modulated signals. As an example, Figure 6.3 illustrates the sifting processing of Lamb wave signals from an aluminum plate. The original signal is plotted in Figure 6.4. It is very important to notice the adaptive characteristics of HHT, displayed by each intrinsic mode function (IMF). We see that an IMF need not be of narrow band in the whole time domain, but must be of narrow band at each time instant. This property is extremely useful in filtering out nonlinear and nonstationary background noise in steam generators as illustrated for tubing structural diagnostics in the Section 7. 


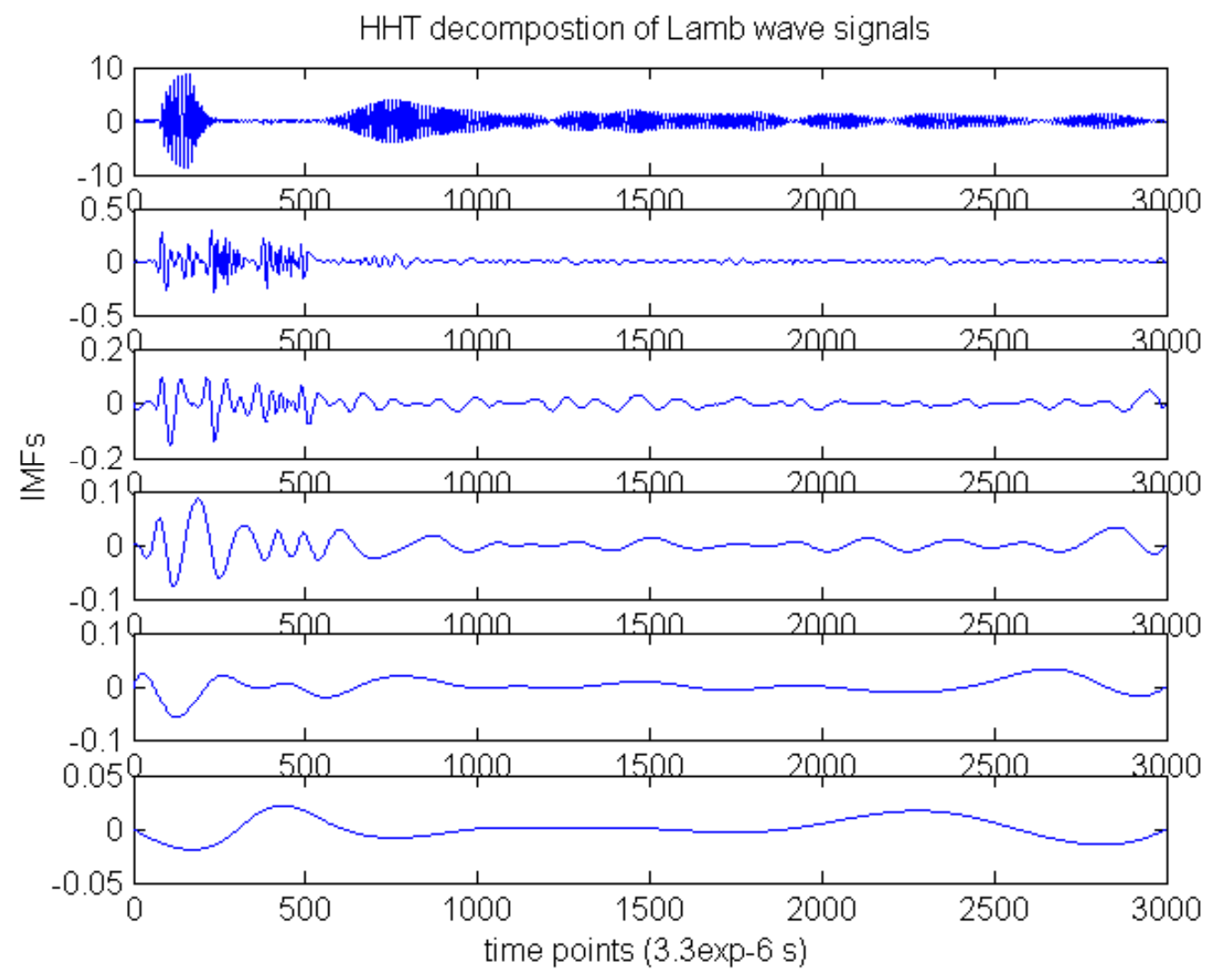

Figure 6.3. HHT decomposition of Lamb waves in a plate. 


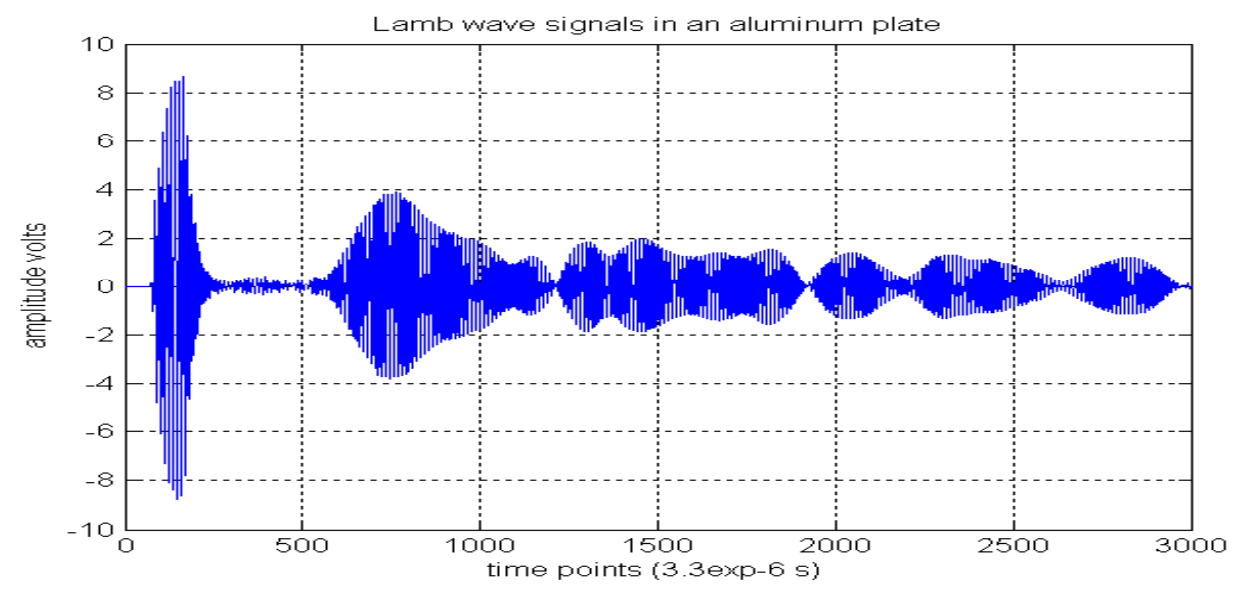

Figure 6.4. Lamb wave signal in an aluminum plate.

As illustrated in Figures 6.5 and 6.6, the HHT generates clear time-frequency plots of Lamb waves for the aluminum plate. It was used to indicate the potential structural problems in this research and is found to be more sensitive in some cases than other methods, especially for the small flaw such as a partial hole on the surface of the aluminum plate. Another method described next will not be able to detect this type of small flaw, but can detect other kinds of flaws such as clips, deep v-notch, etc. The flaw position can be estimated using the distance between the reflected signals indicated in Figure 6.6 and the first group of peaks or the second group of peaks, which correspond to the reflection from the boundaries of the beam. The estimated position of the defect in Figure 6.6 should then be at one-third of the plate length from the left boundary, which is very near the actual flaw position.

An important information revealed by the HHT plots is the dispersion phenomena of Lamb waves in plate like structures. In our experiments, we tried to create active signals with pure frequency using modulated sine wave (Hanning window); however, the finite length of modulated window will definitely cause the frequency spread due to the effect of window's boundary. Therefore we find that the Lamb wave signals are concentrated around the selected center frequency of input signals, which is $27 \mathrm{kHz}$ for Figures 6.5 and 6.6. However, there are other frequency components around $27 \mathrm{kHz}$, and their transportation speeds are inversely proportional to their frequencies. This proves that the Lamb waves here are in an anti-symmetric mode, because only the anti- 
symmetric $0^{\text {th }}$ order mode has this relationship between frequency and wave speed in low frequency bands, whereas the symmetric $0^{\text {th }}$ order mode has constant speed in low frequency bands.

Figures 6.7 to 6.12 illustrate more experimental Lamb waves and their HHT under different structural conditions. We can see that the HHT generates clear time-frequency plots for aluminum plates, and indicates the structural anomaly without complexity. The approximate flaw position may be estimated more accurately in the HHT plot than in the raw signal plot. The HHT is therefore sensitive to small signal changes in both the time and the frequency domains, while the WT analysis does not perform well based on the test that we performed. The reason is WT has more energy leakage than HHT, so the WT may not be sensitive to the small reflection from the structural flaw.

One problem associated with the structural diagnostics described above using HHT is the estimation of the flaw size. We can roughly state that the flaw size is proportional to the energy of the reflected wave; however, the reflection is also affected by the position of the flaw. The method used to estimate the flaw size in a systematic way for plate and tubular structure deserves to be further studied.

Another nonlinear signal processing technique for structural flaw detection in plates is elaborated in the next section, which can estimate the flaw position more quantitatively. 


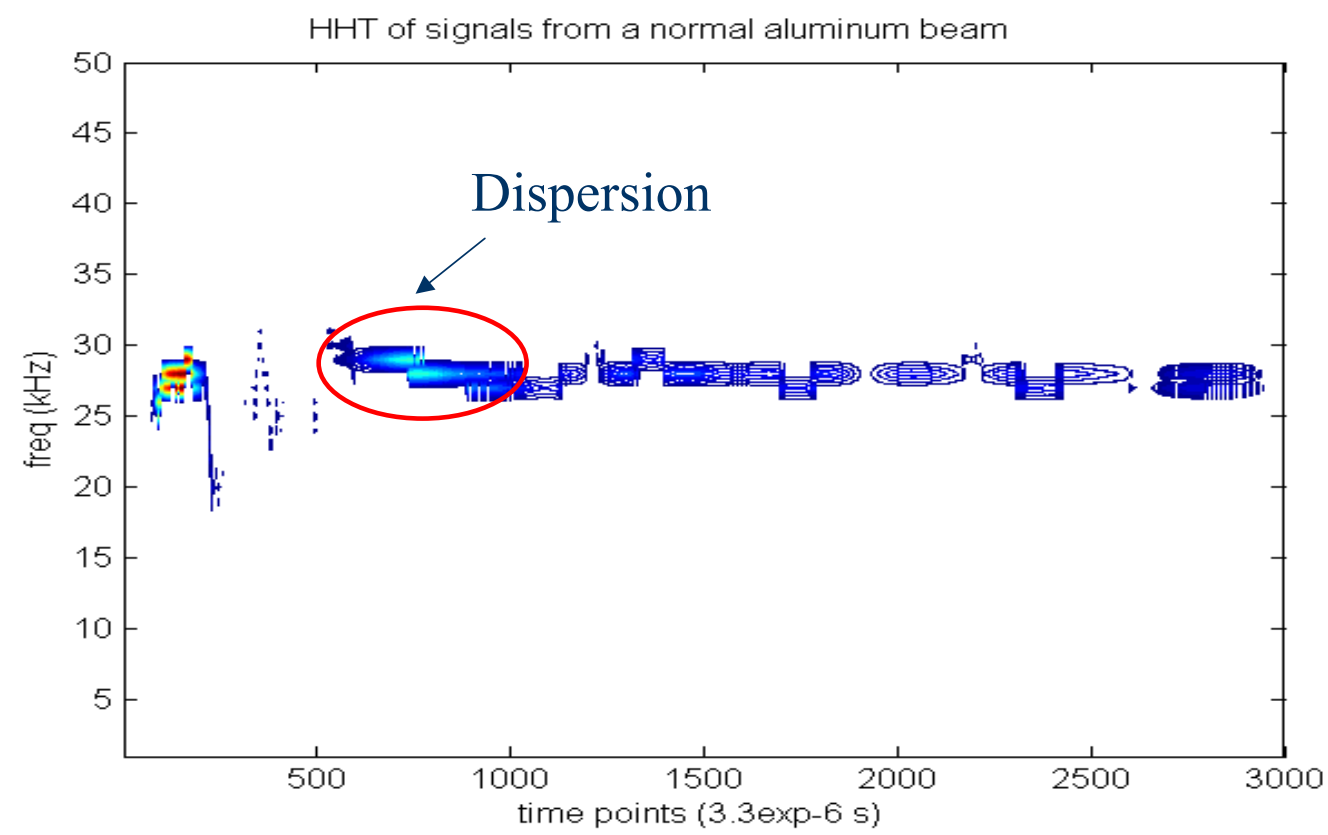

Figure 6.5. Time-frequency representation of HHT of Lamb wave signal in a normal aluminum plate.

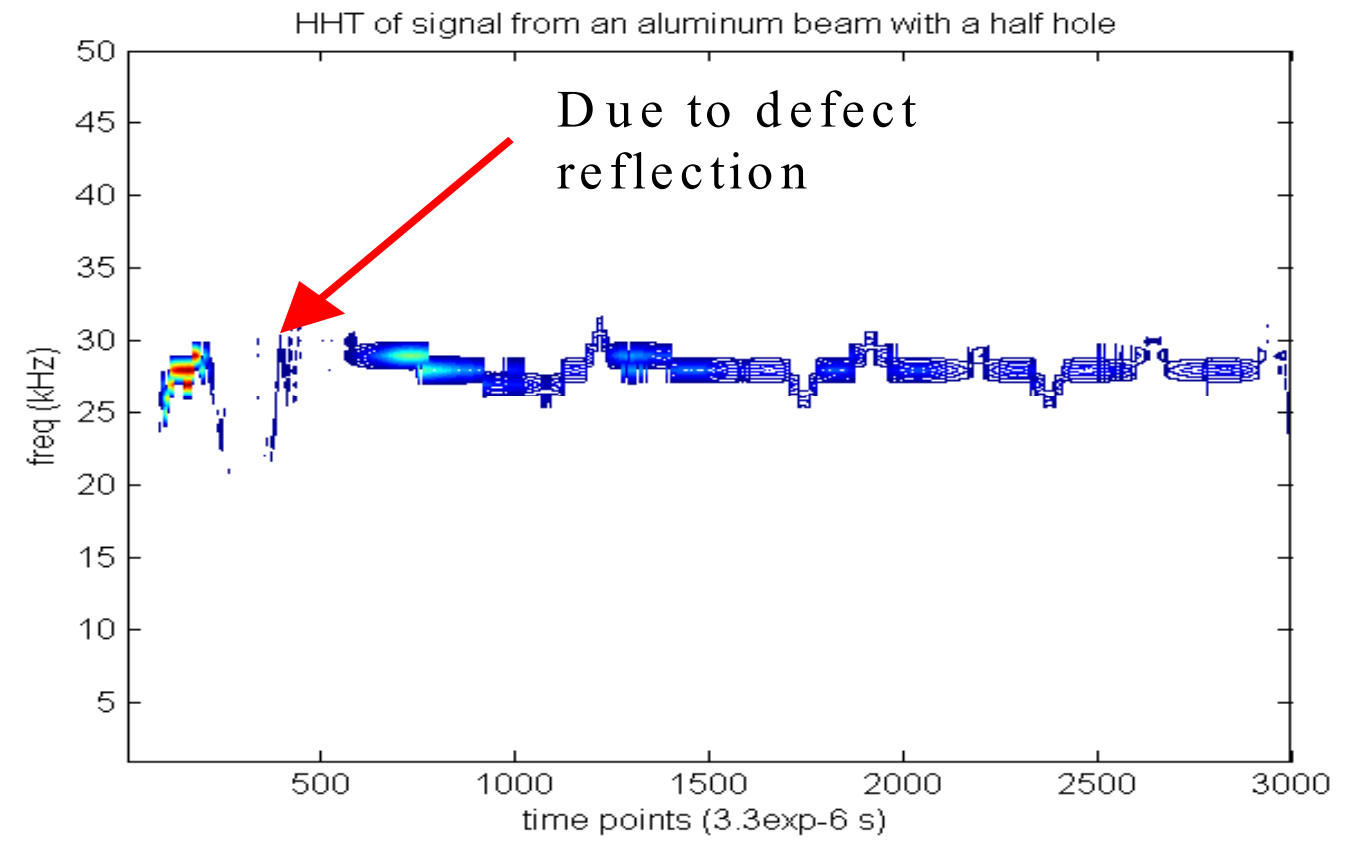

Figure 6.6. Time-frequency representation of HHT of Lamb wave signal in an aluminum plate with a partial hole. 

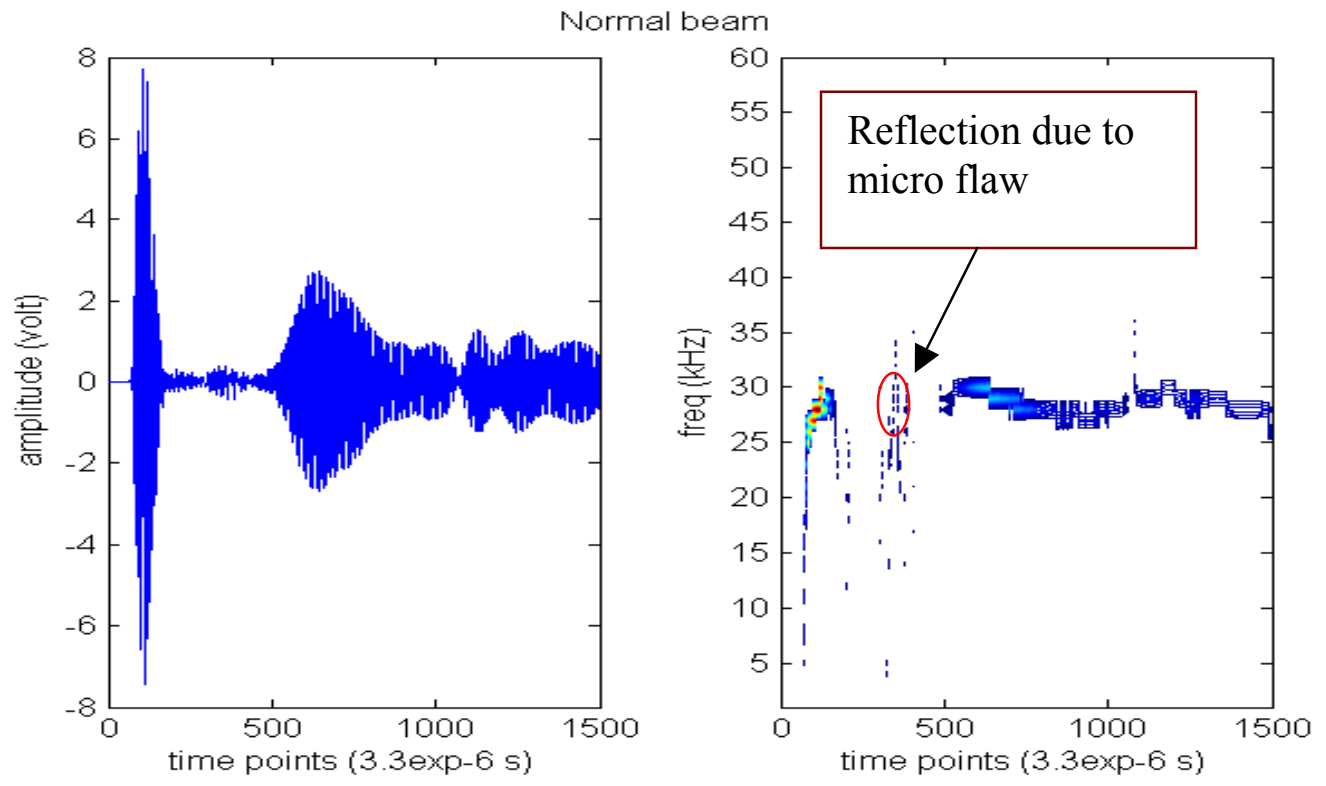

Figure 6.7. Normal Lamb wave signal and its HHT.

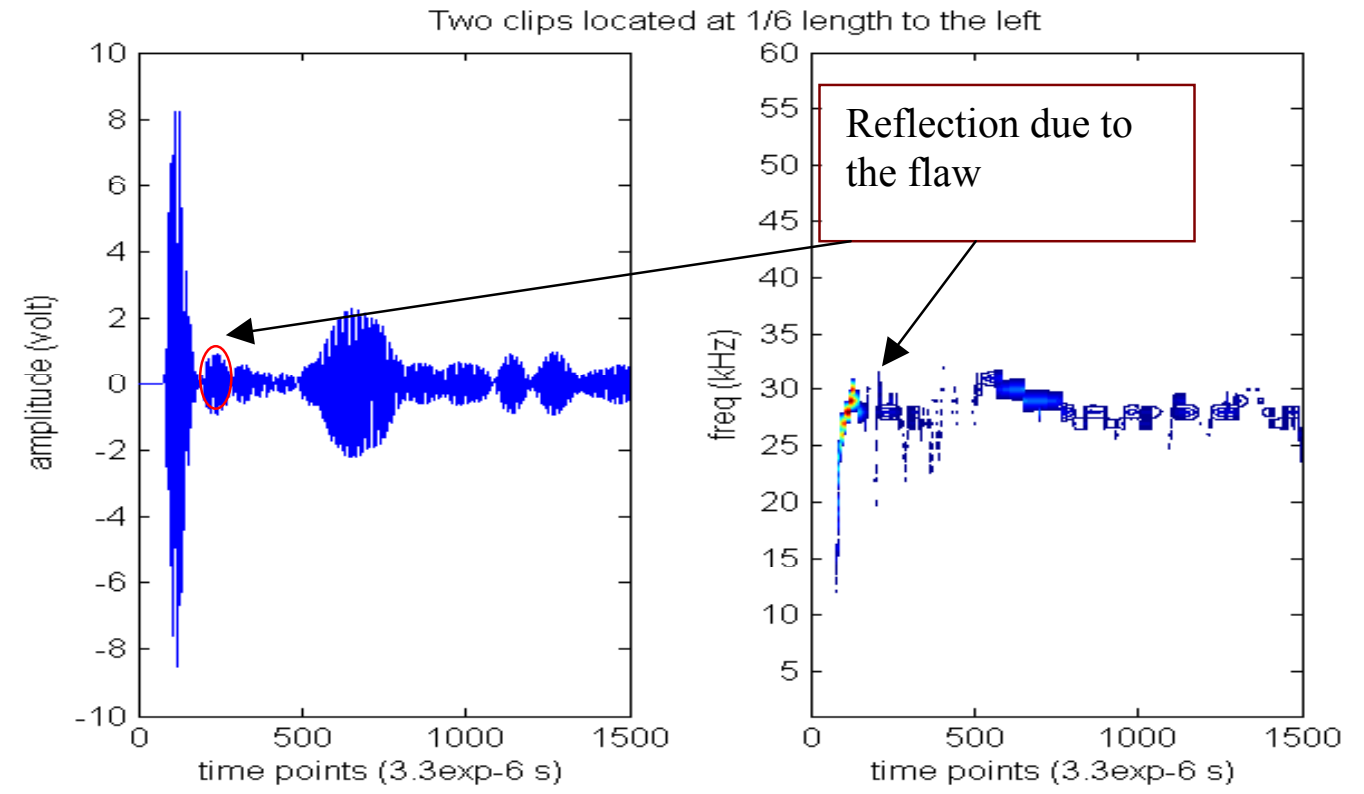

Figure 6.8. Lamb wave signal from an aluminum beam with two clips located near the left end and its HHT. 


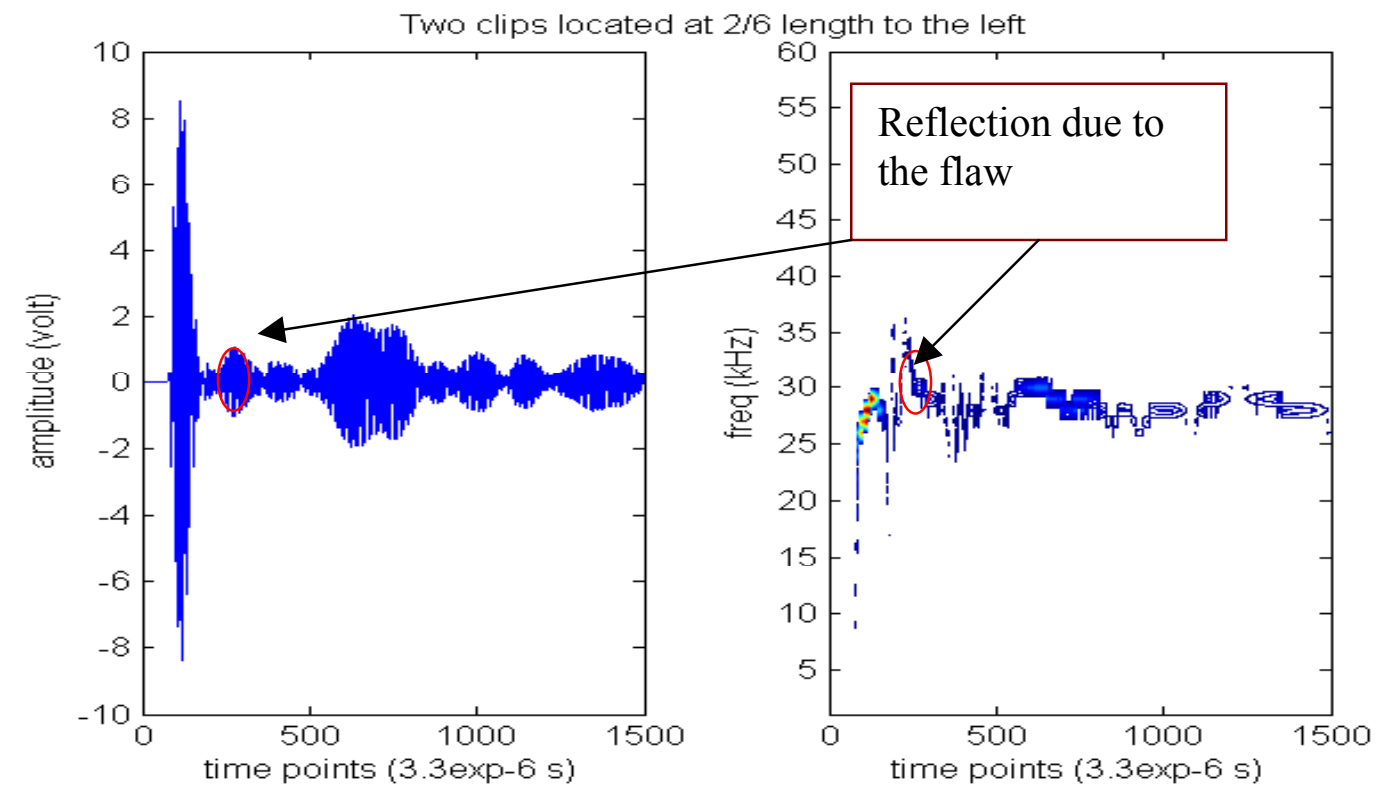

Figure 6.9. Lamb wave signal from an aluminum beam with two clips located near the left end and its HHT.

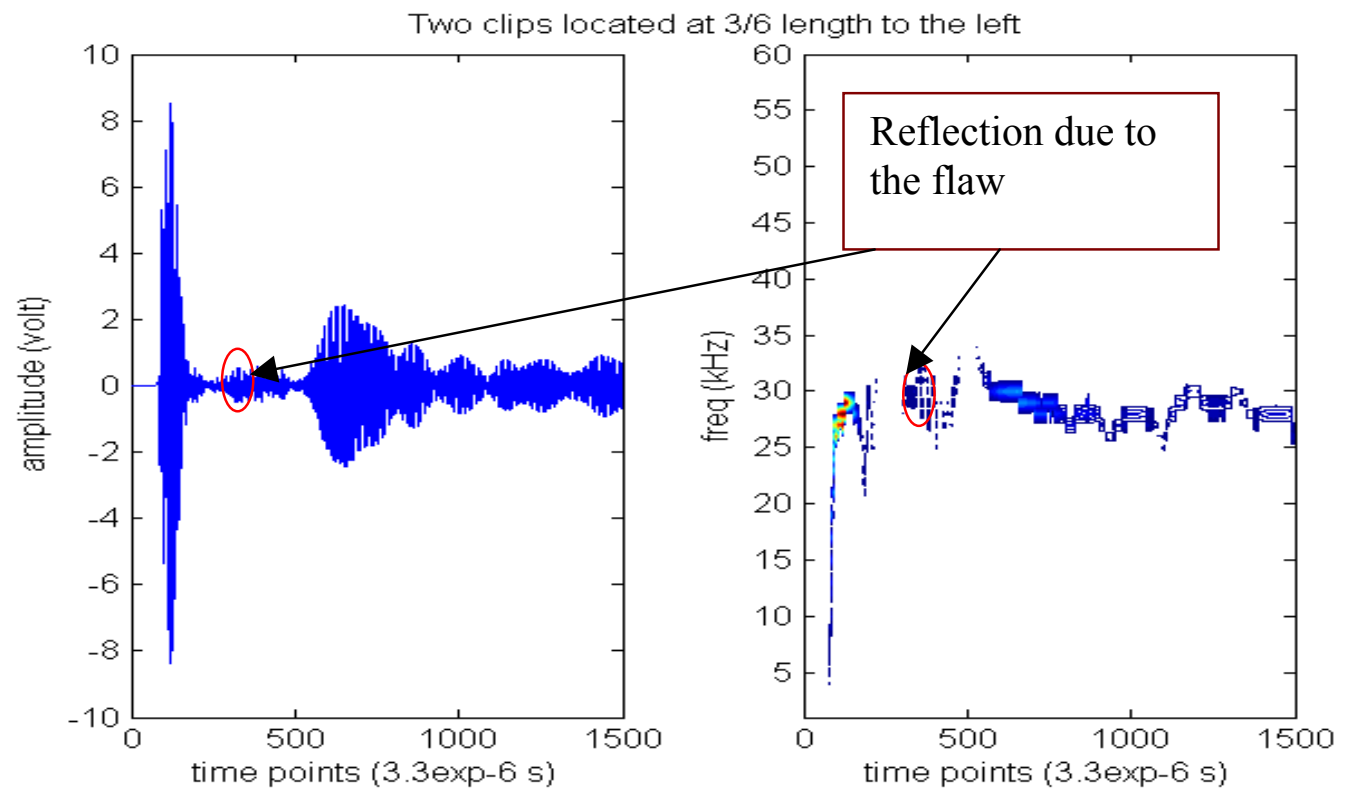

Figure 6.10. Lamb wave signal from an aluminum beam with two clips located in the middle and its HHT. 


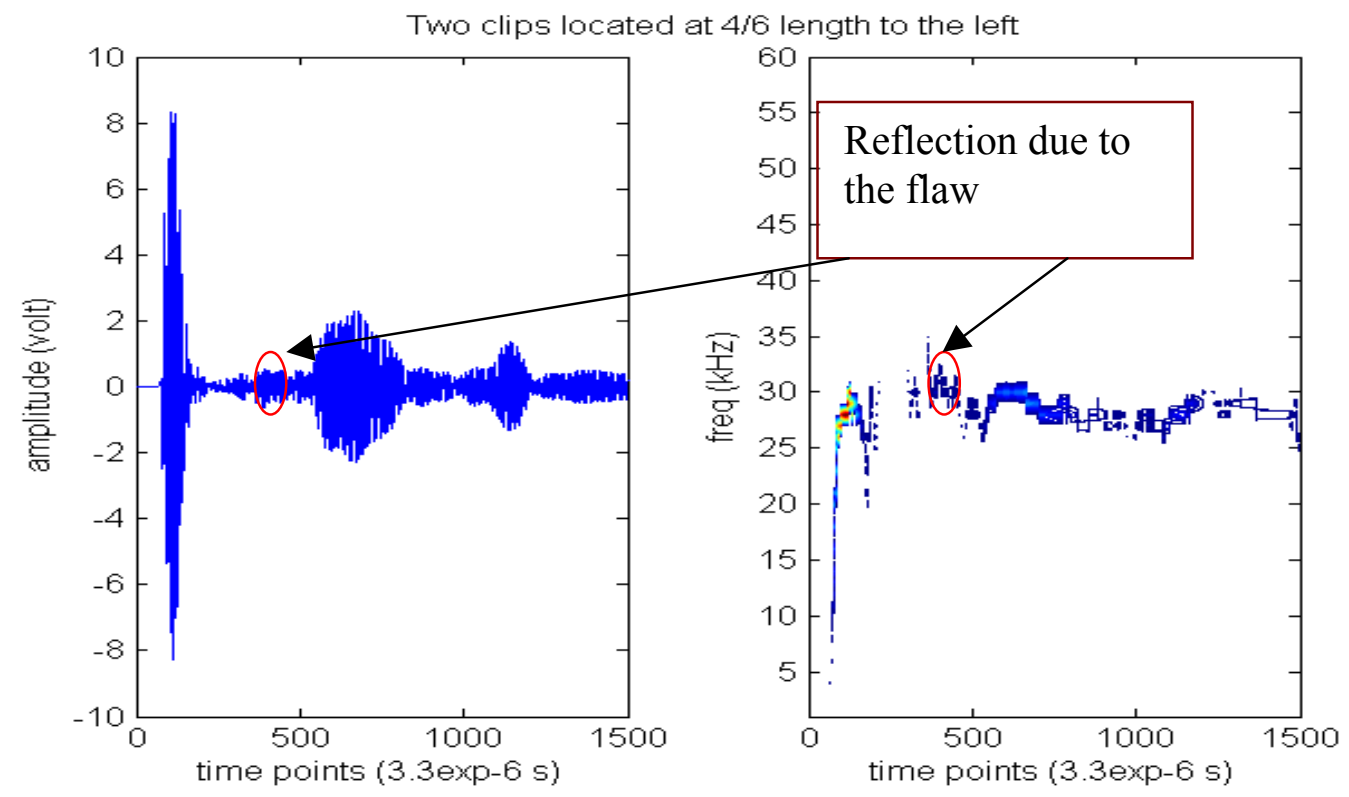

Figure 6.11. Lamb wave signal from an aluminum beam with two clips located near the right end and its HHT.

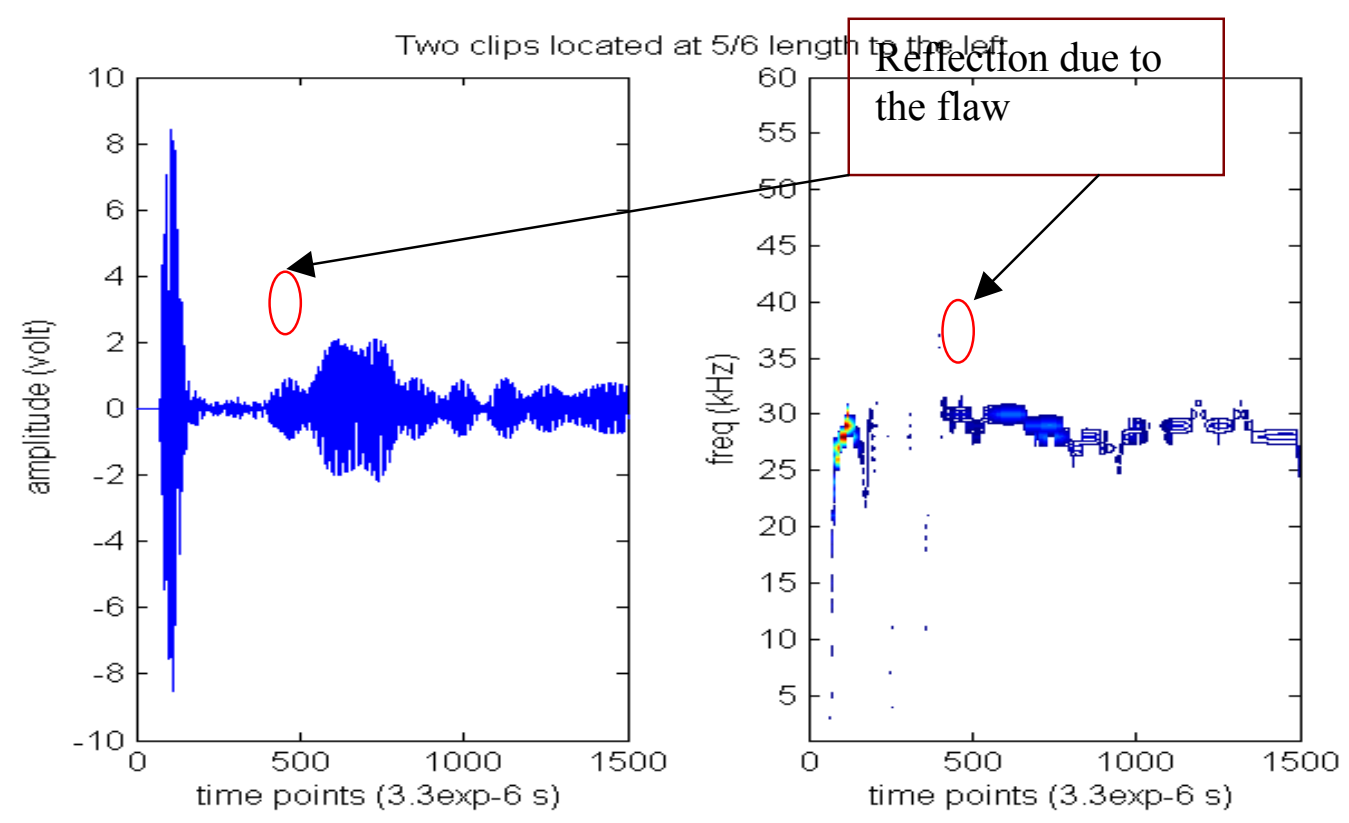

Figure 6.12. Lamb wave signal from an aluminum beam with two clips located near the right end and its HHT. 


\subsection{Flaw Detection and Localization Using Extrema Extraction}

As discussed in the HHT analysis, the HHT provides us with an effective way to understand the Lamb waves in a plate like structure. It is very sensitive to the changes caused by small flaws especially surface flaws. HHT can even be used for testing the homogeneity of materials. For instance, in Figure 6.5, the aluminum plate is supposed to be flawless, but we can still find some small spots between the first group of peaks and the second group. These spots are not caused by macro-structural flaws, but by the micro-structural change, such as a single broken fiber. Hence the Lamb wave is sensitive enough to feel the stress distribution change, and HHT is able to extract the micro-changes from the signals. In addition, the resolution of the contour plot of T-F distribution from HHT can be easily adjusted according to the requirement of detection.

However, there are also other analytical methods that can be used to extract representative features of Lamb waves; for example, the method of extrema extraction used below. For some types of flaws, such as clip and v-notches, this method is very effective. Compared with the HHT analysis, this is a simple and effective method to save calculation time without degrading the results of diagnostics. The method is not very sensitive to small surface flaws, but still a good check for the HHT analysis.

The objective of extrema extraction is to extract the upper and lower envelopes from Lamb wave signals. Since any structural flaw will change the signals collected by the passive sensors, the shapes of the envelopes would be affected. The comparison between normal wave envelope and the abnormal wave envelope should reveal the structural change in the materials.

In order to extract the envelope of a time series, first the local maxima and minima are picked out separately. The points between the maximum points are estimated using a cubic-spline interpolation to generate an upper envelope. In the same way, the interpolation of minimum points gives the lower envelope. An example of the extraction process is shown in Figure 6.13.

Passive wave signals under different system conditions are compared in Figure 6.14, where the blue line corresponds to the normal condition and the other three correspond to the system response under the same flaw ( 2 clips on) but different positions 
on the plate. The first one (green line) is located at $1 / 5$ of the plate length from the active sensor; the second is located at $2 / 5$ of the length from the active sensor; the third one is located in the middle of the test plate. The plot shows the defect on plate causes the amplitude to decrease more quickly. The first peak is the wave signals collected by the passive sensor before collisions. Therefore the amplitudes are almost the same.

The signals between the first peak and the second peak are plotted in Figure 6.15. The location of flaw can be estimated approximately by the location of the peaks. As demonstrated in Figure 6.15, the peak of each curve is due to the reflection of structural flaw and thus the time of flight directly reflects the distance between the receiving sensor and the defect.

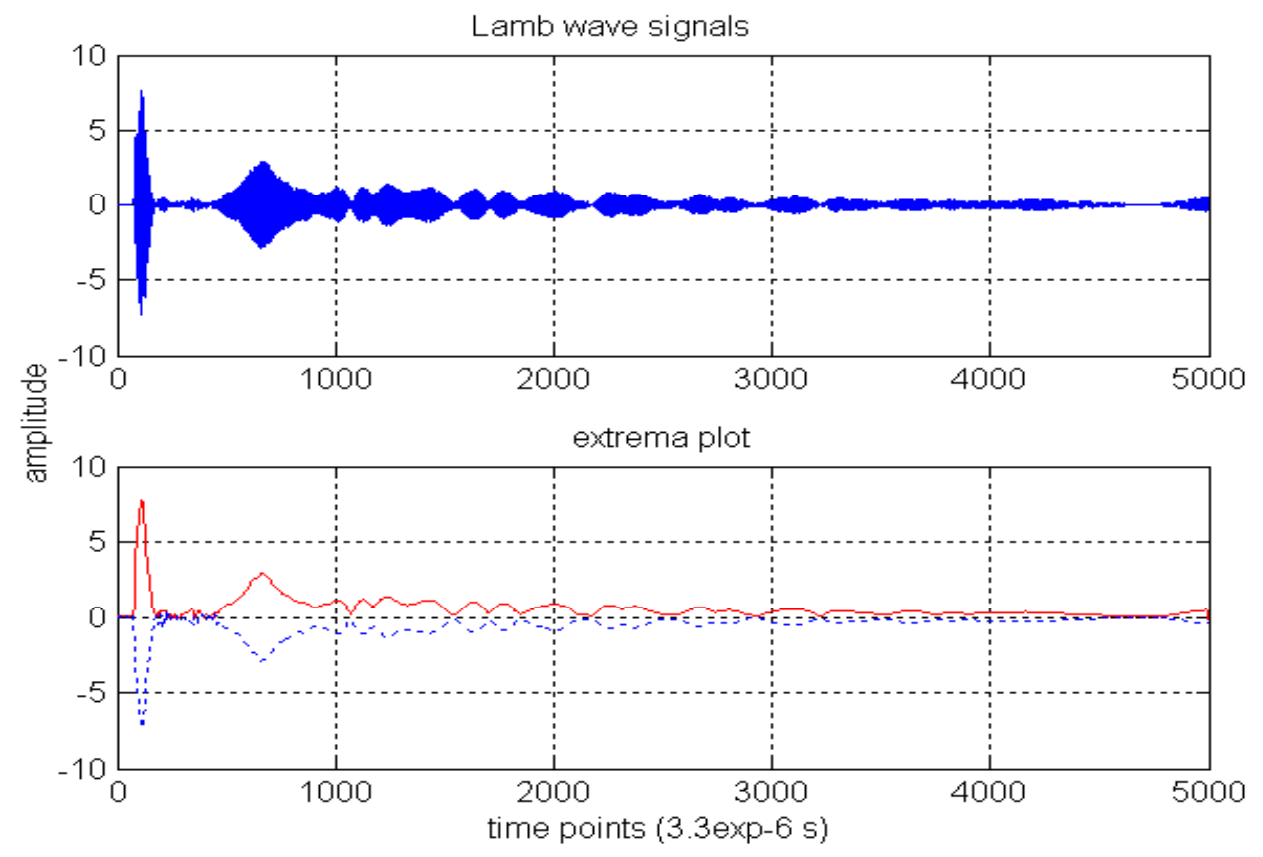

Figure 6.13. Lamb waves and envelope extraction. 


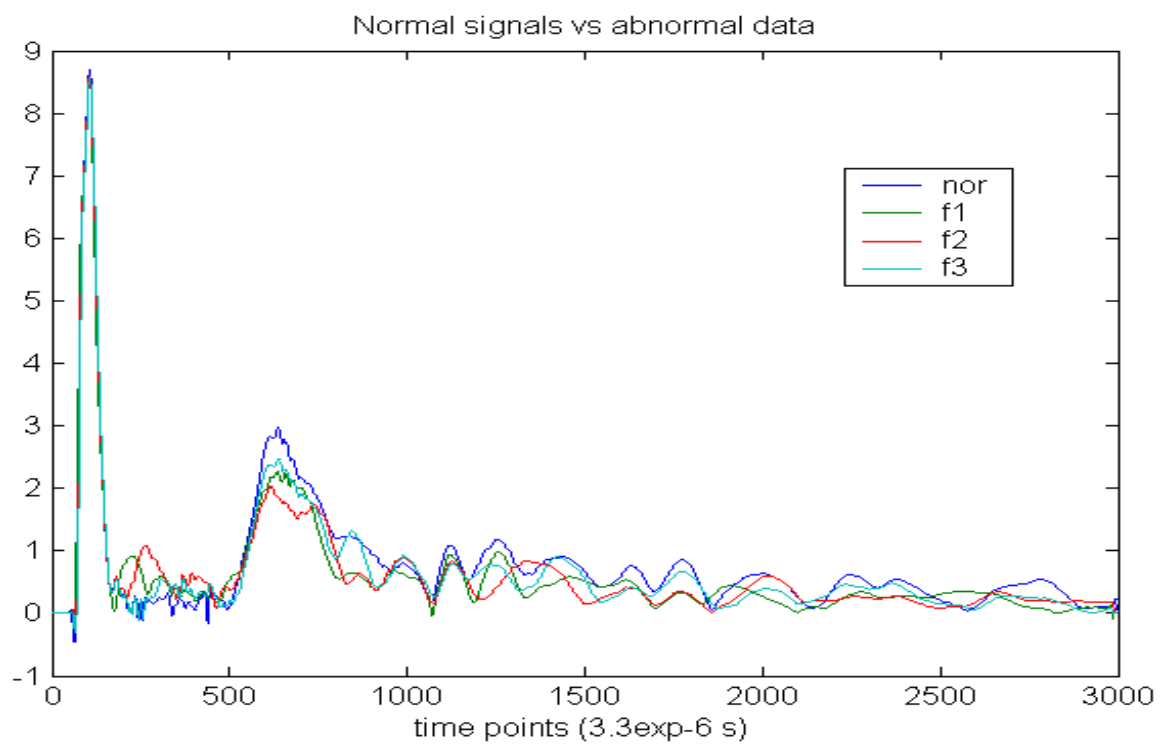

Figure 6.14. Passive Lamb wave signals for aluminum beam under different conditions.

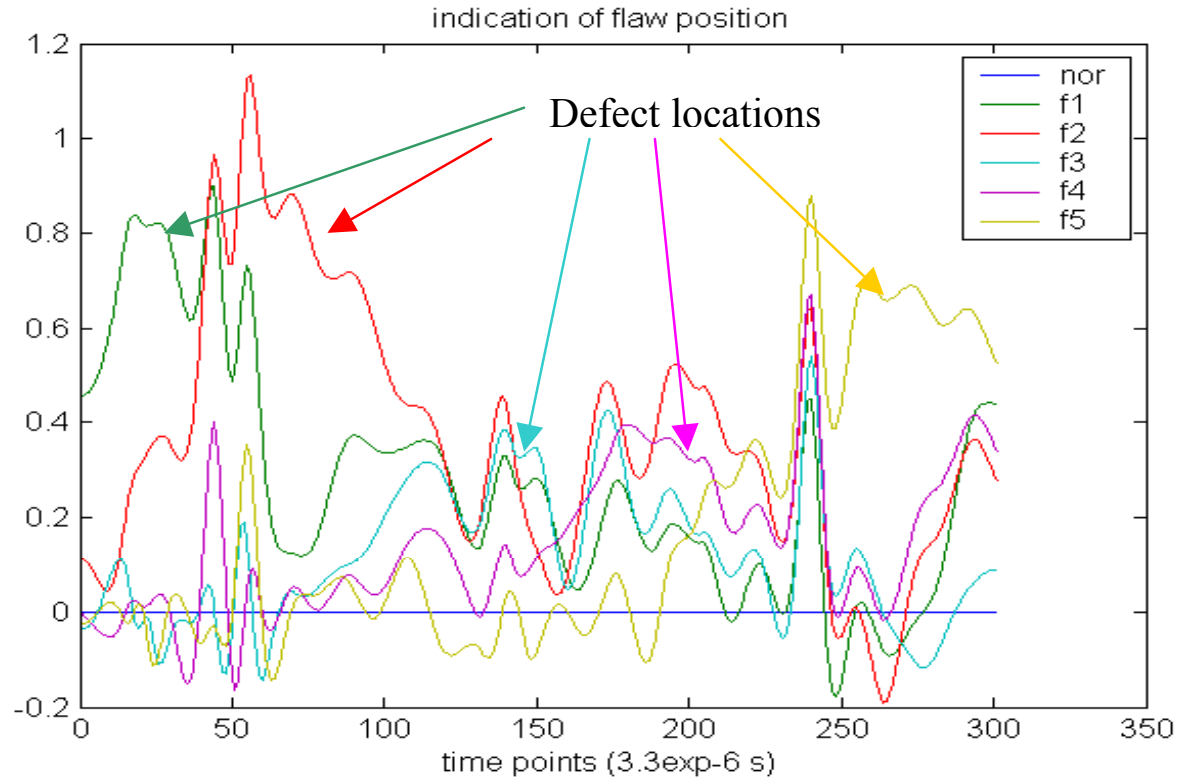

Figure 6.15. The signals between the first and the second peaks for different flaw types. 


\subsection{Selection of the Resonant Frequency for Aluminum Plate}

The selection of active frequency is very important for the implementation of guided acoustics. We want to use the frequency band that has low decay ratio, thus high signal-noise ratio in structural monitoring to decrease false rate and to realize remote monitoring. The resonant frequency is usually an optimal selection for the excitation signal. The resonant frequency of a tested specimen is decided by several parameters such as the length, shape, and materials. Two methods can be used to decide the resonant frequency of a tested structure. In the first method, we generate a gradually increasing chirp signal to scan a wide band of the input frequency. The resonance peaks can be decided by observing the output time signals plotted in Figure 6.16. Another method is to use HHT of a pure pulse signal as demonstrated in Figures 6.17 and 6.18. Since the pulse signal theoretically includes all the frequency information (limited by the sampling frequency), it is reasonable to find the resonant frequency from a proper time frequency plot. As shown in Figure 6.17, the HHT generates a clear time-frequency plot where it is easy to find not only the resonant frequency around $27 \mathrm{kHz}$ but also the dispersion curve of the anti-symmetrical Lamb wave signals. These two methods give out same results, but it is obvious that HHT is more concise.

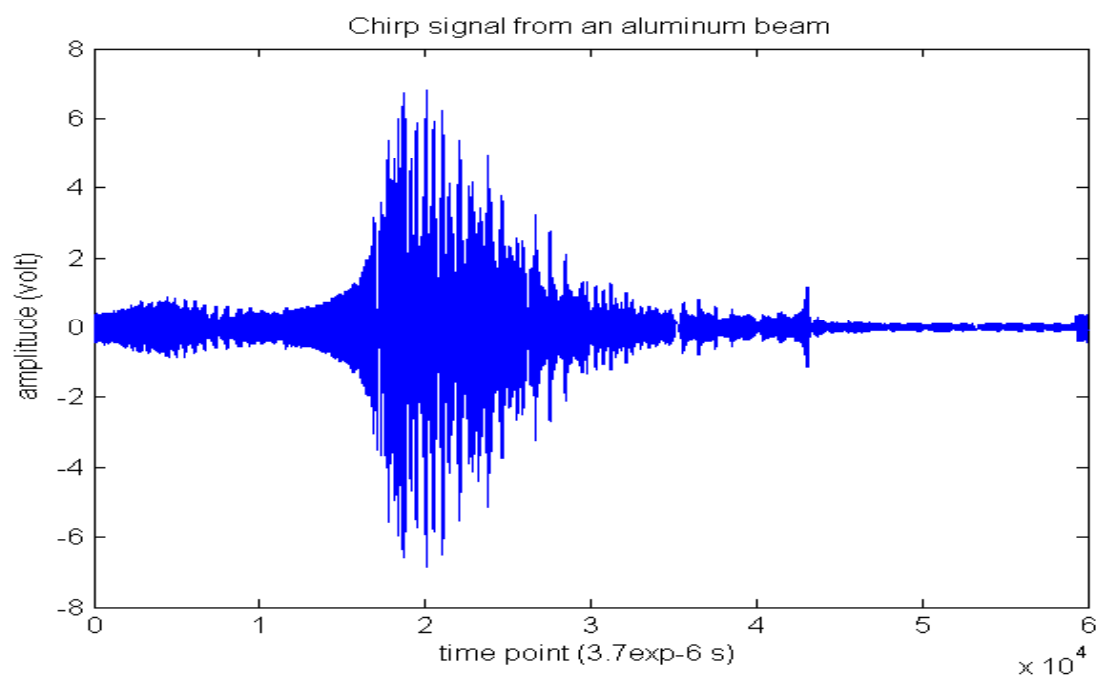

Figure 6.16. Chirp signal scanning a wide band from 100 to $90 \mathrm{k} \mathrm{Hz}$. 


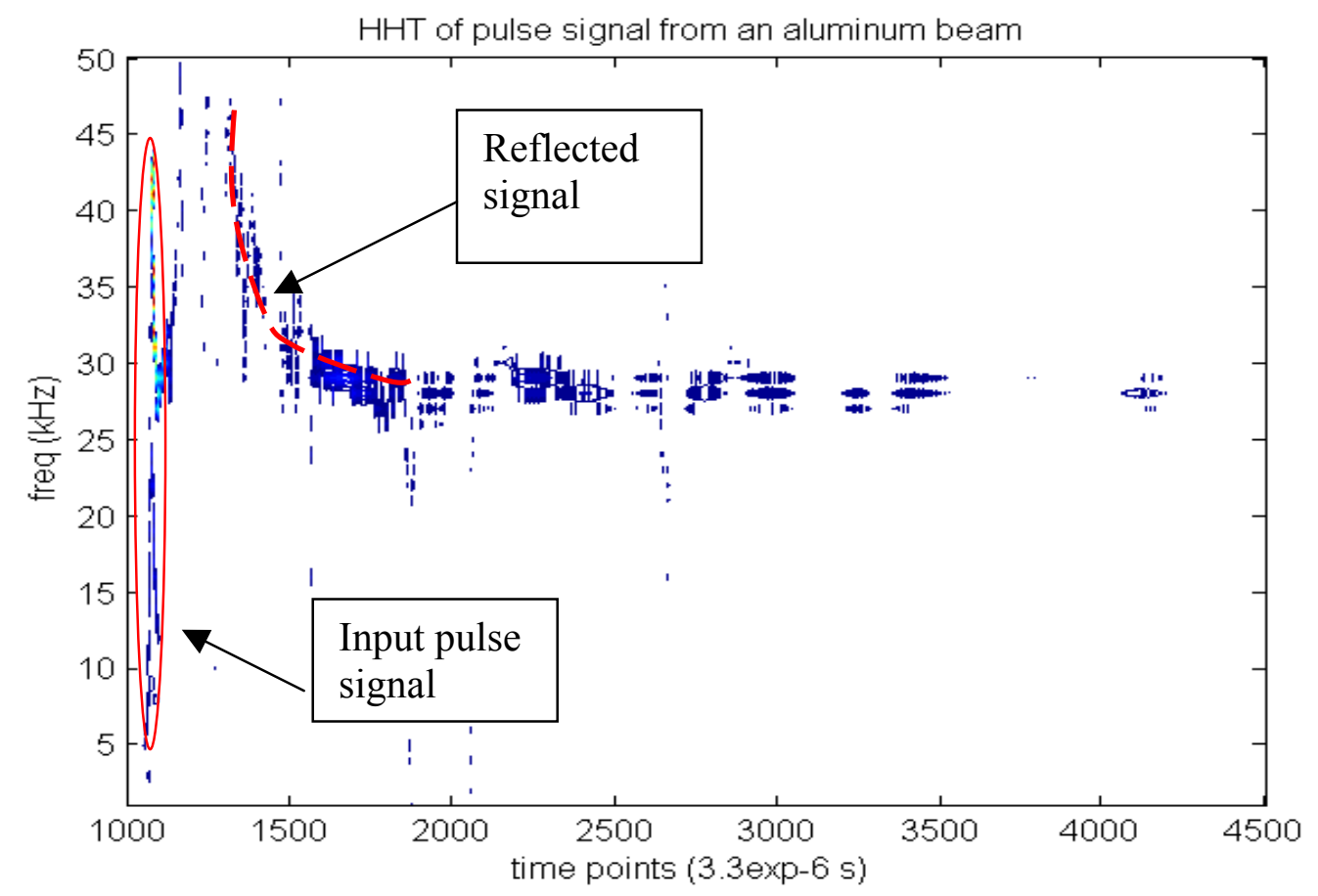

Figure 6.17. HHT of a pulse signal from an aluminum plate.

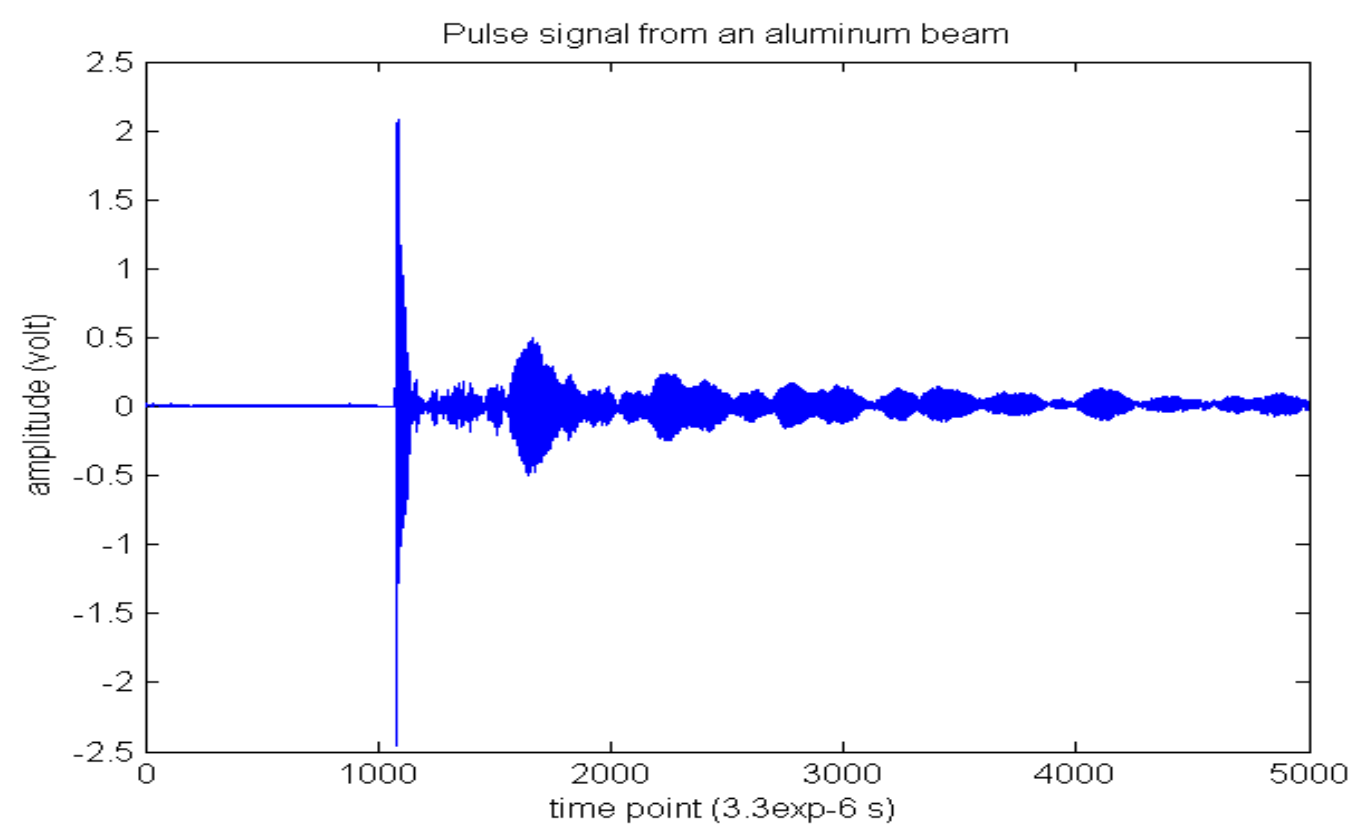

Figure 6.18. A pulse signal from an aluminum plate. 


\section{INTEGRITY MONITORING OF METAL TUBING}

\subsection{Structural Flaw Evaluation in Air}

After mode separation, the Lamb wave signals from a brass tube are passed through a moving window with an optimal width. The properties around local peaks are then calculated as defined in Section 4. Five structural conditions are tested for a three feet long brass tube and are listed in Table 7.1. The results of analysis from moving windows are plotted in the following figures. Figures 7.1 - 7.4 show the results with 14 $\mathrm{kHz}$ active wave propagating from right to left. The anti-symmetric mode waves are used in these figures. It is clear that the structural flaws cause the decrease of the amplitude of local peaks, while the variance of windowed signals increases due to the scattering of structural discontinuity. Figures 7.1 and 7.2 demonstrate the left part and right part of the gravity centers of windowed signals shifting away from the middle point of the window. It is noticed that the left part weight center of the first local peak does not change because the left part of the first peak is not affected by the scattering of Lamb waves.

The severity of structural defects could be estimated using these figures. The size of flaw is roughly proportional to the decrease of amplitude. The variance doesn't change much for the first local peak under different conditions, but the difference increases with time because of wave spread during propagation. The larger the flaw size, the bigger is the difference between normal and flaw curves. All of these could be used for the evaluation of structural conditions. 
Table 7.1. Five structural conditions tested for a brass tube (3 feet long) in the air

\begin{tabular}{|c|c|c|c|}
\hline Structural conditions & $\begin{array}{l}\text { Activation } \\
\text { frequency }\end{array}$ & $\begin{array}{c}\text { Wave } \\
\text { propagation } \\
\text { direction }\end{array}$ & Active sensor \\
\hline 1. Normal & $\begin{array}{l}13 \mathrm{kHz}, 14 \mathrm{kHz} \\
15 \mathrm{kHz}, 16 \mathrm{kHz}\end{array}$ & $\begin{array}{l}\text { Left to right, right } \\
\text { to left }\end{array}$ & $\begin{array}{l}\text { Left-up, left-bottom, } \\
\text { right-up, right - } \\
\text { bottom }\end{array}$ \\
\hline 2. Half hole $(\mathrm{d}=0.6 \mathrm{~mm})$ & $\begin{array}{l}13 \mathrm{kHz}, 14 \mathrm{kHz} \\
15 \mathrm{kHz}, 16 \mathrm{kHz}\end{array}$ & $\begin{array}{l}\text { Left to right, right } \\
\text { to left }\end{array}$ & Same as above \\
\hline 2. Half hole $(\mathrm{d}=1.8 \mathrm{~mm})$ & $\begin{array}{l}13 \mathrm{kHz}, 14 \mathrm{kHz}, \\
15 \mathrm{kHz}, 16 \mathrm{kHz}\end{array}$ & $\begin{array}{l}\text { Left to right, right } \\
\text { to left }\end{array}$ & Same as above \\
\hline $\begin{array}{l}\text { 3. Through hole }(\mathrm{d}=1.8 \text { upper, } \\
\mathrm{d}=0.6 \mathrm{~mm} \text { bottom })\end{array}$ & $\begin{array}{l}13 \mathrm{kHz}, 14 \mathrm{kHz}, \\
15 \mathrm{kHz}, 16 \mathrm{kHz}\end{array}$ & $\begin{array}{l}\text { Left to right, right } \\
\text { to left }\end{array}$ & Same as above \\
\hline 4. $\operatorname{Notch}(1.5 \mathrm{~mm} \times 8 \mathrm{~mm})$ & $\begin{array}{l}13 \mathrm{kHz}, 14 \mathrm{kHz}, \\
15 \mathrm{kHz}, 16 \mathrm{kHz}\end{array}$ & $\begin{array}{l}\text { Left to right, right } \\
\text { to left }\end{array}$ & Same as above \\
\hline
\end{tabular}

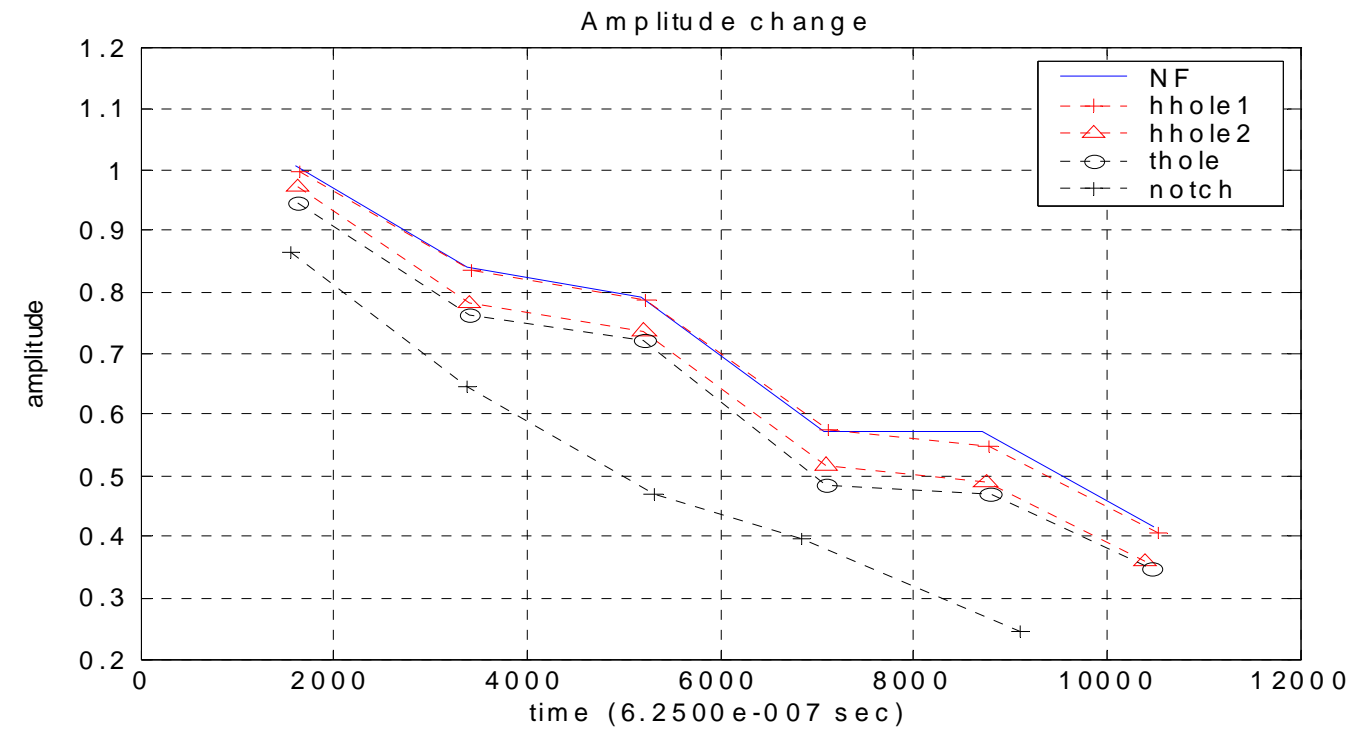

Figure 7.1. Amplitude change of local peaks of anti-symmetric mode signals, propagating from the right to the left, with $14 \mathrm{kHz}$ input frequency. 


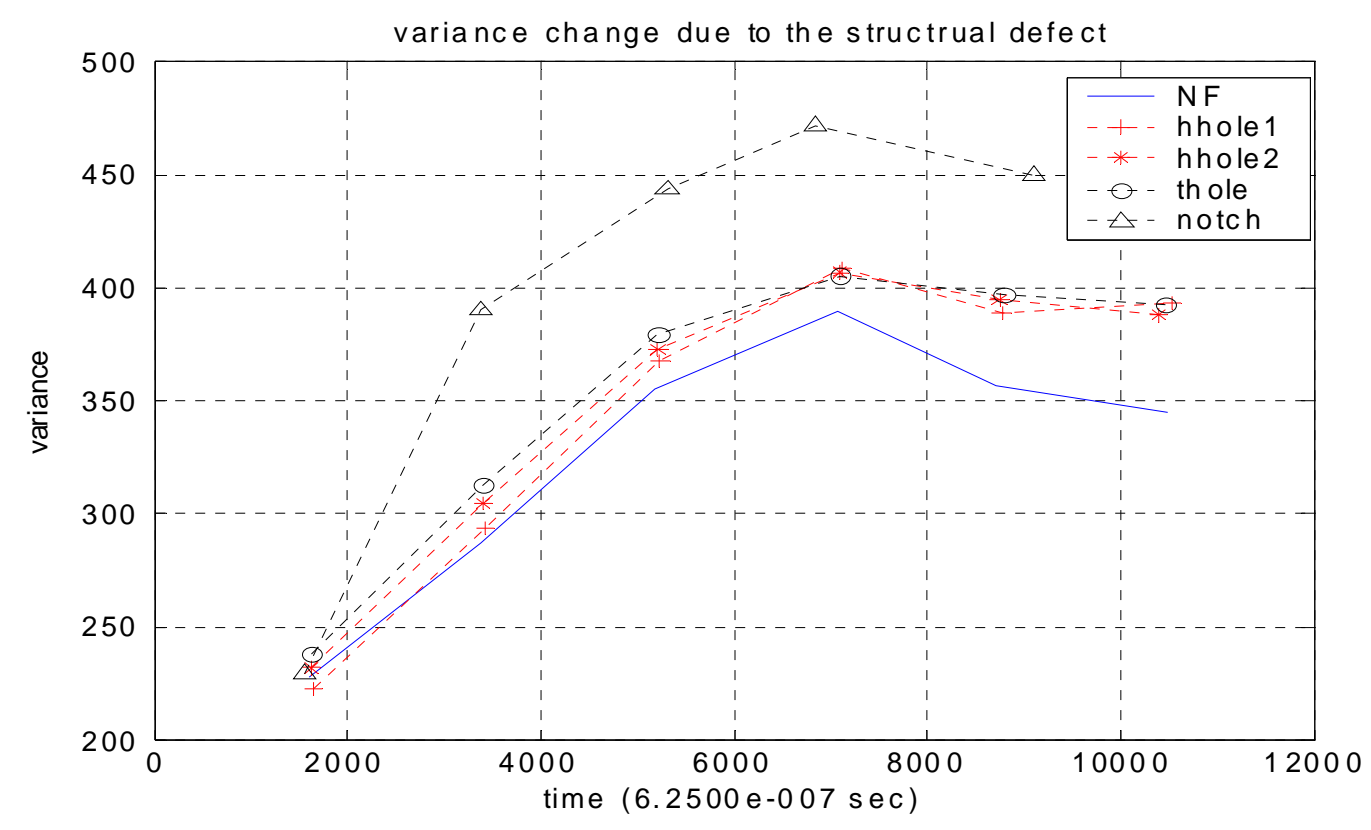

Figure 7.2. Change of variance of local peaks from anti-symmetric mode signals propagating from the right to the left end, with input frequency $14 \mathrm{kHz}$.

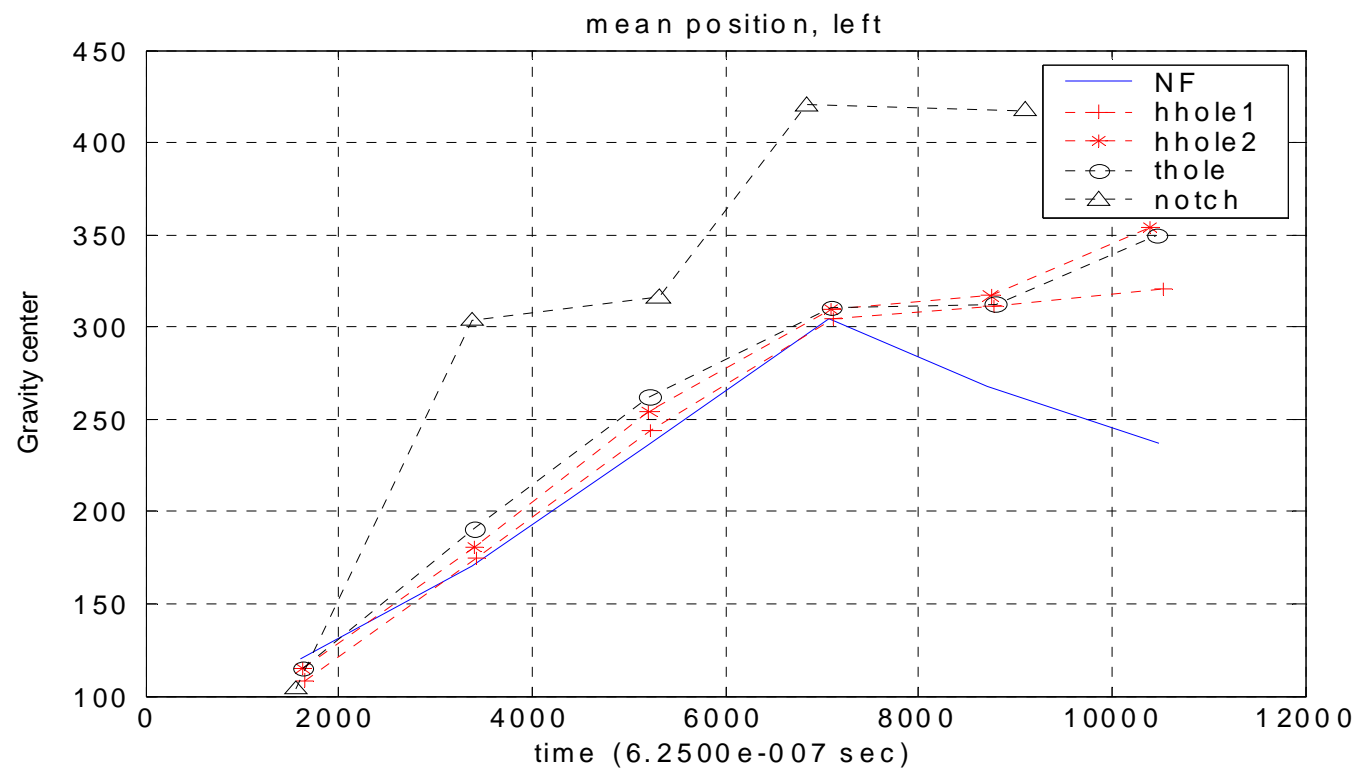

Figure 7.3. Change of left part weight center of local peaks from anti-symmetric mode signals propagating from the right to the left end, input frequency $14 \mathrm{kHz}$. 


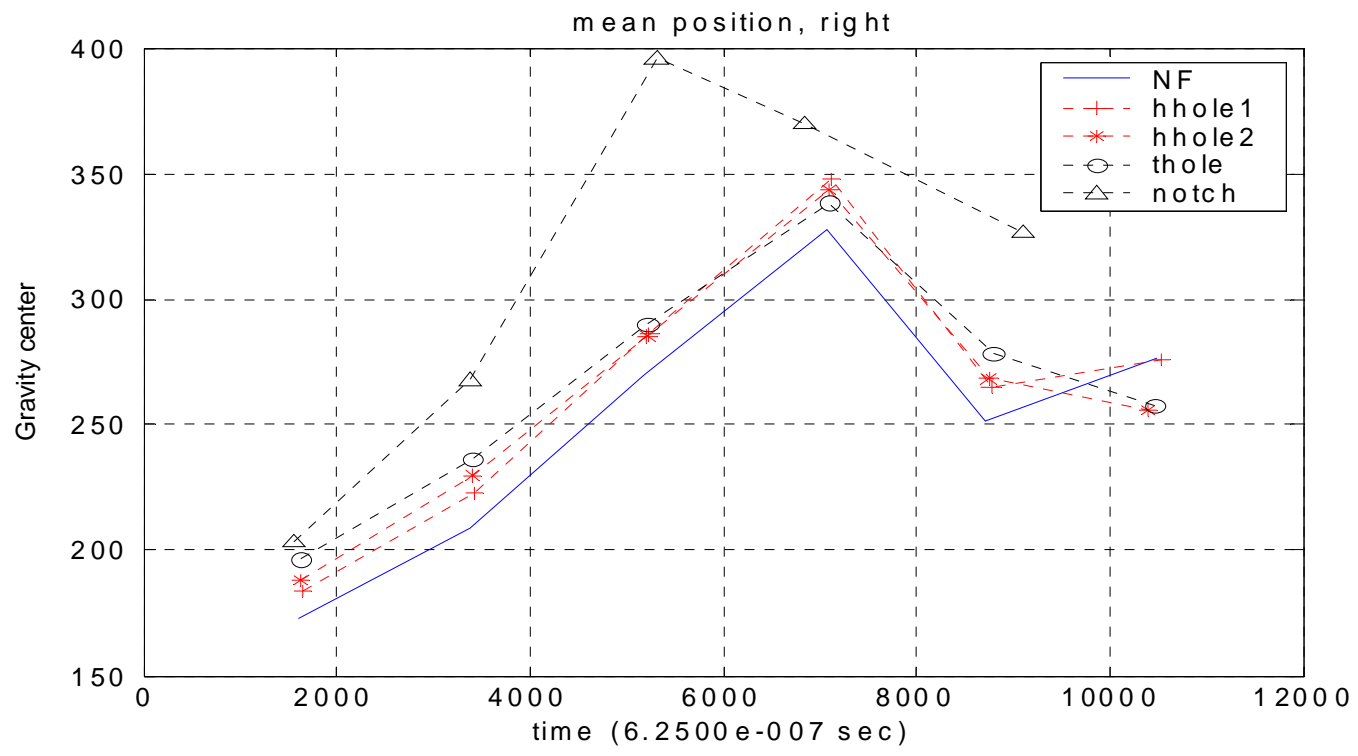

Figure 7.4. Change of right part weight center of local peaks from anti-symmetric mode signals propagating from the right to the left end, with input frequency $14 \mathrm{kHz}$.

Compared with the anti-symmetric mode, the symmetric mode waves corresponding to the anti-symmetric signals used in Figures $7.1-7.4$ are not sensitive to structural changes. As shown in Figures 7.5 - 7.8, symmetric waves cannot systematically indicate the existence of structural anomaly as do anti-symmetric waves. The reason why the symmetric Lamb waves are not sensitive to structural changes is discussed below.

In order to check the robustness and sensitivity of the method using antisymmetric mode Lamb waves for structural diagnostics, different activation frequencies and locations of active sensors are tested. Figures A.1 through A.4 in appendix II show the results with the activation wave propagating from left to right, and the input frequency is still $14 \mathrm{kHz}$. We find similar result as shown in Figures 7.1 - 7.4, except in the plot of the gravity center of the left part of windowed signals the normal curve is distorted. This is the reason we need to repeat the experiment using different frequencies and active sensor locations before a final decision could be made. 
The input frequency is then changed to $13 \mathrm{kHz}$, with the active wave propagating from left to right. The results are shown in Figures A.5 through A.8. We find very similar results as in Figures 7.1 - 7.4. This verifies the validity of the moving window technique in evaluating the structural integrity using anti-symmetric signals.

The reason why the symmetrical is mode not sensitive to the structural flaw is that the reflections from structural flaws are generally axially non-symmetrical no matter what are the input Lamb wave signals. Hence most features of the structural changes would be displayed in flexural mode signals after the mode separation. This also demonstrates the importance of the mode separation for tube health monitoring since this process filters out axial symmetrical signals not representing the change of tubular structures.

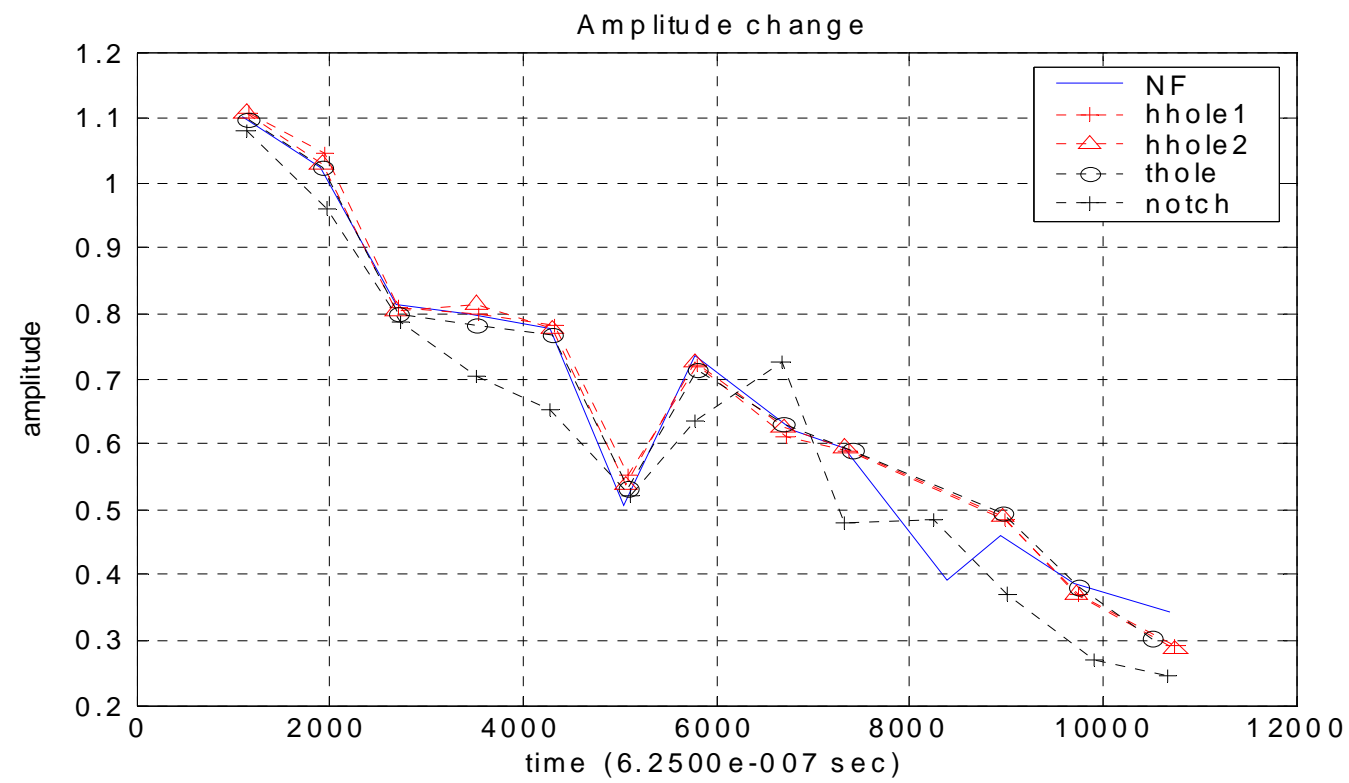

Figure 7.5. Amplitude change of local peaks of symmetric mode signals propagating from right to left, input frequency $14 \mathrm{kHz}$. 


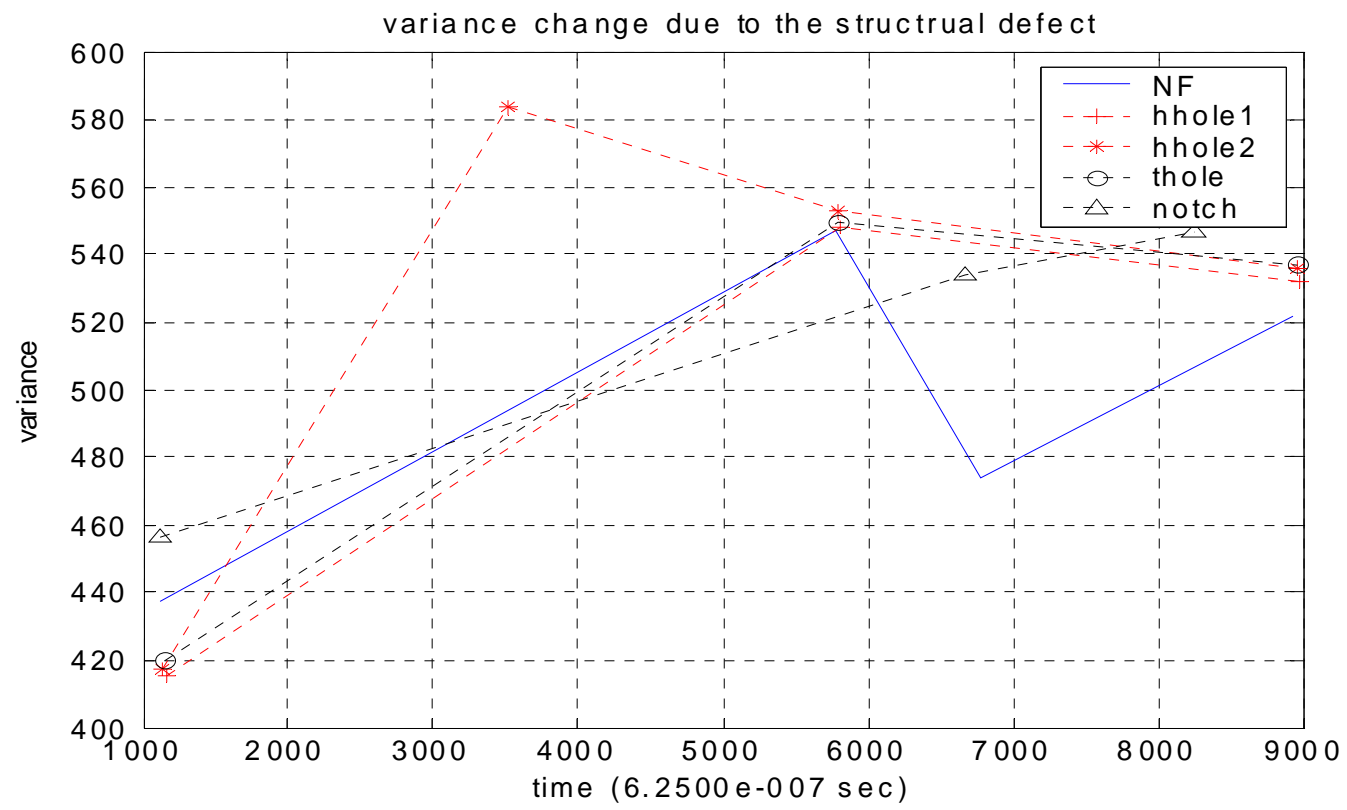

Figure 7.6. Change of variance of local peaks of symmetric mode signals, propagating from right to left, input frequency $14 \mathrm{kHz}$.

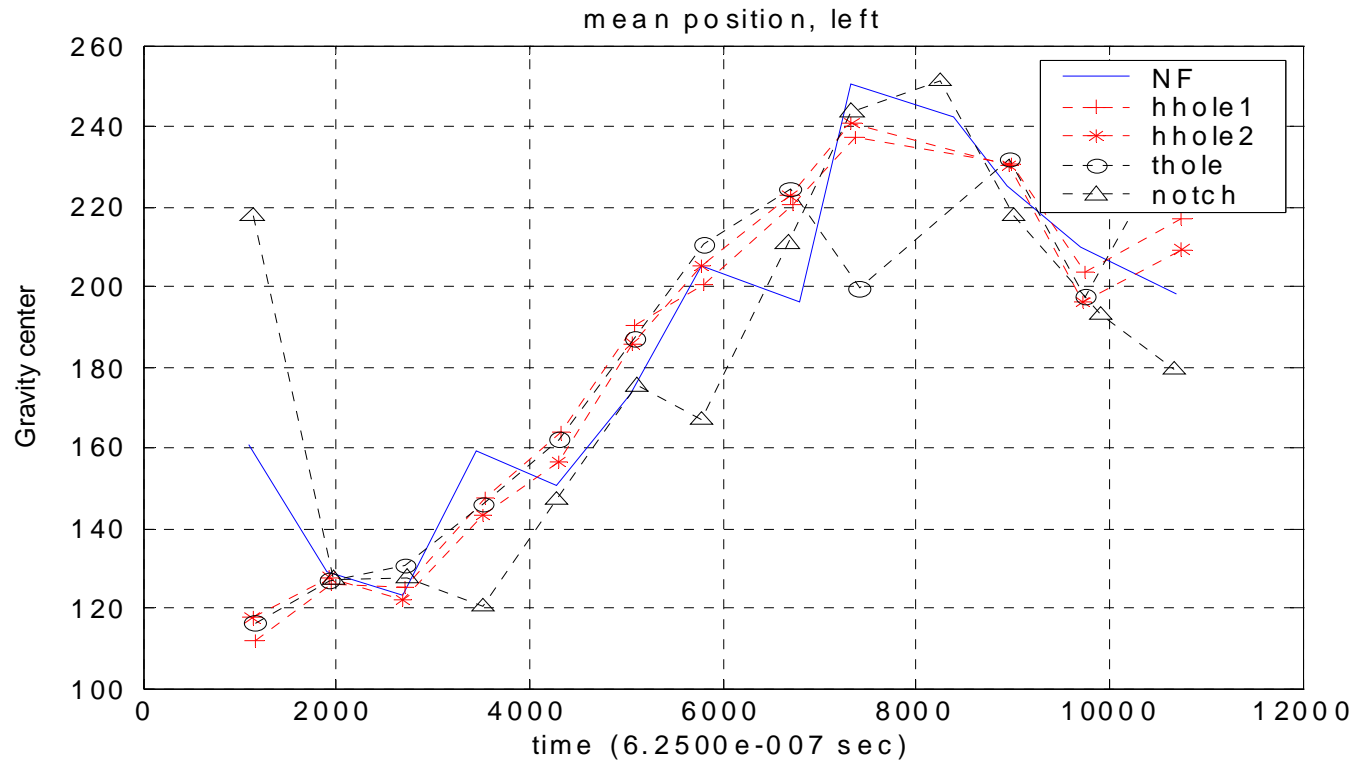

Figure 7.7. Change of left part weight center of local peaks of symmetric mode signals propagating from right to, input frequency $14 \mathrm{kHz}$. 


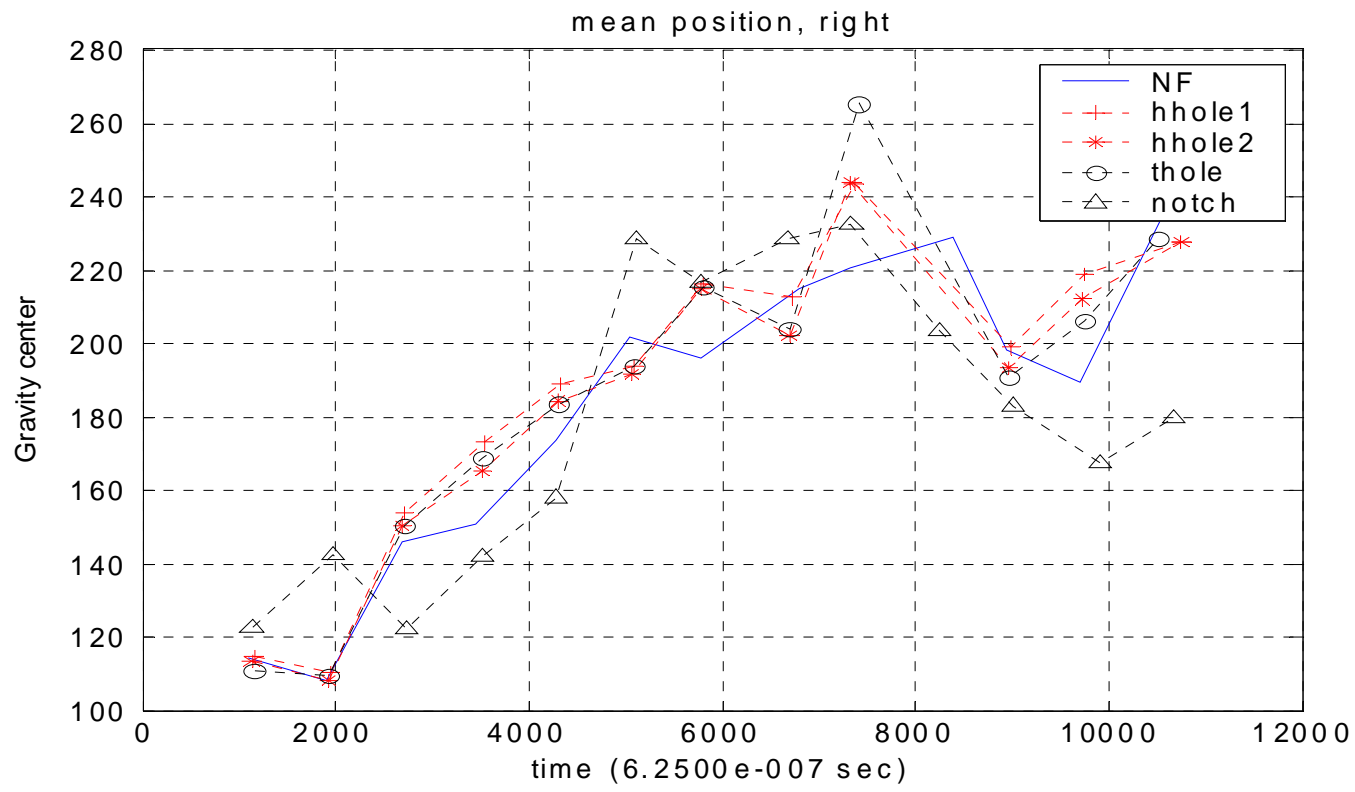

Figure 7.8. Change of right part weight center of local peaks of symmetric mode signals, propagating from right to left, input frequency $14 \mathrm{kHz}$. 


\subsection{Structural Flaw Evaluation in Water}

In practice, we are interested in acoustic propagation along the tubing immersed in water because our final objective of this research is detecting structural flaws in the SG U-tubes. A summary of experiments with the specimen submerged in water is given in Table 7.2. Transient signal processing methods, similar to those used in experiments performed in air, are implemented. The data processing results are illustrated in Figures 7.9 - 7.12. Obviously the moving window method is also very effective for the brass tube examination performed in the water. The different structural conditions are clearly separated in the four representative features extracted. The difference observed in Figure 7.9 about the local peak amplitude is that the local peak energy drops more quickly than what was observed for tests in air and shown in Figure 7.1. Further comparison will be given in the next section using the experimental data of another specimen tested both in air and in water.

An abnormal data set in Figure 7.1 is the data from the notch condition, whose amplitude is larger than the normal one. This is due to the error in the experimental procedure. Unlike the experiments in the air, we could not create a designed flaw on site in the water. The flaw was created outside the water, and the specimen was then put back in the water tank. Although we tried our best to keep the boundary conditions unchanged, it could not be perfectly duplicated. However, the other three features are not so sensitive to the boundary condition change as demonstrated in Figures 7.10 - 7.12. The structural flaws cause significant changes in the local peak spread and the local gravity centers.

We could conclude that the moving window technique worked excellently in the water without the effect of noise. 
Table 7.2. Five conditions tested for a brass tube (2 feet long) in water (\#10)

\begin{tabular}{|c|l|l|l|}
\hline Structural conditions in Water & $\begin{array}{l}\text { Activation } \\
\text { frequency }\end{array}$ & $\begin{array}{l}\text { Wave propagation } \\
\text { direction }\end{array}$ & $\begin{array}{l}\text { Sampling } \\
\text { frequency }\end{array}$ \\
\hline 2. Half hole $(\mathrm{d}=0.6 \mathrm{~mm})$ & $\begin{array}{l}13 \mathrm{kHz}, 14 \mathrm{kHz}, \\
15 \mathrm{kHz}, 16 \mathrm{kHz}\end{array}$ & $\begin{array}{l}\text { Left to right, right } \\
\text { to left }\end{array}$ & $1.6 \mathrm{MHz}$ \\
& $\begin{array}{c}13 \mathrm{kHz}, 14 \mathrm{kHz}, \\
15 \mathrm{kHz}, 16 \mathrm{kHz}\end{array}$ & $\begin{array}{l}\text { Left to right, right } \\
\text { to left }\end{array}$ & $1.6 \mathrm{MHz}$ \\
\hline 2. Half hole $(\mathrm{d}=1.8 \mathrm{~mm})$ & $13 \mathrm{kHz}, 14 \mathrm{kHz}$, & $\begin{array}{l}\text { Left to right, right } \\
\text { to left }\end{array}$ & $1.6 \mathrm{MHz}$ \\
\hline $\begin{array}{c}15 \mathrm{kHz}, 16 \mathrm{kHz} \\
\text { 3hrough hole }(\mathrm{d}=1.8 \text { upper, } \\
\mathrm{d}=0.6 \mathrm{~mm} \text { bottom })\end{array}$ & $\begin{array}{c}13 \mathrm{kHz}, 14 \mathrm{kHz}, \\
\text { 4. Notch }(1.5 \mathrm{~mm} \times 8 \mathrm{~mm})\end{array}$ & $\begin{array}{l}\text { Left to right, right } \\
\text { to left }\end{array}$ & $1.6 \mathrm{MHz}$ \\
\hline 2. Half hole $(\mathrm{d}=0.6 \mathrm{~mm})$ & $13 \mathrm{kHz}, 16 \mathrm{kHz}$ & $\begin{array}{l}\text { Left to right, right } \\
\text { to left }\end{array}$ & $1.6 \mathrm{MHz}$ \\
& $15 \mathrm{kHz}, 14 \mathrm{kHz}, 16 \mathrm{kHz}$ & \\
\hline
\end{tabular}




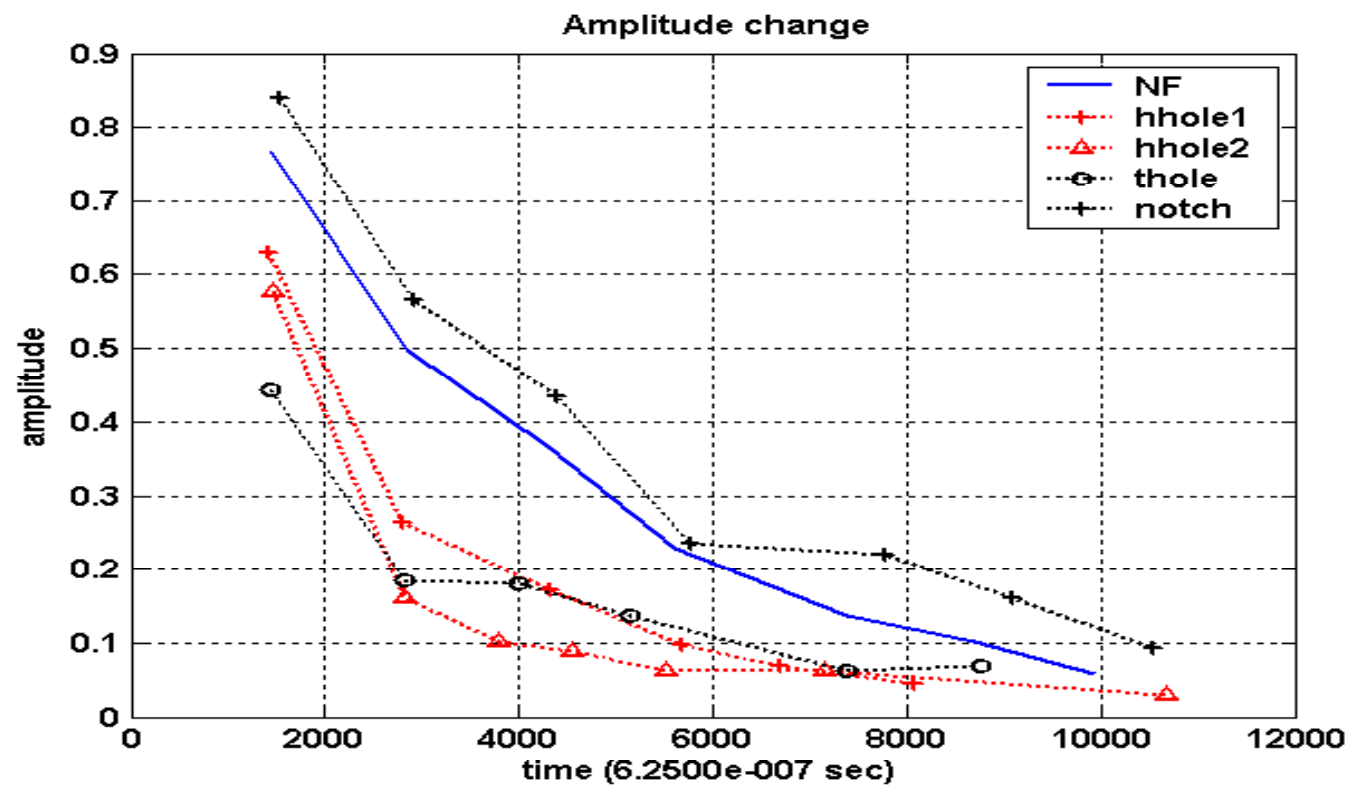

Figure 7.9. Amplitude change of local peaks of anti-symmetric mode signals in the water, propagating from left to right, with $13 \mathrm{kHz}$ input frequency.

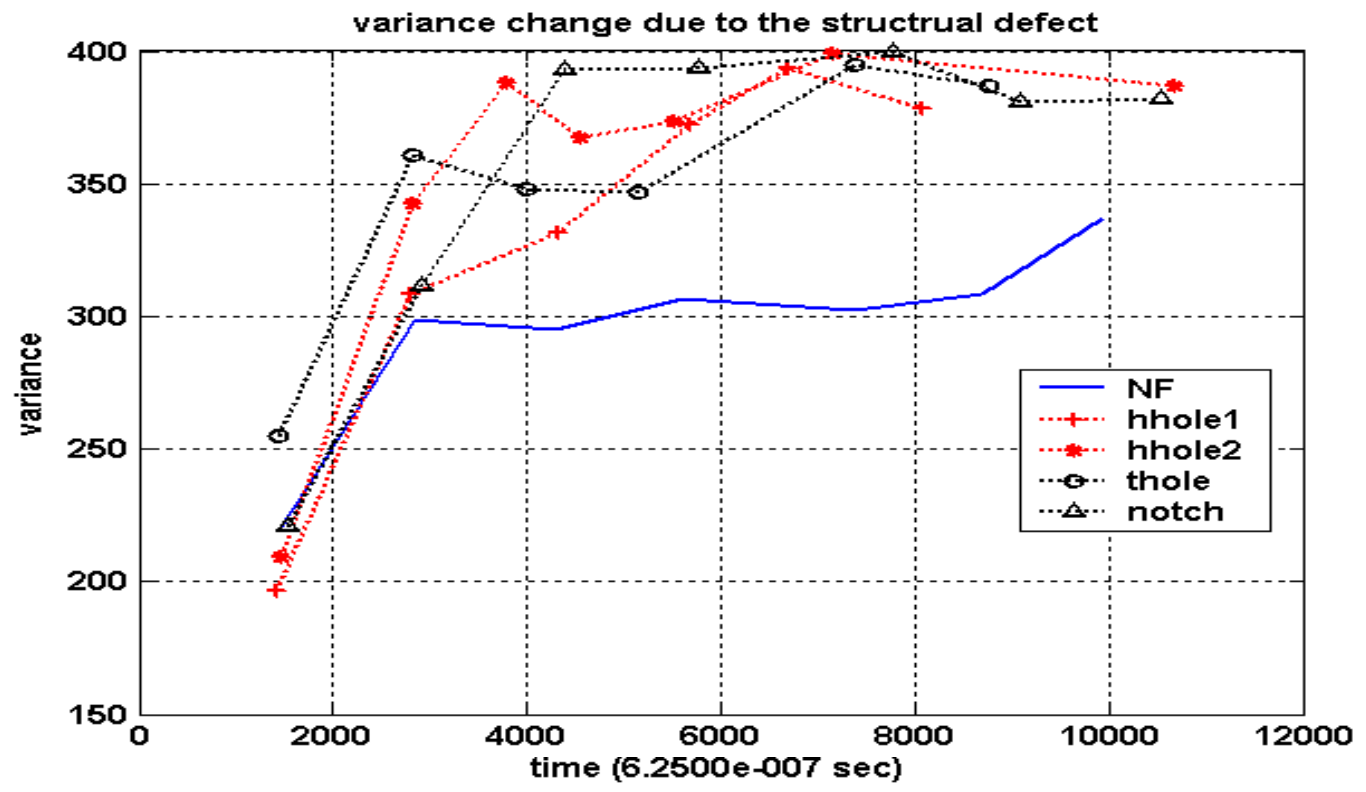

Figure 7.10. Variance change of local peaks of anti-symmetric mode signals in the water, propagating from left to right, with $13 \mathrm{kHz}$ input frequency. 


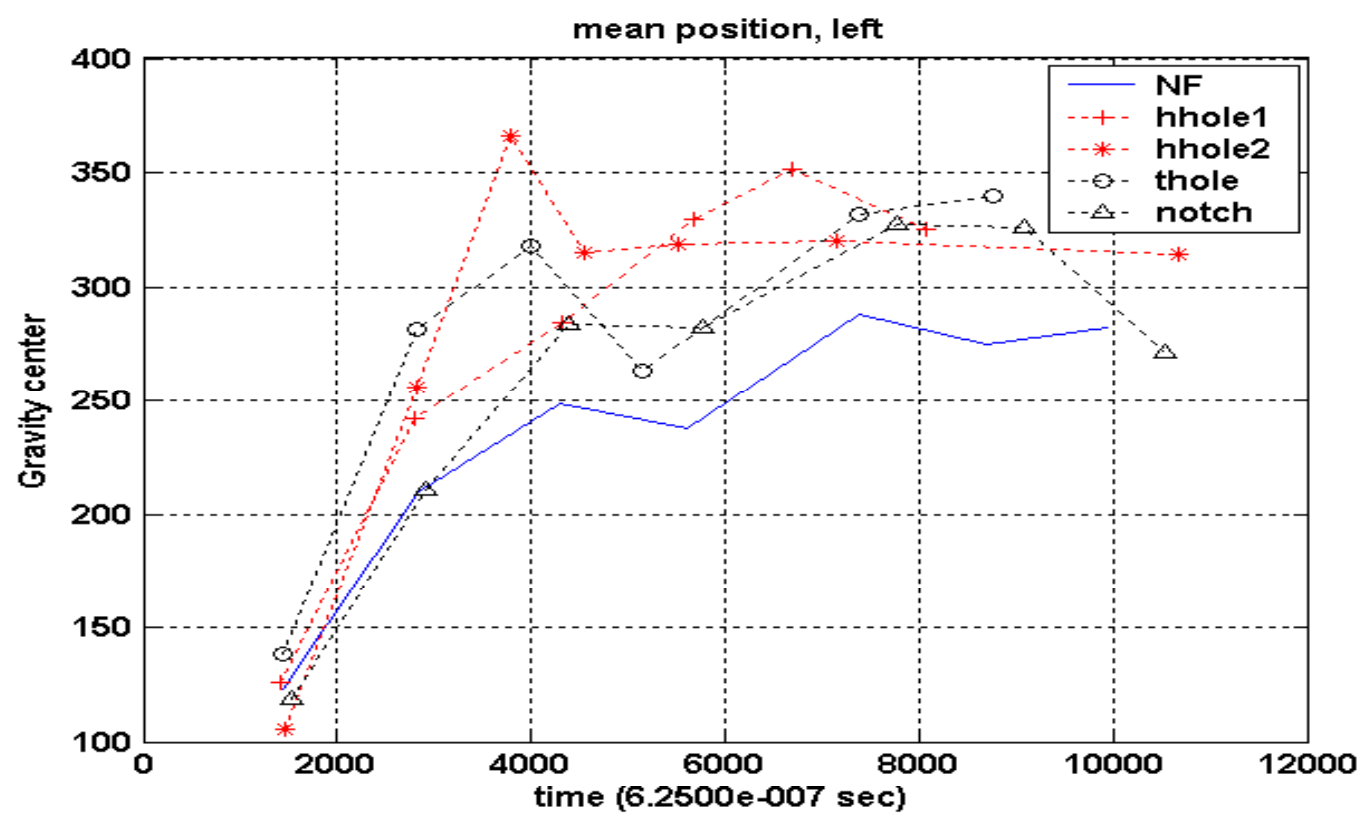

Figure 7.11. Left weight center change of local peaks of anti-symmetric mode signals in the water, propagating from left to right, with $13 \mathrm{kHz}$ input frequency.

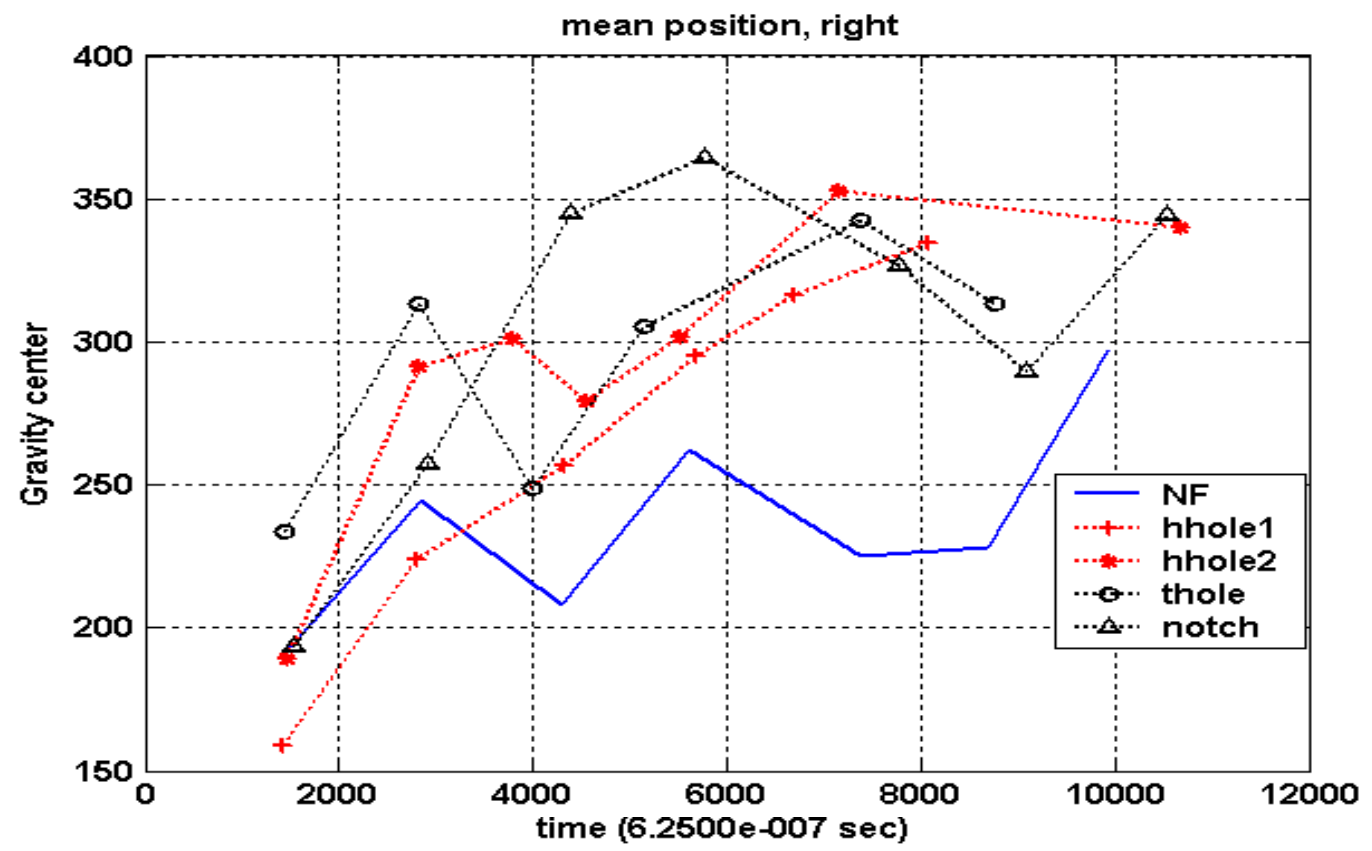

Figure 7.12. Right weight center change of local peaks of anti-symmetric mode signals in the water, propagating from left to right, with $13 \mathrm{kHz}$ input frequency. 


\subsection{Comparison of Structural Flaw Evaluation in Air and in Water}

As we mentioned above, we are interested to know the difference of Lamb wave signals in a brass tube in air and in water. Therefore, another tube was tested in both air and in water. The experiments performed are summarized in Table 7.3.

Moving window method was again used for analysis, however, different window size was used because the sampling frequency was $300 \mathrm{kHz}$, much lower than $1.6 \mathrm{MHz}$ used in the experiments listed in Tables 7.1 and 7.2. The results of analysis are illustrated in Figures $7.13-7.16$. It is obvious that the moving window method works excellently even for the case of low sampling frequency.

The results demonstrate that the effect of water on the brass tubes causes more energy leakage from tube to the media around, therefore the amplitude of local peaks of tube waves is lower in the water than in the air. On the other hand, the variance of local peaks in the water is larger than that in the air. The change of gravity centers in water is similar to that in the air. In summary, the moving window method is still an effective algorithm for the analysis of acoustic waves from the tubes immersed in water.

Table 7.3. Six conditions tested for a brass tube in both air and water (\#4)

\begin{tabular}{|l|l|l|l|}
\hline Structural conditions & Activation frequency & $\begin{array}{l}\text { Wave propagation } \\
\text { direction }\end{array}$ & $\begin{array}{l}\text { Sampling } \\
\text { frequency }\end{array}$ \\
\hline 1. Normal, in air & $\begin{array}{l}16 \mathrm{kHz}, 17 \mathrm{kHz}, 18 \mathrm{kHz}, \\
19 \mathrm{kHz}\end{array}$ & $\begin{array}{l}\text { Left to right, right to } \\
\text { left }\end{array}$ & $300 \mathrm{kHz}$ \\
\hline $\begin{array}{l}\text { 2. Half hole }(\mathrm{d}=0.6 \mathrm{~mm}), \text { in } \\
\text { air }\end{array}$ & $\begin{array}{l}16 \mathrm{kHz}, 17 \mathrm{kHz}, 18 \mathrm{kHz}, \\
19 \mathrm{kHz}\end{array}$ & $\begin{array}{l}\text { Left to right, right to } \\
\text { left }\end{array}$ & $300 \mathrm{kHz}$ \\
\hline $\begin{array}{l}\text { 3. Through hole } \\
\text { (d=0.6mm), in air }\end{array}$ & $\begin{array}{l}16 \mathrm{kHz}, 17 \mathrm{kHz}, 18 \mathrm{kHz}, \\
19 \mathrm{kHz}\end{array}$ & $\begin{array}{l}\text { Left to right, right to } \\
\text { left }\end{array}$ & $300 \mathrm{kHz}$ \\
\hline $\begin{array}{l}\text { 4. Normal, in water } \\
16 \mathrm{kHz}, 17 \mathrm{kHz}, 18 \mathrm{kHz}, \\
19 \mathrm{kHz}\end{array}$ & $\begin{array}{l}\text { Left to right, right to } \\
\text { left }\end{array}$ & $300 \mathrm{kHz}$ \\
\hline $\begin{array}{l}\text { 5. Half hole }(\mathrm{d}=0.6 \mathrm{~mm}), \text { in } \\
\text { water }\end{array}$ & $\begin{array}{l}16 \mathrm{kHz}, 17 \mathrm{kHz}, 18 \mathrm{kHz}, \\
19 \mathrm{kHz}\end{array}$ & $\begin{array}{l}\text { Left to right, right to } \\
\text { left }\end{array}$ & $300 \mathrm{kHz}$ \\
\hline $\begin{array}{l}\text { 6. Through hole }(\mathrm{d}=0.6), \\
\text { in water }\end{array}$ & $\begin{array}{l}16 \mathrm{kHz}, 17 \mathrm{kHz}, 18 \mathrm{kHz}, \\
19 \mathrm{kHz}\end{array}$ & $\begin{array}{l}\text { Left to right, right to } \\
\text { left }\end{array}$ & $300 \mathrm{kHz}$ \\
\hline
\end{tabular}




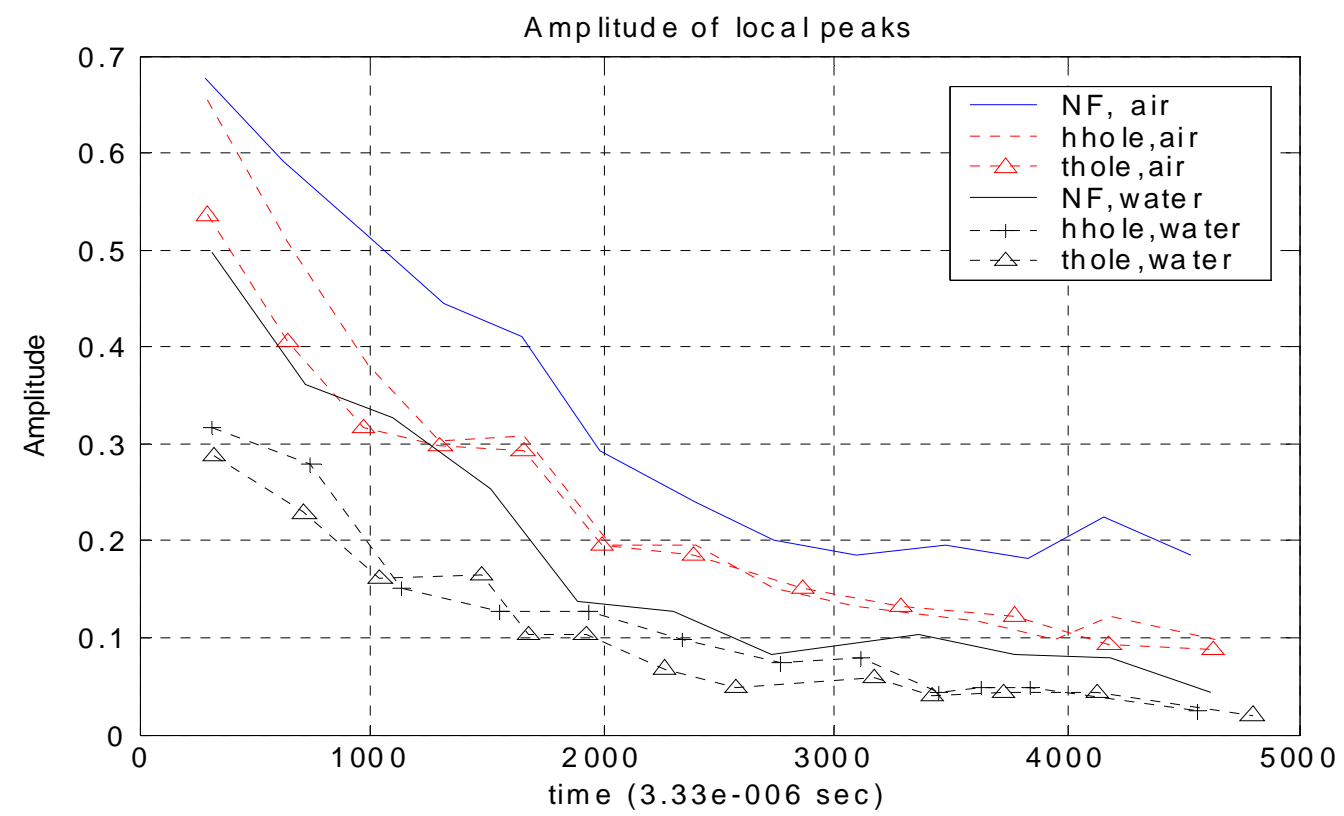

Figure 7.13. Amplitude change of local peaks from anti-symmetric mode signals, propagating from the left to the right, with input frequency $16 \mathrm{kHz}$.

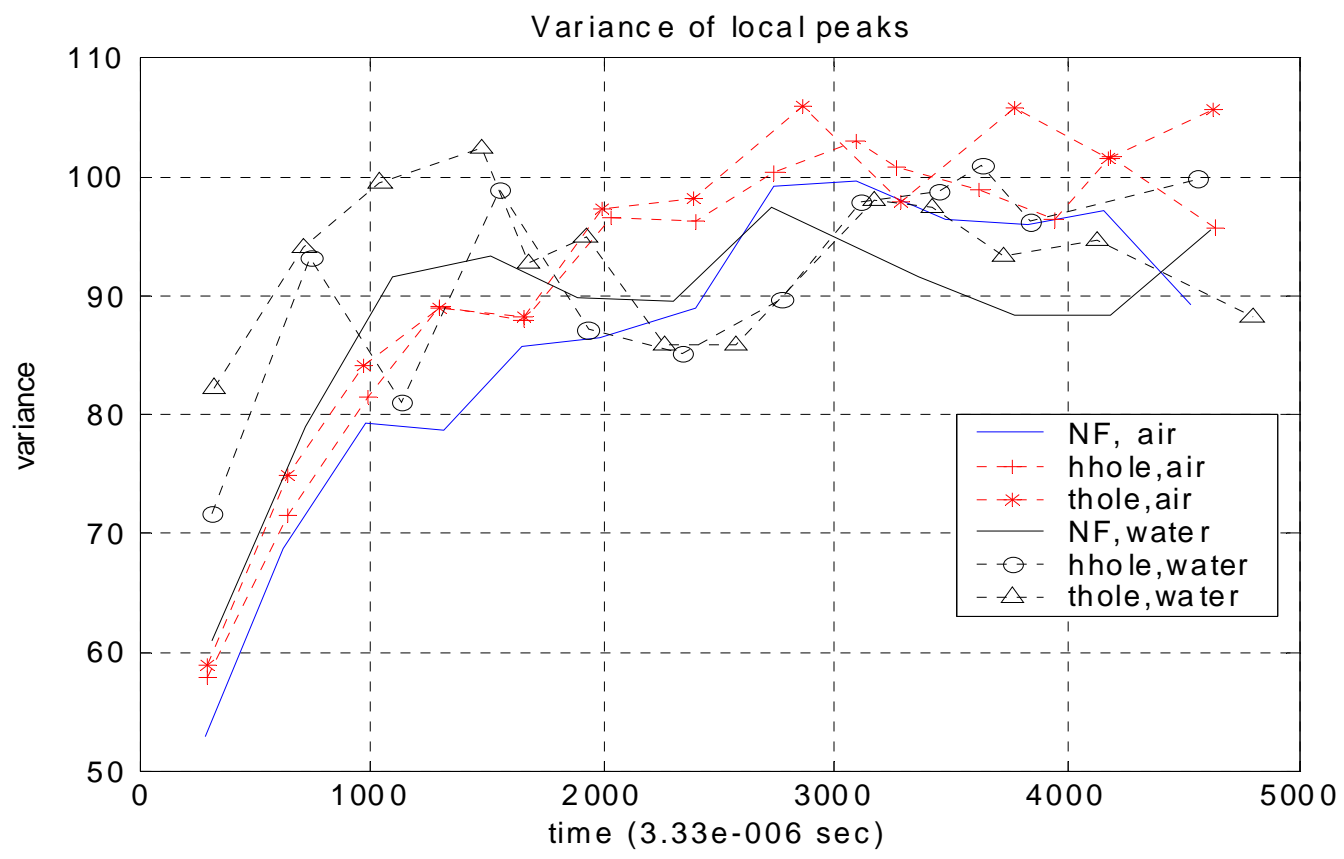

Figure 7.14. Change of variance of local peaks from anti-symmetric mode signals, propagating from the left to the right, with input frequency $16 \mathrm{kHz}$. 


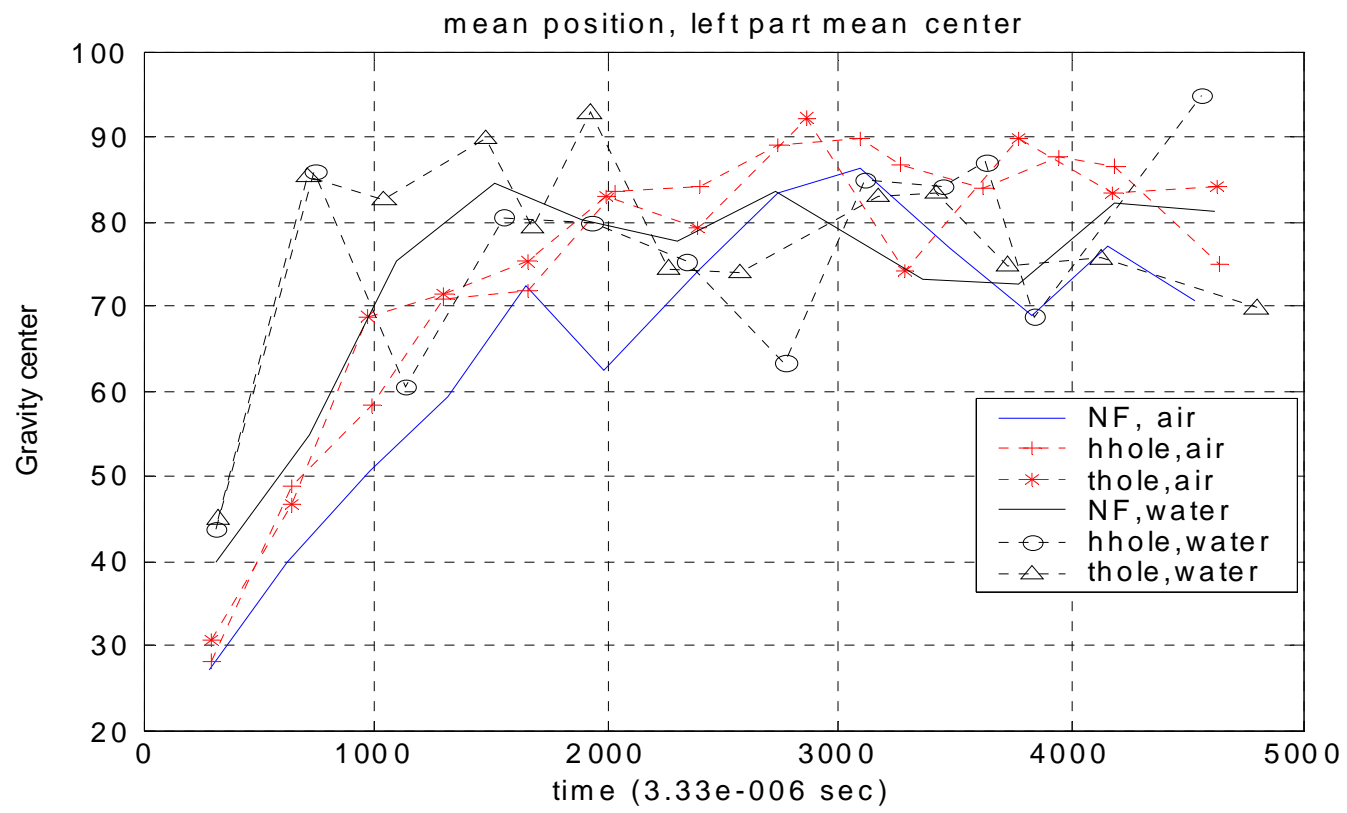

Figure 7.15. The change of left part weight center of local peaks from anti-symmetric mode signals, propagating from the left to the right, with input frequency $16 \mathrm{kHz}$.

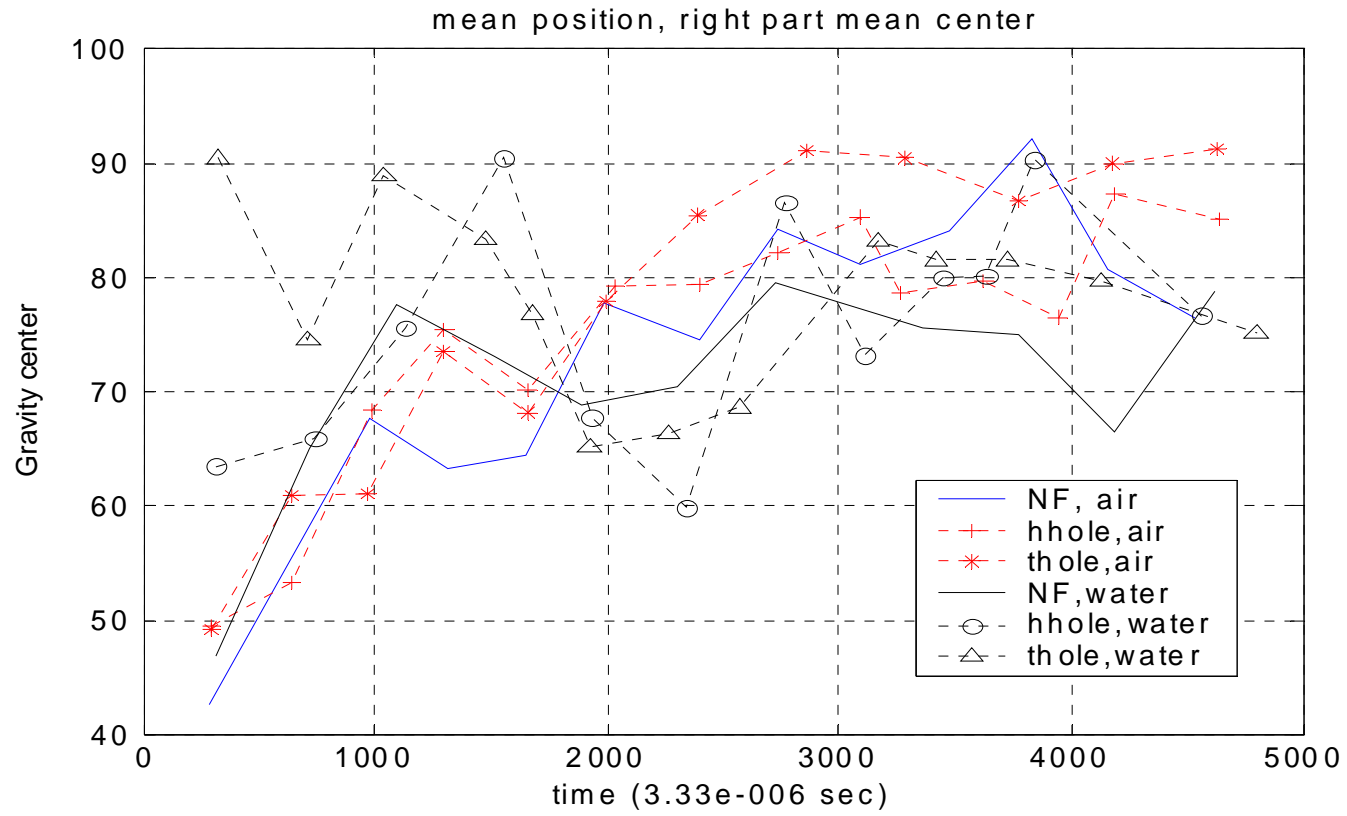

Figure 7.16. The change of right part weight center of local peaks from anti-symmetric mode signals, propagating from the left to the right, with input frequency $16 \mathrm{kHz}$. 


\subsection{Estimation of Defect Location}

\subsubsection{Flaw localization for brass tube in air}

Defect localization for tubing is not as simple as for a plate-like structure because the complexity of the scattering phenomena of tubular acoustic waves. The reflection of acoustic waves in a tube is weak and widely spread. Therefore no obvious reflected peaks are found between local peaks in the raw signals. The methods used in aluminum plate analysis such as HHT and extrema extraction cannot generate satisfactory results. So a new method named zooming windows is introduced to estimate the flaw position in tube-like structures. The zooming windows are applied onto the first two local peaks in the separated anti-symmetric waves. The right weight center of the first local peak is calculated and the left part weight center of the second peak is calculated as the window zooming around its center.

As illustrated in Figure 7.17 below, the curves of left or right part weight centers should diverge due to the effect of structural reflection. The divergent point indicates the approximate position of the defect. Therefore we are able to tell roughly where the flaw exists.

Figures 7.18 - 7.21 demonstrate the effectiveness of the zooming window. In Figures 7.18 and 7.19, we notice that the divergent point for the first peak is earlier than for the second local peak. This indicates that the defect here is near the receiving sensors. In fact the defect here is located at about one-third of tube length to the receiving sensors. The observation of Figures 7.20 and 7.21 indicate that the diverging point for the first peak is later than the second peak. So we can tell the defect is far away from the passive sensors, and we know that the defect is in fact located at about two-thirds of tube length to the passive sensors. 


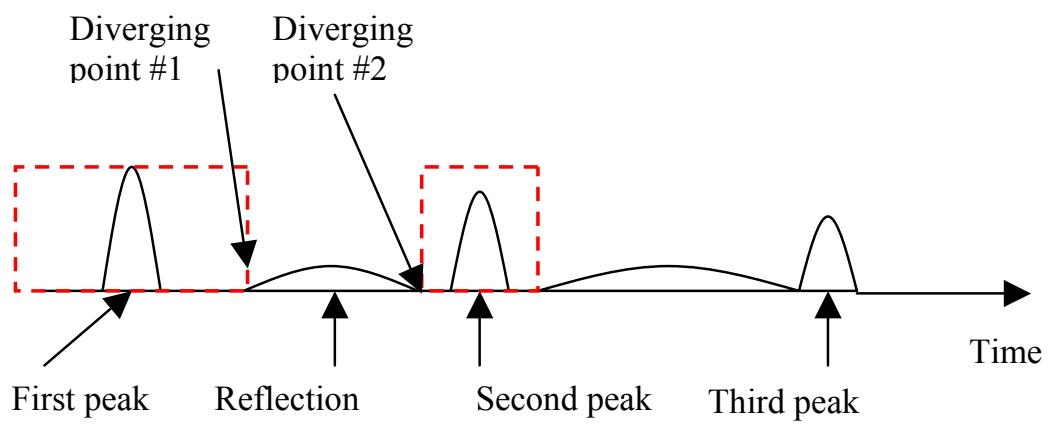

Figure 7.17. The zooming windows and the diverging points.

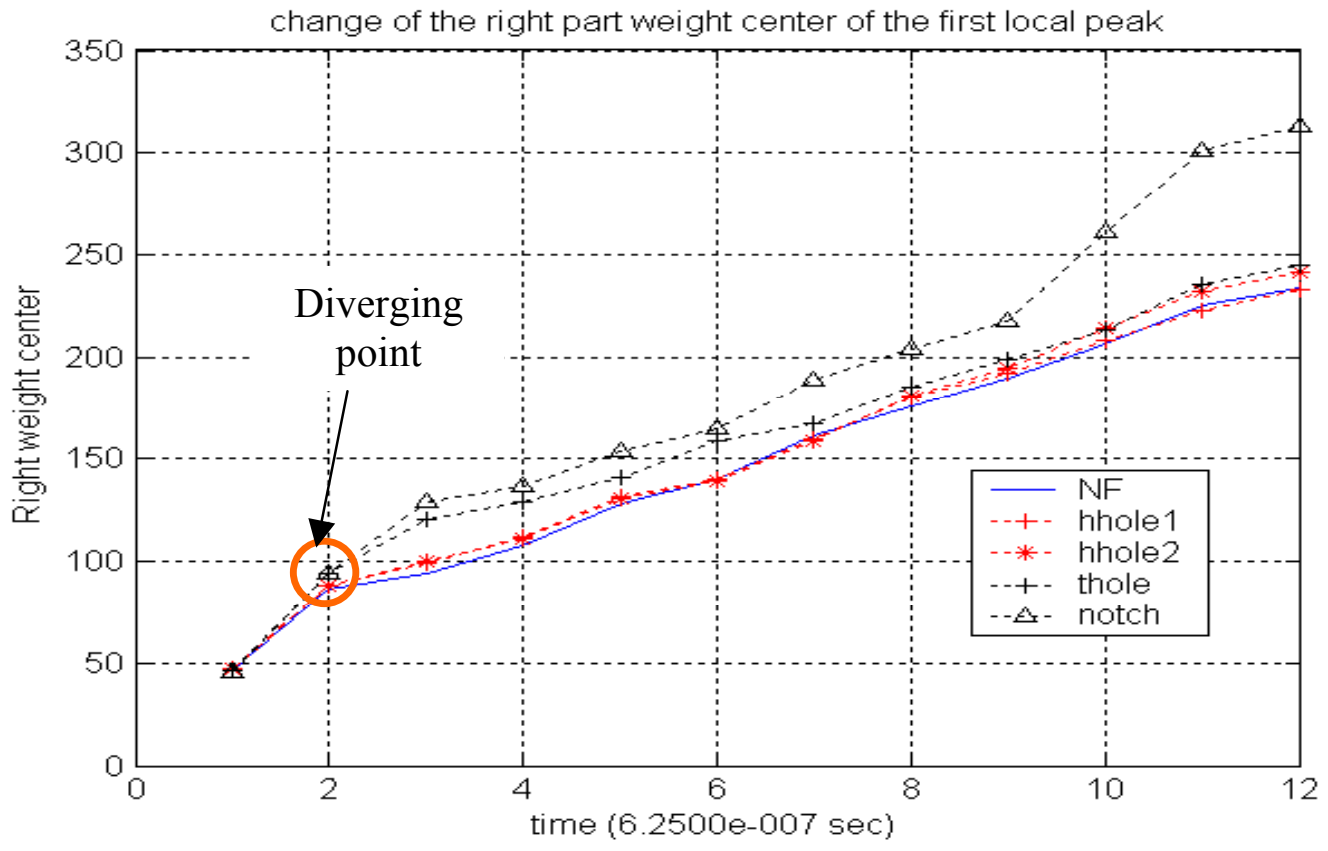

Figure 7.18. The change of right part weight center of the first local peak as the zooming window changing size from 100 to 1200 , using anti-symmetric mode signals, propagating from the right to the left, input frequency $13 \mathrm{kHz}$. 


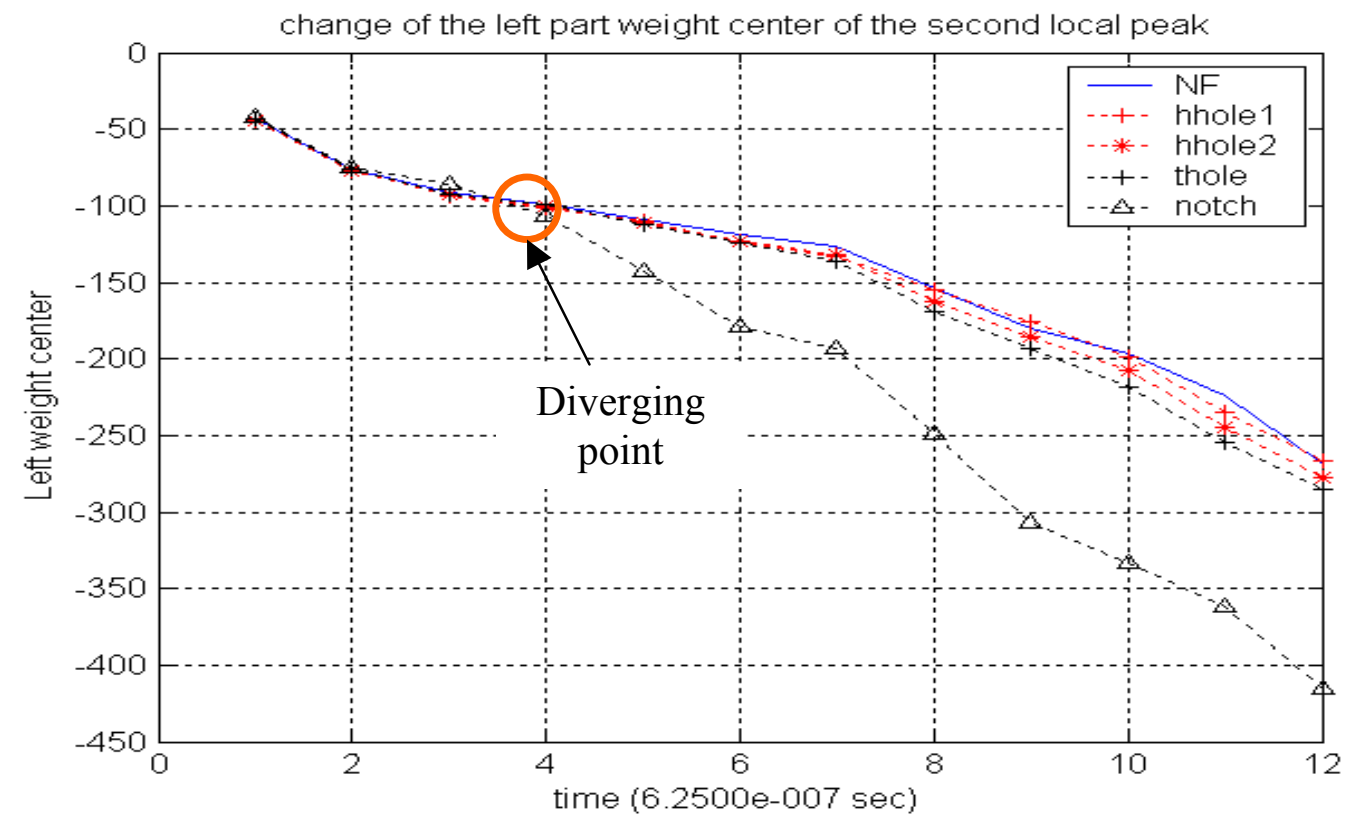

Figure 7.19. The change of left part weight center of the second local peak as the zooming window changing size from 100 to 1200, using anti-symmetric mode signals, propagating from the right to the left, input frequency $13 \mathrm{kHz}$.

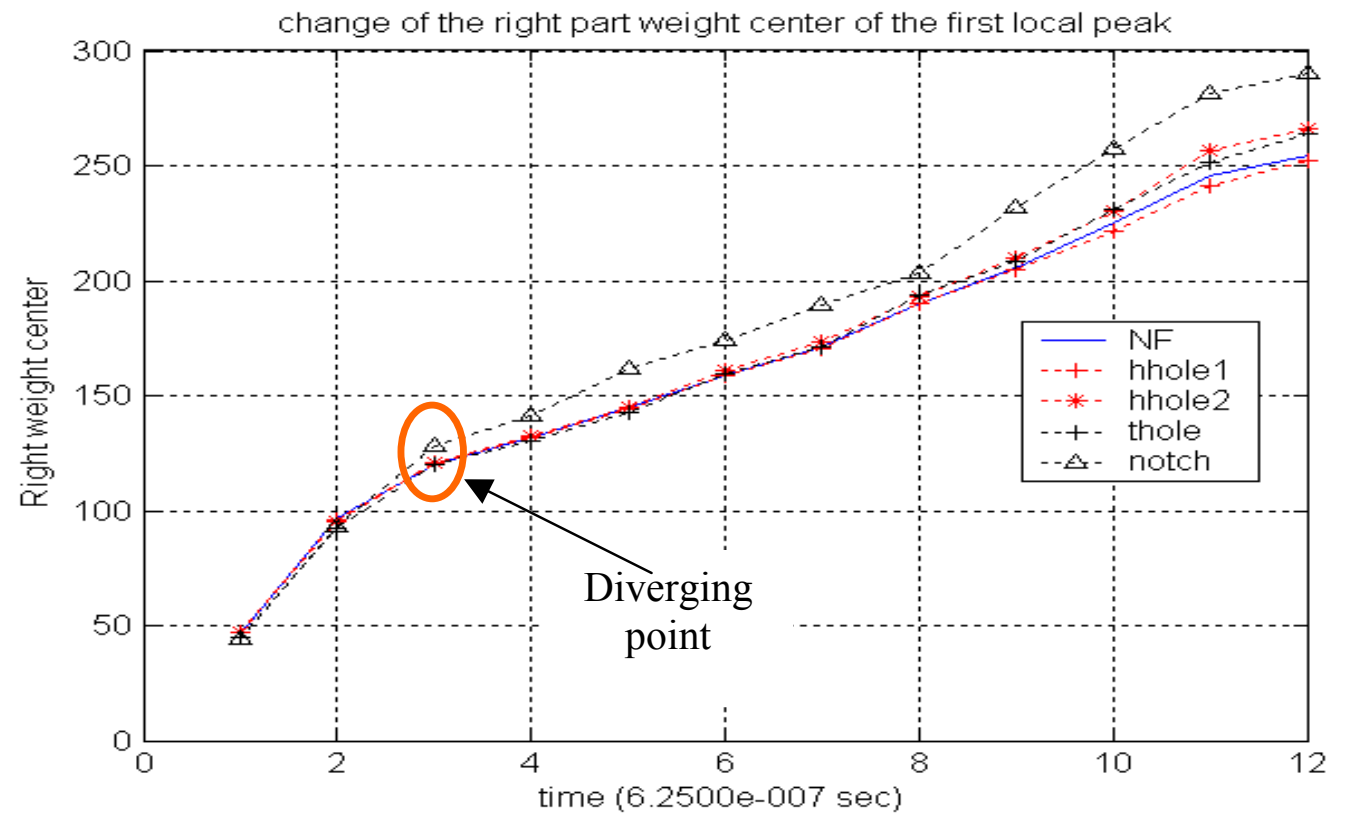

Figure 7.20. The change of right part weight center of the first local peak as the zooming window changing size from 100 to 1200 , using anti-symmetric mode signals, propagating from the left to the right, input frequency $13 \mathrm{kHz}$. 


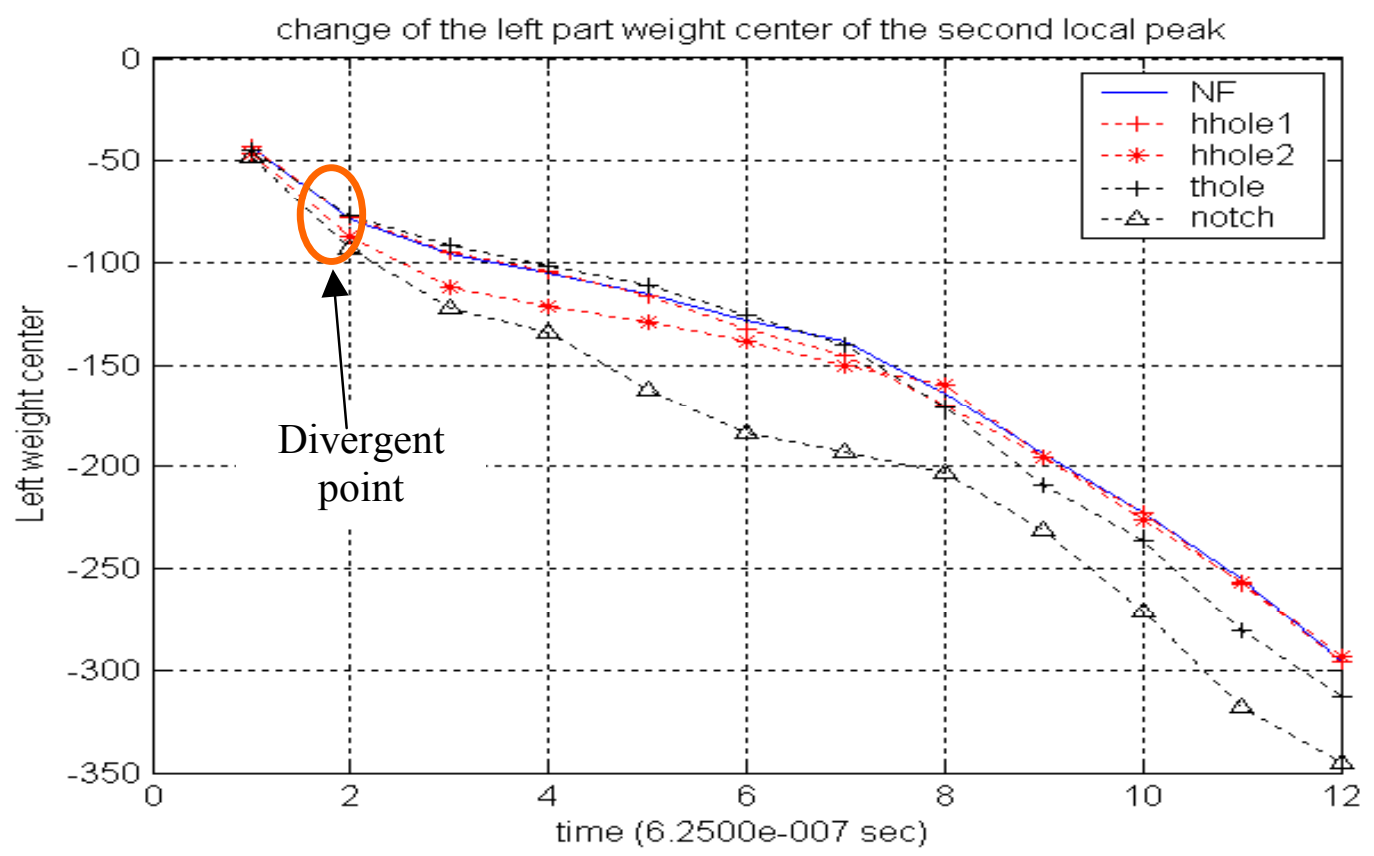

Figure 7.21. The change of left part weight center of the second local peak as the zooming window changing size from 100 to 1200 , using anti-symmetric mode signals, propagating from the left to the right, input frequency $13 \mathrm{kHz}$.

\subsubsection{Flaw Localization for Brass Tubes in Water Through Zooming Windows}

The zooming window method was tested for the tubular structure in the air. Further evaluation of this algorithm was performed and the results are presented in this section from the experiments on a brass tube in the water. The experimental conditions are listed in Table 7.2. Different flaws are created in the middle of the tube as compared with the flaws described in the previous section where the flaws are located at about onethird of the length to the left end. The results of the zooming window method are illustrated in Figures 7.22 - 7.25. It is very interesting to find that the first two local peaks have almost the same diverging point no matter whether the active signals propagate from right to left (in Figures 7.22 and 7.23) or from the left to right (Figure 7.24 and 7.25). Therefore, we are confident in predicting that the structural flaw is located in the middle of the specimen. 


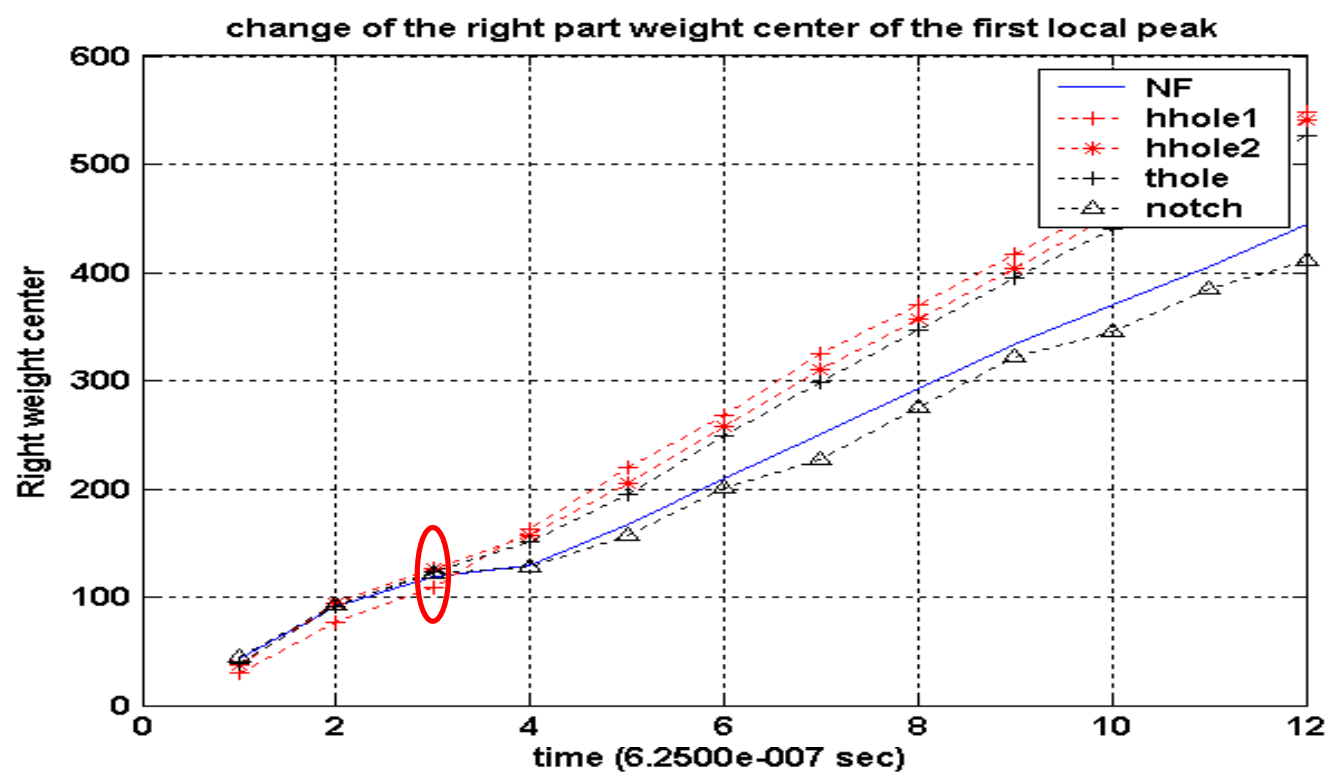

Figure 7.22. The change of right part weight center of the first local peak as the zooming window changing size from 100 to 1200 , anti-symmetric mode signals, propagating from the right to the left, input frequency $13 \mathrm{kHz}$.

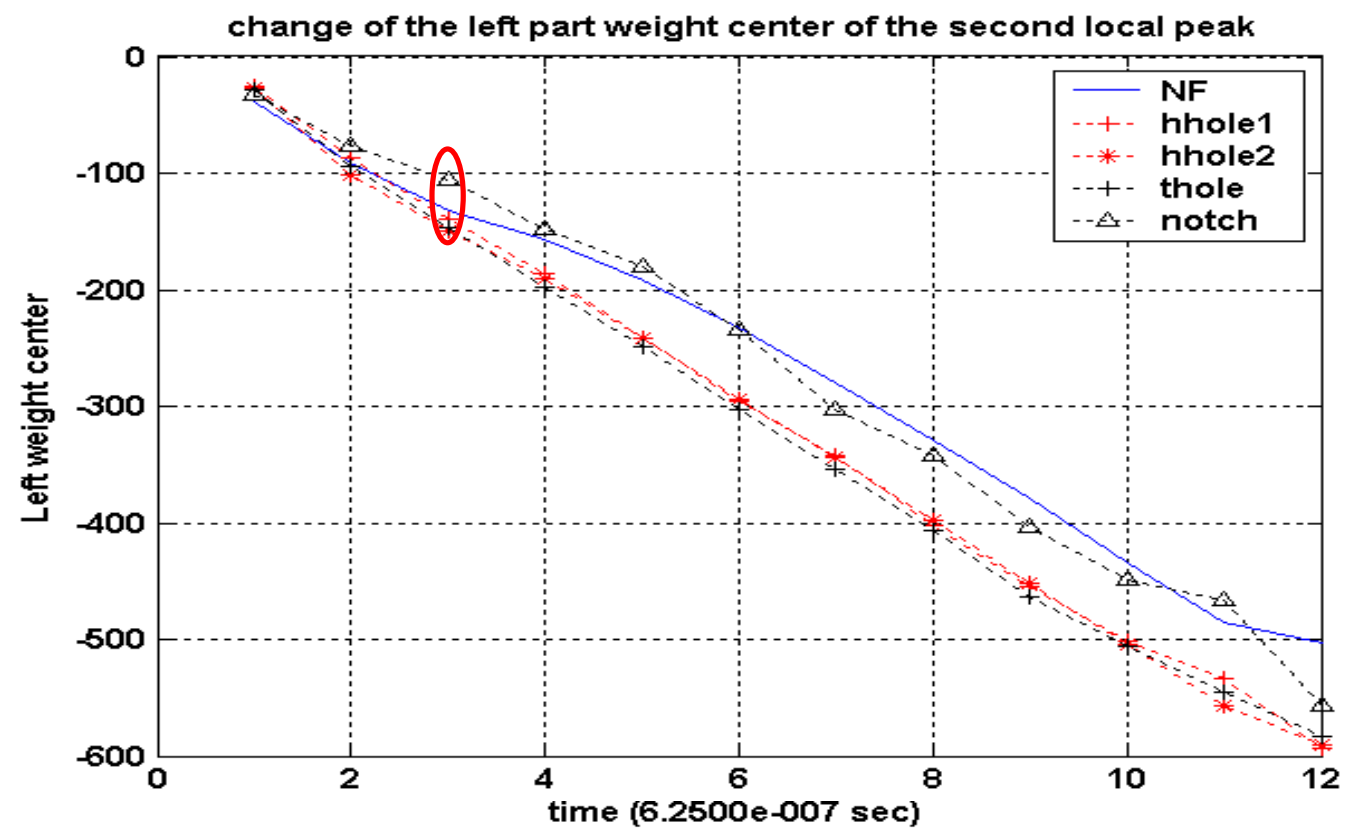

Figure 7.23. The change of right part weight center of the second local peak as the zooming window changing size from 100 to 1200 , anti-symmetric mode signals, propagating from the right to the left, input frequency $13 \mathrm{kHz}$. 


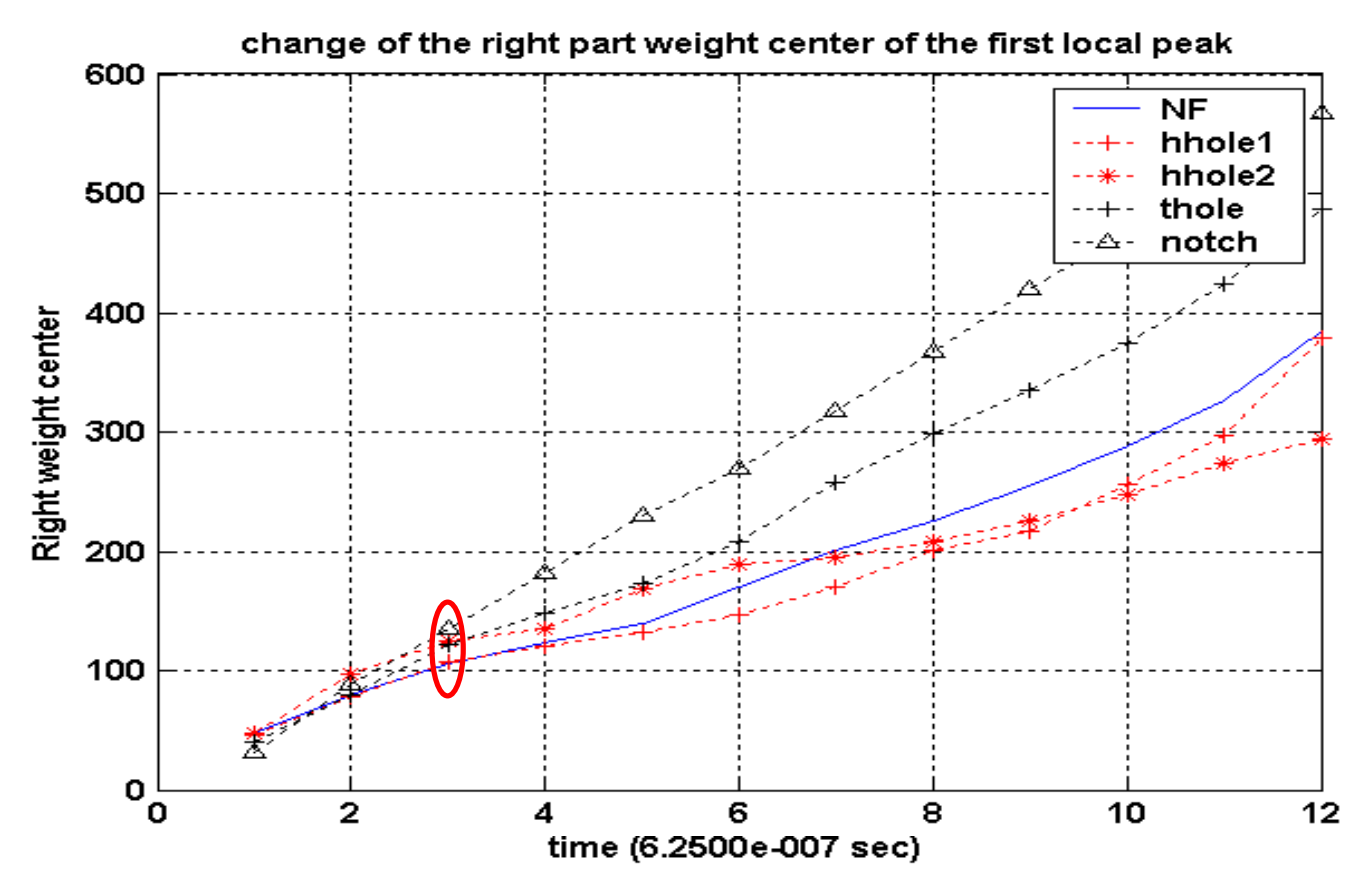

Figure 7.24. The change of right part weight center of the first local peak as the zooming window changing size from 100 to 1200 , anti-symmetric mode signals, propagating from the left to the right, input frequency $13 \mathrm{kHz}$.

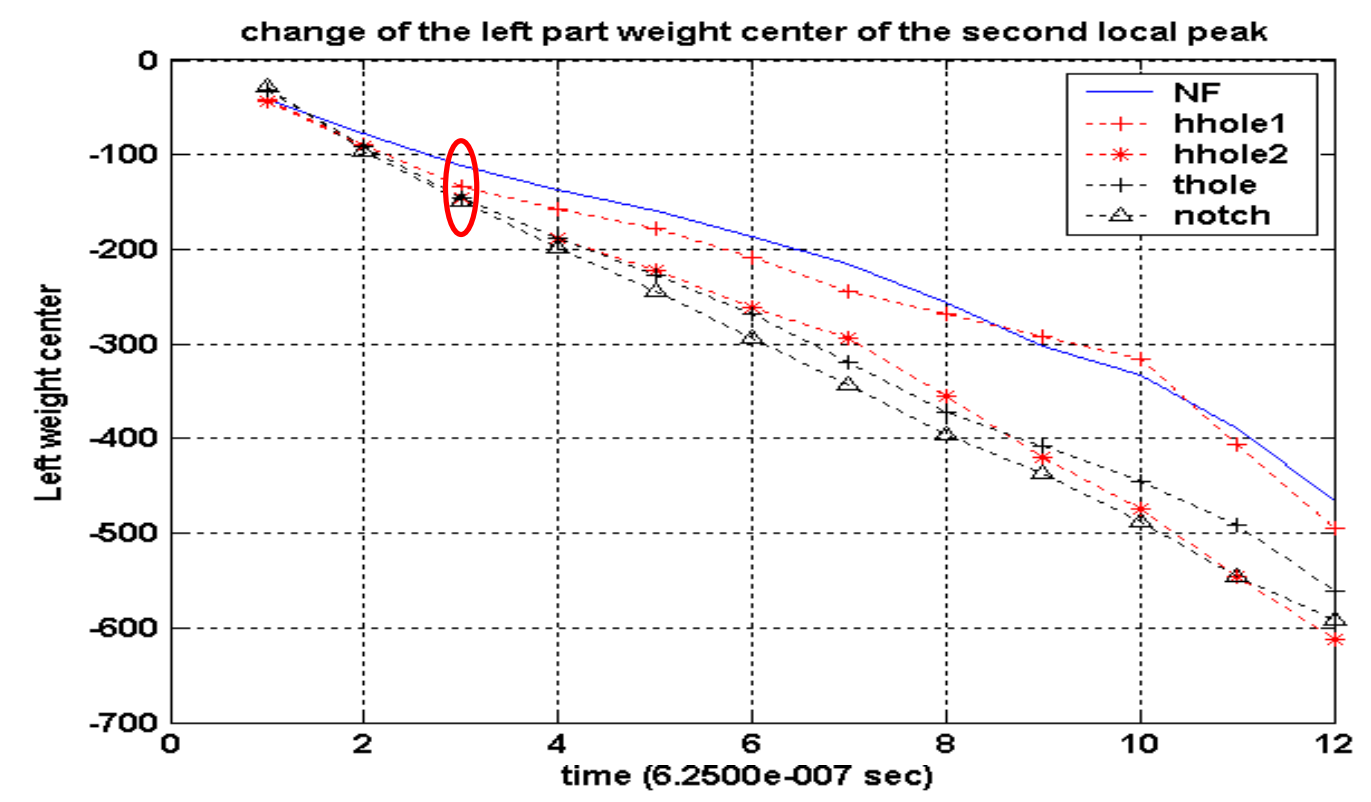

Figure 7.25. The change of right part weight center of the second local peak as the zooming window changing size from 100 to 1200 , anti-symmetric mode signals, propagating from the left to the right, input frequency $13 \mathrm{kHz}$. 


\subsection{Noise Reduction of Acoustic Signals in Brass Tubes}

Under a noisy environment, the structural monitoring proposed in this research becomes complicated. Because the noise may cause the signal processing techniques mentioned above invalid. Hence a de-noising process is necessary.

There are two types of noise in a steam generator system, the high frequency noise caused by steam bubbles and structural vibrations, and the low frequency noise caused by the turbulence of water flow. The noisy environment was simulated in the experiments during this research. The noisy raw signals can be observed in the following figures. We used HHT as an adaptive de-noising algorithm to extract useful signals in this project. We first decomposed the raw signals into multiple IMFs as shown in Figures 7.22 and 7.23. The number of levels was adjustable based on the complexity of the environment. Then a purified time series was reconstructed by summarizing selected useful IMFs as shown in Figure 7.24.

The results illustrate that the representative signals can be perfectly extracted from noisy data, and the purified data are suitable for the implementation of the proposed signal processing techniques.

Figures $7.25-7.28$ illustrate the features extracted through the moving window method after HHT de-noising process. Note that the features tend to become uniform at the end of the data. This is because the acoustic energy decreases quickly in the water such that the local peaks are not so obvious after the first several peaks. Therefore, focus should be put on the first four to five local peaks depending on the input signal amplitude and the properties of water. Very good performance was achieved using moving window after HHT de-noising. While the features from same process before de-noising were not quite meaningful as shown in Figure 7.29, where the difference of the spread of the local peaks under different conditions should increase with time. In contrast Figure 7.26 gives the correct answer after noise reduction. Similar phenomenon occurred for the local peak gravity center. The left and right gravity center of the local peaks could not generate meaningful features under the effect of the two-phase flow noise in water. This is illustrated in Figures 7.30 and 7.31. 
As discussed in Section 7.2, the local peak amplitude is sensitive to the change of boundary conditions, therefore we found the abnormal change of amplitude under notch condition as shown in Figure 7.25. However, other features such as the gravity center position are not significantly affected by the boundary conditions.

In summary, the HHT noise reduction method worked very well for the brass tubes under the effect of two-phase flow environment. The recovered signals generated good features for tubular specimens using the moving window technique.

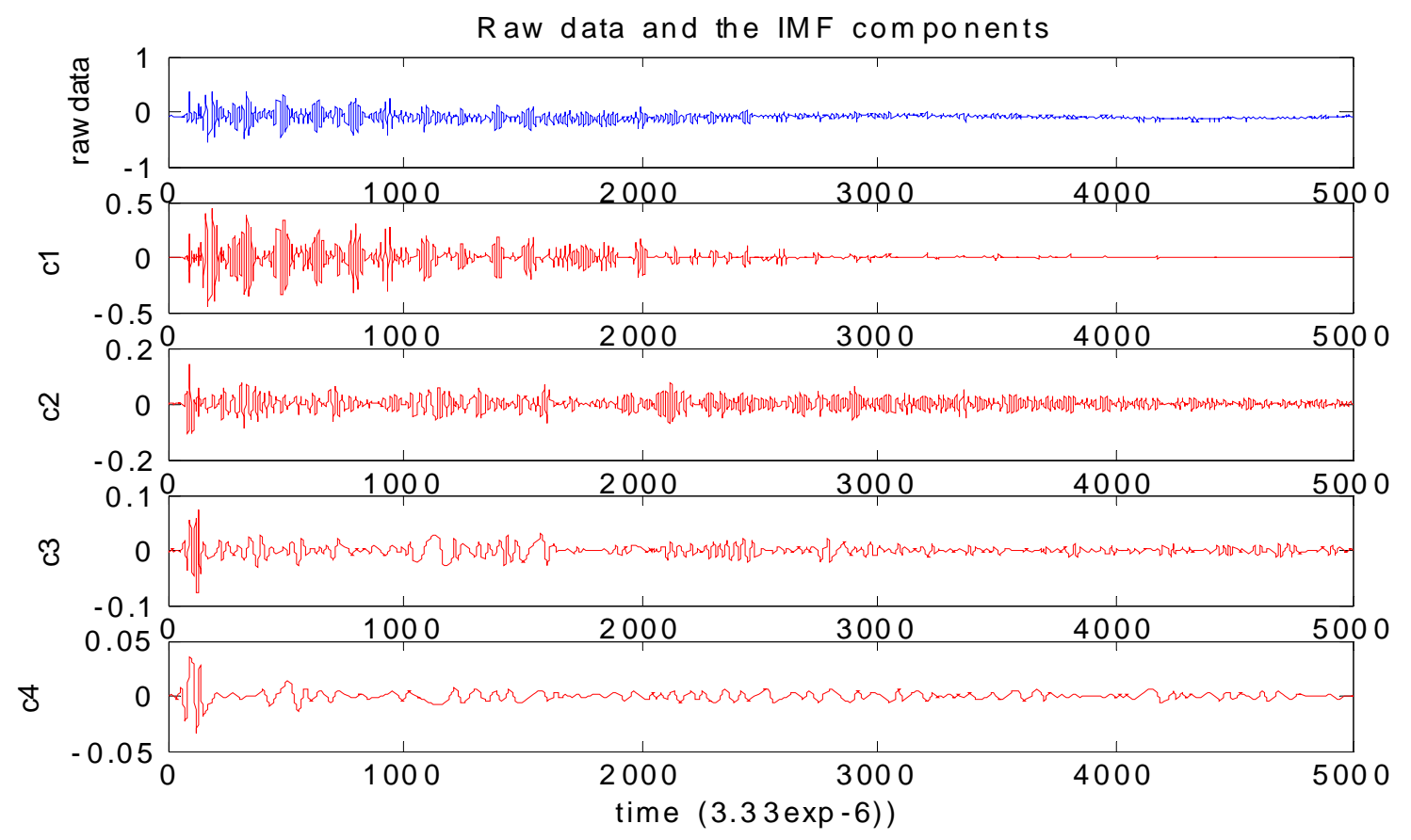

Figure 7.22. Raw data and IMFs from HHT. 


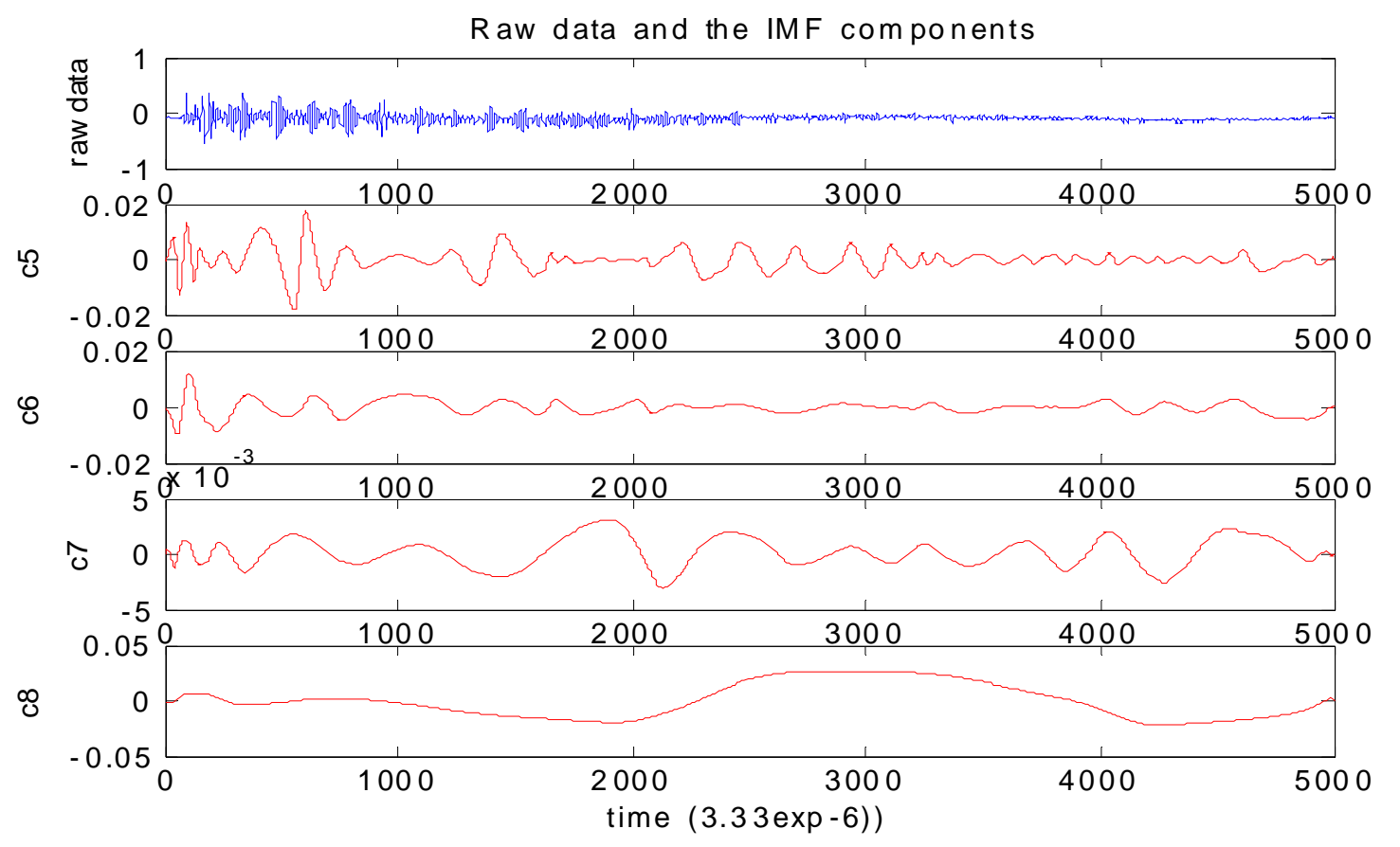

Figure 7.23. Raw data and IMFs from HHT.

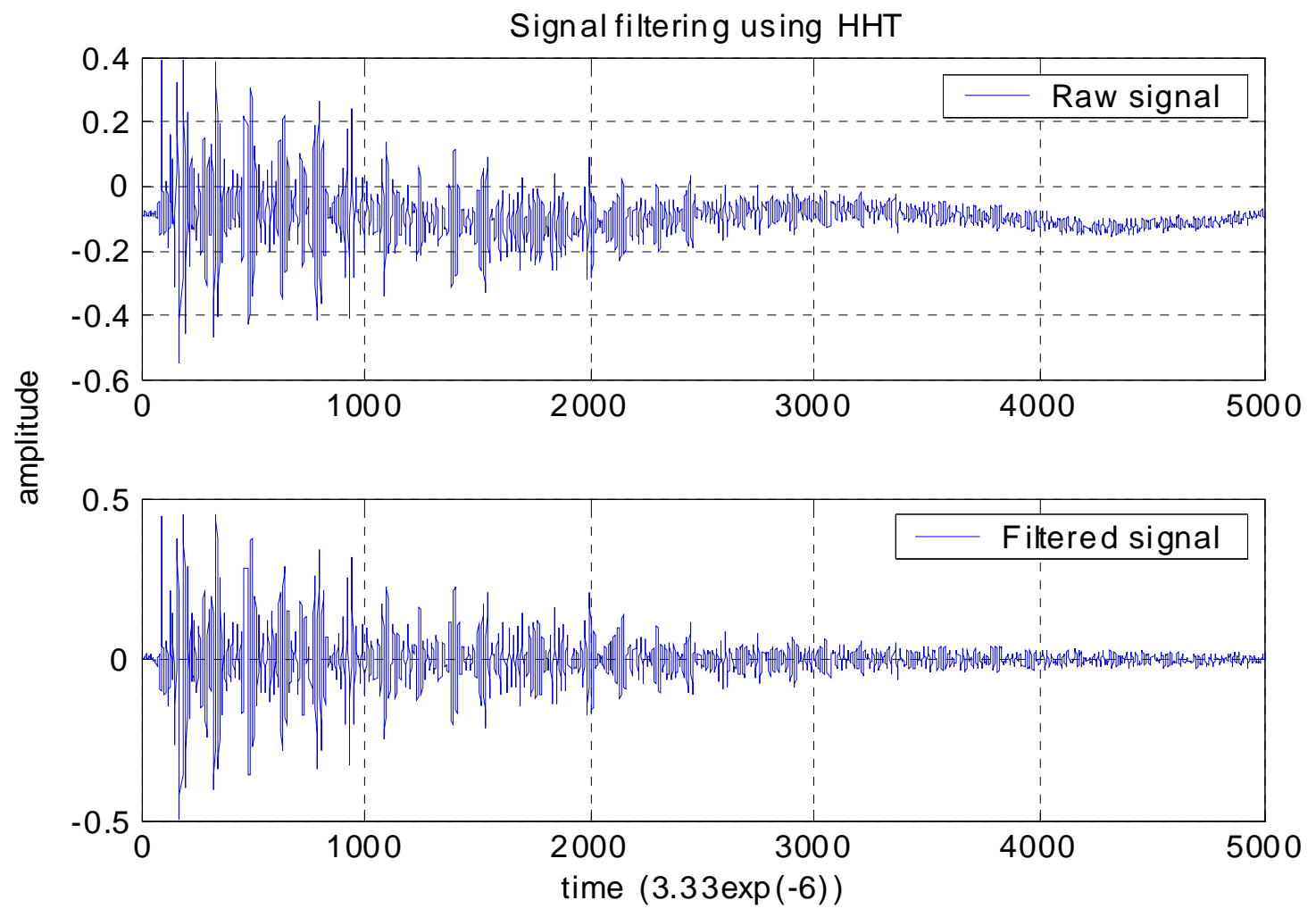

Figure 7.24. Raw data and the reconstructed data. 


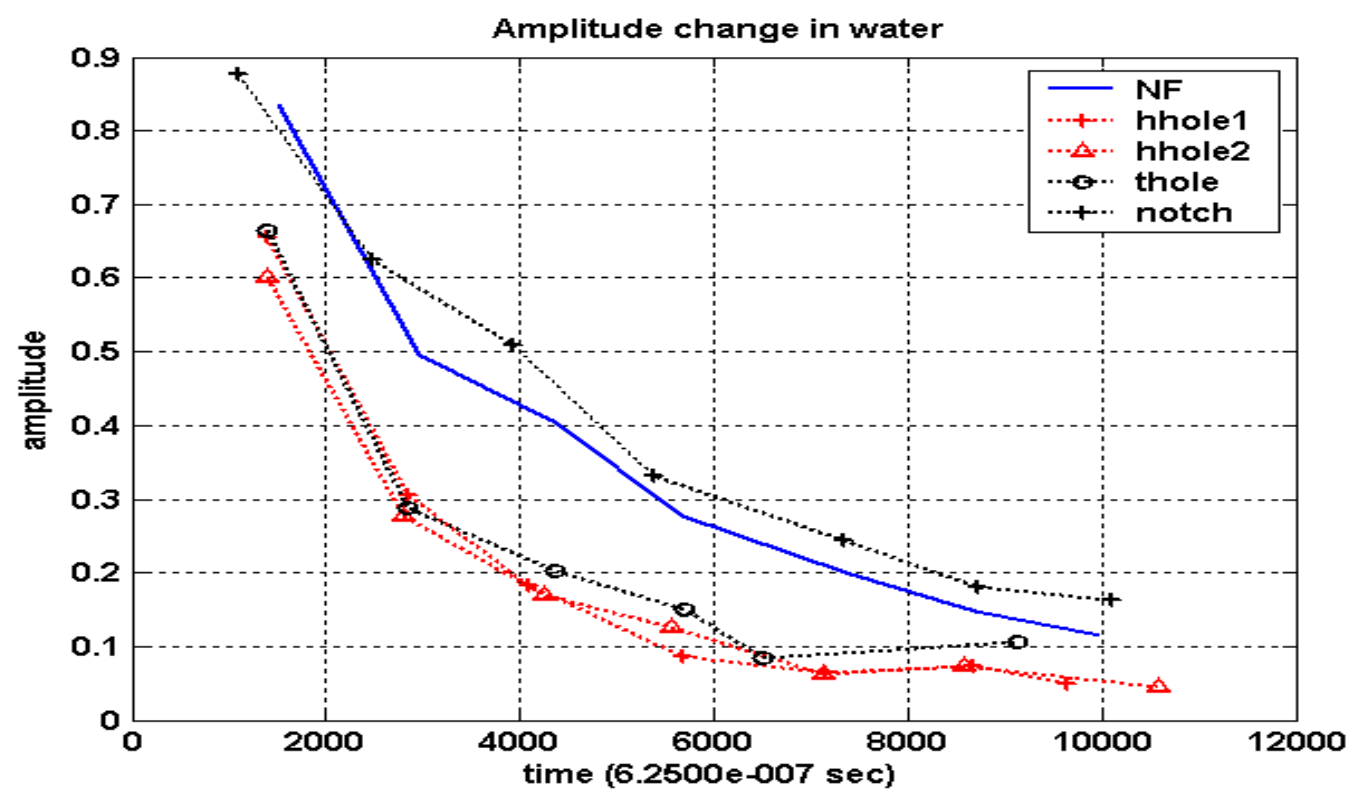

Figure 7.25. Amplitude change of local peaks from anti-symmetric mode signal after denoising, with input frequency $13 \mathrm{kHz}$.

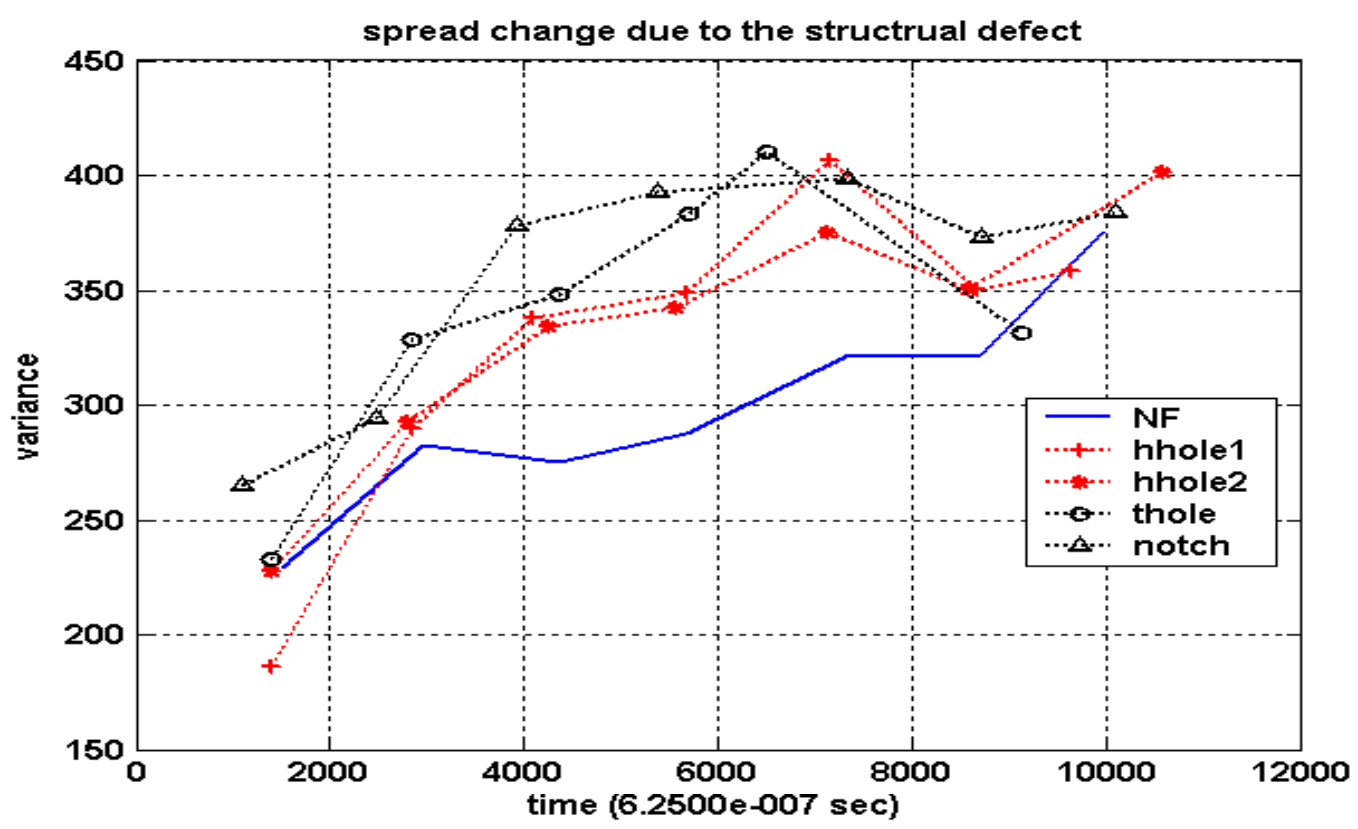

Figure 7.26. Spread change of local peaks from anti-symmetric mode signal after denoising, with input frequency $13 \mathrm{kHz}$. 


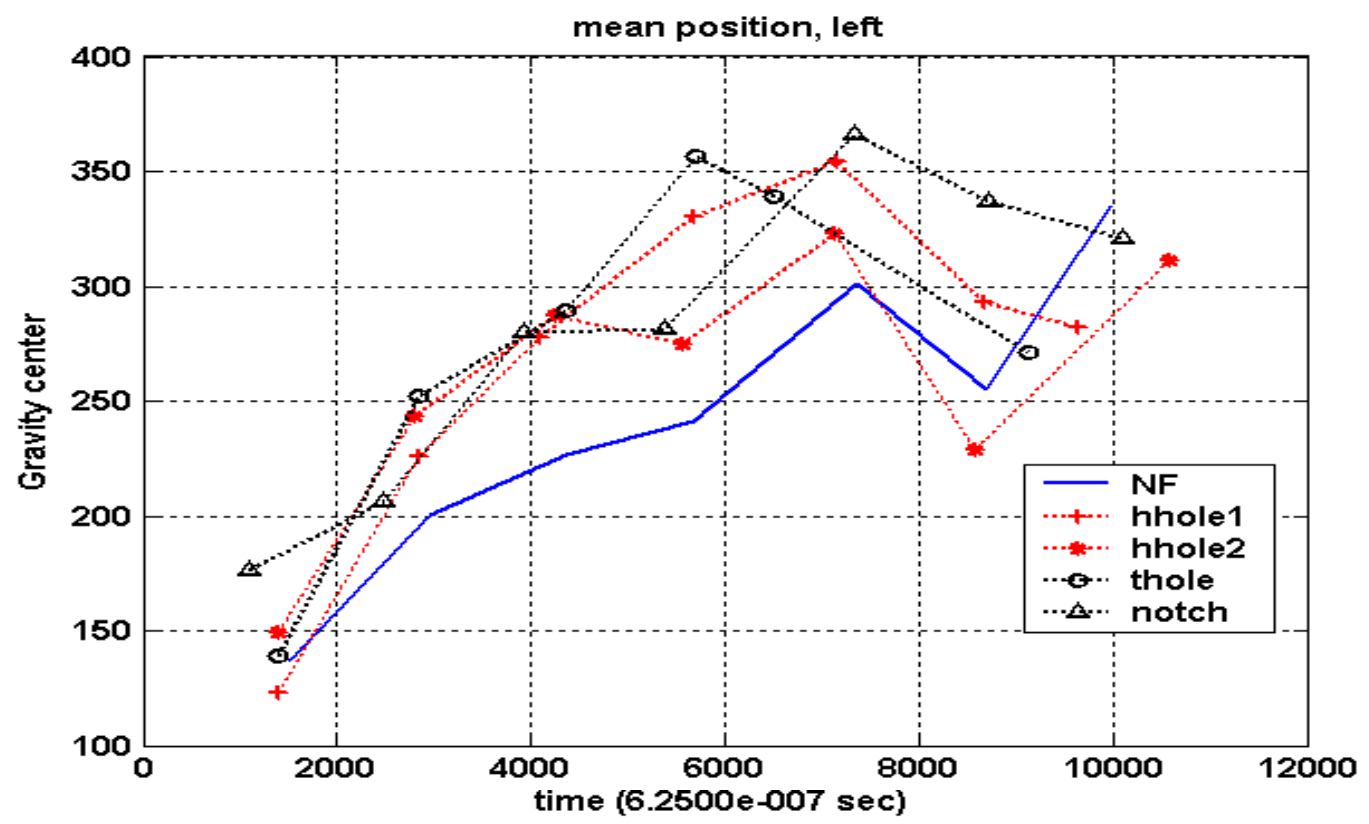

Figure 7.27. Left gravity centers of local peaks from anti-symmetric mode signal after de-noising, with input frequency $13 \mathrm{kHz}$.

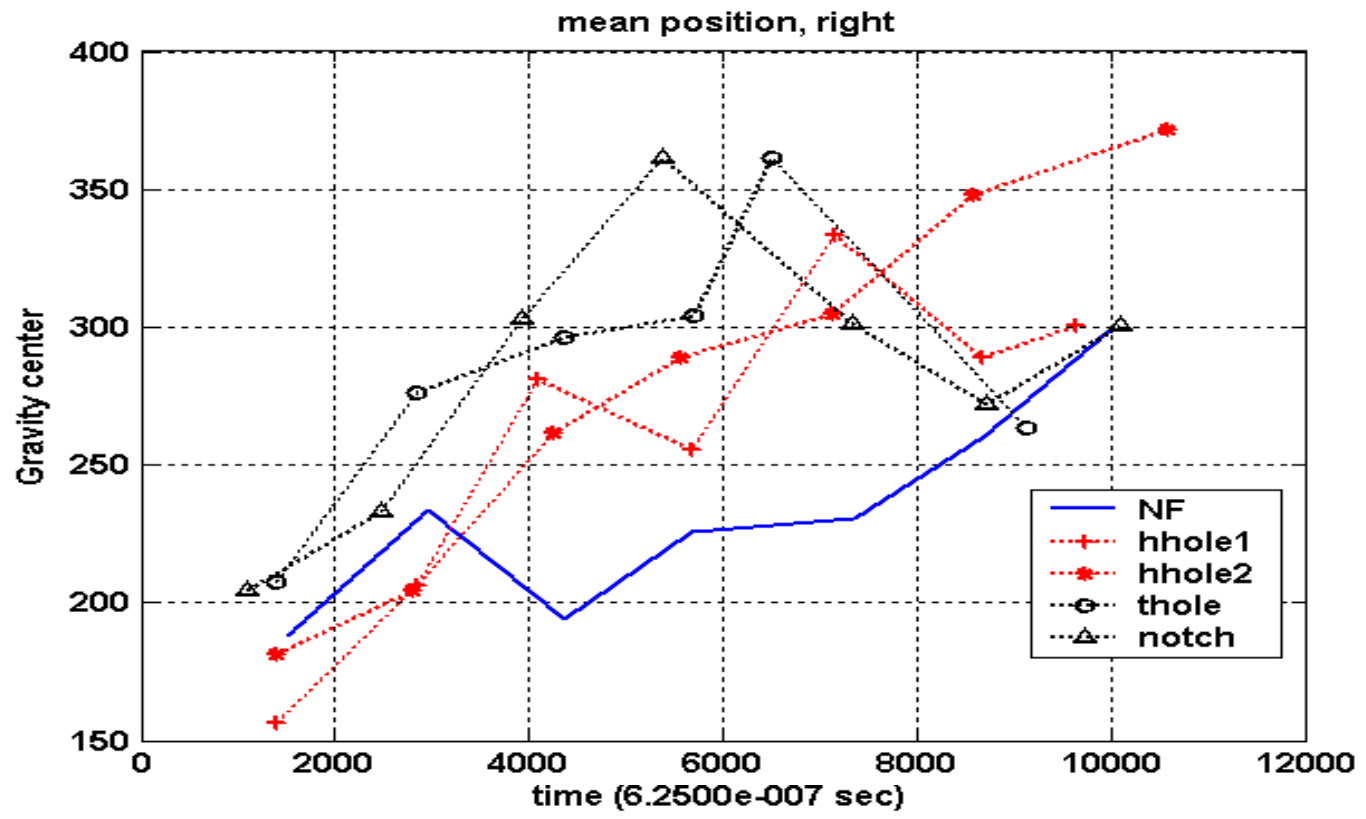

Figure 7.28. Right gravity centers of local peaks from anti-symmetric mode signal after de-noising, with input frequency $13 \mathrm{kHz}$. 


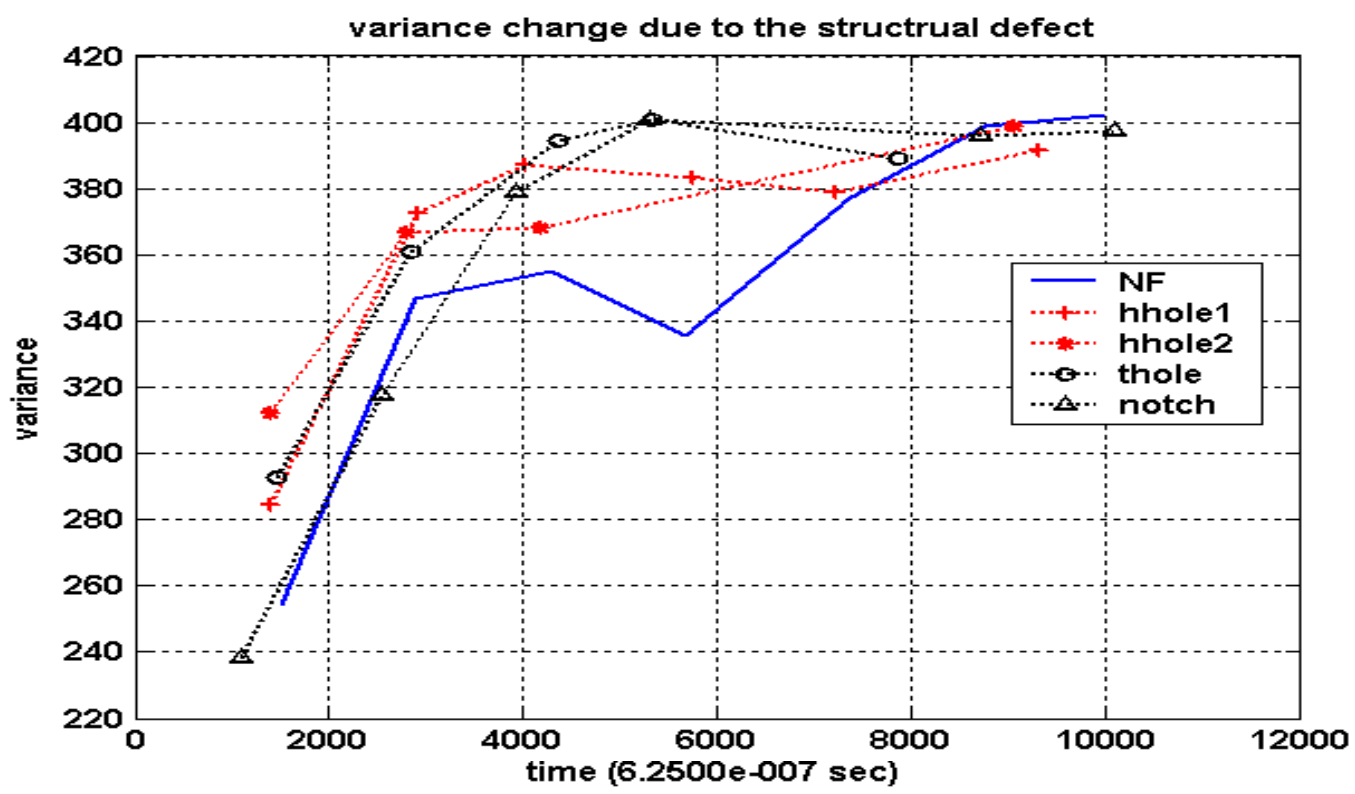

Figure 7.29. Spread change of local peaks from anti-symmetric mode signal before denoising, with input frequency $13 \mathrm{kHz}$.

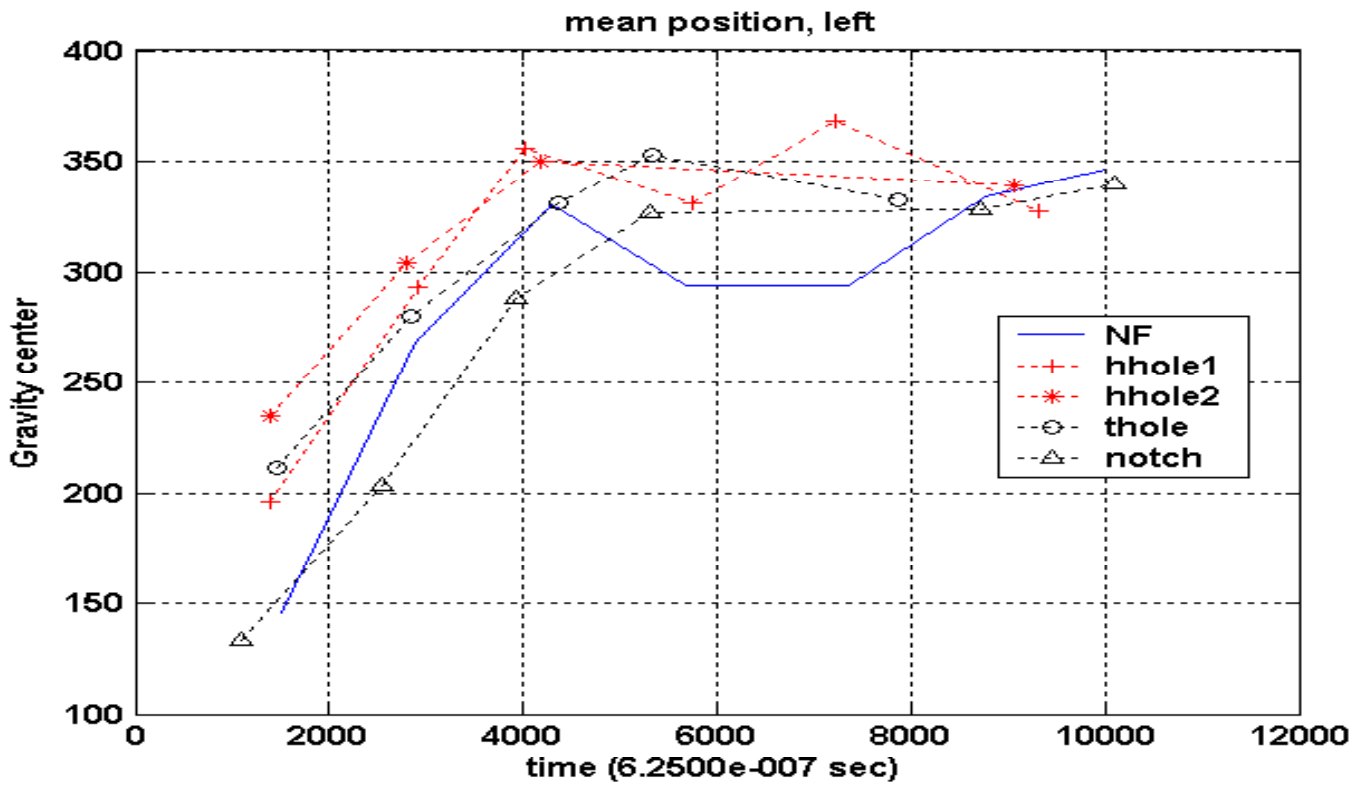

Figure 7.30. Left gravity centers of local peaks from anti-symmetric mode signal with noise, with input frequency $13 \mathrm{kHz}$. 


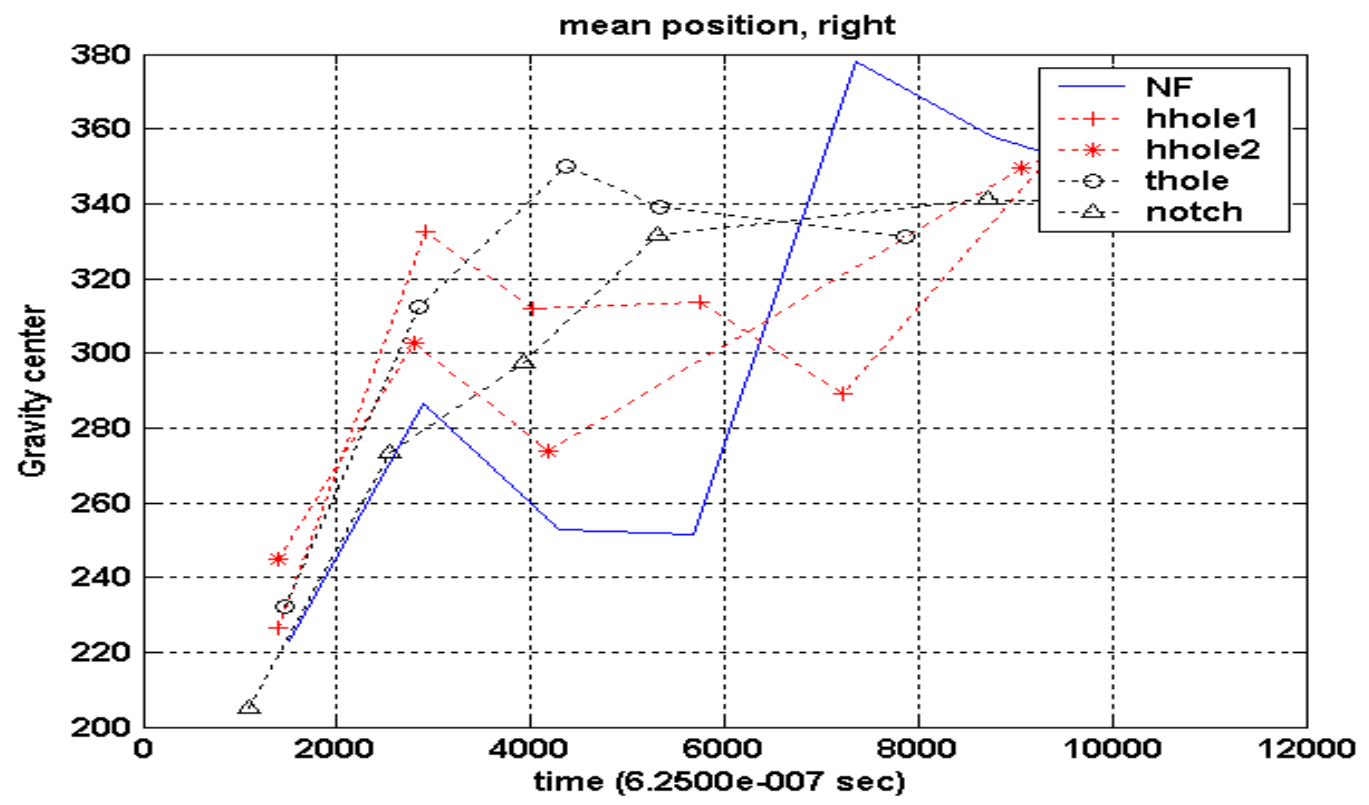

Figure 7.31. Right gravity centers of local peaks from anti-symmetric mode signal with noise, with input frequency $13 \mathrm{kHz}$.

\subsection{Classification of Tube Flaws}

The accuracy of the classification for defects in tubular structures depends primarily on the selection and extraction of representative features. In this research, the features extracted through moving window technique are used for defect classification. Five structural conditions were trained and the representative feature matrices, defined in previous sections were collected for classification. Experimental data were collected for each structural condition under different input frequency, and the results generated by the moving window method were used for classification. The normalized distance between the tested matrix and the trained matrices were calculated and listed in Table 7.4. We can see the matrices for partial hole and through hole defects have similar distance to a test matrix, so it is easy to misclassify them. The reason is that these three tube flaws, in fact, have similar features. Therefore other classification methods and representative features were also explored to increase the success rate of the method implemented here These are discussed later. 
Table 7.4. Distance between test and training matrices

\begin{tabular}{|l|l|l|l|l|l|}
\hline Test & Nraining & $\begin{array}{l}\text { Half hole } \\
(0.6 \mathrm{~mm})\end{array}$ & $\begin{array}{l}\text { Half hole } \\
(1.8 \mathrm{~mm})\end{array}$ & $\begin{array}{l}\text { Through } \\
\text { hole }(0.6 \mathrm{~mm})\end{array}$ & Notch \\
\hline NF & 0.0272 & 0.0568 & 0.0597 & 0.0650 & 0.4048 \\
\hline Half hole $(0.6 \mathrm{~mm})$ & 0.0224 & 0.0074 & 0.0058 & 0.0105 & 0.2745 \\
\hline Half hole $(1.8 \mathrm{~mm})$ & 0.0418 & 0.0216 & 0.0087 & 0.0064 & 0.2010 \\
\hline Through hole $(0.6 \mathrm{~mm})$ & 0.0573 & 0.0311 & 0.0144 & 0.0082 & 0.1765 \\
\hline Notch & 0.4564 & 0.3395 & 0.2760 & 0.2406 & 0.0118 \\
\hline
\end{tabular}

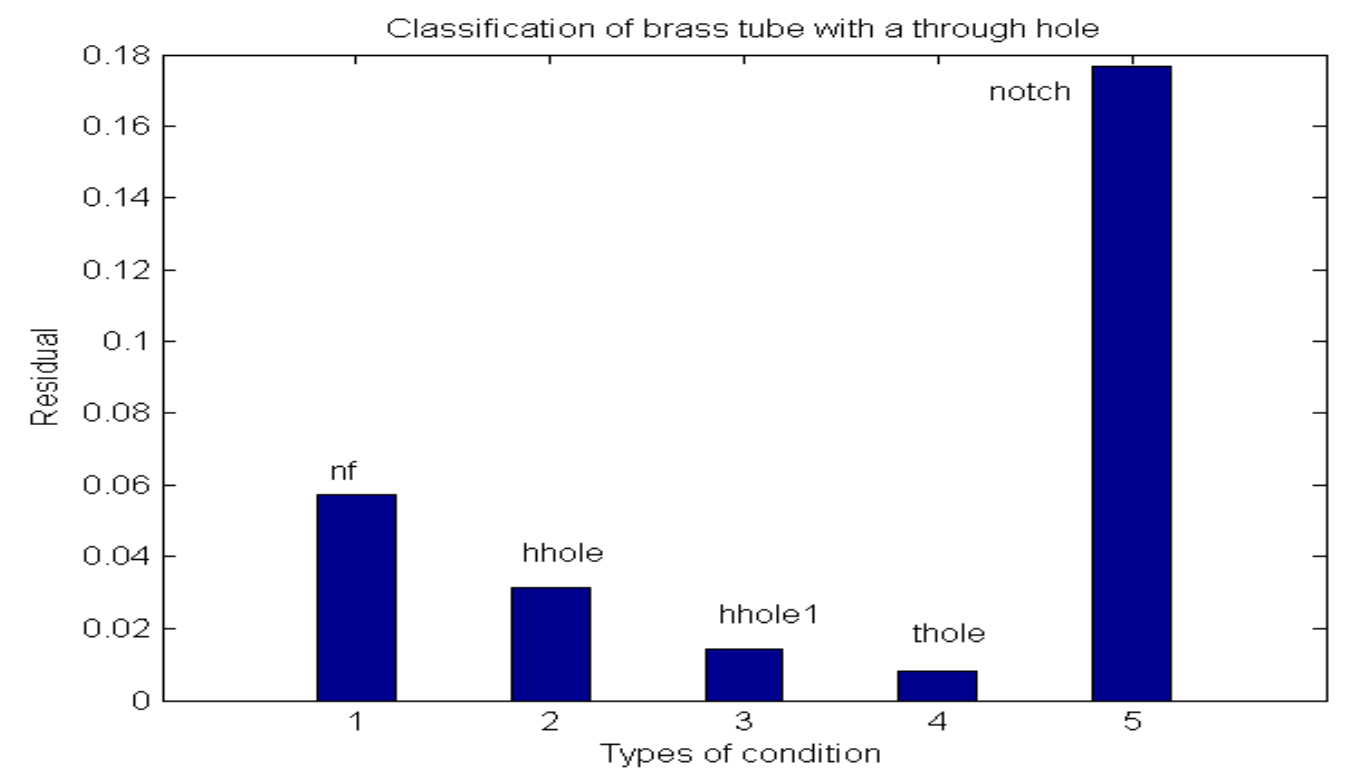

Figure 7.32. The classification of tube defects. 
Figure 7.32 illustrates the plots of the measurement residuals between the test and the training conditions. Based on this plot, the test defect was classified into a through hole in a tube, which is the actual condition in our study.

In this research another technique, DWT + eigen face analysis, was tested for the feature extraction and classification of tube flaws. The method used DWT and Eigenface analysis. The efficiency of DWT makes the process of classification much faster than continuous wavelet analysis. In the example illustrated in Section 7.3, the raw signals with active frequency of $16 \mathrm{kHz}, 17 \mathrm{kHz}, 18 \mathrm{kHz}$, and $19 \mathrm{kHz}$ were decomposed into five levels according to the properties of the input. The decomposed signals of acoustic waves are plotted in Figure 7.33. An Eigen-face was extracted to represent a structural condition. The test signals were then projected onto the space spanned by an Eigen-face, a residual between the test $\mathrm{T}-\mathrm{F}$ and the recovered one using principal components and corresponding scores was generated. The test condition was classified into the flaw type that has the smallest residual. As shown in Figures 7.34 and 7.35, six types of structural conditions were trained and tested using Lamb wave signals from brass tubes, and all of the conditions were correctly classified. The six conditions of brass tubes were: normal condition in air, a half hole on a tube in the air, a through hole on a tube in the air, normal condition in water, a haft hole on a tube loaded with water, and a through hole on a tube loaded with water. 


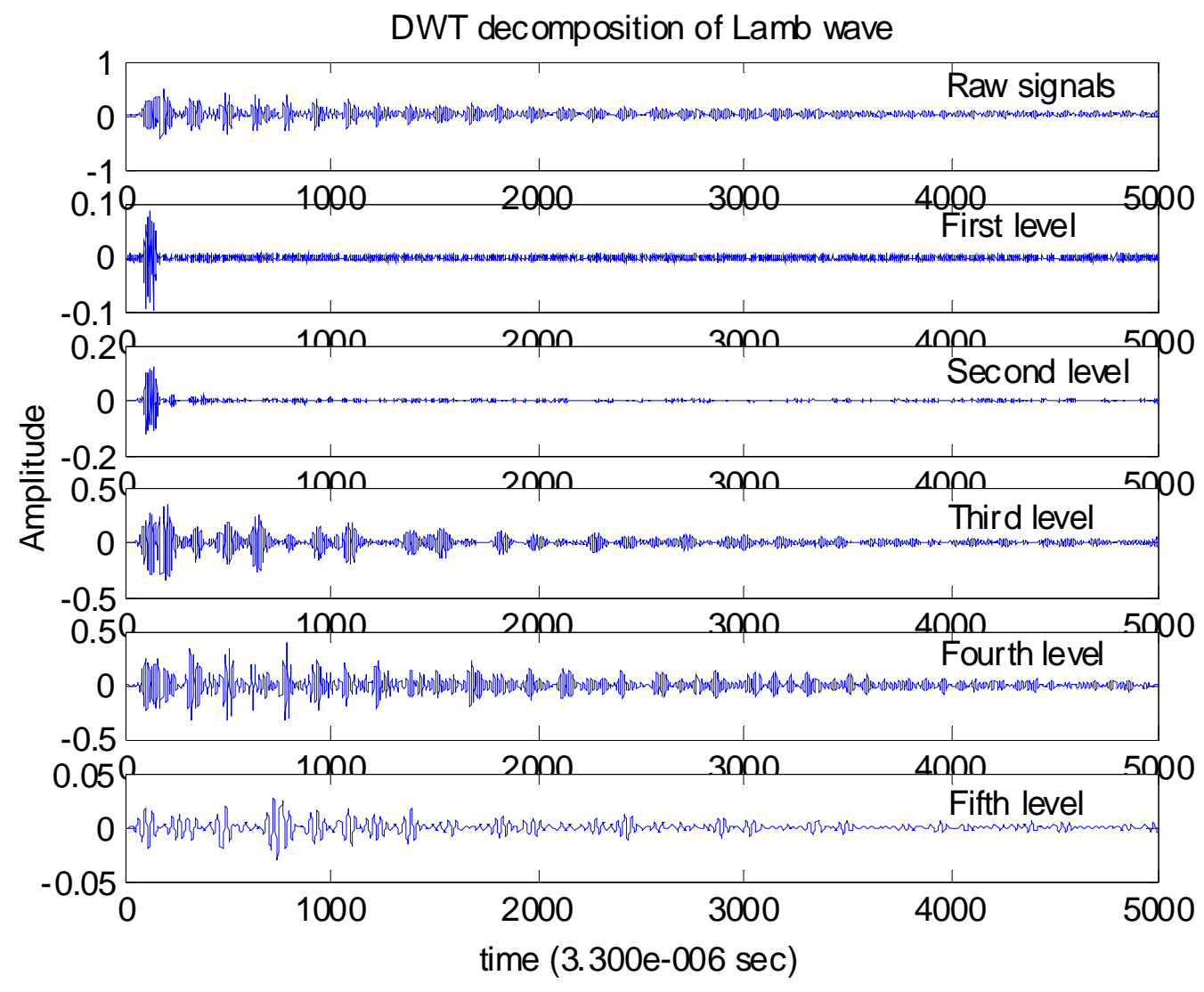

Figure 7.33. DWT decomposition of acoustic waves in a brass tube. 


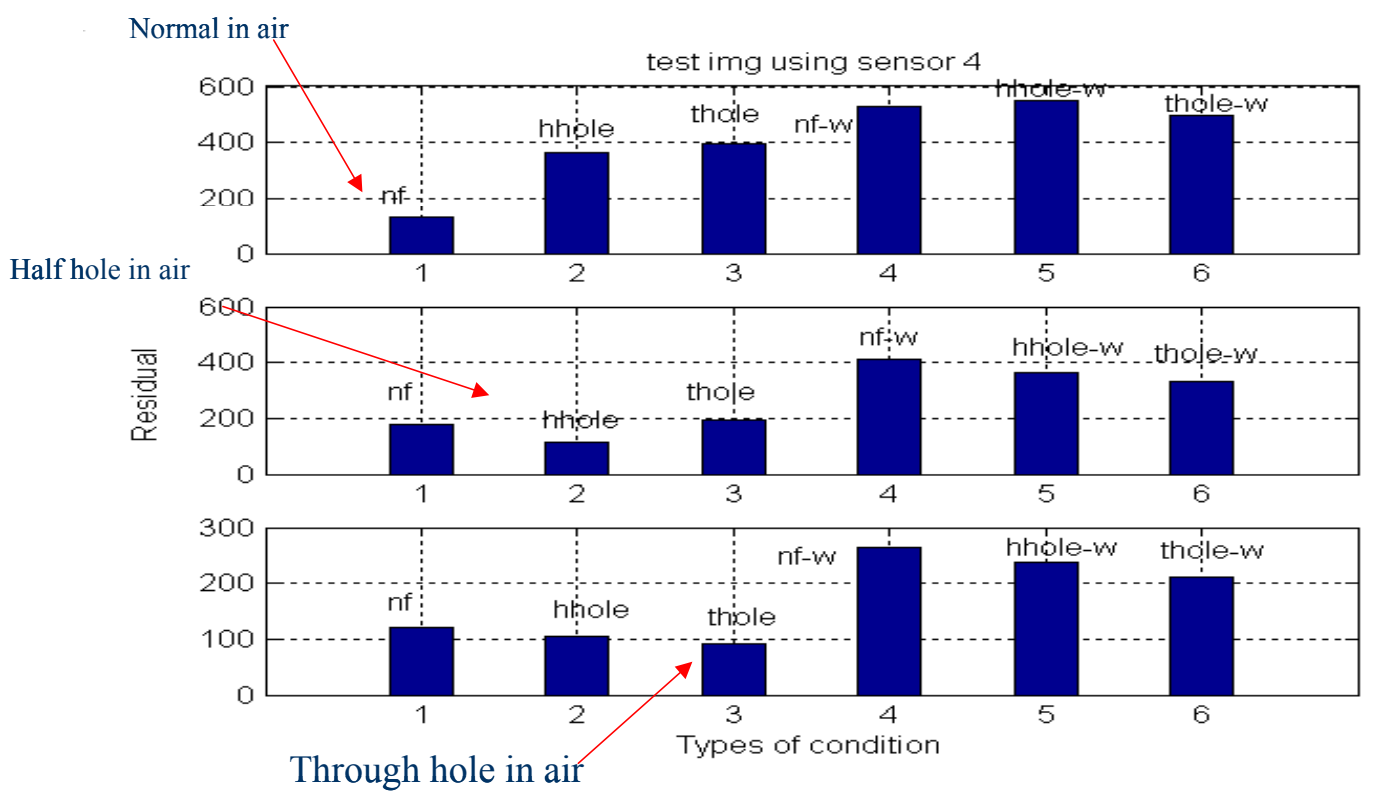

Figure 7.34. The tube defect classification using DWT + Eigen-face.

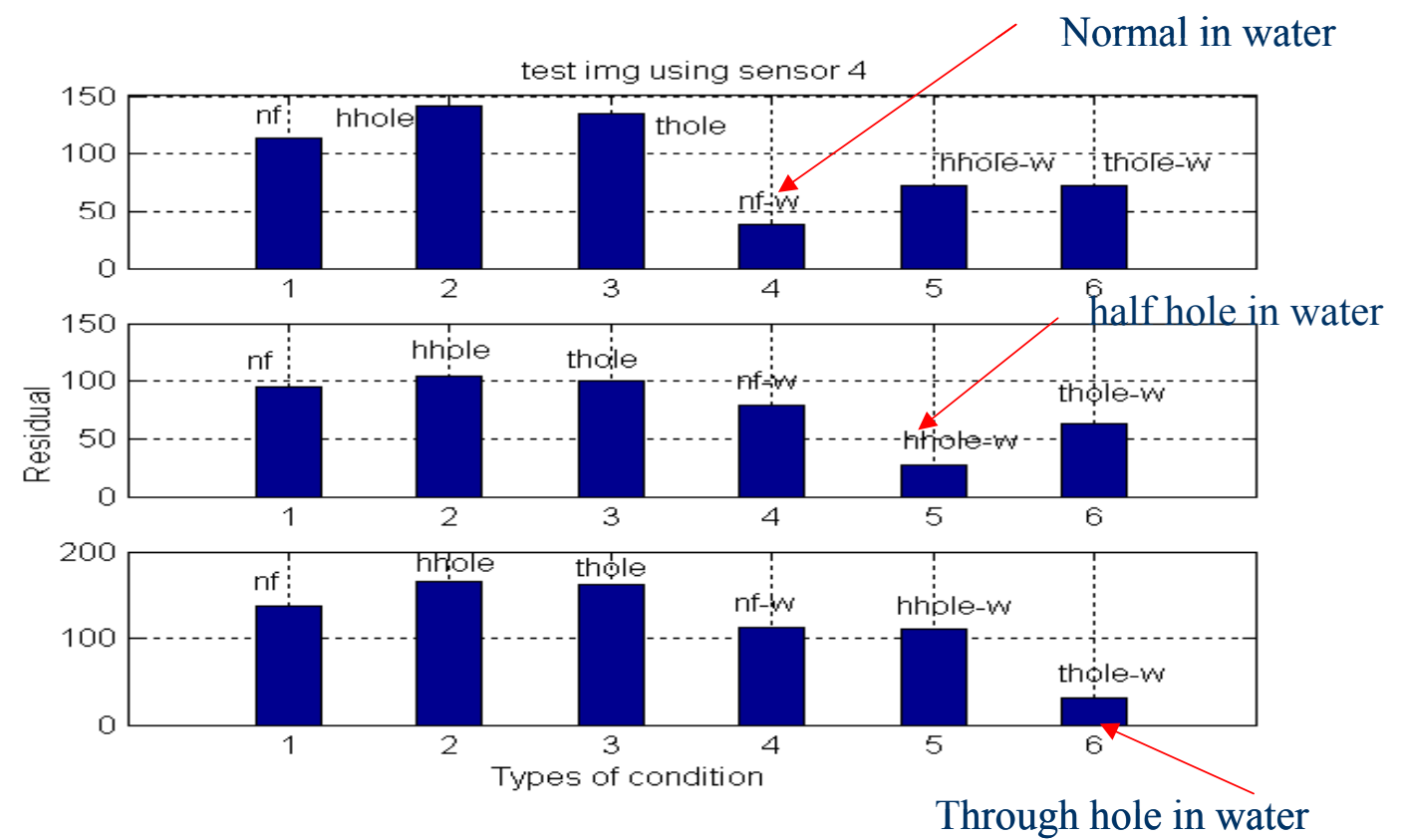

Figure 7.35. The tube defect classification using DWT + Eigen-face. 


\subsection{Summary of Tubular Structural Evaluation}

The tubular structures were evaluated through Lamb wave analysis. The structural evaluations were performed both in water and in air. An obvious bias caused by water pressure is found in the experiments under water shown in Figure 7.36. The bias must be eliminated before acoustic signal processing.

The moving-window technique was applied in this chapter for the feature extraction in tubular structure. The results of analysis demonstrate the effectiveness of this method in both water and in air. It can be used to both detect the structural flaws and evaluate the severity. The analysis also shows that the anti-symmetric Lamb waves are more sensitive to the structural changes compared with symmetric signals. An optimal window size is a key parameter for moving window method to be successful.

The zooming window technique was used to estimate the flaw location if an anomaly was detected. The method worked well in generating an approximate flaw position in the air and water without the effect of two-phase flow. This technique was not used to calculate an accurate flaw position, but it is applicable to many cases.

The noisy environment was simulated through the two-phase flow. The moving window method was not able to generate meaningful result under the effect of noise. Therefore the HHT technique was introduced for the purpose of de-nosing. The moving window method generates satisfactory results using de-noised acoustic signals.

Two types of classification methods were tested in this chapter for tubular flaws. The first one used the features from moving windows and calculated the distance among the tubular conditions as listed in Table 10.4. We can see that some similar flaws like the half hole 1 , haft hole 2 , and the through hole are not easy to separate using this method. Hence another method using WT + Eigen-face was implemented. This method had generated better classification results. All the tested flaws (in air and in water) were identified. One shortcoming of this method is that it seeks to use all the redundant information in raw signals, thus it takes more calculation time and may not be robust when checking a tubular specimen using features from another tube. 
In summary, this section provides the advanced signal processing techniques for the tube like structure flaw detection, severity evaluation, flaw localization, noise reduction, and classification.

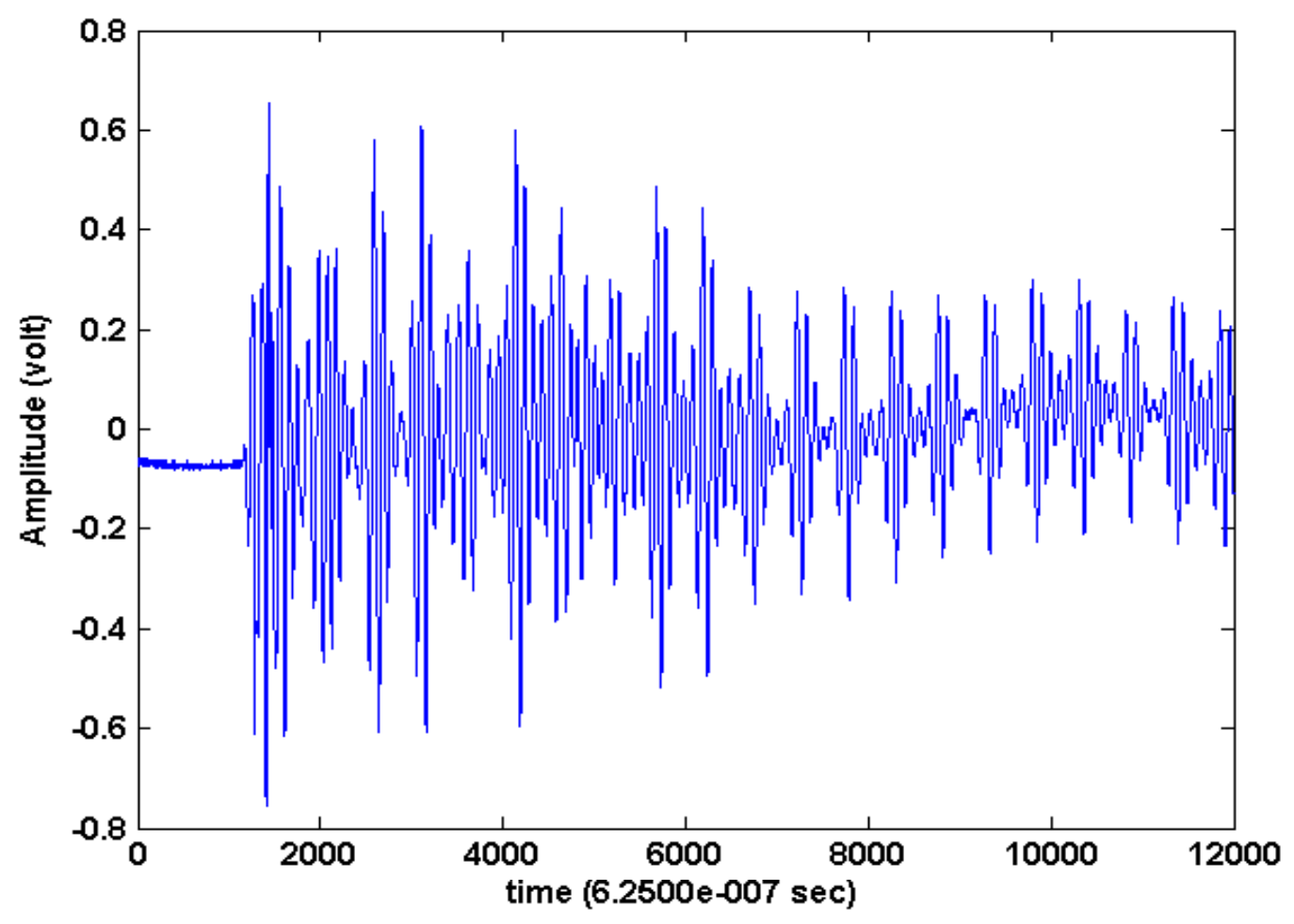

Figure 7.36. Lamb wave signals with noise from a brass tube. 


\section{SIMULATION OF LAMB WAVE PROPAGATION USING THE FINITE ELEMENT CODE ABAQUS}

\subsection{Introduction}

The simulation of Lamb wave propagation along plate and tube like structures was an important task to verify our experimental and theoretical results. Several finite element codes such as FEMLAB, ENSYS, and ABAQUS were tested. Among them, $A B A Q U S$ was selected for its good capability in simulating acoustic wave propagation in solid media. In general, ABAQUS is a highly sophisticated software package developed by Hibbitt, Karlsson \& Sorensen, Inc. [95] for modeling the behavior of solids and structures under externally applied loading. ABAQUS includes the following capabilities:

a) Simulation of both static and dynamic stress distribution problems.

b) Dynamic studies of linear and nonlinear systems.

c) Modeling large shape changes in solids, in both two and three dimensions.

d) Heat transfer.

e) Modeling the contact between solids.

f) Eigenvalue buckling prediction.

g) Natural frequency extraction.

h) Models for foams, concrete, soils, piezoelectric materials, and many others, based on an advanced material library.

i) Modeling a number of interesting phenomena, including vibration, coupled fluid/structure interactions, acoustics, buckling problems, and others.

$A B A Q U S$ is used by a wide range of industries, including aircraft manufacturers, automotive industry, oil companies, microelectronics industries, as well as national laboratories and universities. The flow chart for ABAQUS is shown in Figure 8.1. 


\subsection{Simulation Results}

The acoustic responses of steel plates and tubes are simulated in this project using ABAQUS. Figures $8.2-8.4$ show the Lamb wave propagation in a steel plate from 3.6663e-5 second to $1.1666 \mathrm{e}-4$ second. We can make several conclusions from these plots: first, the Lamb waves in plate like structures have a dominant anti-symmetric distribution with sine pulse activation on one side of the plate; second, the Lamb wave disperses due to the impurity of input signals. The system time response at a point in the plate is illustrated in Figure 8.5, where the amplitude of the particle displacement decreases with time. But, due to storage space limitation and computer memory, we were not able to simulate a large number of time steps. Therefore the material density and Poisson ratio are revised in $A B A Q U S$ ' input file to accelerate the wave propagation such that we could collect more information in a less number of time steps. So, the calculation of wave speed from Figure 8.5 is not included. However, we are still able to calculate the frequency response of the system as shown in Figure 8.6, where we find several resonant peaks around the active frequency of $27 \mathrm{kHz}$.

Figures 8.7 - 8.9 demonstrate the wave propagation in a tube from $3.9996 \mathrm{e}-5$ second to $6.9993 \mathrm{e}-4$ second. The observation shows that there are both symmetric and anti-symmetric modes in the tubular structure compared with only anti-symmetric mode in the plate. The contour plot of the tube shows the displacement of node points located on the tube surface.

Figures 8.11 and 8.11 show the time and frequency response of a tube structure to a sine pulse input.

A MATLAB code was created for the preparation of active input data files and for the post-processing of $A B A Q U S$ data file. The geometry inputs are generated using $A B A Q U S$ CAE pre-processor, and the plots of point displacement are from ABAQUS VIEW, a post-processing code. 


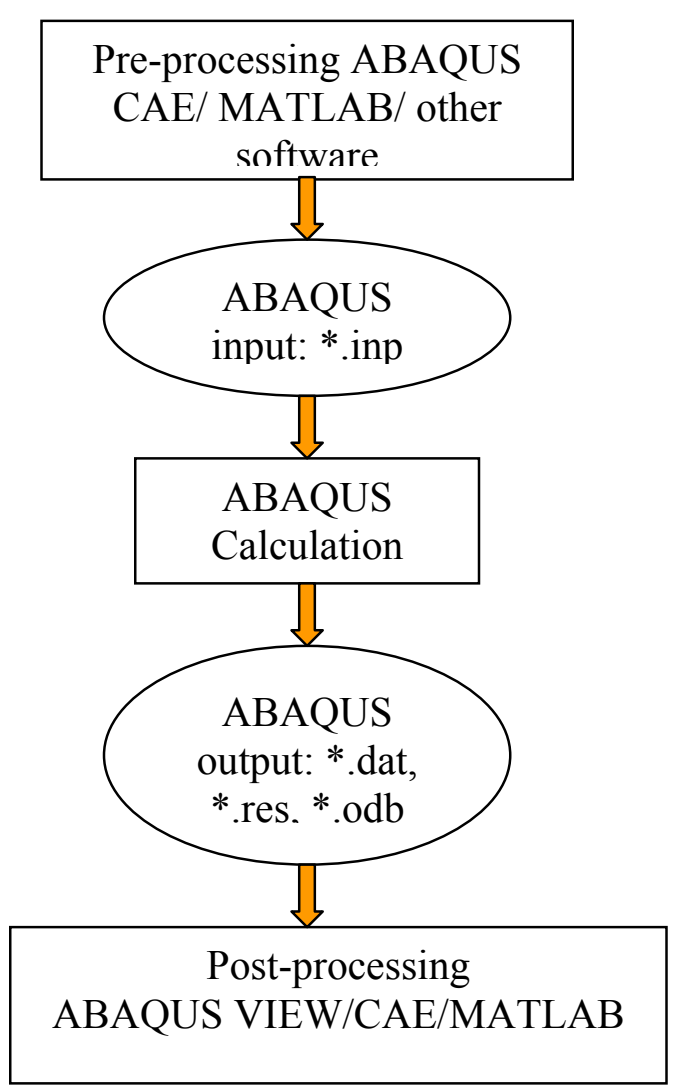

Figure 8.1. Flow chart for ABAQUS simulation process.
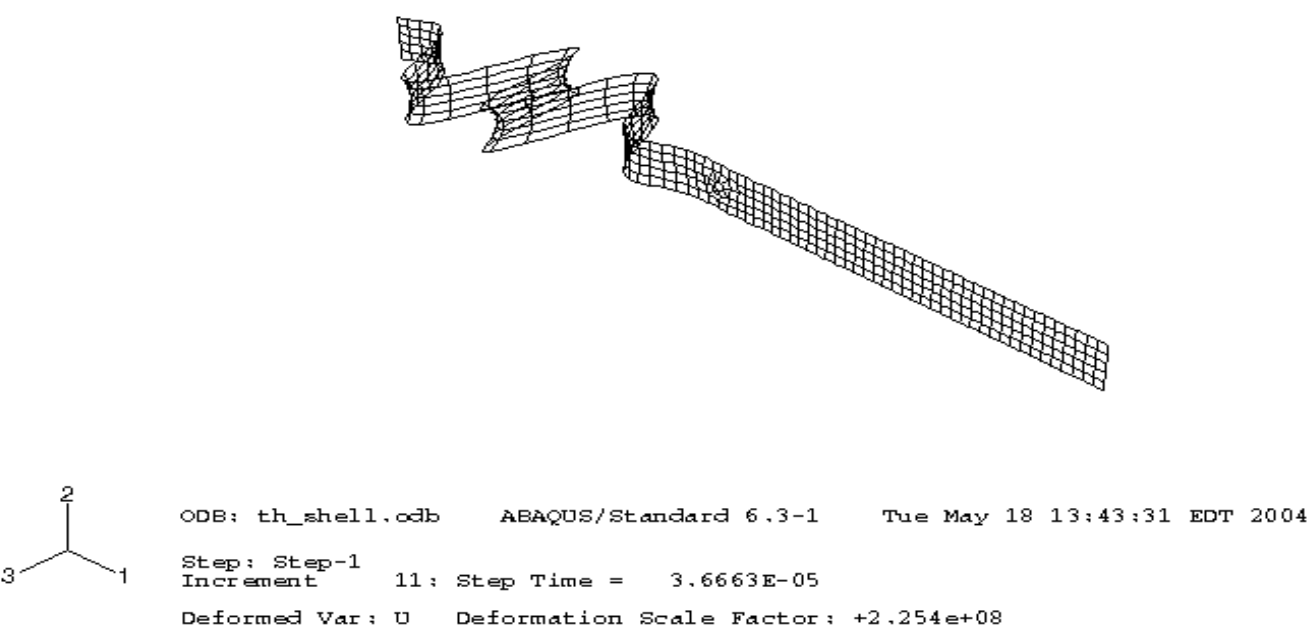

Figure 8.2. Lamb wave in a plate at time 3.6663e-5 second. 

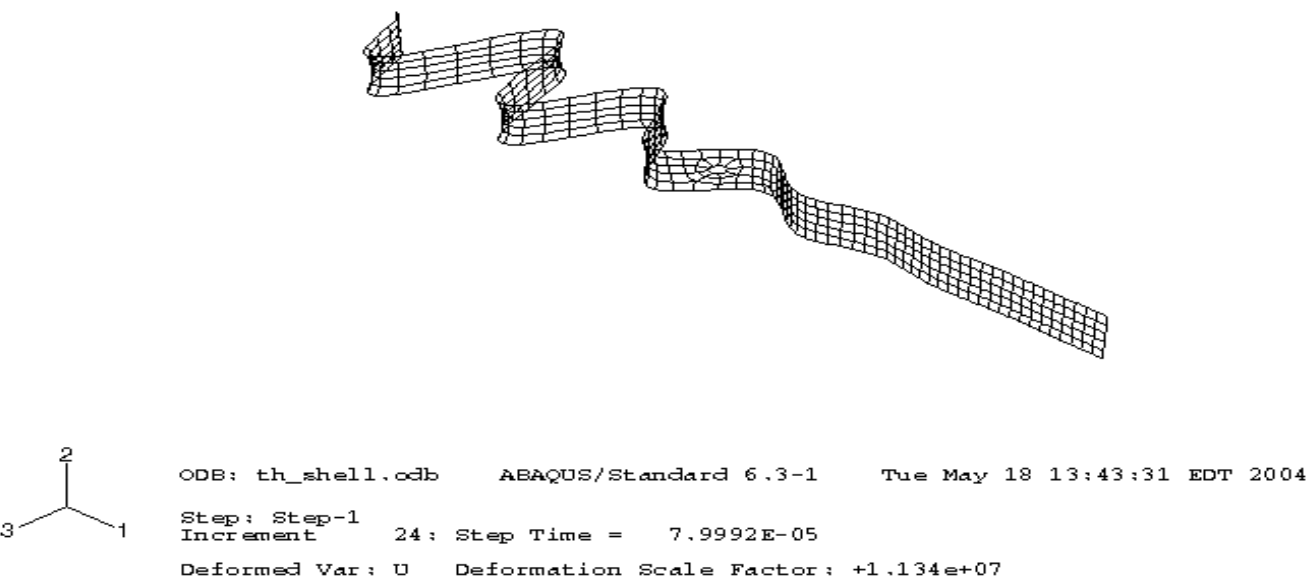

Figure 8.3. Lamb wave in a plate at time $7.9992 \mathrm{e}-5$ second.
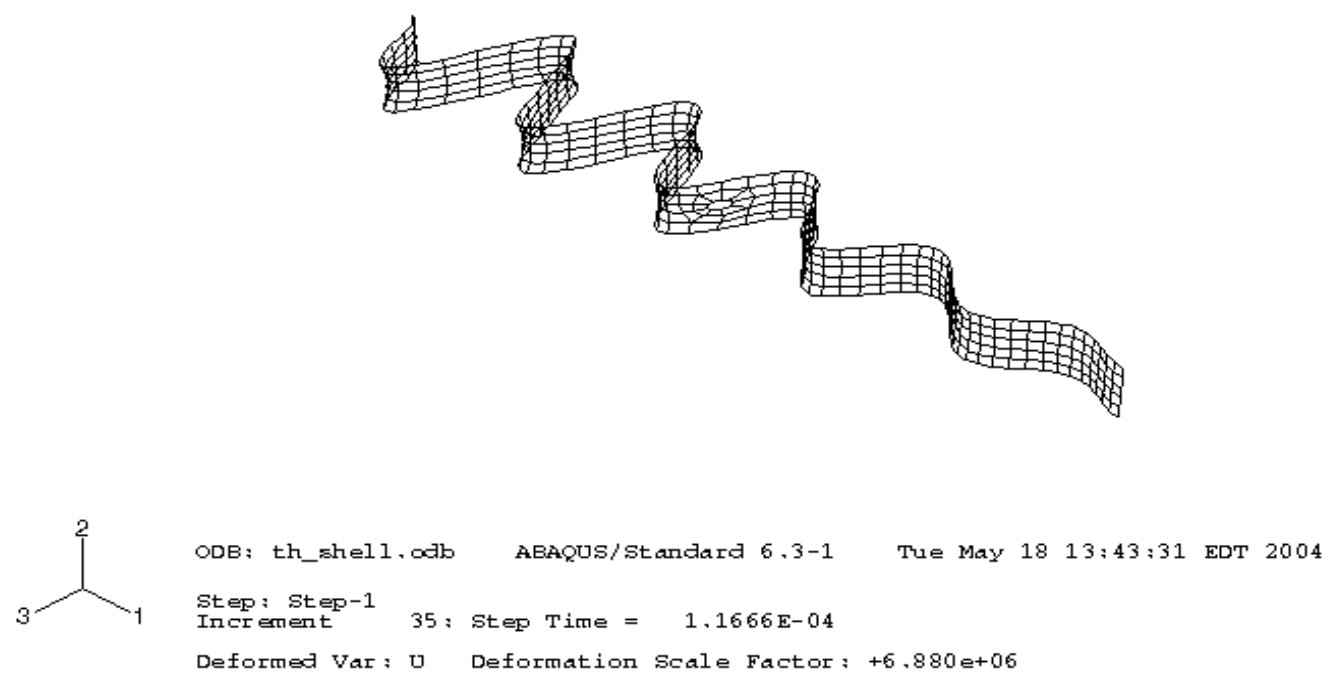

Figure 8.4. Lamb wave in a plate at time 1.1666e-4 second. 


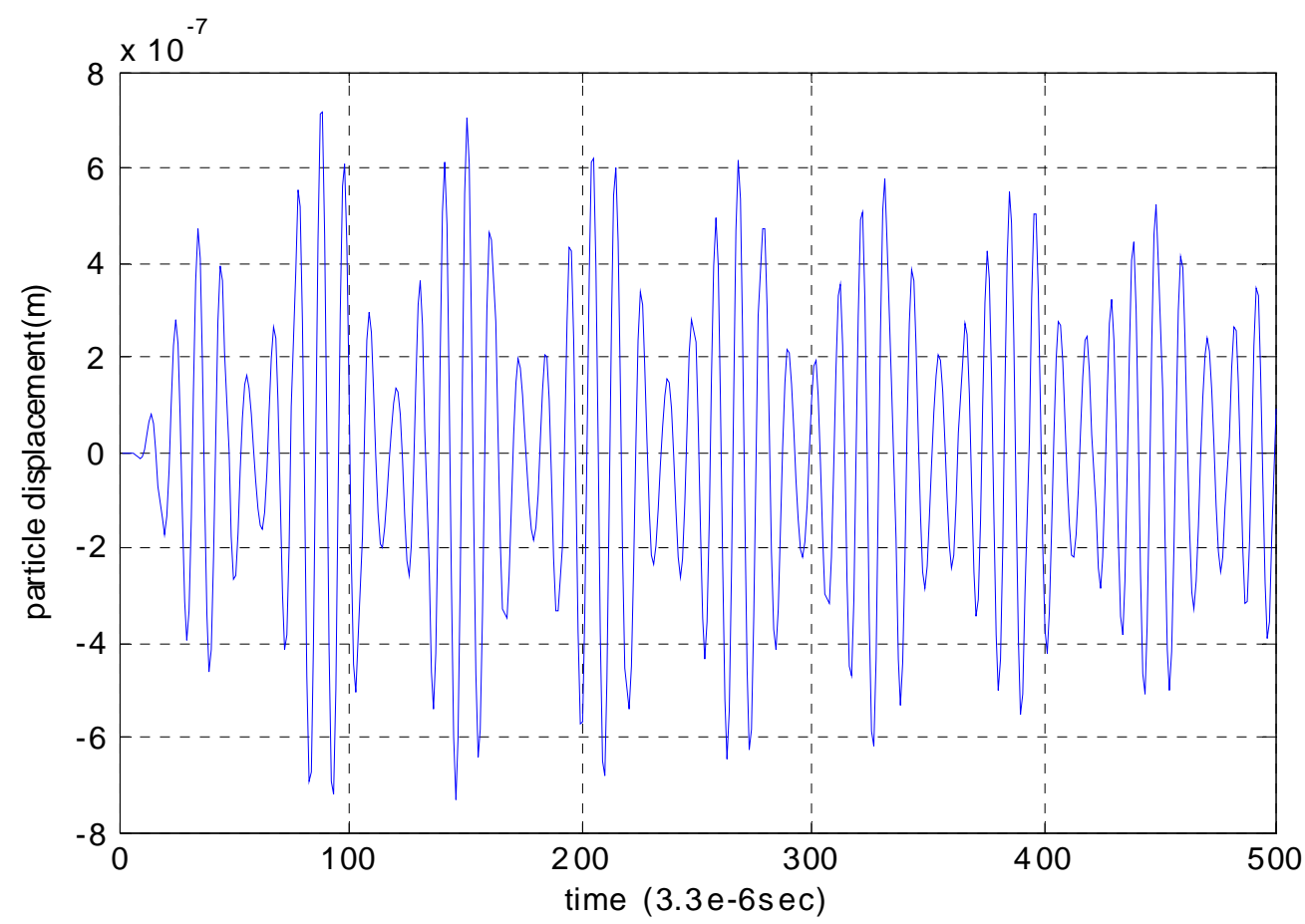

Figure 8.5. Particle displacement at one point on the plate.

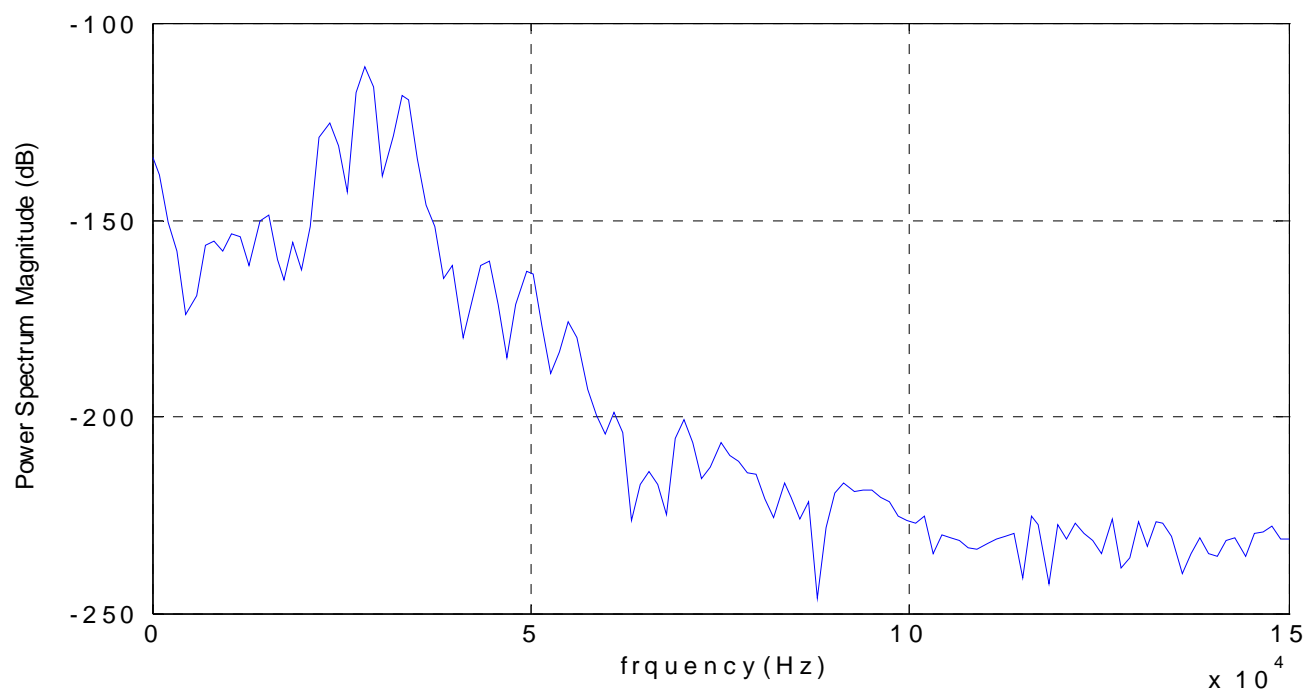

Figure 8.6. Frequency response for the plate (from Simulation). 


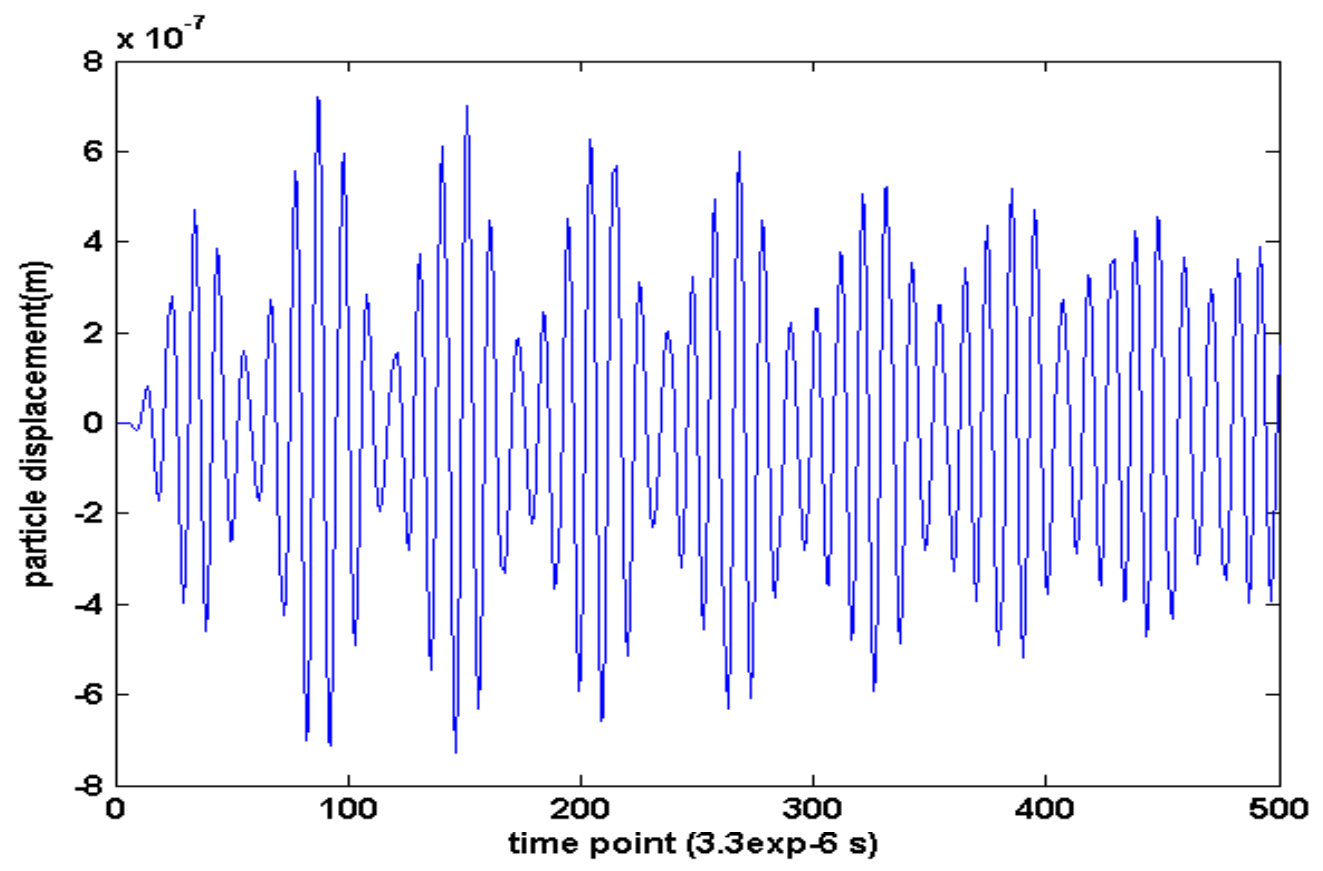

Figure 8.7. Particle displacement at one point on a normal plate. 


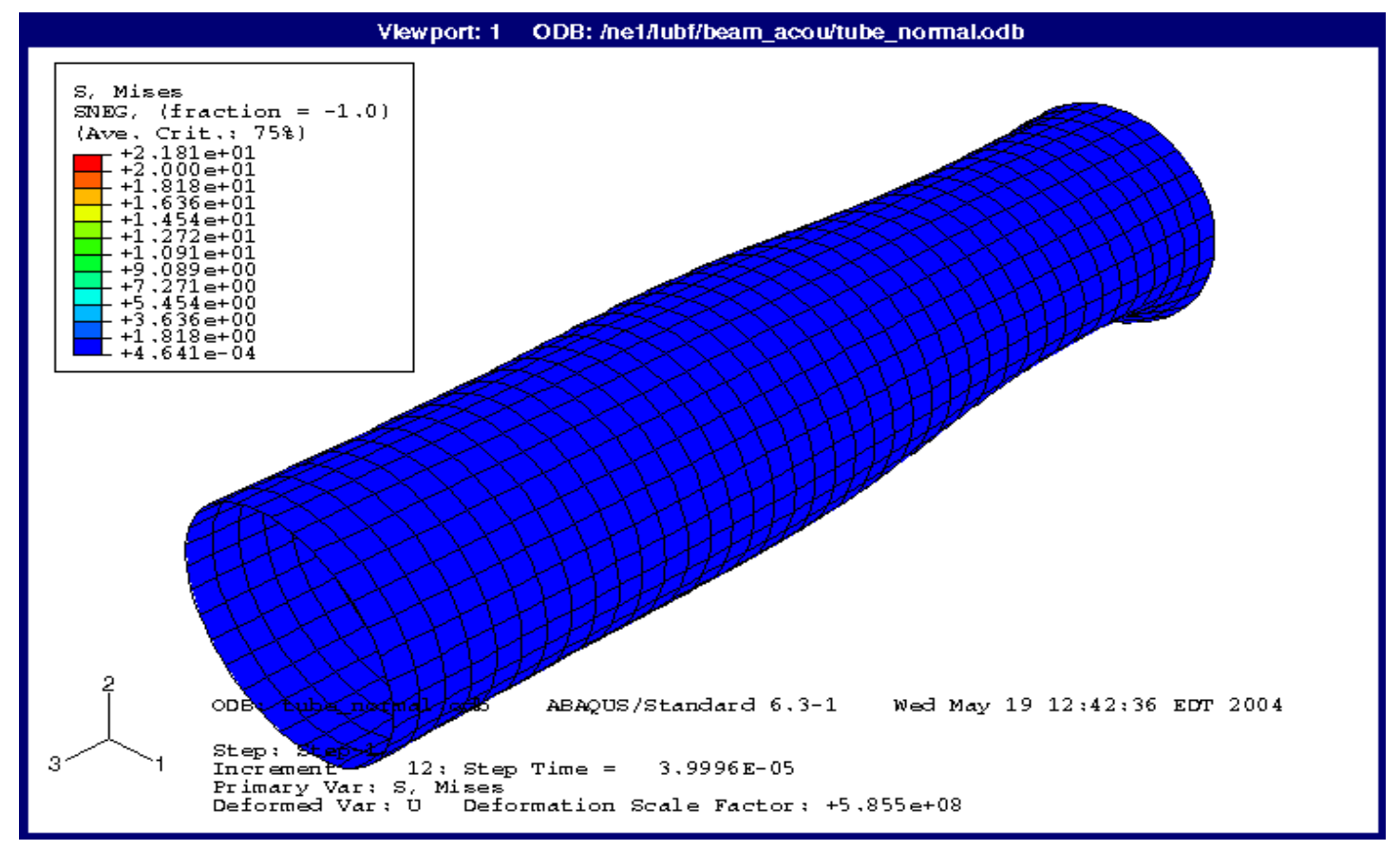

Figure 8.7. Lamb wave in a tube at time 3.9996e-5.

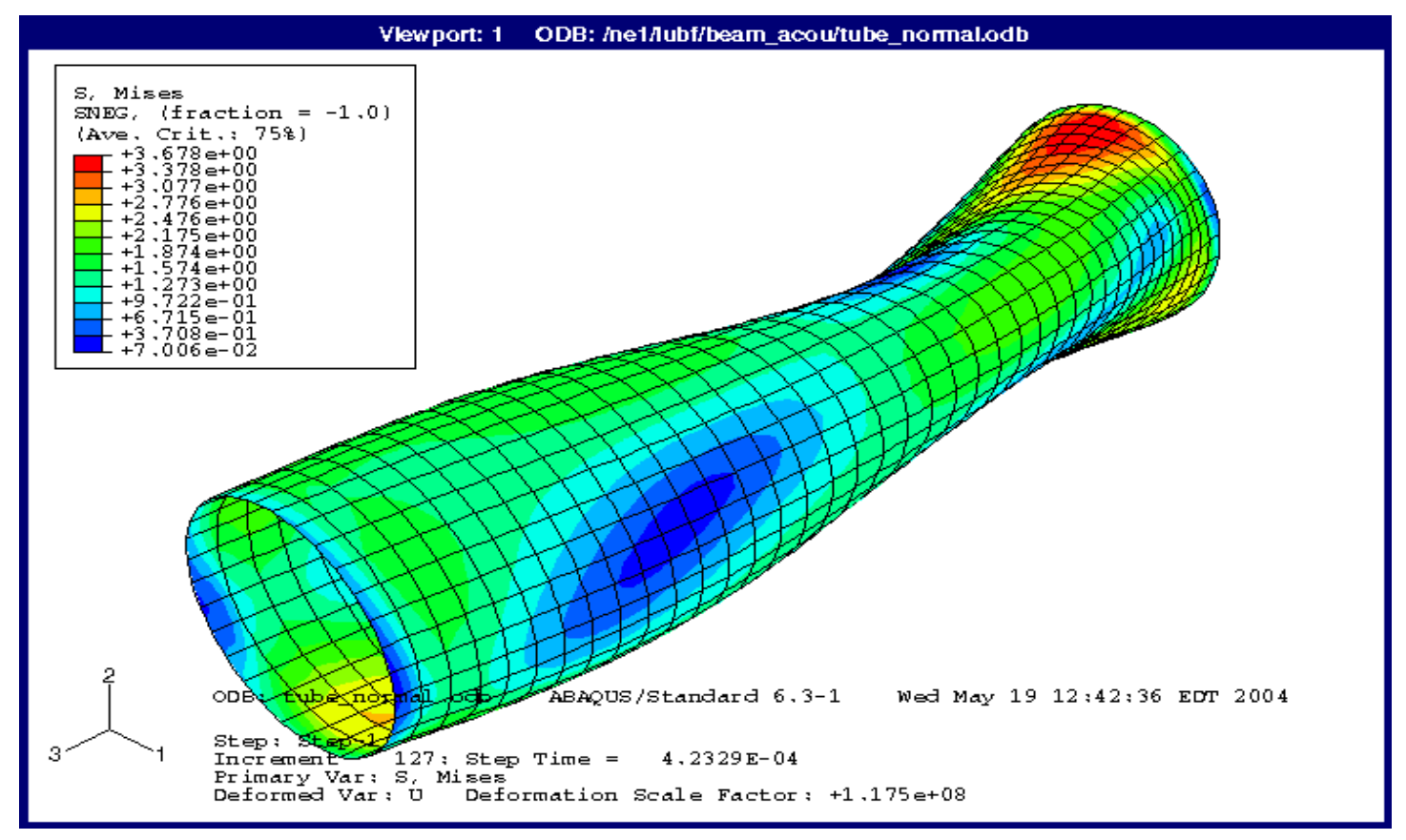

Figure 8.8. Lamb wave in a tube at time $4.2329 \mathrm{e}-4$. 


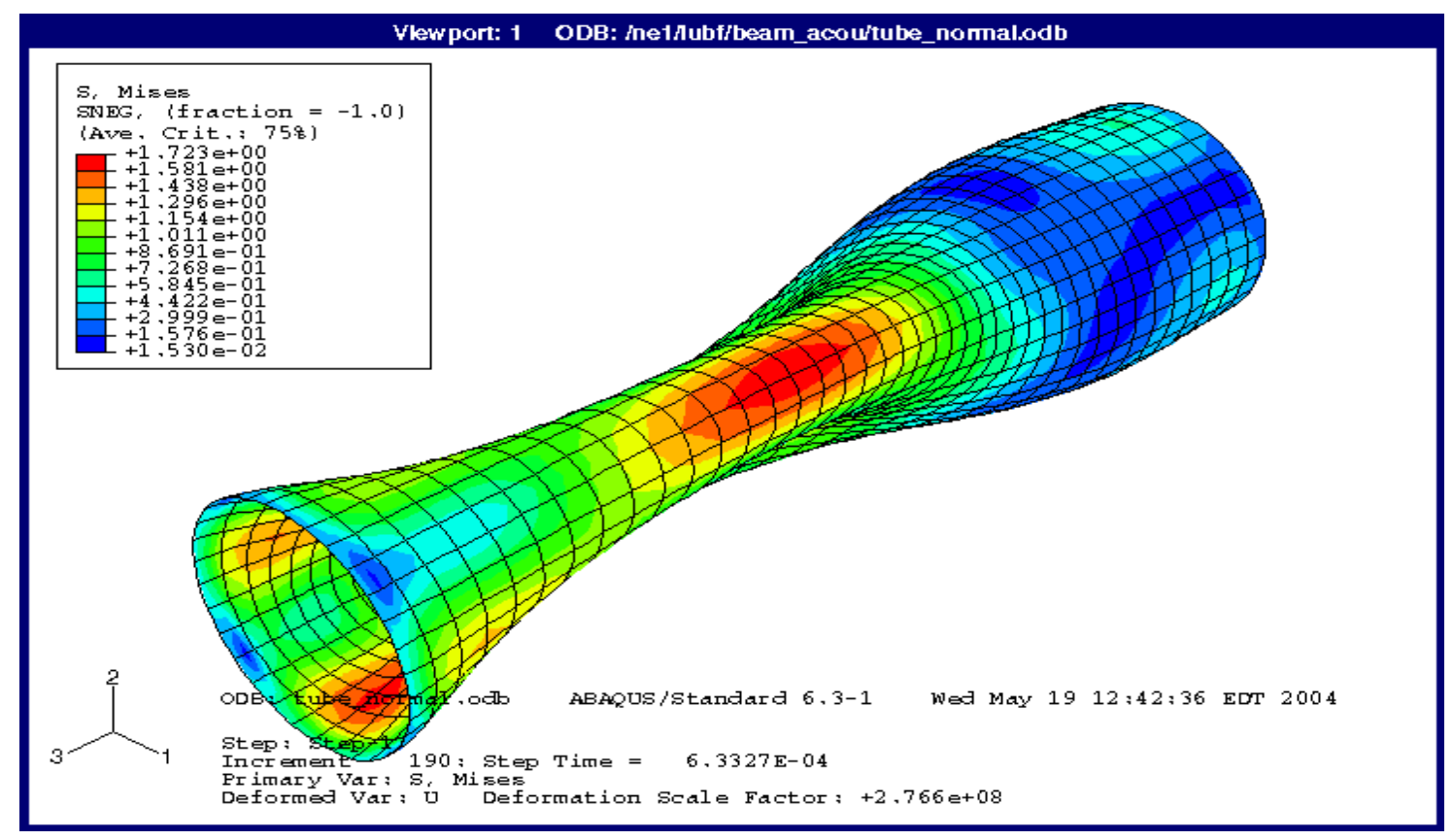

Figure 8.9. Lamb wave in a tube at time $6.3327 \mathrm{e}-4$.

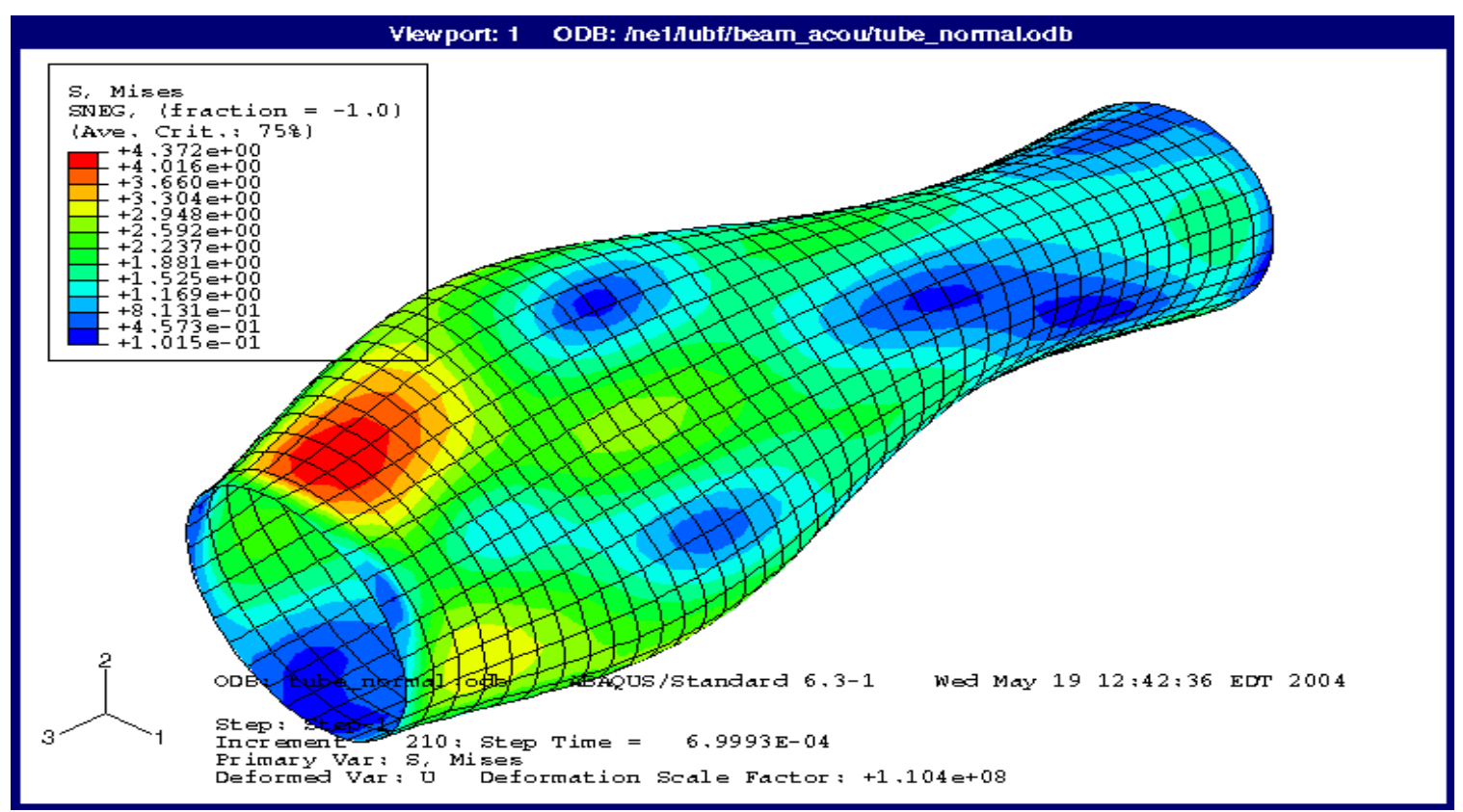

Figure 8.10. Lamb wave in a tube at time 6.9993e-4. 


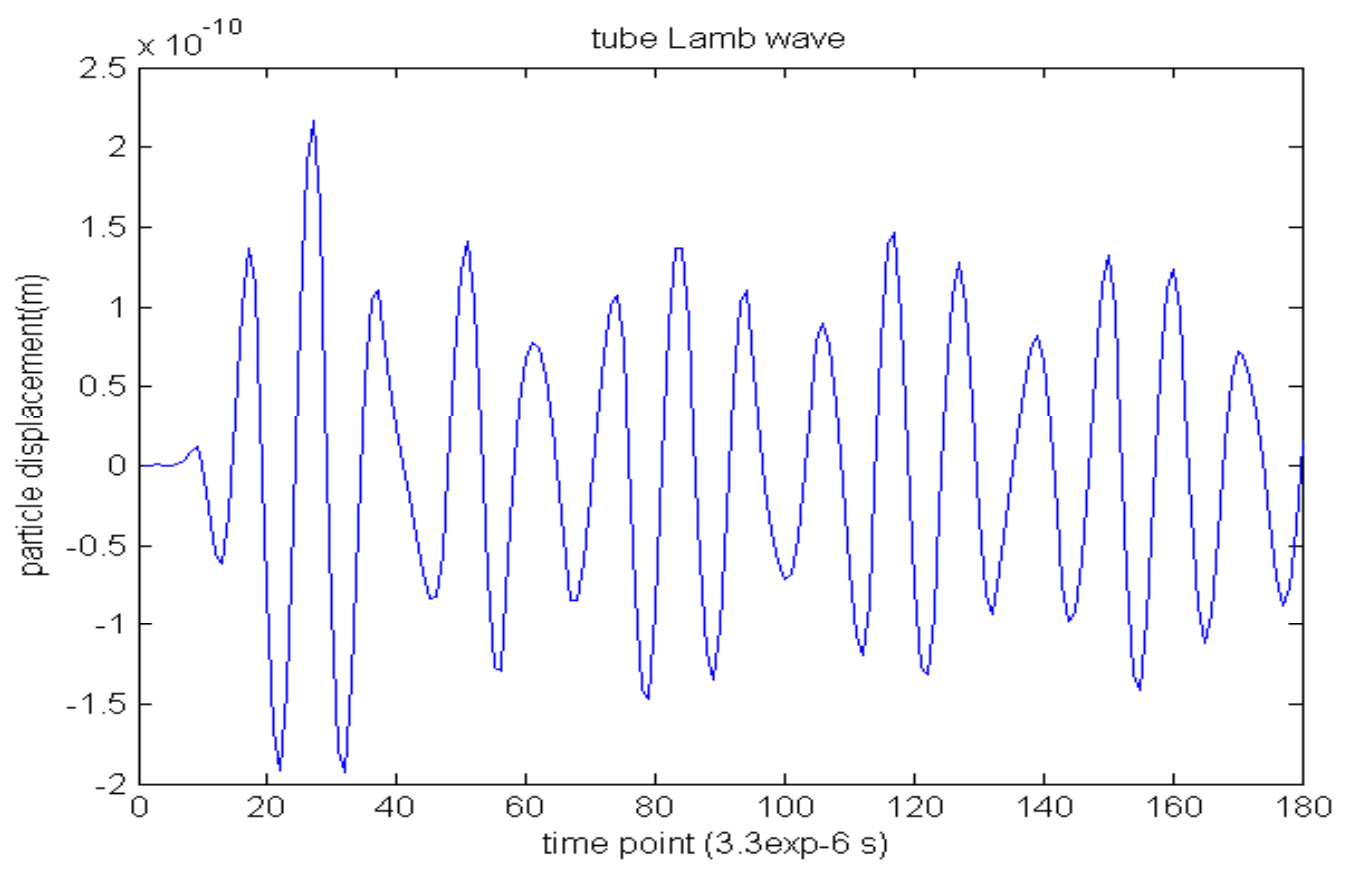

Figure 8.11. Particle displacement at one point on the plate.

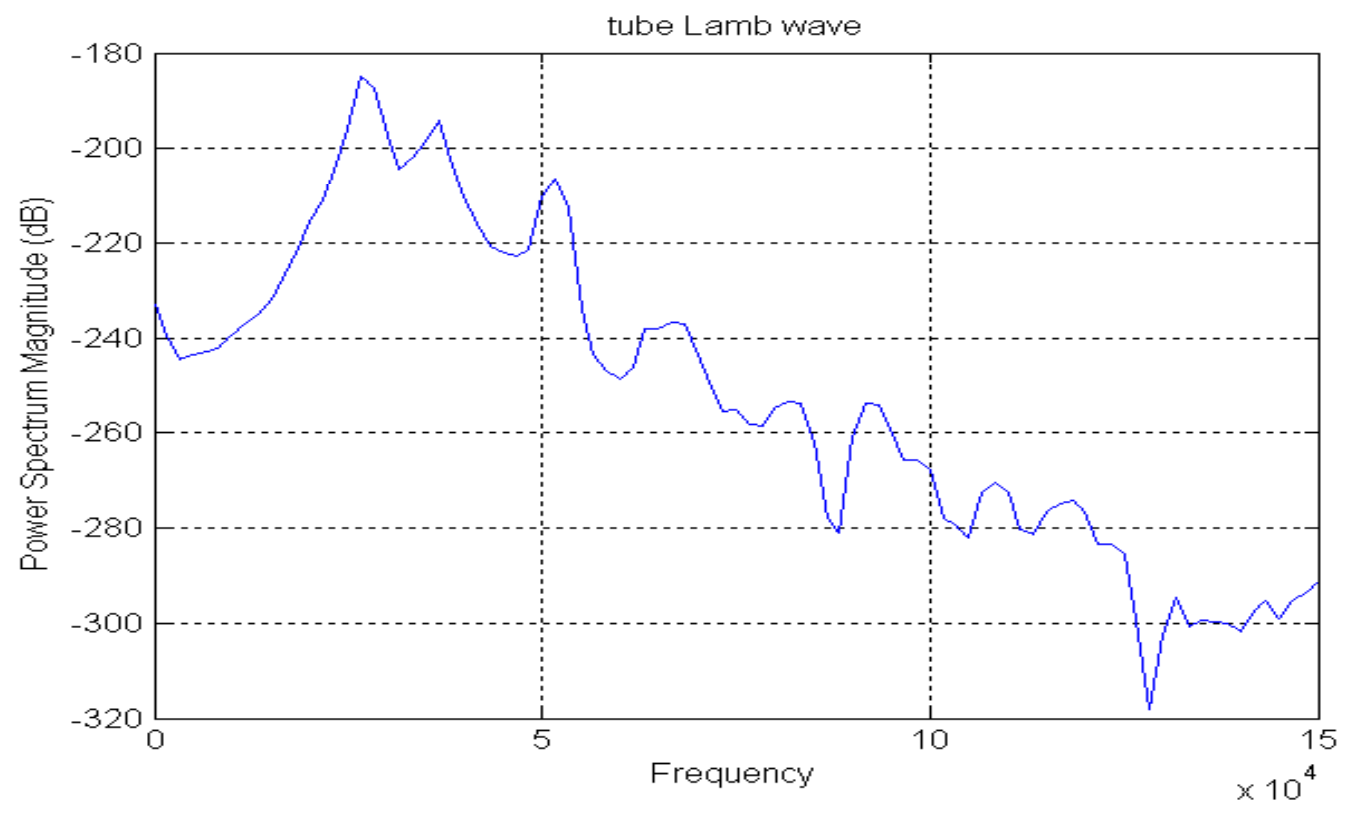

Figure 8.12. Frequency response for brass tube (from Simulation). 


\subsection{Concluding Remarks on Simulation Using ABAQUS}

Through the simulation results illustrated in the previous section, we found that the ABAQUS could be used to study the vibration of metal plates and tubes. The results verified that vibration frequency was decided by the excitation signals. The structural flaws affect the wave propagation along the structures. The simulation is also a good way to observe the dynamic process of wave propagation.

However, we also found many differences between the simulation and our experiments such as the signal amplitude and components. There are many reasons for these differences. The first is that elastic properties, i.e. the Lamb constants, were changed in the simulation inputs to accelerate wave propagation, because the source limit of the workstation where the ABAQUS is running. The large scale of the $3 \mathrm{D}$ wave simulation has fairly high requirement on computer storage, so the calculations must be limited to several hundred steps. A second reason is that a shell structure was used in plate and tube to simplify the geometry. This simplification may not be very suitable for the Lamb wave propagation, because the Lamb waves usually refer to the waves in a plate like or tubular structure with a small thickness. The third reason is that the boundary conditions may not be correctly simulated in ABAQUS, especially the leakage of energy to the air.

Despite all of these limitations, ABAQUS provided us with a convenient tool to study wave propagation. Some properties, especially the frequency response, can be clearly demonstrated. Further study of advanced simulation method is necessary for future research on the application of Lamb waves. 


\section{CONCLUSIONS AND SUGGESTIONS FOR FUTURE WORK}

\subsection{Conclusions}

From the study performed in this research, we conclude that Lamb waves used in this project are very sensitive to structural defects in brass tubes. The defect size, location, shape, and surrounding media are among the factors that affect the Lamb wave propagation along guided structures. The dimension of the smallest flaw illustrated in this study is about one millimeter. However, smaller defects including micro-structural variations could be monitored because the propagation of elastic waves strictly depends on the microstructure of materials.

Unfortunately, the Lamb wave is not as simple as the pure longitudinal and transverse wave that propagates along an infinite medium. The complexity comes from the combination of multi-mode waves for plate or tubular structures. There are four types of Lamb waves for tubular structures. For each mode, the wave speed decreases nonlinearly as the frequency-thickness product increases, accompanied by the appearance of new modes. Hence the features of experimental data collected from the sensor system cannot be easily visualized and analyzed. Non-stationary signal processing is important for acoustic data analysis in this research for the success of structural integrity diagnostics.

The structural flaw may change the characteristics in time and frequency domains. For this reason the Hilbert-Huang transform was introduced for its adaptiveness and sensitivity in separating wave modes, which was also found to be useful in filtering out noise due to water flow and voids. Two transient signal processing techniques, moving windows and zooming windows, are developed for the tubular acoustics activated by sine pulses. Good features were extracted for the detection, localization, size estimation, and classification of structural flaws.

An optimal setup was critical in collecting representative acoustic signals. A multi-sensor suite was deployed in a way that two sensors were 180-deg apart, such that the single mode Lamb wave signals could be separated. The separated Lamb waves were 
applied to the structural diagnostics of brass tubes. Excellent results were achieved by applying moving and zooming windows to the separated signals.

The methods of transient data analysis were proved to be effective for the brass tubes immersed in the water and under the effect of two-phase flow. The HHT was successfully implemented for noise reduction. A direct effect of submerging in water on the Lamb waves in tubing was the increase of energy leakage.

There should be potentially a wide range of applications of this technique in the aerospace and other industries. The techniques developed here are especially useful for the on-line in-situ monitoring of key equipment to improve system reliability and safety.

\subsection{Suggestions for Future Work}

Because of the potentially large number of applications of the guided acoustic techniques, further research must be performed to develop industrial implementation of this method. There are many interesting and challenging problems in the areas of acoustic sources, acoustic sensors, and acoustic signal analysis. Some suggestions are given below.

First, a detailed study of the effect of high temperature and high pressure on the performance of piezoelectric sensors should be performed before the implementation of the techniques described in this research for steam generator tubes and other equipment under hostile environment. Underwater signals have a significant bias due to the pressure of water.

Second, a good numerical solution algorithm is desirable in the future study for theoretical understanding of acoustic leakage from elastic media to a liquid such as water. Acoustics propagation along a buried tube is also a very interesting topic.

Third, the techniques developed in this research may be expanded to the structural monitoring of large dimension pipes. Due to the large dimension of pipes, better results are expected. Large-scale experimental data are necessary for analysis following the theoretical study mentioned above. A comb-like active sensor should be further investigated in generating acoustics with selected wavelength. 
Finally, the application of photo-acoustics for microstructure study is very applicable in the material evaluation and underwater studies. Photo-acoustic means generating acoustics waves through intensive non-mechanical waves, such as lasers, Xrays, and others. The laser generated Lamb waves must be further studies for practical applications.

In summary, the future work should focus on extending the current techniques developed in this research to a wide range of applications, addressing practical problems such as the effect of high temperature on piezo-devices, and exploring new methods for acoustic signal generation and information extraction. 


\section{BIBLIOGRAPHY}

1. B. Lu, B.R. Upadhyaya and J.W. Hines, " Time-frequency Analysis of Acoustic Signals for Flaw Monitoring in Steam Generator Structures," MRC, May 2003

2. A.A. Oliner et al., Acoustic Surface Waves, Springer-Verlag, Berlin, 1978.

3. B.A. Auld, Acoustic Fields and Waves in Solids, $2^{\text {nd }}$ Edition, Robert E. Krieger Publishing Company, 1990.

4. C. A. Paget, S. Grondel, K. Levin, and C. Delebarre, "Damage Assessment in Composites by Lamb Waves and Wavelet Coefficients," Smart Materials and Structures 12, pp.393-402, 2003.

5. C. Campbell, Surface Acoustic Wave Devices and Their Signal Processing Applications, Academic Press, New York, 1989.

6. C. Chen, C. Li, T. Teng, "Surface-Wave Dispersion Measurement Using HilbertHuang Transform," TAO, Vol.13, No.2, pp.171-184, June 2002.

7. C. Valle and J. Littles Jr, "Flaw Localization Using the Reassigned Spectrogram on Laser-generated and Detected Lamb Modes," Ultrasonics 39, pp. 535-542, 2002.

8. C.H. Chen, Issues in Acoustic Signal Image Processing and Recognition, SpringerVerlag, Berlin, 1983.

9. C.K. Chui, An Introduction to Wavelets, Academic Press, New York, 1992.

10. D Alleyne, M. Lowe, and P. Cawley, "The Reflection of Guided Waves From Circumferential Notches in Pipes," J. of Applied Mechanics, Vol. 65, pp.635-641, Sep. 1998.

11. D. Alleyne and P. Cawley, "A Two-dimensional Fourier Transform Method for the Measurement of Propagating Multimode Signals," J. Acoust. Soc. Am., Vol. 89, No. 3, pp. 1159-1167, March 1991.

12. D.F. Parker and G.A. Maugin (Eds), Recent Developments in Surface Acoustic Waves, Springer-Verlag, New York, 1987.

13. D.N. Alleyne, M.J.S. Lowe and P. Cawley, "The Reflection of Guided Waves From Circumferential Notches in Pipes," J. of Applied Mechanics, Vol. 65, pp. 635-641, September 1998.

14. D.S. Ballantine et al., Acoustic Wave Sensors Theory, Design and PhysicalChemical Applications, Academic Press, San Diego, 1997.

15. E. Clezio, M. Castaings, and B. Hosten, "The Interaction of the $\mathrm{S}_{0}$ Lamb Mode With Vertical Cracks in an Aluminum Plate", Ultrasonics 40, pp. 187-192, 2002.

16. E.V. Malyarenko and M.K. Hinders, "Fan Beam and Double Cross-hole Lamb Wave Tomography for Mapping Flaws in Aging Aircraft Structures," J. of Acoust. Soc. Am., Vol. 108, No. 4, pp. 1631-1639, October 2000.

17. F.B. Stumpf, Analytical Acoustics, Ann Arbor Science Publishers, Michigan, 1980.

18. G. Kino, "The Application of Reciprocity Theory to Scattering of Acoustic Waves by Flaws", J. Applied Physics, Vol. 49, No.(6), pp. 3190-3199, June 1978.

19. G. Strang and T. Nguyen, Wavelets and Filter Banks, Wellesley-Cambridge Press, 1997.

20. G.C. Gaunaurd, "Elastic and Acoustic Resonance Wave Scattering," Applied Mechanics Review, Vol. 42, No. 6, pp. 143-192, June 1989. 
21. G.V. Frisk, J.W. Dickey, and H. Uberall, "Surface Wave Modes on Elastic Cylinders," J. Acoust. Soc. Am., Vol.58, No. 6, pp. 996-1008, November 1975.

22. I.A. Viktorov, Raleigh and Lamb Waves, Plenum Press, New York, 1967.

23. J.C.P. McKeon and M.K. Hinders, "Lamb Wave Scattering From A Through Hole," J. of Sound and Vibration, 224(5), pp.843-862, 1999.

24. J. Barshinger, J.L. Rose, and M.J. Avioli, "Guided Wave Resonance Tuning for Pipe Inspection," J. of Pressure Vessel Technology, Vol. 124, pp. 303-310, August 2002.

25. J. L. Rose, "A Baseline and Vision of Ultrasonic Guided Wave Inspection Potential," J. of Pressure Vessel Technology, Vol. 124, pp. 273-282, August 2002.

26. J. Miklowitz, The Theory of Elastic Waves and Waveguides, North-Holland Publishing Company, New York, 1978.

27. J. N. Yang, S. Lin, and S. Pan, "Damage Identification of Structures Using HilbertHuang Spectral Analysis," $15^{\text {th }}$ ASCE Engineering Mechanics Conference, June 2$5,2002$.

28. J.J. Ditri, "Utilization of Guided Elastic Waves for the Characterization of Circumferential Cracks in Hollow Cylinders," J. Acoust. Soc. Am., Vol. 96, No. 6, pp. 3769-3775, December 1994.

29. J.L. Davis, Wave Propagation in Solids and Fluids, Springer-Verlag, New York, 1988.

30. J.L. Rose, Ultrasonic Waves in Solid Media, Cambridege University Press, 1999.

31. K. Motegi, "Ultrasound Radiation into Water by a Lamb Wave Device Using a Piezoelectric Ceramic Plate with Spatially Varying Thickness," Ultrasonics, Vol. 37, pp. 505-510, 1999.

32. K.F. Graff, Wave Motion in Elastic Solids, Ohio State University Press, 1975.

33. L. Cohen, Time-Frequency Analysis, Prentice Hall, 1995.

34. L.B. Felsen et al., Transient Electromagnetic Fields, Springer-Verlag, New York, 1976.

35. L.E. Kinsler and A.R. Frey, Fundamentals of Acoustics, $2^{\text {nd }}$ Edition, John Wiley, 1962.

36. L.R. Dragonette, D.M. Drumheller, C.F. Gaumond, D.H. Hughes, B.T. O’Connor, N. Yen, and T.J. Yoder, "The application of Two -Dimensional Transformations to the Analysis and Synthesis of Structural Excitations Observed in Acoustical Scattering," Proceedings of the IEEE, Special Issue on Time Frequency Analysis, Vol. 84, No. 9, pp. 1249-1263, September 1996.

37. M. Deng, "Cumulative Second-harmonic Generation of Generalized Lamb-wave Propagation in a Solid Wave-guide, J. Appl. Physics, Vol. 33, pp. 207-215, 2000.

38. M. Niethammer and L. Jacobs, "Time-frequency Representations of Lamb Waves", J. Acoust. Soc. Am., Vol. 109, No. 5, pp. 1841-1847, December 1994.

39. M. Salvetti, G. Lombardi, and F. Beux, "Application of a Wavelet CrossCorrelation Technique to the Analysis of Mixing," AIAA Journal, Vol.37, No.8, pp.1007-1009, Aug. 1999.

40. M. Tohyama and T. Koike, Fundamentals of Acoustic Signal Processing, Academic Press, 1998. 
41. M.E. Montesinos, J.L. Munoz-Cobo and C. Perez, "Hilbert-Huang Analysis of BWR Neutron Detector Signals: Application to DR Calculation and to Corrupted Signal Analysis," Annals of Nuclear Energy 30, pp.715-727, 2003.

42. N. Yen, "Wave Packet Decomposition," J. Acoust. Soc. Am., Vol. 95, No. 2, pp. 889-896, February 1994.

43. N.E. Huang et. al, "The Empirical Mode Decomposition and the Hilbert Transformation for Nonlinear and Non-stationary time series analysis," Proc. R. Soc. Lond. A 454, pp.903-995, 1998.

44. O.V. Rudenko and S.I. Soluyan (Translated by R. T. Beyer), Theoretical Foundations of Nonlinear Acoustics, Consultants Bureau, New York, 1977.

45. P. Fromme and M.B. Sayir, "Measurement of The Scattering of A Lamb Wave by A Through Hole In a Plate," J. Acoust. Soc. Am., 111(3), pp. 1165-1170, March 2002.

46. P. Maass, G. Teschke, W. Willmann, and G. Wollmann, "Detection and Classification of Material Attributes - A Practical Application of Wavelet Analysis, IEEE Transactions on Signal Processing," Vol. 48, No. 8, August 2000.

47. P.J. Oonincx, Mathematical Signal Analysis: Wavelets, Wigner Distribution, and a seismic Application, Stichting Mathematisch Centrum, Amsterdam, 2000.

48. R. Carmona, W. Hwang, and B. Torresani, Practical Time-Frequency Analysis, Academic Press, New York, 1998.

49. R.R. Zhang, S. Ma, E. Safak, and S. Hartzell, " Hilbert-Huang Transform Analysis of Dynamic and Earthquake Motion Recordings," Journal of Engineering Mechanics, pp.861-875, 2003.

50. S. Nettel, Wave Physics, $2^{\text {nd }}$ Edition, Springer, New York, 1995.

51. S. Quek, O. Tua, and Q. Wang, "Detecting Anomalies in Beams and Plate Based on the Hilbert-Huang Transform of Real Signals," Smart Materials and Structures 12, pp.447-460, 2003.

52. S. S. Kessler, Piezoelectric-Based In-Situ Damage Detection of Composite Materials for Structural Health Monitoring Systems. PhD thesis, MIT, 2002.

53. S.K. Goumas, M.E. Zervakis, and G.S. Stavrakakis, "Classification of Washing Machines Vibration Signals Using Discrete Wavelet Analysis for Feature Extraction, IEEE Transactions on Instrumentation and Measurement, Vol. 51, No. 3, June 2002.

54. T. Ghosh, T. Kundu and P. Karpur, "Efficient Use of Lamb Modes for Detecting Defects in Large Plates," Ultrasonics 36, pp. 791-801, 1998.

55. T.W. Wu, "On Computational Aspects of the Boundary Element Method for Acoustic Radiation and Scattering in a Perfect Waveguide, J. Acoust. Soc. Am., Vol. 96, No. 6, pp. 3733-3743, December 1994.

56. U.B. Halabe and R. Franklin, "Fatigue Crack Detection in Metallic Members Using Ultrasonic Rayleigh Waves with Time and Frequency Analysis," Materials Evaluation, Vol. 59, No. 3, pp. 424-431, March 2001.

57. V. Agostini, P.P. Delsanto, and I. Genesio "Simulation of Lamb Wave Propagation in Composite Materials," Science and Supercomputing at CINECA, Report 2001.

58. V. Pagneux and A. Maurel, "Determination of Lamb Mode Eigenvalues," J. Acoust. Soc. Am., 110(3), pp.1307-1314, Sep. 2001. 
59. V.P. Gilbert, Surface Acoustic Wave Device Designed to Monitor Frequency Shifts due to Adsorption of Mass on to a Piezoelectric Crystal, M.S. Thesis, University of Tennessee, May 1992.

60. Y. Jung, T. Kundu, and M. Ehsani, "Internal Discontinuity Detection in Concrete By Lamb Waves", Materials Evaluation, Vol. 59, No. 3, pp. 418-423, March 2001.

61. T. Kaewkongka, Y.H. J. Au, R. Rakowski, and B.E. Jones, "Continuous Wavelet Transform and Neural Network for Condition Monitoring of Rotodynamic Machinery", IEEE instrumentation and Measurement, Technology conference, Budapest, Hungary, pp.1962-1966, May 2001.

62. H. Farid and E. H. Adelson, "Separating Reflections From Images by Use of Independent Component Analysis", Journal of Optical Society of America, Vol.16, No.9, pp.2136-2145, September 1999.

63. M. Al-aghbari, F. Scarpa and W. J. Staszewski, "On the Orthogonal Wavelet Transform for Model Reduction/Synthesis of Structures", Journal of Sound and Vibration, 254(4), pp. 805-817, 2002.

64. G. Barbastathis, and D. J. Brady, "Multidimensional Tomographic Imaging Using Volume Holography", Proceedings of The IEEE, Vol.87, No.12, pp.2098-2120, December 1999.

65. L. Wang, J. Zhang, C. Wang, and S. Hu, "Identification of Nonlinear Systems Through Time-frequency Filtering Technique", Journal of Vibration and Acoustics, Vol.125, pp.199-204, April 2003.

66. C. Hory, N. Martin, and A. Chehikian, "Spectrogram Segmentation by Means of Statistical Features for Non-stationary Signal Interpretation", IEEE Transactions on Signal Processing, Vol.50, No.12, pp.2915-2925, December 2002.

67. L. Durak, and O. Arikan, "Short-Time Fourier Transform: Two Fundamental Properties and An Optimal Implementation", IEEE Transactions on Signal Processing, Vol.51, No.5, pp.1231-1242, May 2003.

68. M. Browne, S. Shiry, M. Dorn, and R. Ouellette, "Visual Feature Extraction via PCA-based Parameterization of Wavelet Density Functions", International Symposium on Robots and Automation, 2002.

69. B. Hu and R. G. Gosine, "A New Eigenstructure Method for Sinusoidal Signal Retrieval in White Noise: Estimation and Pattern Recognition", IEEE Transactions on Signal Processing, Vol.45, No.12, pp.3073-3083, December 1997.

70. H. Sohn, C. R. Farrar, M. L. Fugate, and J. J. Czarnecki, "Structural Health Monitoring of Welded Connections", The First International Conference on Steel \& Composite Structures, Pusan, Korea, June 2001.

71. P. N. Belhumeur, J. P. Hespanha, and D. J. Kriegman, “Eigenfaces vs. Fisherfaces: Recognition Using Class Specific Linear Projection", IEEE Transactions on Pattern Analysis and Machine Intelligence, Vol.19, No.7, pp.711-720, July 1997.

72. M. J. Lyons, J. Budynek, and S. Akamatsu, "Automatic Classification of Single Facial Images", IEEE Transactions on Pattern Analysis and Machine Intelligence, Vol.21, No.12, pp.1357-1362, December 1999.

73. R. W. Glenny, H. T. Robertson, S. Yamashiro, and J. B. Bassingthwaighte, "Applications of Fractal Analysis to Physiology", Brief Review, the American Physiological Society, 2001. 
74. Y. Tian, T. Kanade, and J. F. Cohn, "Recognizing Action Units for Facial Expression Analysis", IEEE Transactions on Pattern Analysis and Machine Intelligence, Vol.23, No.2, pp.97-115, February 2001.

75. R. Feraud, O. J. Bernier, and M. Collobert, “A Fast and Accurate Face Detector Based on Neural Networks", IEEE Transactions on Pattern Analysis and Machine Intelligence, Vol.23, No.1, pp.42-53, January 2001.

76. M. C. Wellman, N. Srour, and D. B. Hillis, "Feature Extraction and Fusion of Acoustic and Seismic Sensors for Target Identification”, SPIE, Vol.3081, pp.139$145,1997$.

77. C. Liu, and H. Wechsler, "Evolutionary Pursuit and Its Application to Face Recognition", IEEE Transactions on Pattern Analysis and Machine Intelligence, Vol.22, No.6, pp.570-582, June 2000.

78. W. Chou and B.H. Juang, Pattern Recognition in Speech and Language Processing, CRC Press, Washington D.C., 2003.

79. G. Kawiecki and A. Seagle, "Feasibility of Applying Distributed Piezotransducers to Structural Damage Detection," Proceedings of MARCON 98, Vol. 2, pp. 69.0169.11, May 1998.

80. M. Kirby, Geometric Data Analysis: An Empirical Approach to Dimensionality Reduction and the Study of Patterns, John Wiley \& Sons, INC., New York, 2001.

81. H. S. Baird, Model-based Image Matching using Location, The MIT press, Massachusetts, 1985.

82. E. Grall-Maes and P. Beauseroy, "Mutual Information-Based Feature Extraction on the Time-Frequency Plane”, IEEE Transactions on Signal Processing, Vol.50, No.4, pp.779-790, April 2002.

83. Z. Peng, F. Chu, and Y. He, "Vibration Signal Analysis and Feature Extraction Based On Reassigned Wavelet Scalogram", Journal of Sound and Vibration, 253(5), pp.1087-1100, 2002.

84. Z. Peng, Y. He, and F. Chu, "Feature Extraction Of the Rub-Impact Rotor System By Means of Wavelet Analysis", Journal of Sound and Vibration, 259(4), pp.10001010, 2003.

85. Q.Q. Huynh, L. N. Cooper, N. Intrator, and H. Shouval, "Classification of Underwater Mammals Using Feature Extraction Based On Time-Frequency Analysis and BCM Theory", IEEE Transactions on Signal Processing, Vol.46, No.5, pp.1202-1207, May 1998.

86. R. L. Kettig and D.A. Landgrebe, "Classification of Multi-spectral Image Data By Extraction and Classification of Homogeneous Objects", IEEE Transactions on Geoscience Electronics, Vol.GE-14, No.1, pp.19-26, January 1976.

87. J. Zelenka, "Piezoelectric Resonators and their Applications," Elsevier Science, October 1986.

88. B. Lu, Development of a Fault Detection and Isolation Method for a Nuclear Power Plant Steam Generator System, M.S. thesis, University of Tennessee, 2002.

89. D. C. Gazis, "Exact Analysis of the Plane-Strain Vibrations of Thick-Walled Hollow Cylinders”, J. Acoust. Soc. Am., Vol. 30, No. 8, pp. 786-794, August 1958.

90. R.M. Cooper and P.M. Naghdi, "Propagation of Nonaxially Symmetric Waves in Elastic Cylindrical Shells”, J. Acoust. Soc. Am., Vol. 29, No. 12, pp. 1365-1373, December, 1957. 
91. G. M. Daskalov, Propagation of Ultrasonic Pulses in Flawed Media: Application to Nondestructive Testing and Evaluation, Ph.D. Dissertation, University of Tennessee, 1996.

92. A. E. Armenakas, D. C. Gazis, and G. Herrmann, Free Vibrations of Circular Cylindrical Shells, PERGAMON Press, New York, 1969.

93. A. Springer, R. Weigel, A. Pohl, and F. Seifert, "Wireless Identification and Sensing Using Surface Acoustic Wave Devices", PERGAMON, Mechatronics, No. 9, pp.745-756, 1999.

94. U. Grenander, Elements of Pattern Theory, The Johns Hopkins University Press, London, 1996.

95. http://himalaya.tamu.edu/help/softwareDocs/abaqus63/, 2004.

96. K. Uchino, Piezoelectric Actuators and Ultrasonic Motors, Kluwer Academic Publishers, November 1996.

97. M.E. Regelbrugge, "Smart Structures and Integrated Systems", Vol. 332, SPIEInternational Society for Optical Engineering, July 1998.

98. C.S. Lynch, "Smart Structures and Materials 2000 Active Materials: Behavior and Mechanics,” SPIE Optical Engineering Press, January 2000.

99. J. Holnicki-Szulc et al., "Smart Structures : Requirements and Potential Applications in Mechanical and Civil Engineering," Kluwer Academic Publishers, May 1999.

100. "Specifications and Tests for Piezoelectric Pressure and Sound-Pressure Transducers: ISA Standard S37.10," Instrument Society of America, 1982.

101. Y. Yuan and F.F. Ling, Vibrations of Elastic Plates: Linear and Nonlinear Dynamical Modeling of Sandwiches, Laminated Composites, and Piezoelectric Layers, Springer-Verlag New York, January 1995.

102. K. Choi. and F.-K. Chang, "Identification of Impact Force and Location Using Distributed Sensors," AIAA Journal, Vol. 34, No. 1, pp. 136-142, January 1996.

103. G. Hearn.and R.B. Testa, "Modal Analysis for Damage Detection in Structures," J. of Structural Engineering, Vol. 117, No. 10, pp. 3042-3063, October 1991.

104. J.-S. Lew, "Using Transfer Function Parameter Changes for Damage Detection of Structures," AIAA Journal, Vol. 33, No. 11, , pp. 2189-2193, November 1991.

105. R.Y. Liang, J. Hu, and F. Choy, "Quantitative NDE Technique for Assessing Damages in Beam Structures," Journal of Engineering Mechanics, Vol. 118, No. 7, 1468-1487, July 1992.

106. R.A. Manning, "Structural Damage Detection Using Active Members and Neural Networks," AIAA Journal, Vol. 32, No. 6, pp 1331-1333, 1994.

107. M.A. Moetakef, S.P. Joshi, and K.L. Lawrence, "Elastic Wave Generation by Piezo-ceramic Patches," AIAA Journal, Vol. 34, No. 10, pp. 2110-2117, October 1996.

108. N. Stubbs and J.-T. Kim, "Damage Localization in Structures Without Baseline Modal Parameters," AIAA Journal, Vol. 34, No. 8, , pp. 1645-1649, August 1996.

109. Z. Ounaies, "Piezoelectric Materials for Sensor and Actuator Applications at NASA LaRC," ICASE Research Quarterly. http://www.icase.edu/RQ/archive/v8n2/art1.html http://www.icase.edu

110. American Institute of Physics Website, http://www.aip.org 
111. Materials Engineering, University of Purdue's Website http://www.ecn.purdue.edu/Engr/

112. G. Kawiecki, "Feasibility of Applying Distributed Piezo-transducers to Structural Damage Detection," Journal of Intelligent Material Systems and Structures, Vol. 9, pp. 189-197, March 1998.

113. P.W. Smith, "Validation of Torsional - Bending Piezoelectric Actuator Concept," MS Thesis, The University of Tennessee, December 1993.

114. R.Kail and W. Mahr, "Piezoelectric Measuring Instruments and Their Applications," Translated from the article $<<$ Piezoelektrische Messgerate und ihre Anwendungen $>>$ edited in $<<$ Messen und Prufen $>>$, Vol. 20., 1984.

115. D. Shiozawa, S. Kubo, and T. Sakagami, "Passive Electric Potential CT Method Using Piezoeletric Material For Crack Identification," $4^{\text {th }}$ International Conference on Inverse Problems in Engineering, Rio de Janeiro, Brazil, 2002.

116. L.M. Brekhovskikh, O.A. Godlin, Acoustics of Layered Media I, Springer-Verlag, New York, 1990.

117. L.M. Brekhovskikh, Yu. P. Lysanov, Fundamentals of Ocean Acoustics, Springer-Verlag, New York, 1991.

118. B.R. Upadhyaya, J.W. Hines, X. Huang, B. Lu, S.R. Perillo, and R.L. Penha, OnLine Monitoring and Diagnostics of the Integrity of Nuclear Plant Steam Generators and Heat Exchangers, Annual Report: Phase-2, prepared for the DOENEER Program by the University of Tennessee, Knoxville, Report No. DE-FG0701ID14114/UTNE-04, June 2003.

119. F. Asano, S. Ikeda, M. Ogawa, H. Asoh, N. Kitawaki, "Combined Approach of Array Processing and Independent Component Analysis for Blind Seperation of Acoustic Signals," IEEE Transactions on Speech and Audio Processing, Vol. 11, No. 3, pp.204215, May 2003.

120. A. C. Tam, "Application of Photoacoustic Sensing Techniques," Reviews of Modern Physics, Vol. 58, No. 2, pp.381-431, April, 1986.

121. A.C. Raich, A. Cinar, "Multivariate Statistical Methods for Monitoring Continuous Processes: Assessment of Discrimination Power of Disturbance Models and Diagnosis of Multiple Disturbance," Chemometrics and Intelligent Laboratory System, No. 30, pp.37-48, 1995.

122. F. Ahmad, "A Simple Formula for the Lamb Modes in A Plate," J. Acoust. Soc. Am., Vol. 111, No. 5, pp. 1974-1975, May 2002. 


\section{Appendices}

\section{Appendix A: Cylindrical Coordinate Used in Tube Analysis}

The cylindrical coordinate system used in tubular structure analysis has the following relations with rectangular coordinate system:

$$
\begin{aligned}
& r=\sqrt{x^{2}+y^{2}}, \\
& \theta=\arctan (y / x), \\
& z=z . \\
& \hat{r}=\hat{x} \cos (\theta)+\hat{y} \sin (\theta), \\
& \hat{\theta}=\hat{z} \times \hat{r}=-\hat{x} \sin (\theta)+\hat{y} \cos (\theta) \\
& \hat{z}=\hat{z} . \\
& \text { where, } \\
& \hat{r}, \hat{\theta}, \hat{z}: \text { the unit vector in cylindrical coordinate system. }
\end{aligned}
$$

The variation of unit vectors with the coordinate can be derived as:

$$
\begin{aligned}
& \frac{\partial \hat{r}}{\partial r}=0 ; \quad \frac{\partial \hat{\theta}}{\partial r}=0 ; \quad \frac{\partial \hat{z}}{\partial r}=0 \\
& \frac{\partial \hat{r}}{\partial \theta}=-\hat{x} \sin (\theta)+\hat{y} \cos (\theta) ; \frac{\partial \hat{\theta}}{\partial \theta}=-\hat{x} \cos (\theta)-\hat{y} \sin (\theta)=-\hat{r} ; \frac{\partial \hat{z}}{\partial \theta}=0 \\
& \frac{\partial \hat{r}}{\partial z}=0 ; \quad \frac{\partial \hat{\theta}}{\partial z}=0 ; \quad \frac{\partial \hat{z}}{\partial z}=0 .
\end{aligned}
$$

The gradient in cylindrical system:

$$
\hat{\nabla}=\hat{r} \frac{\partial}{\partial r}+\frac{\hat{\theta}}{r} \frac{\partial}{\partial \theta}+\hat{z} \frac{\partial}{\partial z}
$$

The divergence of a vector $\mathrm{A}$ in cylindrical system:

$$
\begin{aligned}
& \hat{\nabla} \bullet \hat{A}=\frac{1}{r} \frac{\partial}{\partial r}\left(A_{r} r\right)+\frac{1}{r} \frac{\partial A_{\theta}}{\partial \theta}+\frac{\partial A_{z}}{\partial z} \\
& =\frac{\partial}{\partial r} A_{r}+\frac{A_{r}}{r}+\frac{1}{r} \frac{\partial A_{\theta}}{\partial \theta}+\frac{\partial A_{z}}{\partial z} .
\end{aligned}
$$

Then the Laplacian operator can be derived: 


$$
\Delta=\nabla^{2}=\frac{\partial^{2}}{\partial r^{2}}+\frac{1}{r} \frac{\partial}{\partial r}+\frac{1}{r^{2}} \frac{\partial}{\partial \theta}+\frac{\partial^{2}}{\partial z^{2}}
$$

The curl of a vector $\mathrm{A}$ in a cylindrical system is:

$\hat{\nabla} \times \hat{A}=\hat{r}\left(\frac{1}{r} \frac{\partial A_{r}}{\partial \theta}-\frac{\partial A_{\theta}}{\partial z}\right)+\hat{\theta}\left(\frac{\partial A_{r}}{\partial z}-\frac{\partial A_{z}}{\partial r}\right)+\hat{z}\left(\frac{1}{r} \frac{\partial}{\partial r}\left(A_{\theta} r\right)-\frac{1}{r} \frac{\partial A_{r}}{\partial \theta}\right)$.

The properties described here have been utilized in deriving the Lamb wave equations for brass tubes elaborated in chapter 6 . 


\section{Appendix B: More moving window results for brass tubes in air}

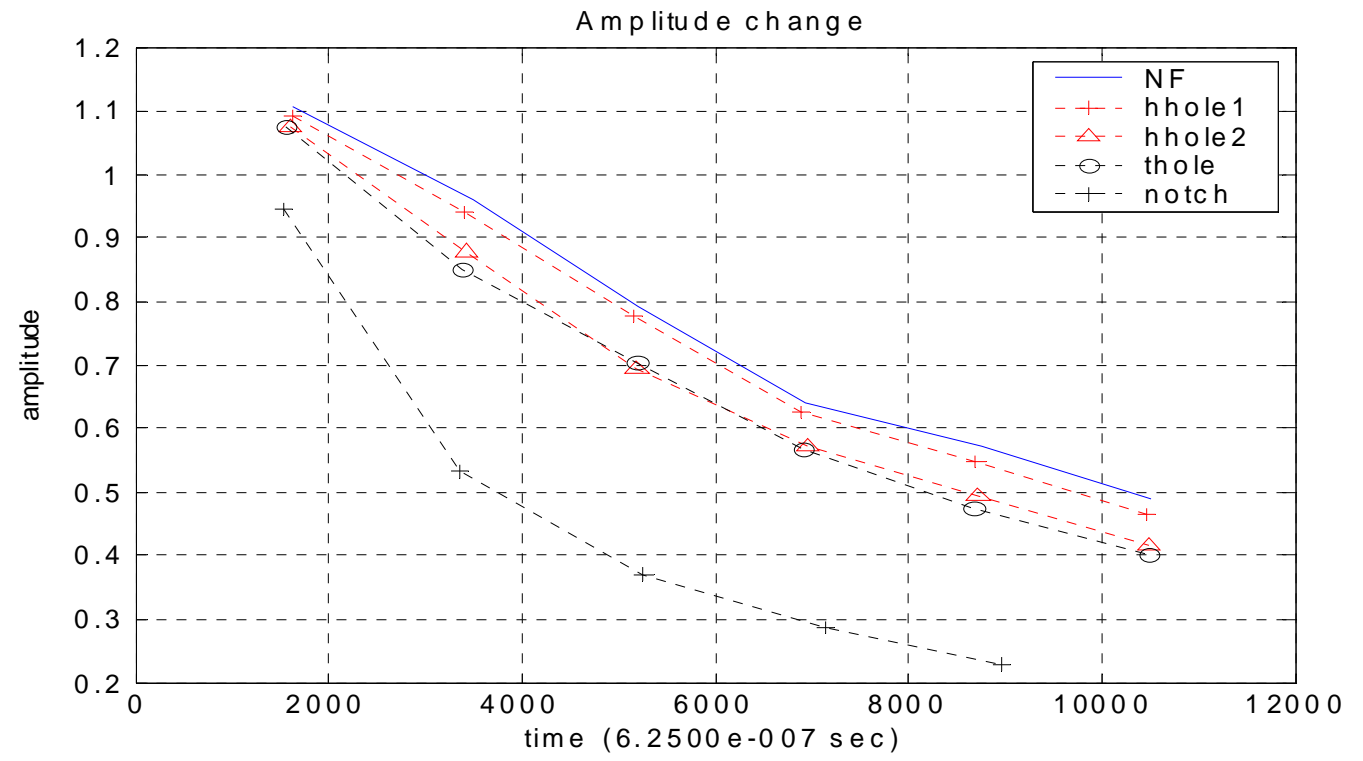

Figure A.1. Amplitude change of local peaks of anti-symmetric mode signals, propagating from left to right, input frequency $14 \mathrm{kHz}$.

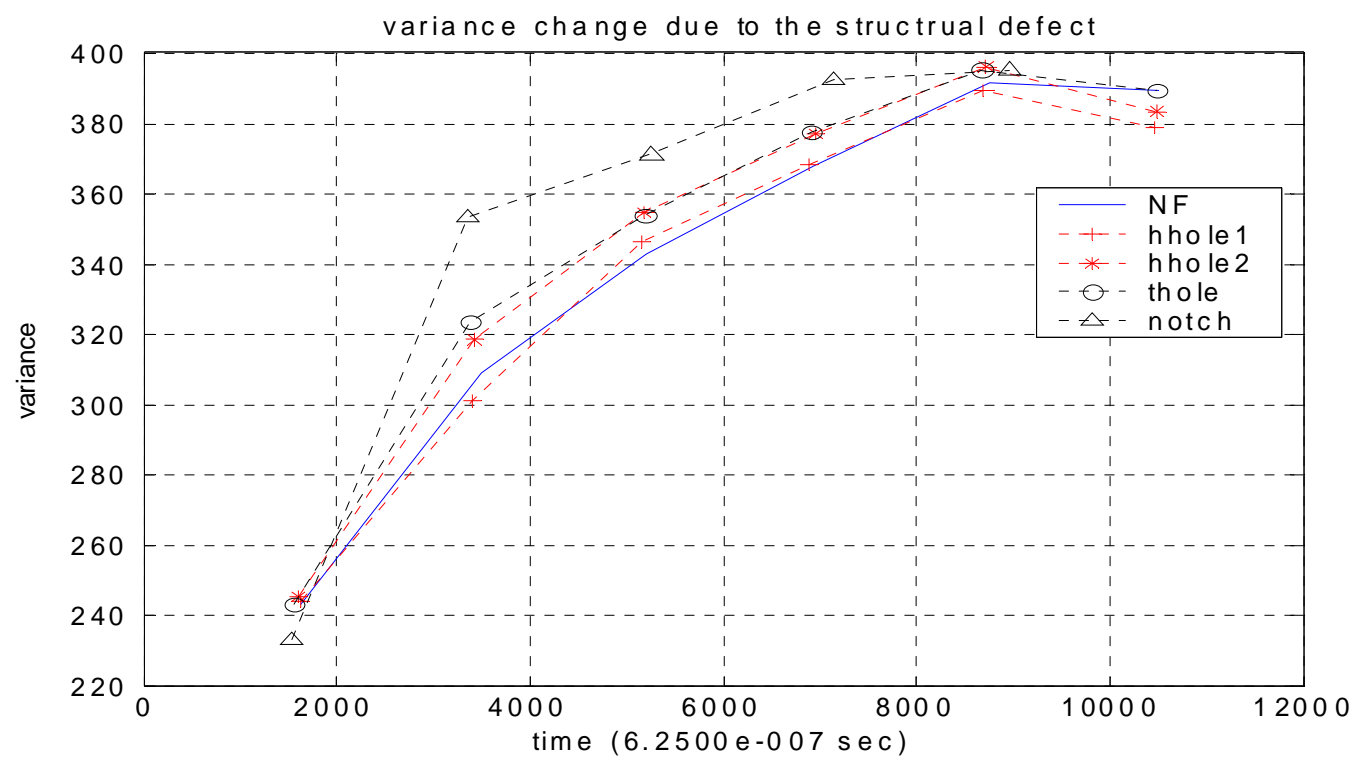

Figure A.2. The change in variance of local peaks of anti-symmetric mode signals, propagating from left to right, with $14 \mathrm{kHz}$ input frequency. 


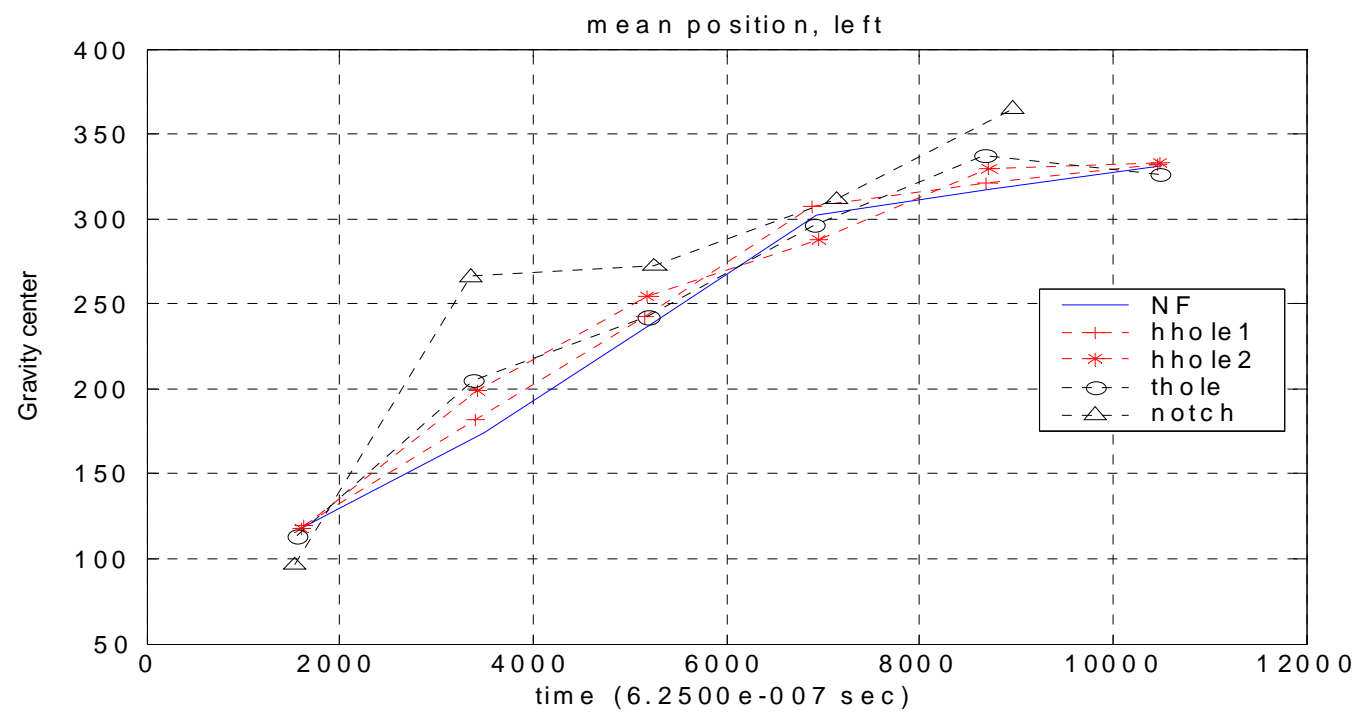

Figure A.3. The change in left part weight center of local peaks of anti-symmetric mode signals, propagating from left to right, input frequency $14 \mathrm{kHz}$.

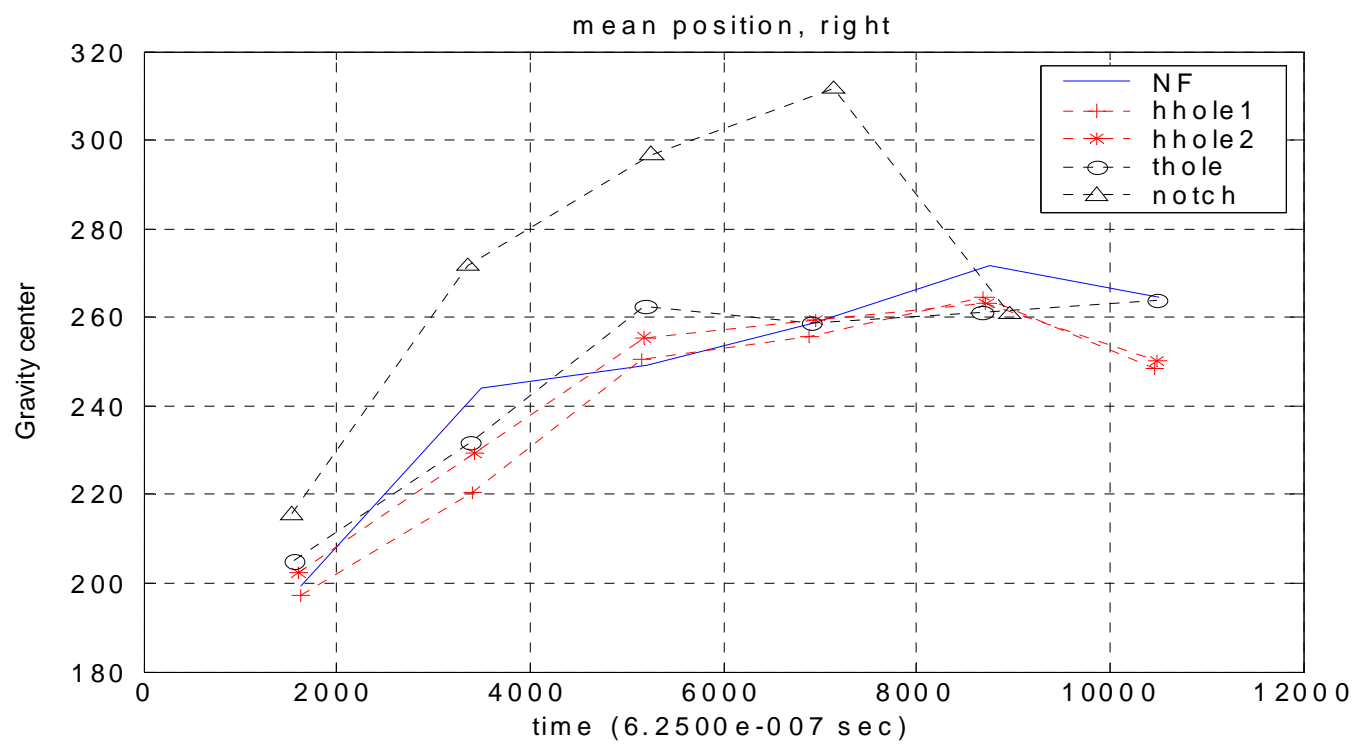

Figure A.4. The change in right part weight center of local peaks of anti-symmetric mode signals, propagating from left to right, input frequency $14 \mathrm{kHz}$. 


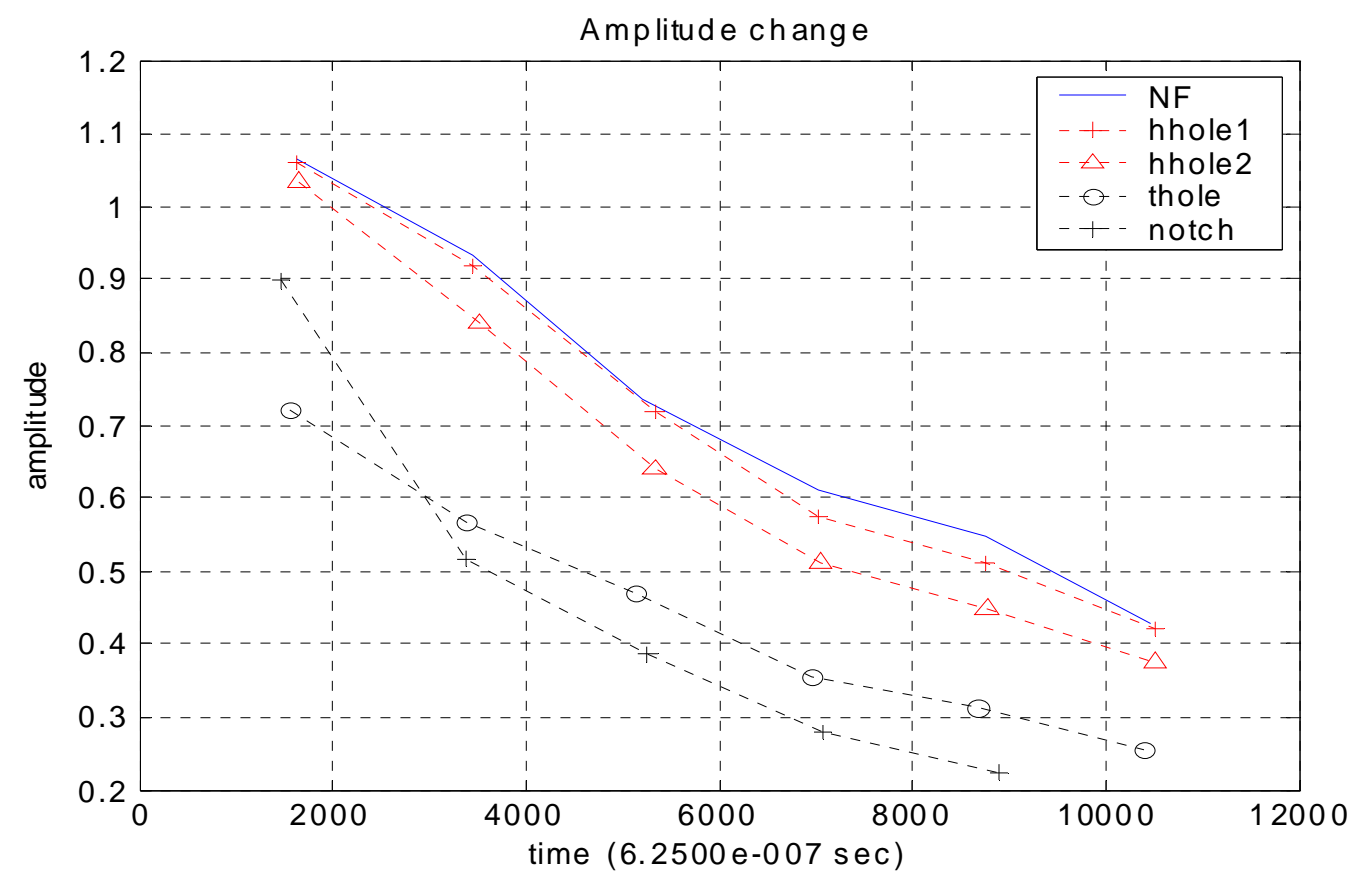

Figure A.5. Amplitude change in local peaks of anti-symmetric mode signals, propagating from left to right, input frequency $13 \mathrm{kHz}$.

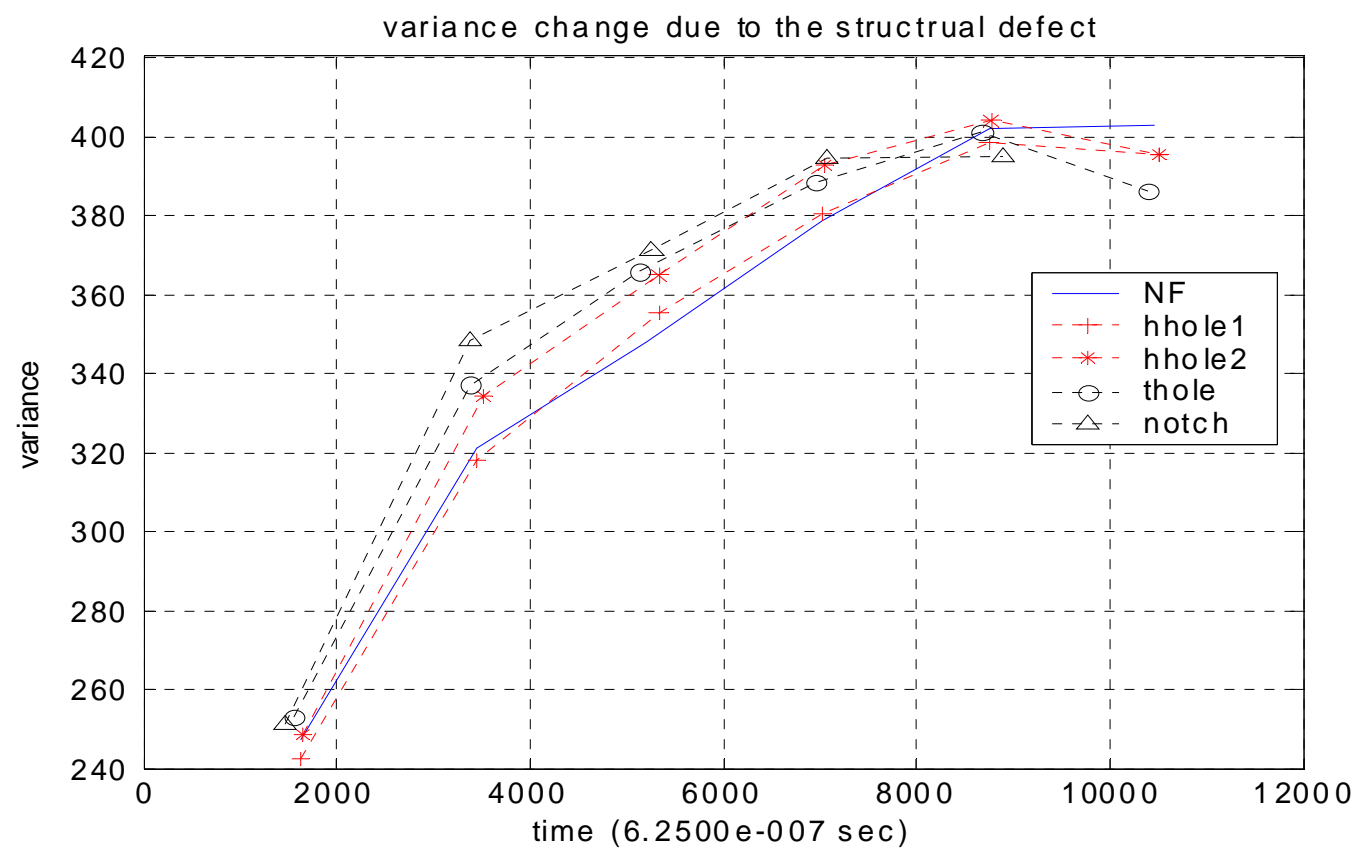

Figure A.6. The change in variance of local peaks of anti-symmetric mode signals, propagating from left to right, input frequency $13 \mathrm{kHz}$. 


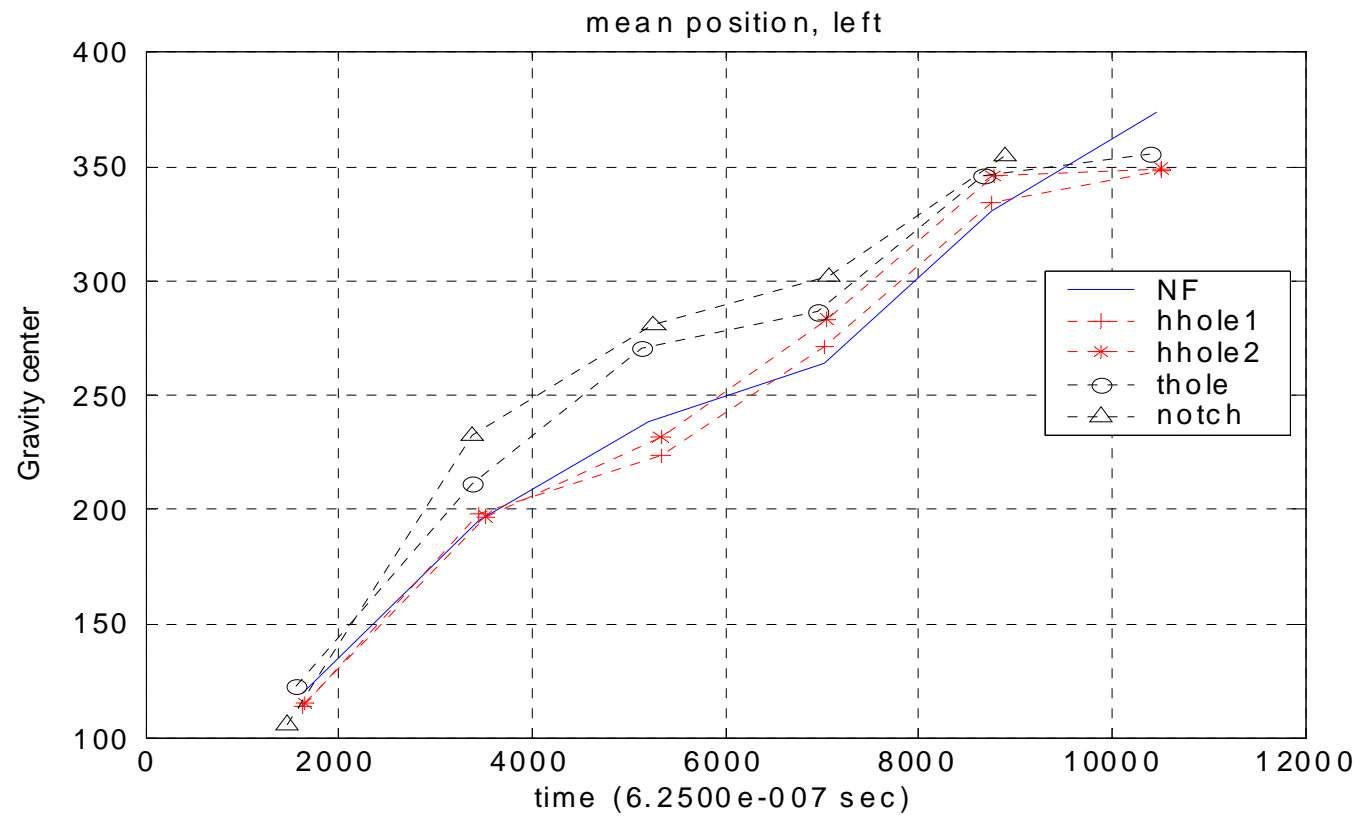

Figure A.7. The change in left part weight center of local peaks of anti-symmetric mode signals, propagating from left to right, input frequency $13 \mathrm{kHz}$.

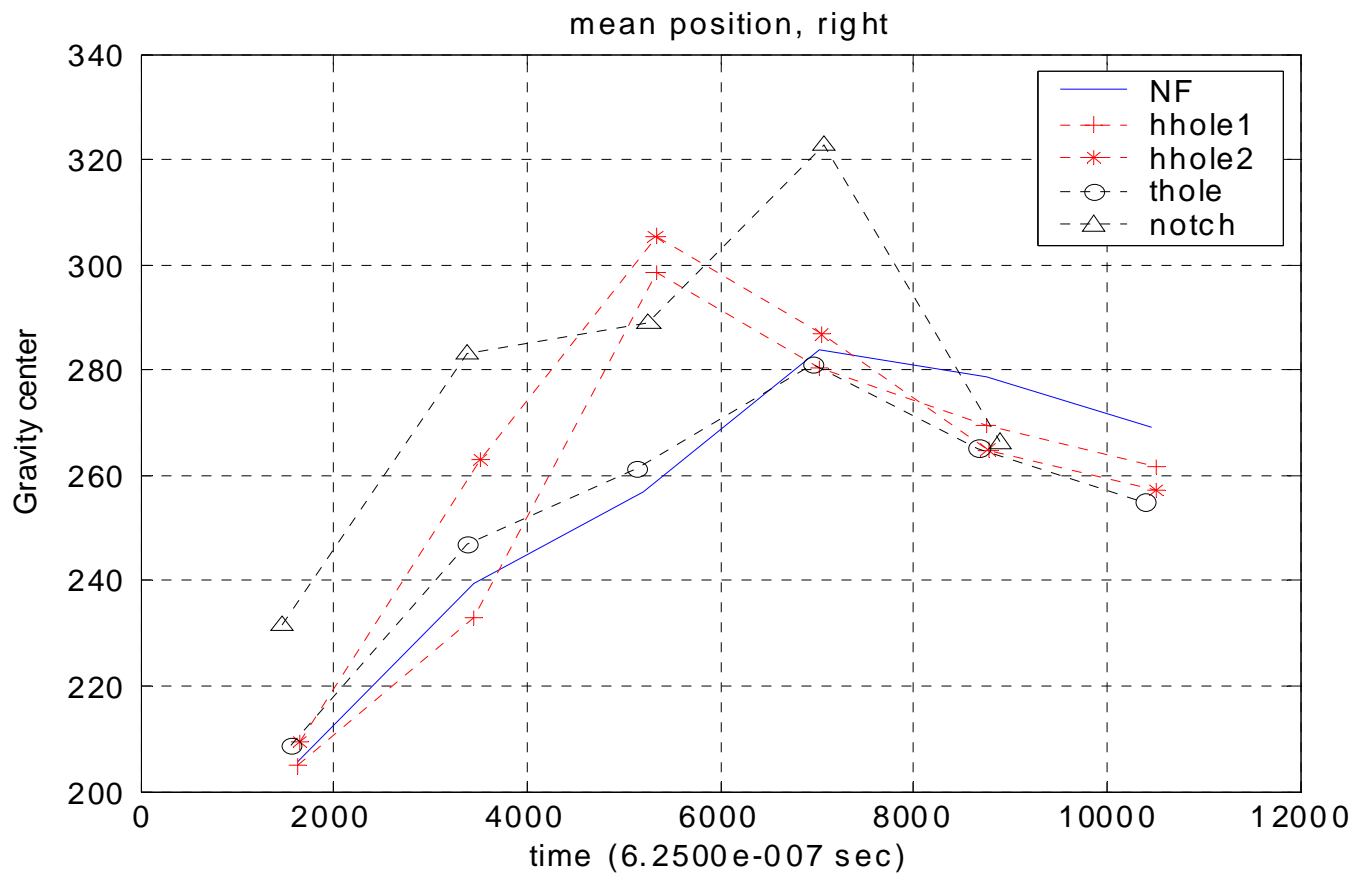

Figure A.8. The change in right part weight center of local peaks of anti-symmetric mode signals, propagating from left to right, input frequency $13 \mathrm{kHz}$. 


\section{Appendix C: MATLAB Code Lamb wave Numerical Solution}

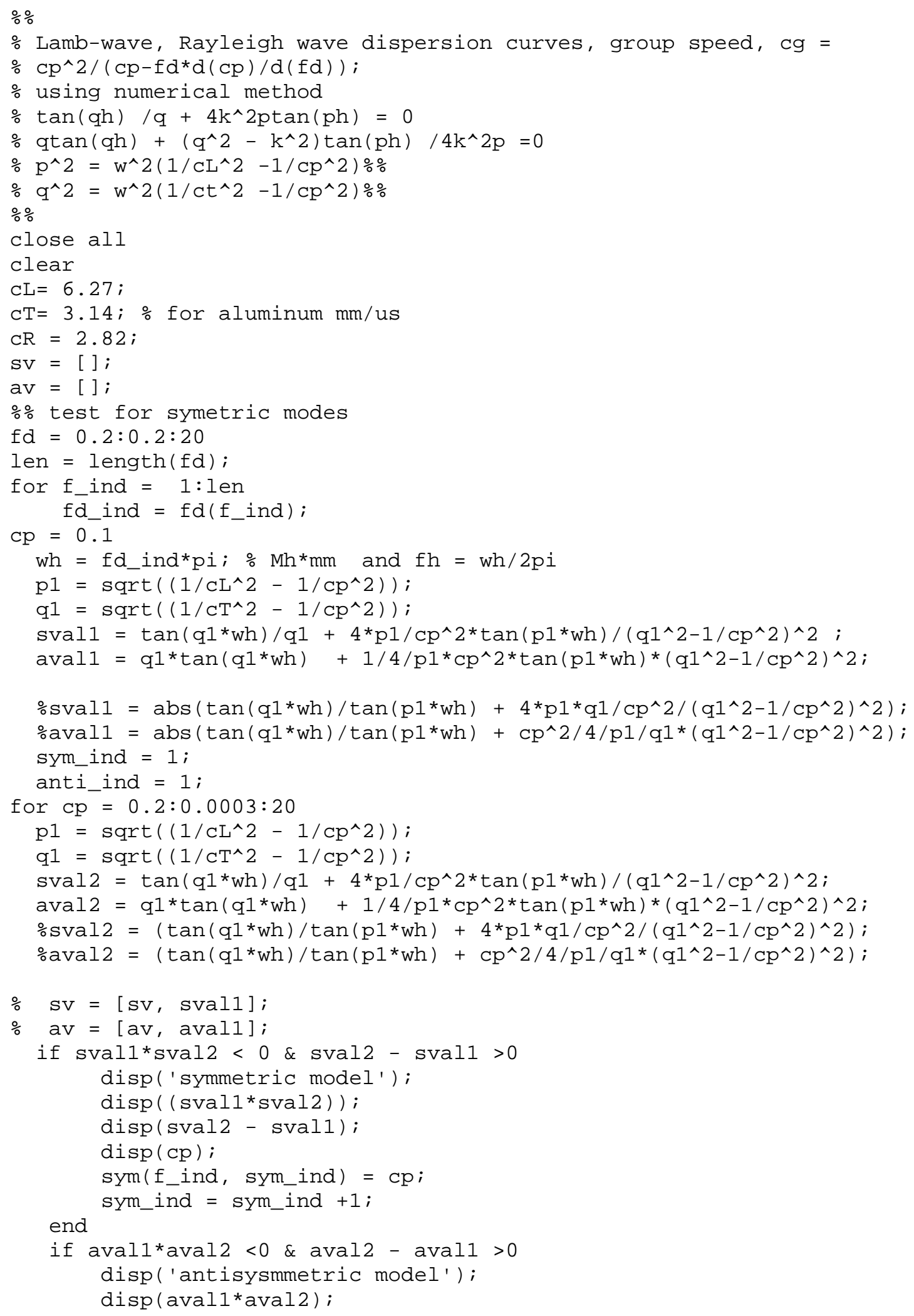




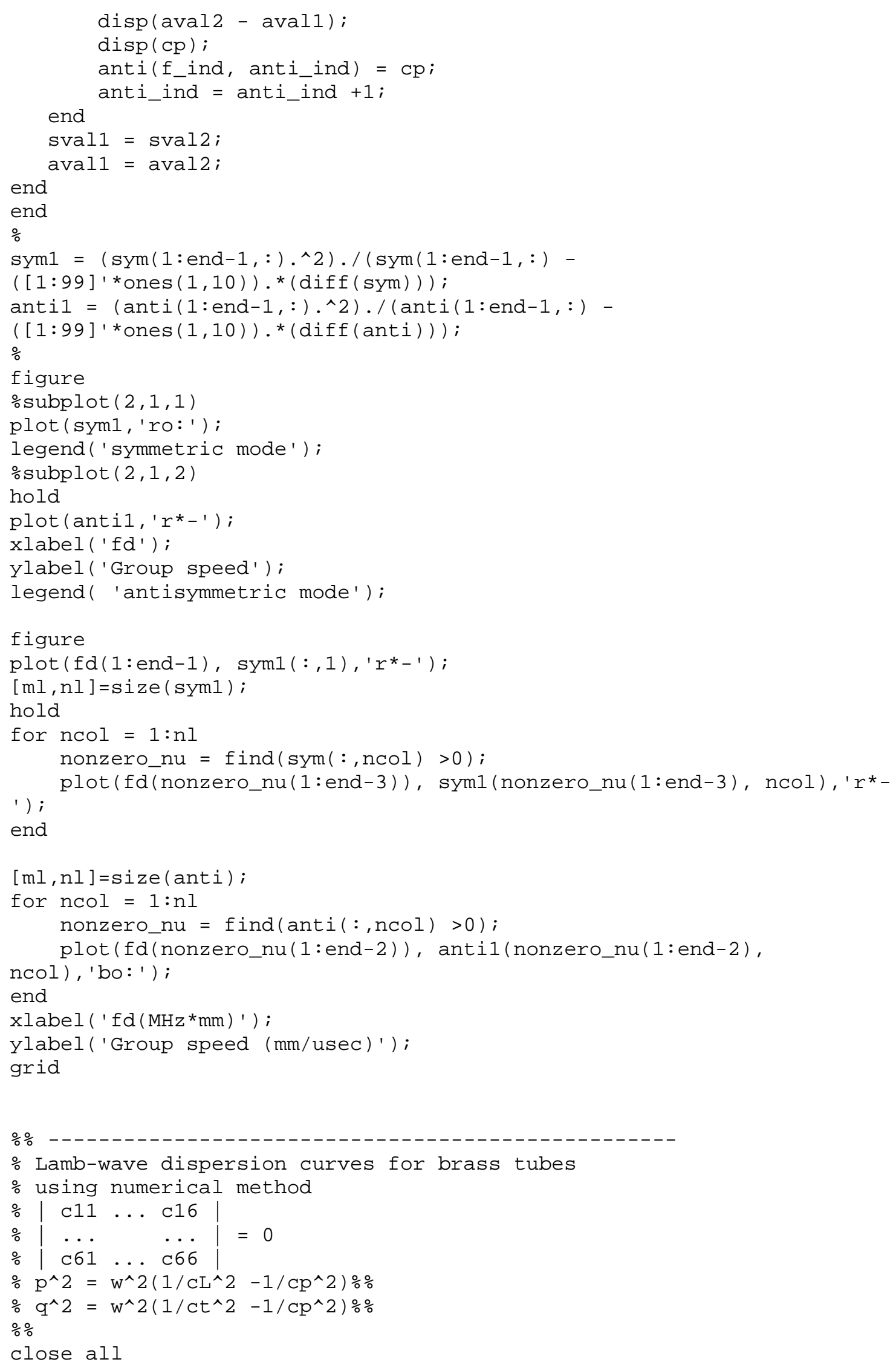




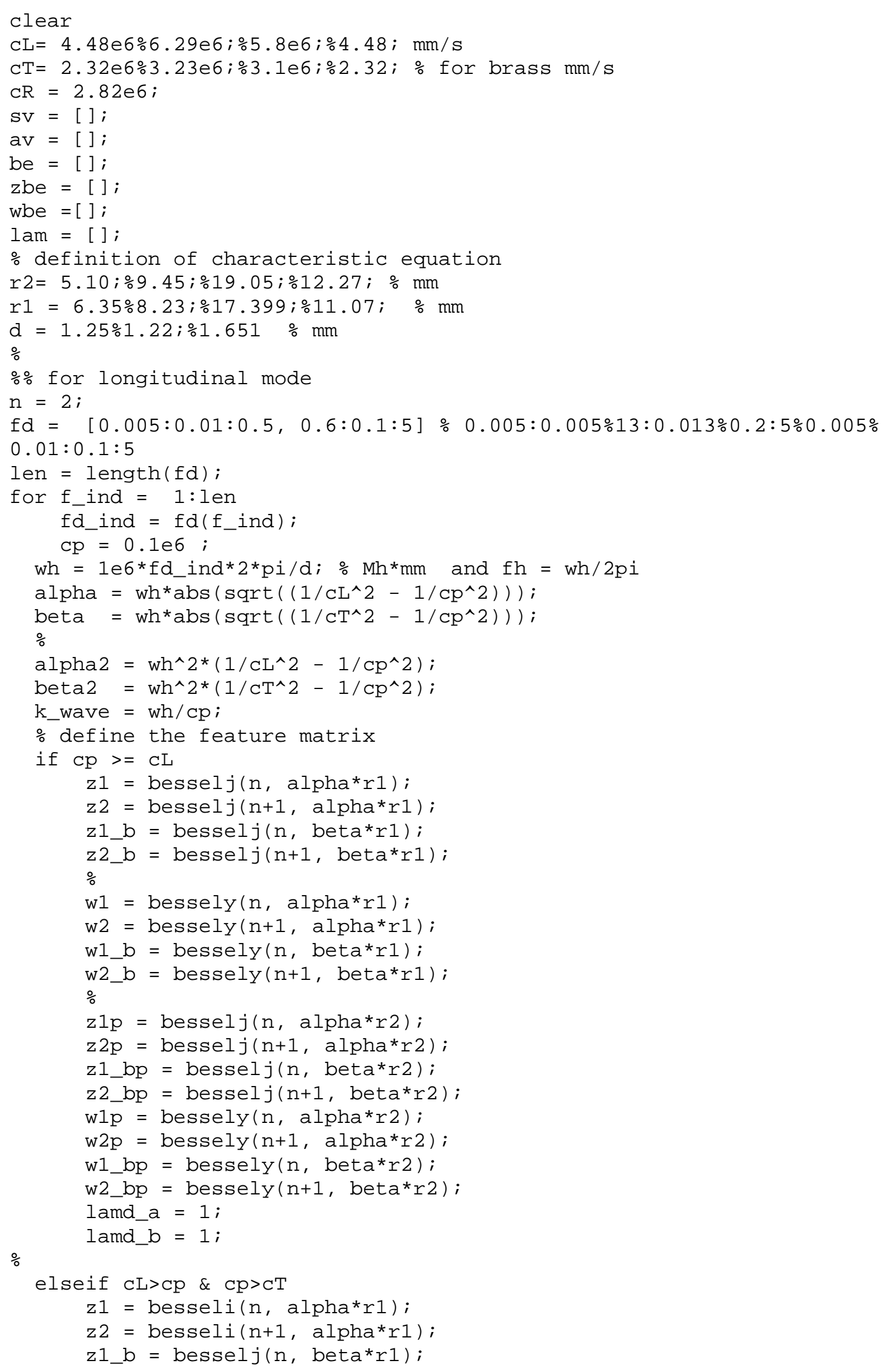




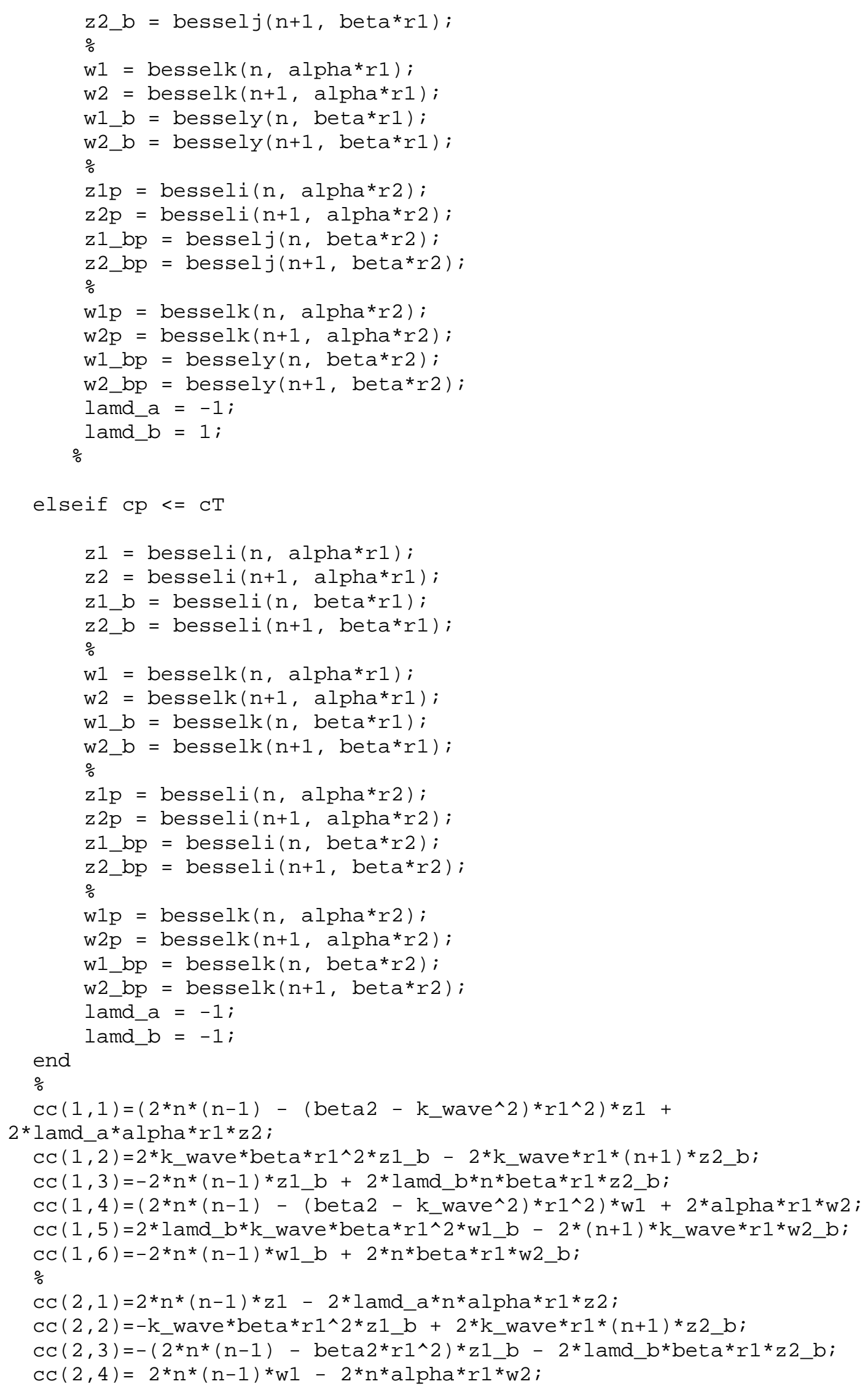




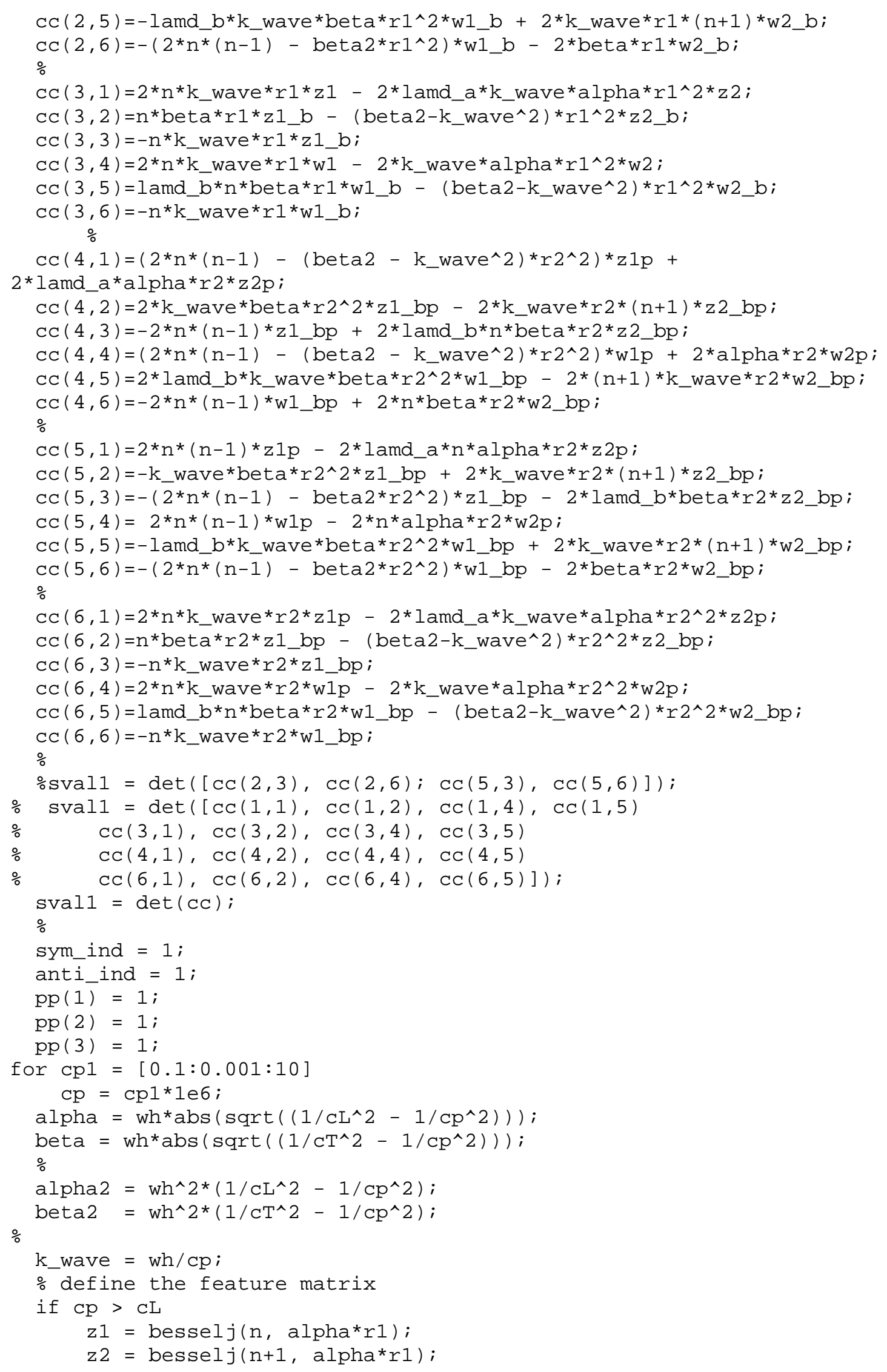




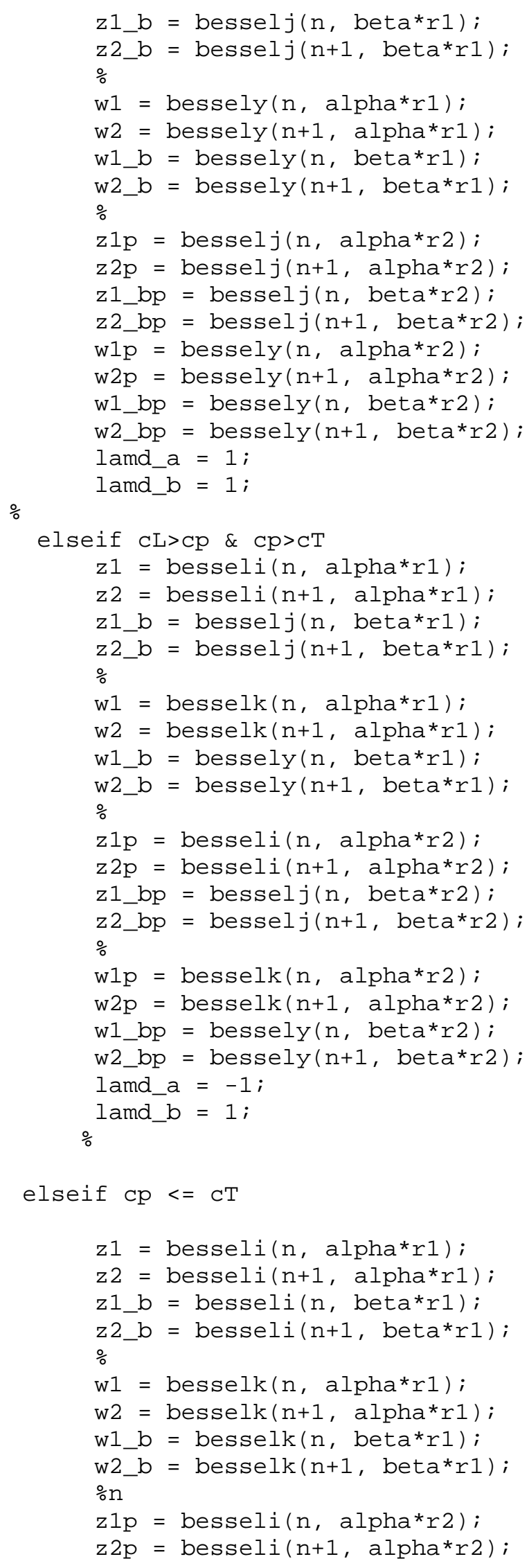




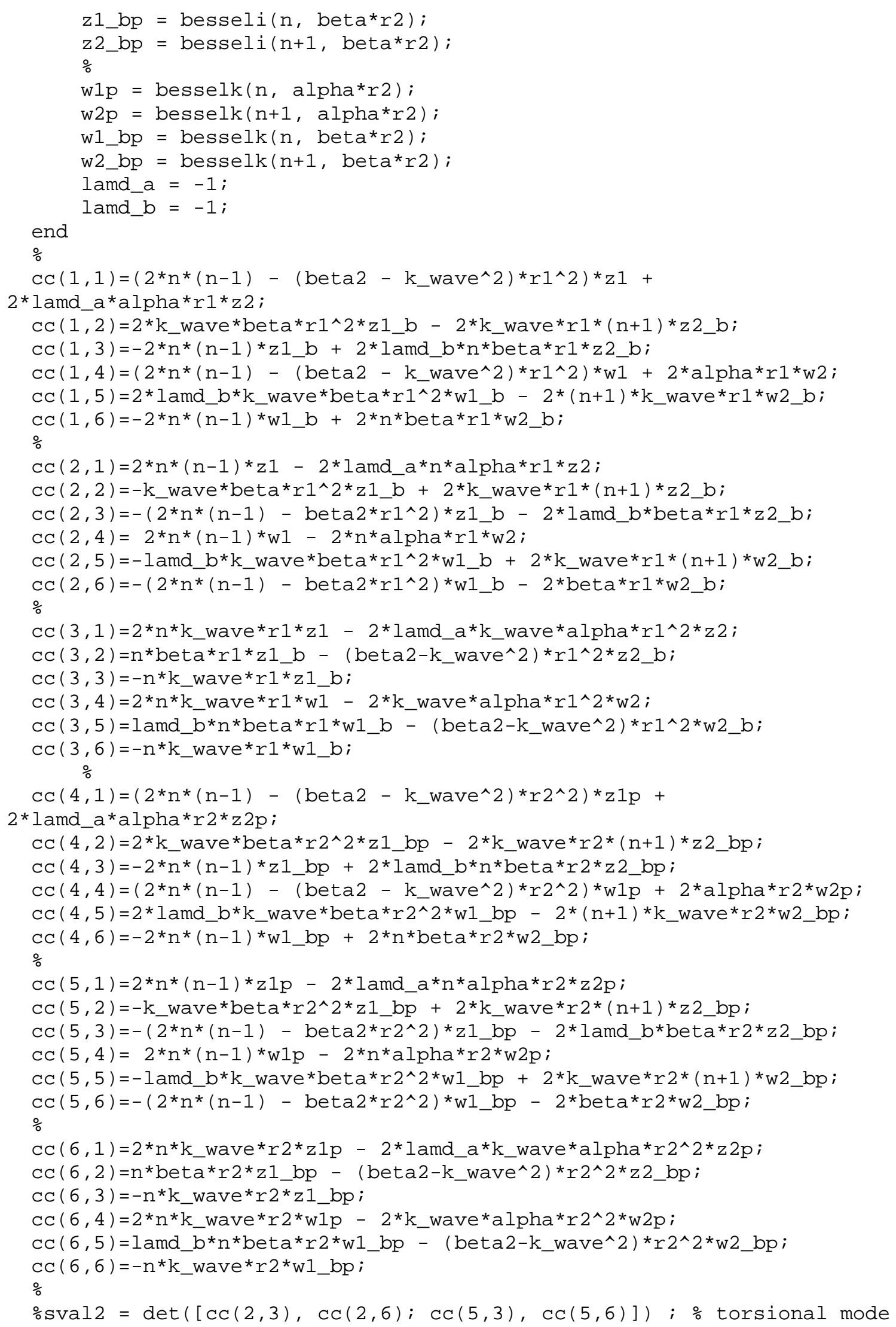




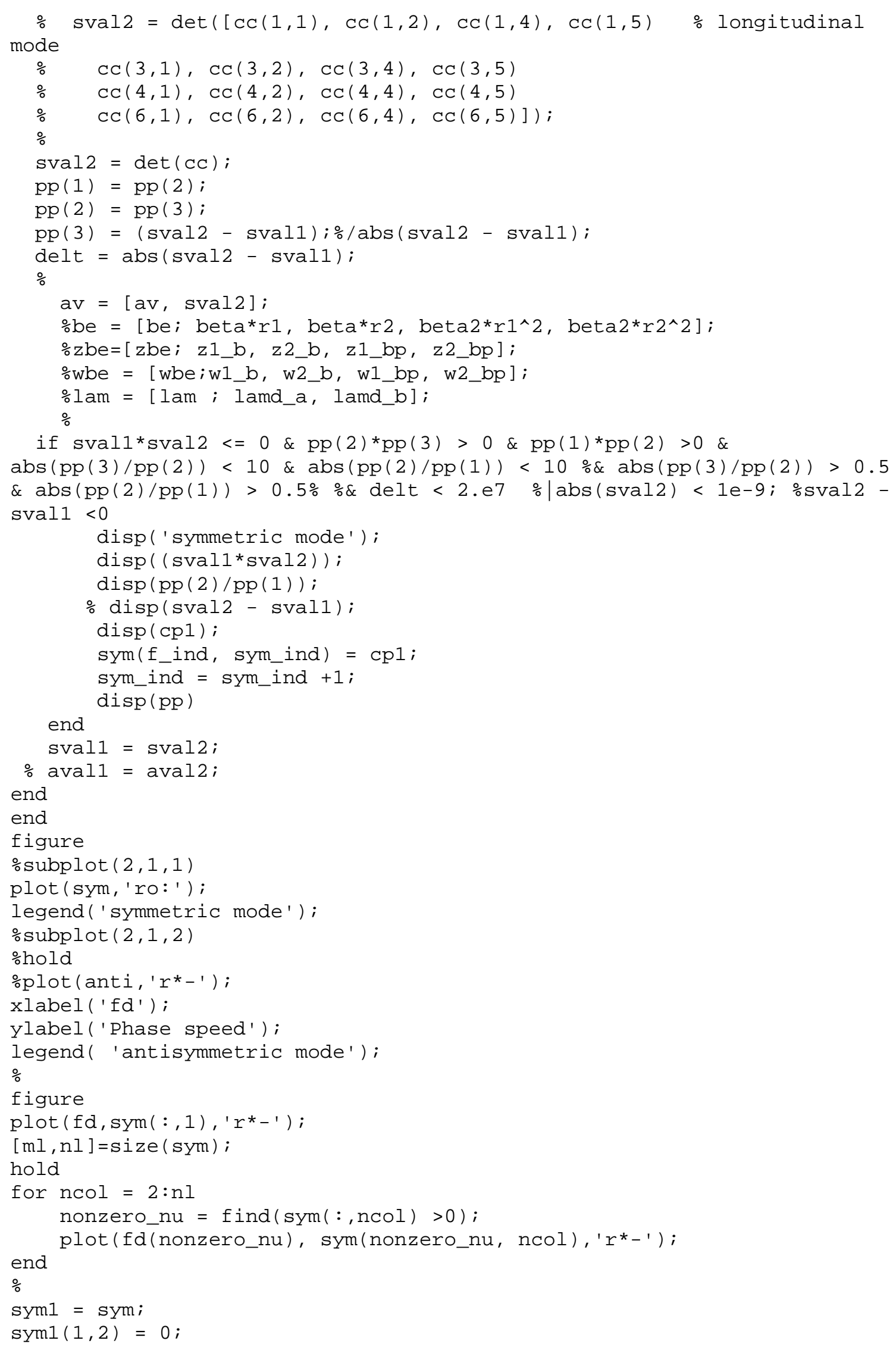




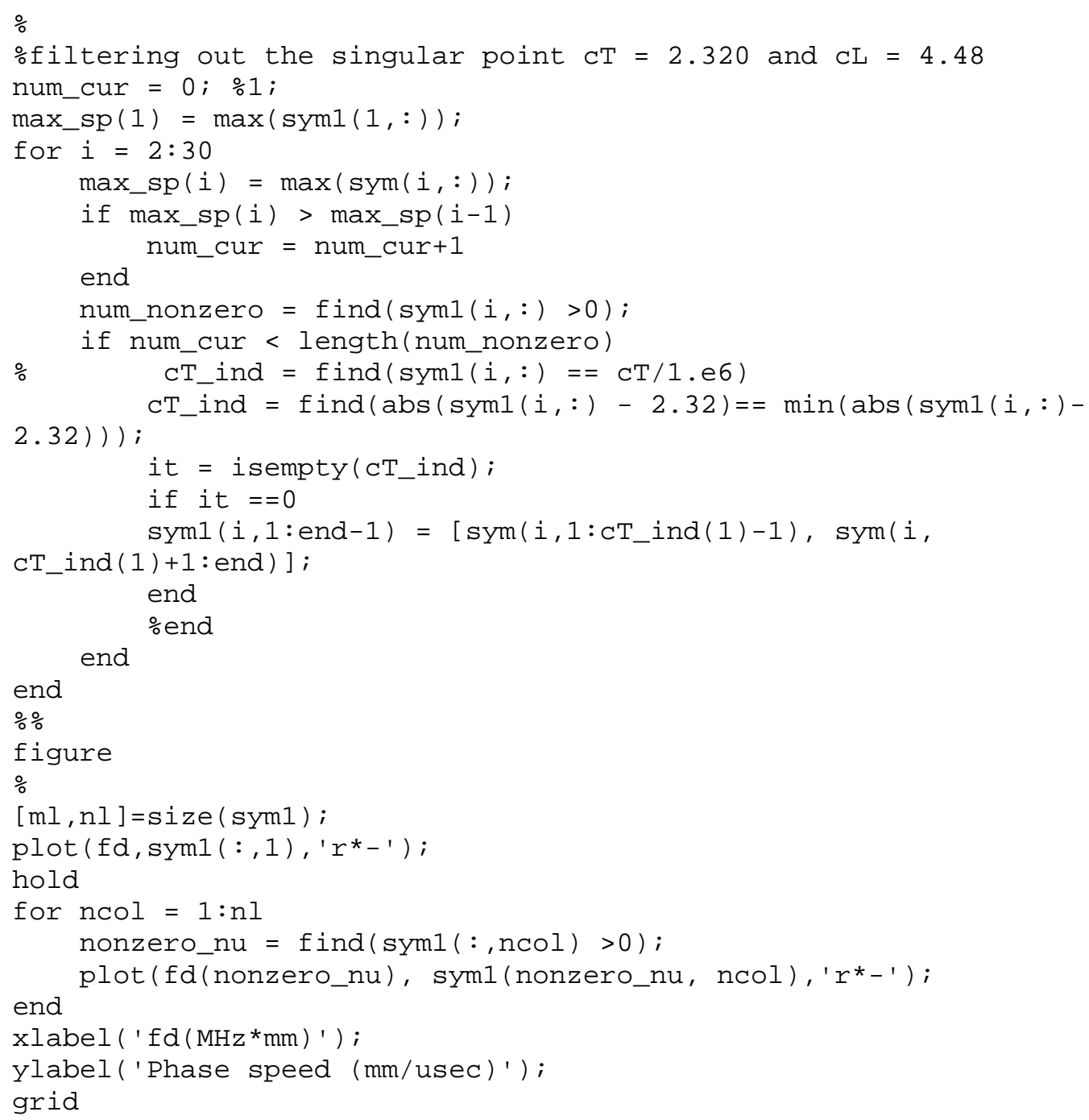




\section{Appendix D: MATLAB Code for HHT}

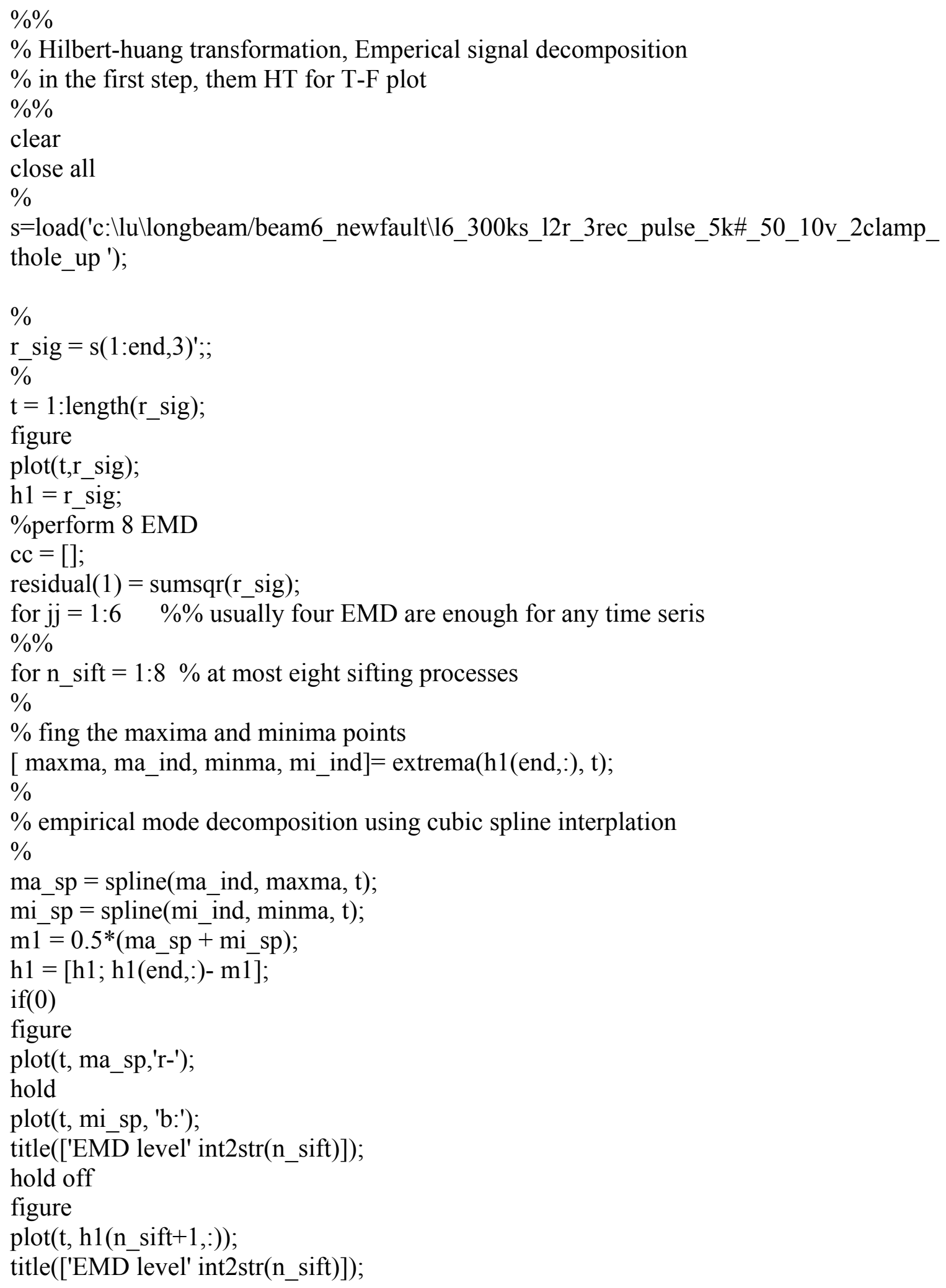




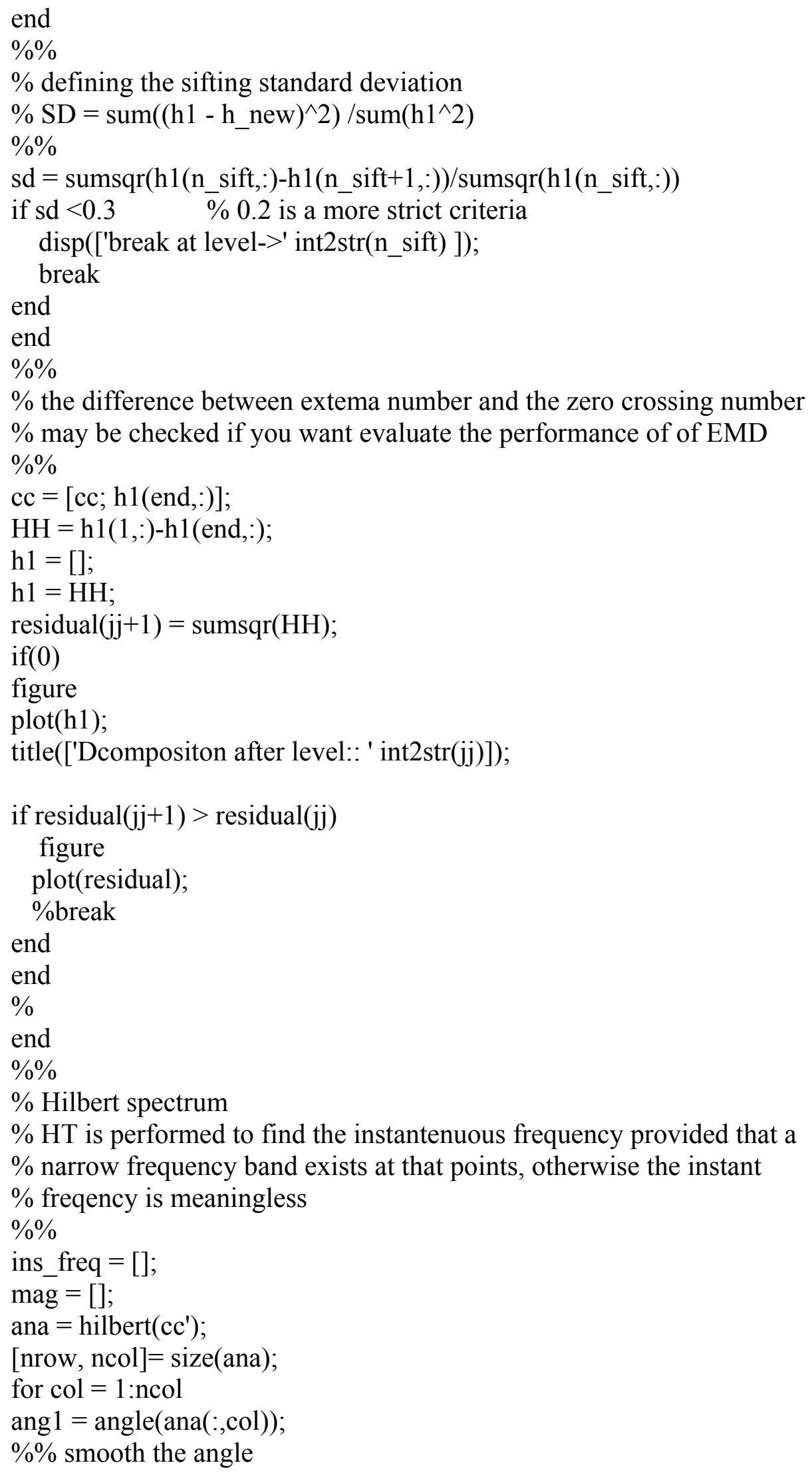




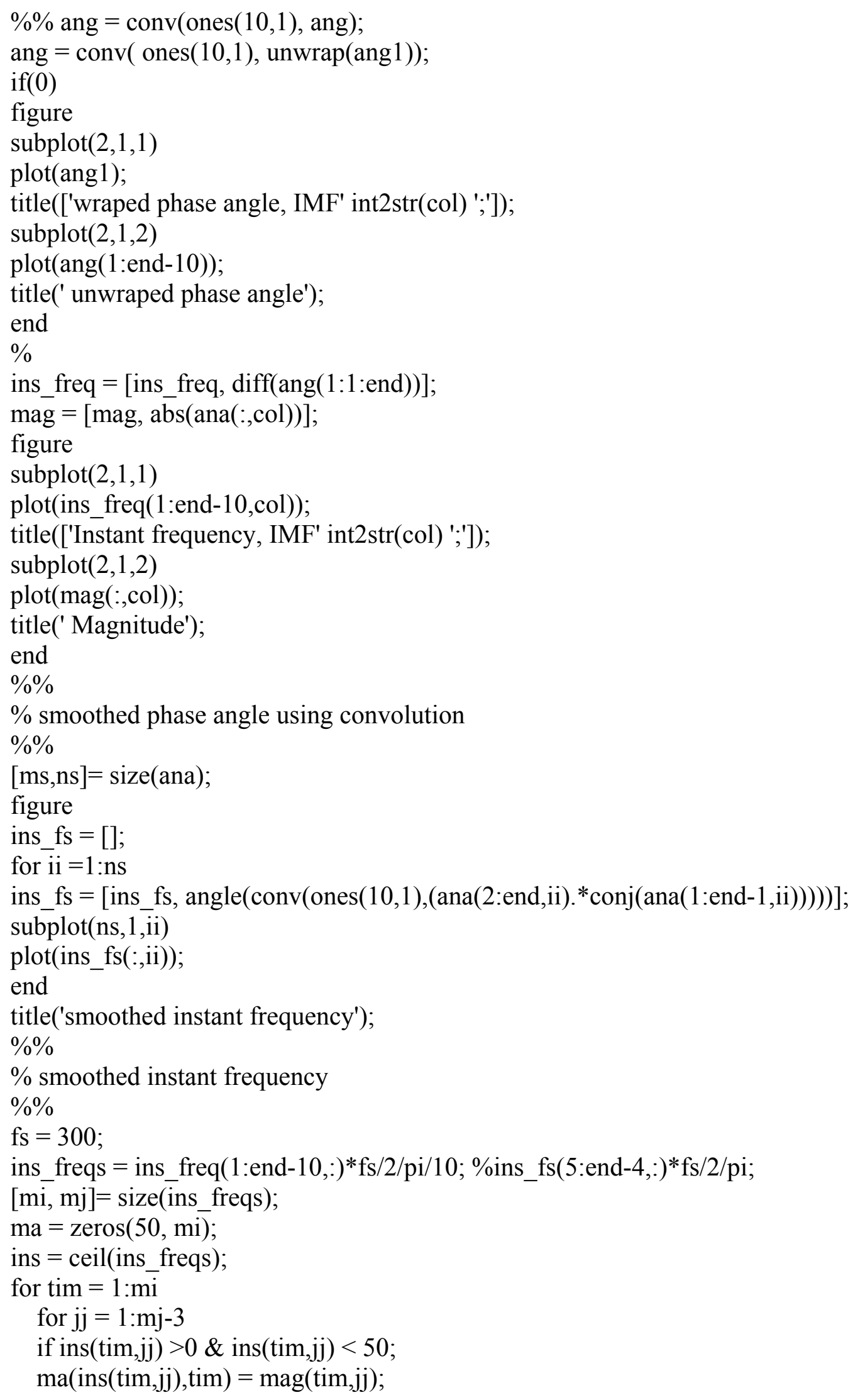




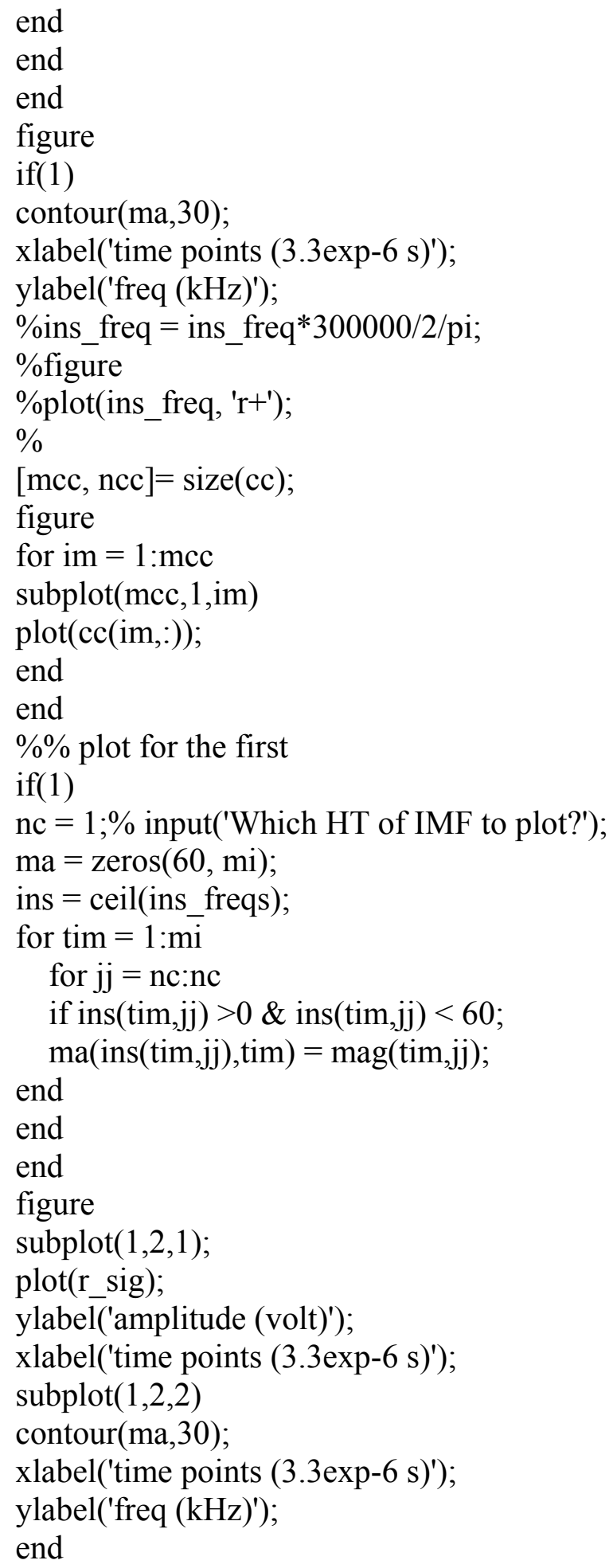




\section{Appendix E: MATLAB Code for Moving Window algorithm.}

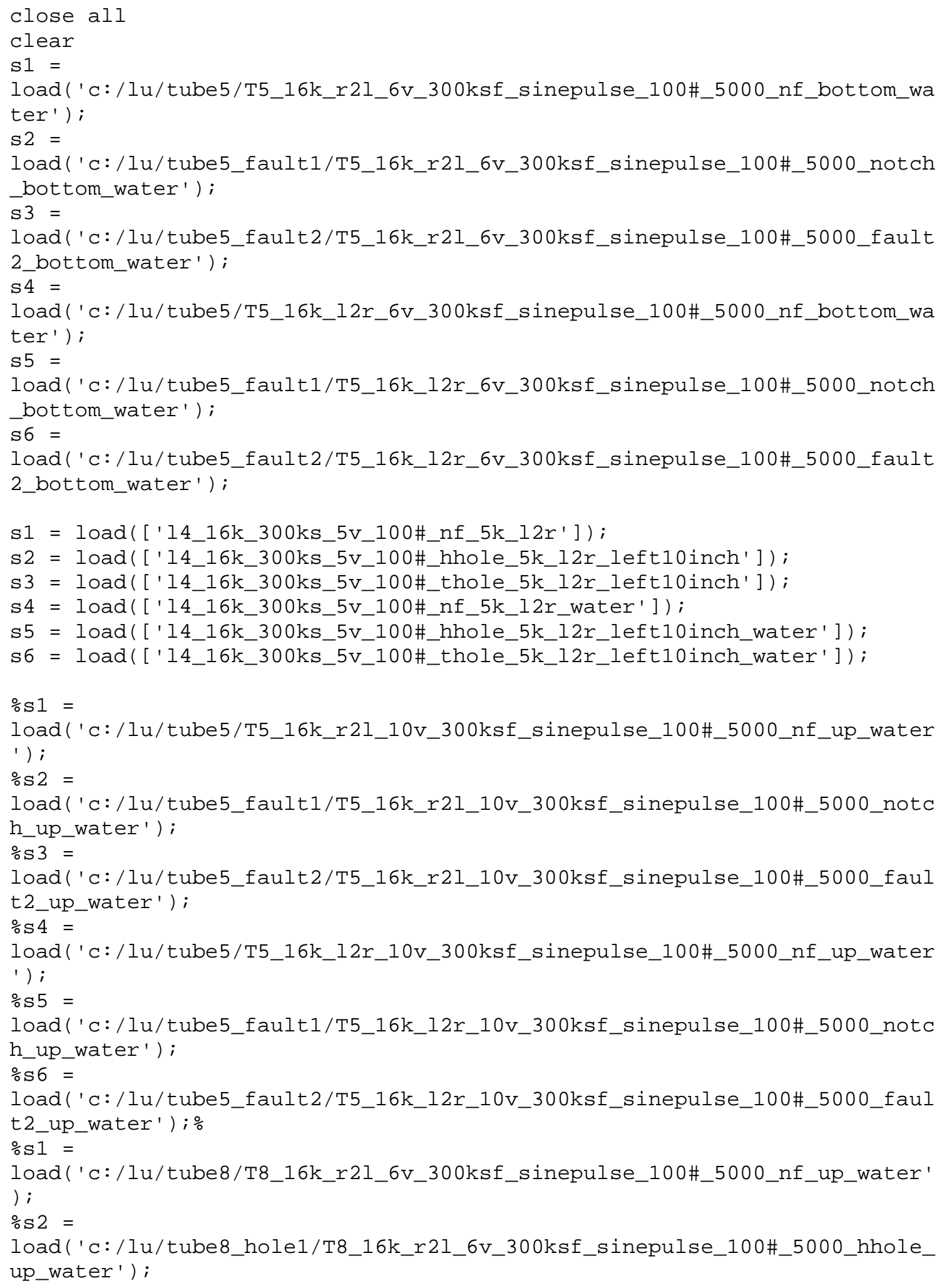




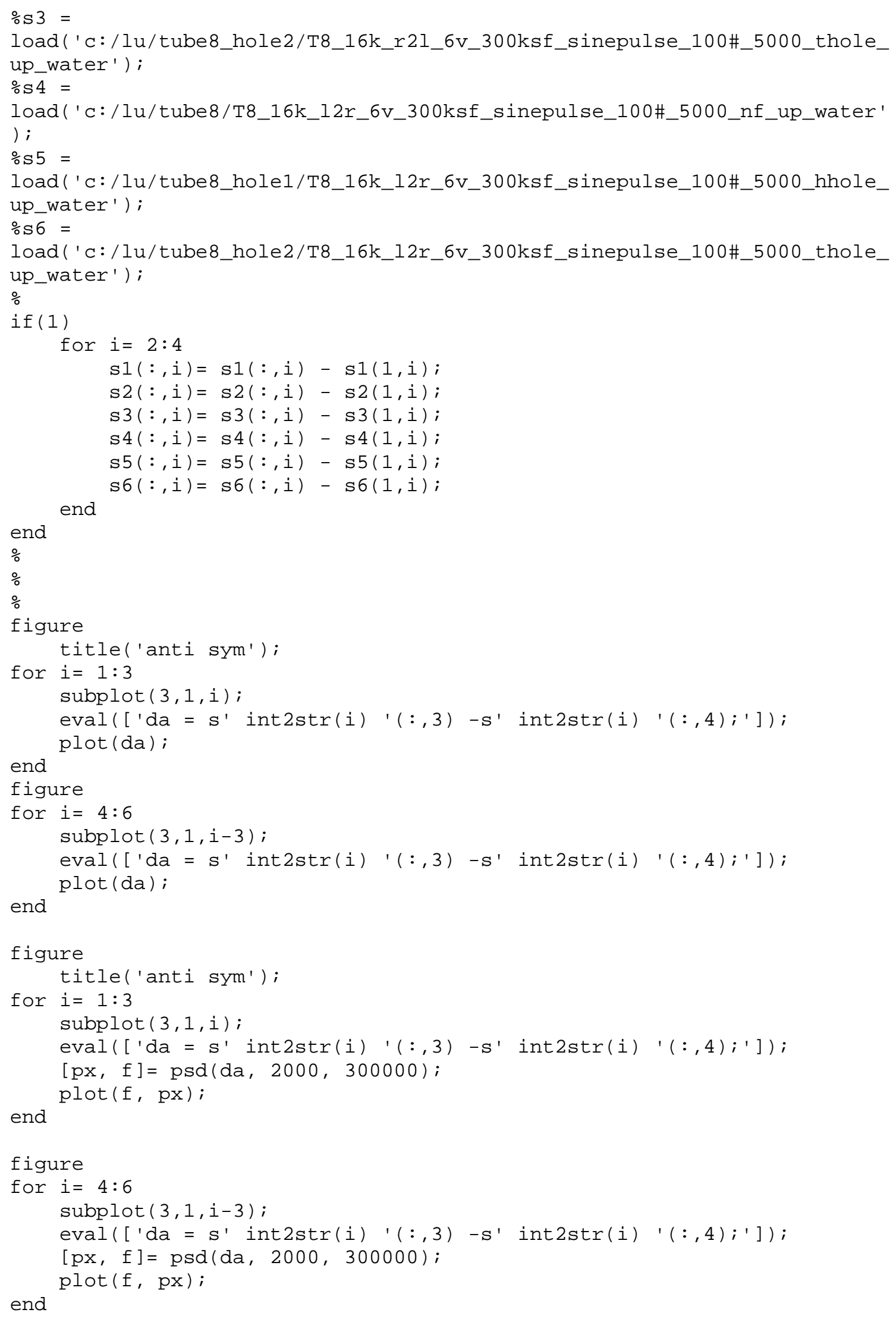




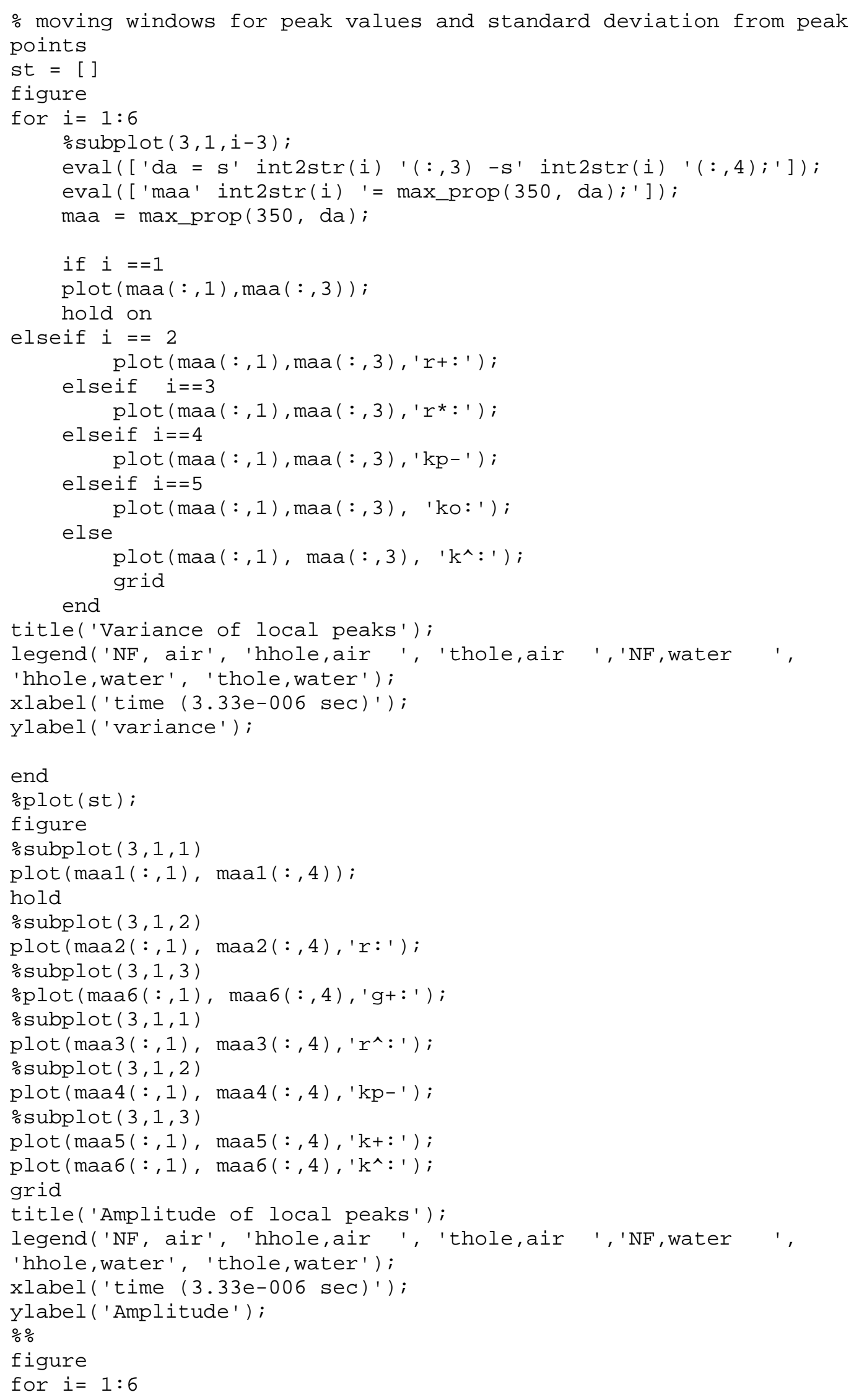




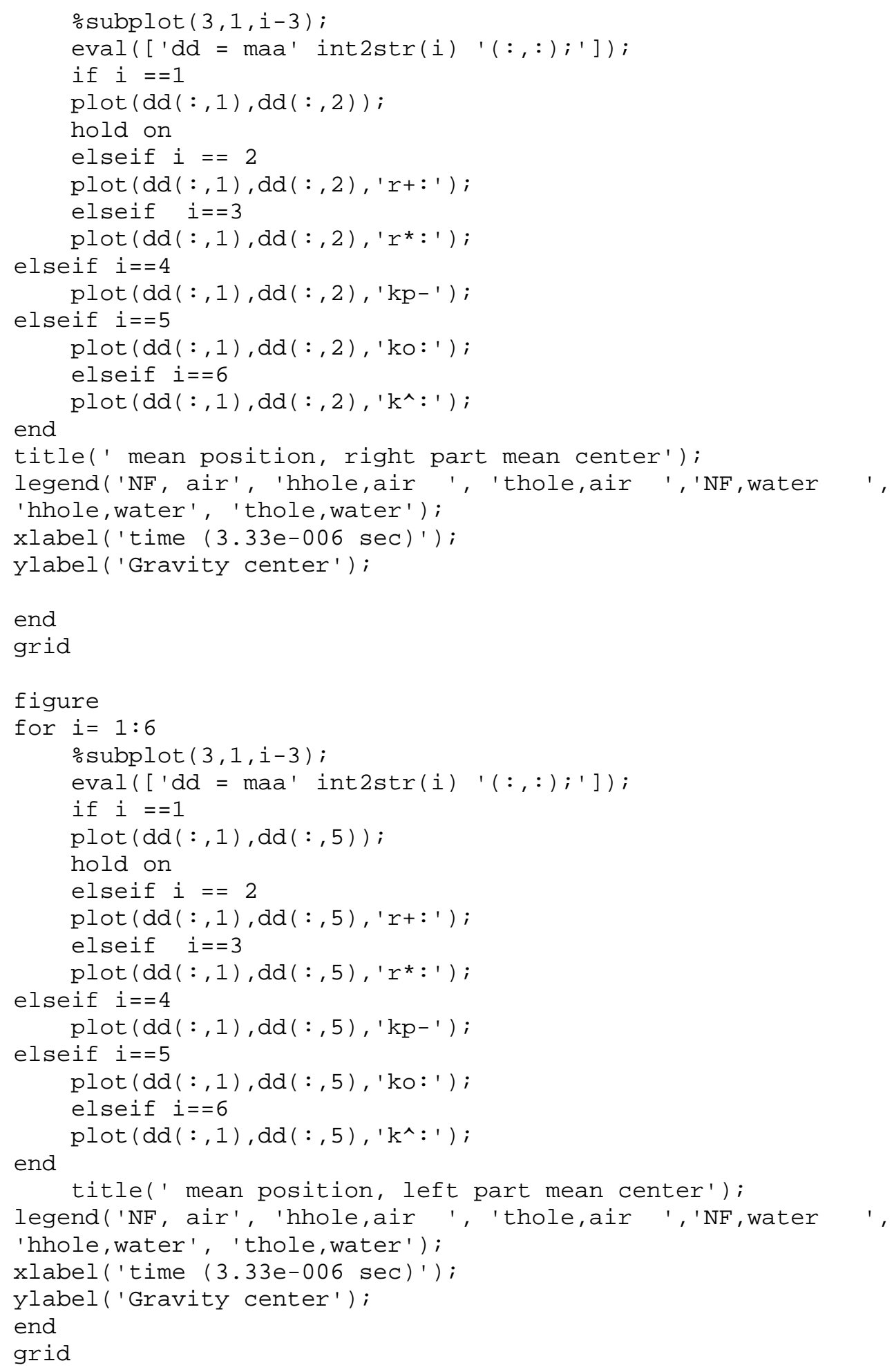




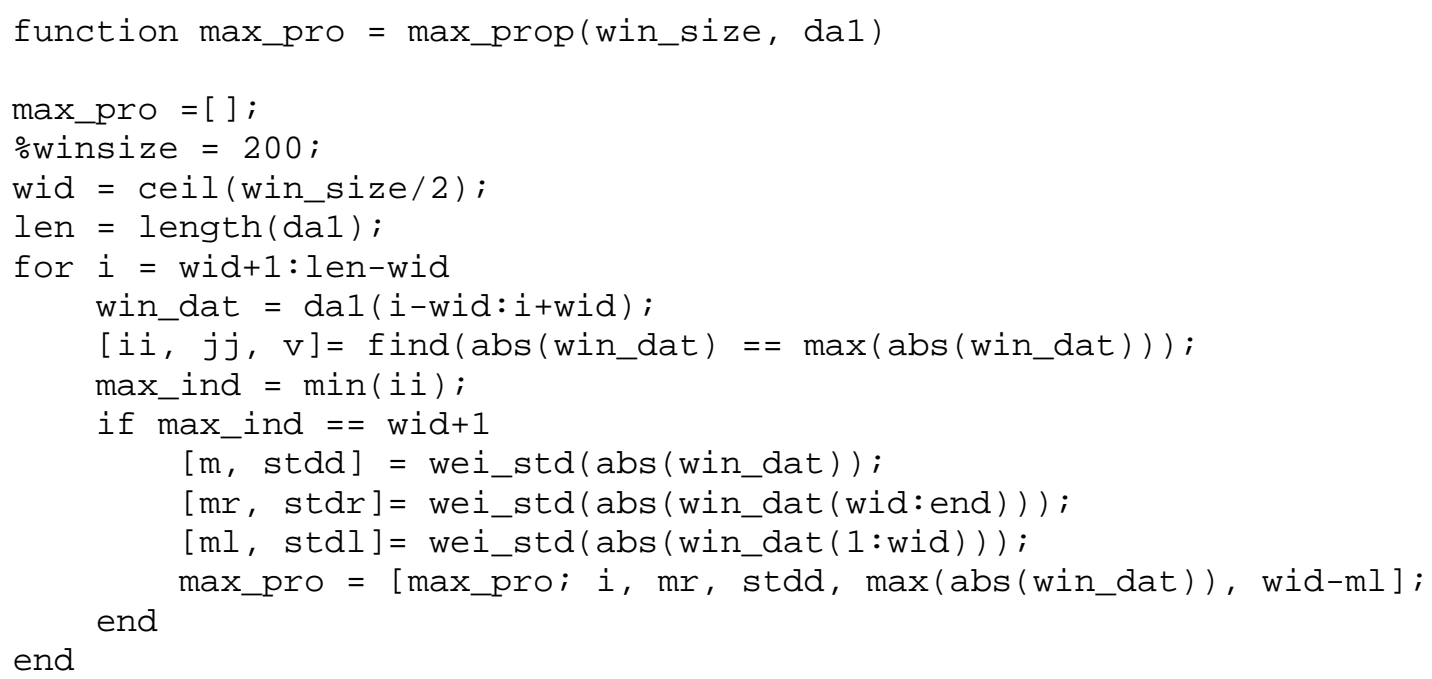


Appendix F: LabVIEW Interface for Lamb Wave Experiments 

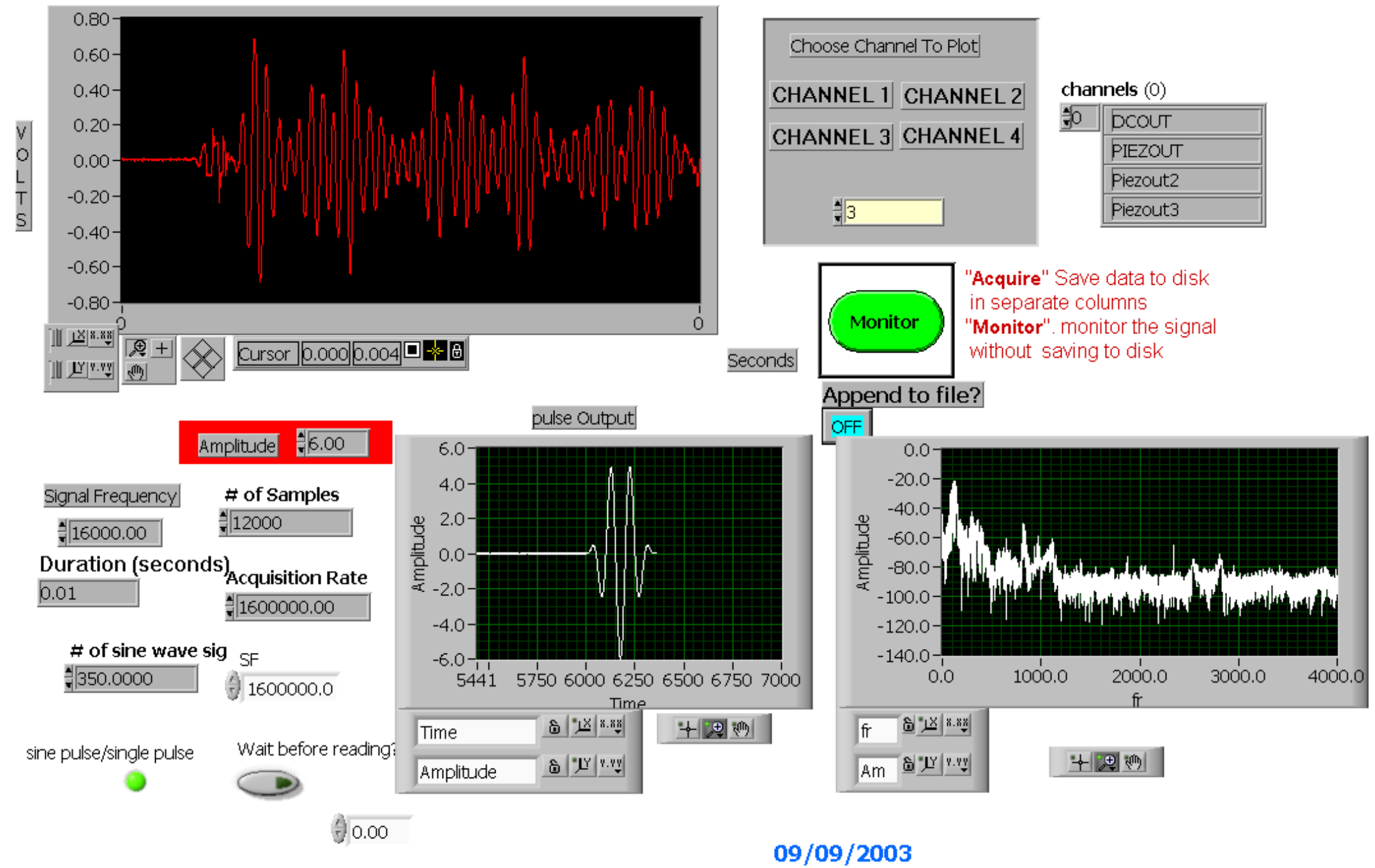